\title{
Combustión catalítica
}

de compuestos

orgánicos volátiles

utilizando sistemas

autotérmicos

con intercambio

de calor regenerativo

\section{Carlos Daniel Luzi}

Director: Dr. Guillermo F. Barreto Co-director: Ing. Osvaldo M. Martínez

Jurado:
Dr. Rodolfo Brandi
Dr. Eduardo López
Dr. Rodolfo Mascheroni 

A toda mi familia, especialmente a mi mamá y mi papá 

COMBUSTIÓN CATALÍTICA DE VOCS

UTILIZANDO SISTEMAS AUTOTÉRMICOS CON

INTERCAMBIO DE CALOR REGENERATIVO

\section{- Agradecimientos}



A mis Directores, Guillermo y Osvaldo, por sus enseñanzas, su invaluable dedicación y motivación, así como por su calidez humana. Gracias por guiarme durante esta etapa y por ser un ejemplo en lo profesional y en lo personal.

Al Centro de Investigación y DesarRollo en Ciencias APlicadas “DR. Jorge J. Ronco” y a la FACULTAD DE INGENIERÍA DE LA UNIVERSIDAD NACIONAL DE LA PLATA.

Al Consejo Nacional de InVEstigaciones CientífiCAS y TÉCniCAS por su apoyo económico.

A Lucho, por dejarme ser partícipe en algunas de sus actividades en el INIFTA, introduciéndome así a este ámbito.

A todos los integrantes del grupo de trabajo, con los que tanto tiempo he compartido; en especial a Roberto, por su compañia, amistad y por tantos debates, tanto de indole académica como personal.

A Caro, por acompañarme, contenerme (y soportarme) durante todo este periodo. Sin duda lo mejor y más valioso que me dejó esta etapa en el plano no académico. Sin ella a mi lado, nada hubiera sido lo mismo.

A Augusto, a quien tuve la suerte de tener como docente en el comienzo de la carrera de grado y de mi carrera docente y quien me motivó a que siguiera este camino. Probablemente no estaría escribiendo estas palabras si no hubiéramos tenido aquellas charlas "post Matemática A", que siempre tendré presentes.

A mis amigos Gero, Gabriel, Israel, Juan y Rodri, personas incondicionales que estuvieron siempre a mi lado, escuchándome y aconsejándome tanto antes como durante el desarrollo de esta etapa.

A toda mi familia por acompañarme durante todo este tiempo y por motivarme a seguir adelante. En especial a mi papá y a mi mamá, que siempre me apoyaron y aconsejaron en mis decisiones. 
COMBUSTIÓN CATALÍTICA DE VOCS

UTILIZANDO SISTEMAS AUTOTÉRMICOS CON

INTERCAMBIO DE CALOR REGENERATIVO

\section{- Resumen}



La combustión catalítica constituye una de las mejores alternativas para la purificación de corrientes de aire que contienen bajas concentraciones de compuestos orgánicos volátiles (VOCs).

Las corrientes industriales a purificar se encuentran usualmente a una temperatura cercana a la ambiente y, en la mayoría de los casos prácticos, los caudales que se deben procesar son muy elevados. Por otro lado, dependiendo de las sustancias a tratar y del catalizador empleado, la temperatura necesaria para la combustión catalítica se encuentra típicamente en el intervalo de 200 a $400{ }^{\circ} \mathrm{C}$. De esta manera, surge la necesidad de emplear esquemas de combustión que permitan recuperar la energía de los gases tratados de forma eficiente.

Con dicho fin, resultan particularmente atractivos los sistemas de combustión autotérmicos (la energía necesaria para elevar la temperatura de la corriente a tratar proviene de la corriente efluente) con intercambio de calor regenerativo. En este tipo de sistemas, el intercambio de energía se realiza mediante un sólido que almacena la energía de la corriente caliente durante un período y la cede a la corriente fría en el siguiente período (a diferencia del intercambio de tipo recuperativo, el cual se realiza en forma directa, actuando el sólido presente como superficie de separación únicamente).

Teniendo en cuenta los aspectos mencionados en los párrafos anteriores, en la presente Tesis se llevó a cabo el análisis de tres tipos de sistemas de combustión autotérmicos con intercambio de calor regenerativo. Dos de ellos corresponden a los sistemas conocidos como de inversión de flujo, en los cuales se opera un lecho (adiabático) invirtiendo el sentido de circulación en forma periódica. En particular, se analizó un esquema convencional (sistema estático con inversión mediante válvulas) y un esquema rotativo (sistema móvil, donde todo el conjunto gira sobre un eje). El tercer esquema analizado está constituido por un intercambiador de calor rotativo (regenerador) y un reactor adiabático convencional. Cabe destacar que ambos sistemas de inversión de flujo, así como el regenerador, operan bajo un estado transitorio, pero con ciclos que se repiten periódicamente.

A los efectos de llevar a cabo el estudio de cada uno de los esquemas considerados, se consideró una corriente contaminada con etanol y acetato de etilo (contaminantes provenientes de industrias de impresión de envases), se pro- 
puso un modelo unidimensional heterogéneo y se desarrollaron métodos de cálculo para su implementación en un simulador del proceso. El mismo resultó suficientemente estable y preciso para el análisis de una amplia gama de condiciones.

Con los simuladores desarrollados se estudió la respuesta de cada sistema a diferente tipo de variaciones en las condiciones operativas y de diseño (disposición estructural, proporción entre las regiones inertes y la catalítica).

Finalmente, se propusieron diferentes diseños para cada esquema, considerando que en todos los casos dichos diseños debían tener la capacidad de cubrir una disminución en el contenido total de VOCs de hasta la mitad del contenido considerado como nominal. El análisis realizado permitió verificar que los tres sistemas propuestos son aptos para el tratamiento de la corriente considerada. En particular, tanto el esquema de inversión de flujo rotativo como el esquema conformado por el regenerador y el reactor adiabático se posicionaron como las alternativas más ventajosas. 
COMBUSTIÓN CATALÍTICA DE VOCS

UTILIZANDO SISTEMAS AUTOTÉRMICOS CON

INTERCAMBIO DE CALOR REGENERATIVO

- Índice general 

Introducción

1.1 Objetivos para el desarrollo de la Tesis $-4-$

1.2 Descripción del contenido de la Tesis $-5-$

- Técnicas de tratamiento de VOCs.................................................. - 7 -

2.1 Técnicas de destrucción de voCs..................................... 9 -

2.1.1 Combustión ........................................................ 9 -

2.1.1.1 Combustión térmica......................................... - 12 -

2.1.1.2 Combustión catalítica............................................ - 13 -

2.1.1.3 Sistema de inversión de flujo ................................... 15 -

2.1.1.4 Red de reactores ............................................ 18 -

2.1.2 Biofiltración ....................................................... 19 -

2.1.3 Fotocatálisis ..................................................... 21 -

2.2 Técnicas de recuperación de Vocs................................ 22 -

2.2.1 Adsorción ............................................................ 22 -

2.2.2 Absorción ......................................................... 25 -

2.2.3 Condensación .................................................... 25 -

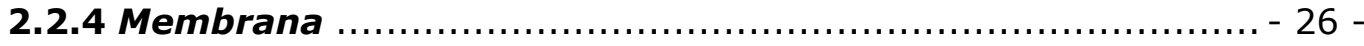

2.3 Características del sistema a tratar; fundamentación de la selección del conjunto de técnicas...................................... 27 -

2.3.1 Características de la corriente a tratar ....................... 28 -

2.3.2 Verificación de la selección de la técnica .......................... 29 -

2.3.3 Selección entre oxidación térmica y catalítica y esquemas de intercambio .............................................. 30 -

Modelado

3.1 Descripción de los esquemas a estudiar 
3.3 Modelo para el sistema de inversión de flujo

3.3.1 Condiciones iniciales y de contorno para el sistema de inversión de flujo convencional

3.3.2 Condiciones iniciales y de contorno para el sistema de inversión de flujo rotativo $-48-$

3.3.3 Pérdida de carga $-49-$

3.3.4 Contenido de VOCs a la salida del sistema $-50-$

3.3.5 Discusión del modelo $-51-$

3.3.5.1 Análisis particular del sistema rotativo $-56-$

3.4 Modelo para el sistema regenerador-reactor $-57-$

3.4.1 Modelo para el regenerador $-59-$

3.4.2 Modelo para el reactor $-61-$

3.4.3 Pérdida de carga $-63-$

3.4.4 Contenido de VOCs a la salida del sistema $-63-$

3.4.5 Discusión del modelo $-63-$

3.5 Aproximación de los sistemas de inversión de flujo para bajos tiempos de ciclo: reactor en contra-corriente

3.6 Conclusiones y comentarios finales $-68-$

Apéndice 3.1: Propiedades físicas y coeficientes de transferencia de materia y energía. $-71-$

Densidad $-71-$

Viscosidad $-71-$

Calor específico $-71-$

Calor específico medio $-72-$

Conductividad térmica $-73-$

Coeficientes de difusión molecular $-73-$

Entalpías de combustión $-74-$

Coeficientes de transferencia $-76-$

Apéndice 3.2: Parámetros geométricos del sistema $-79-$

Apéndice 3.3: Similitud entre el regenerador $y$ un intercambiador en contra-corriente para bajos tiempos de ciclo 
- Implementación numérica

4.1 Velocidad de reacción efectiva.................................. 87 -

4.2 Determinación de caudales de limpieza ............................ 91 -

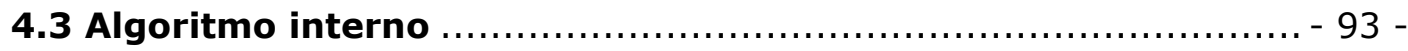

4.3.1 Desempeño del algoritmo interno........................... - 96 -

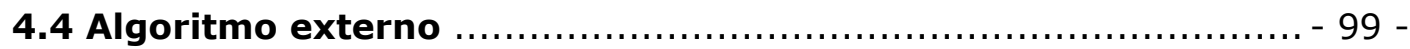

4.4.1 Criterios de corte .............................................. $103-$

4.4.2 Desempeño del algoritmo externo ........................... - 104 -

4.5 Algoritmo para el reactor en contra-corriente .................. - 105 -

4.6 Conclusiones y comentarios finales ............................. 106 -

Análisis de los sistemas de inversión de flujo ................................... 109 -

5.1 Consideraciones generales. Análisis preliminar mediante el

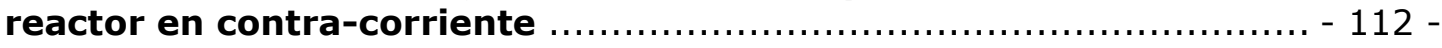

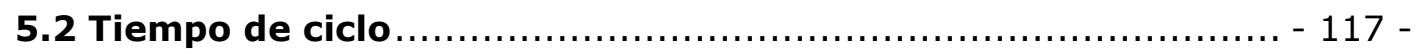

5.2.1 Comparación de los perfiles para $t_{\text {ciclo }}=10 \mathrm{~s}$ con los

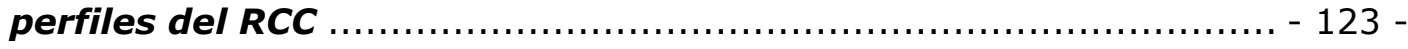

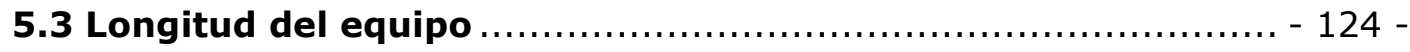

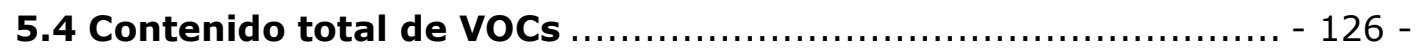

5.5 Caída en la actividad del catalizador............................. - 128 -

5.6 Aspectos adicionales ............................................. $130-$

5.7 Conclusiones y comentarios finales ............................ 133 -

Análisis del sistema regenerador-reactor................................. 135 -

6.1 Fracción de limpieza ............................................. $140-$

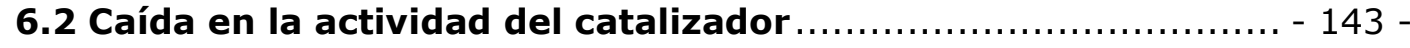

6.3 Conclusiones y comentarios finales ............................ 147 -

Apéndice 6.1: Análisis cualitativo del esquema autotérmico ........ - 149 - 
Diseño de los sistemas de inversión de flujo ................................ 155 -

7.1 Diseño del esquema convencional ............................... 158 -

7.1.1 Determinación de los rangos de búsqueda ................... - 163 -

7.1.1.1 Cota inferior de volumen y diámetro del equipo ................ - 164 -

7.1.1.2 Cota superior de volumen y diámetro del equipo ............... - 167 -

7.1.2 Diseños resultantes para cada configuración ................. - 170 -

7.1.2.1 Análisis complementario de los diseños obtenidos ............. - 173 -

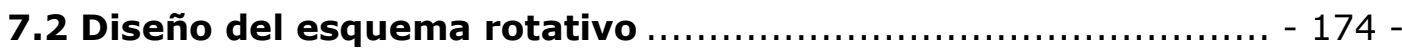

7.2.1 Cotas inferior $y$ superior para el volumen y el diámetro del equipo .................................................................... 178 -

7.2.2 Diseños resultantes para cada configuración ................. - 179 -

7.2.2.1 Análisis complementario de los diseños obtenidos ............ - 181 -

7.3 Conclusiones y comentarios finales .............................. $182-$

Diseño del sistema regenerador-reactor...................................... - 185 -

8.1 Análisis de grados de libertad y estrategia de diseño …........ - 187 -

8.1.1 Determinación de los rangos de búsqueda ................... - 192 -

8.1.1.1 Cotas inferiores y superiores para el regenerador ............ - 192 -

8.1.1.2 Cotas inferiores y superiores para el reactor ................... - 195 -

8.2 Justificación de la elección de la temperatura de alimentación al reactor..................................................... - 197 -

8.3 Diseños resultantes para cada configuración ...................... 201 -

8.3.1 Análisis complementario de los diseños obtenidos....... - 203 -

8.4 Conclusiones y comentarios finales ............................... $205-$

- Conclusiones finales: comparación. Síntesis de resultados y recomendaciones ......................................................................... 207 -

9.1 Análisis de los diseños obtenidos para cada esquema .......... 209 -

9.1.1 Comportamiento frente a la disminución de la actividad catalítica ......................................................................... - 213 - 
9.2 Síntesis de resultados alcanzados

9.2.1 Simulación de la operación de cada sistema $-217-$

9.2.2 Sistemas de inversión de flujo $-219-$

9.2.2.1 Diseño de los sistemas de inversión de flujo $-222-$

9.2.3 Sistema regenerador-reactor $-223-$

9.2.3.1 Diseño del sistema regenerador-reactor $-225-$

9.2.4 Comparación entre los esquemas estudiados $-226-$

9.3 Recomendaciones para futuros trabajos $-228-$

- Nomenclatura $231-$

- Referencias $243-$ 

COMBUSTIÓN CATALÍTICA DE VOCS

UTILIZANDO SISTEMAS AUTOTÉRMICOS CON

INTERCAMBIO DE CALOR REGENERATIVO

- Índice de Figuras 

Figura 2.1: $\quad$ esquema de un sistema de combustión.

Figura 2.2: $\quad$ esquema de un reactor catalítico de inversión de flujo convencional y perfiles típicos de temperatura y concentración al final de dos semiciclos consecutivos en el estado estacionario cíclico.

Figura 2.3: $\quad$ red de reactores constituida por 2 lechos.

Figura 2.4: $\quad$ red de reactores constituida por 3 lechos.

Figura 2.5: $\quad$ sistema de biofiltración.

Figura 2.6: $\quad$ sistema de adsorción.

Figura 2.7: $\quad$ rango de condiciones operativas para distintas técni29 cas de tratamiento de VOCs.

Figura 3.1: $\quad$ esquema del sistema de inversión de flujo rotativo.

Figura 3.2: $\quad$ esquema del sistema regenerador-reactor.

Figura 3.3: representación esquemática de la posición angular de una serie de canales.

Figura 3.4: $\quad$ reactor hipotético con el gas en contra-corriente.

Figura A3.2.1: representación de una celda de monolito.

Figura 4.1: representación esquemática de la incorporación de puntos adicionales en los primeros puntos para un esquema $\mathrm{DFA}_{3}$. 
Figura 4.2: $\quad$ variables en el css y tiempo de cálculo requerido para diferentes órdenes y número de intervalos.

Figura 4.3: tiempo de cálculo requerido frente a los errores en las predicciones de las variables en el css para diferentes órdenes del esquema DFA.

Figura 4.4: $\quad$ respuesta durante la predicción del css para distin104 tos valores del parámetro $M$.

Figura 5.1: $\quad$ efecto de la fracción de inerte en el lecho según el RCC.

Figura 5.2: $\quad$ reactor hipotético con el gas en contra-corriente.

Figura 5.3: $\quad$ efecto del tiempo de duración del ciclo en el sistema convencional.

Figura 5.4: $\quad$ perfiles de temperatura y fracción molar en el sistema de inversión de flujo convencional sin inerte, para el css. Sentido de flujo: de izquierda a derecha $\left(t_{\text {ciclo }}=100 \mathrm{~s}\right)$.

Figura 5.5: $\quad$ perfiles de temperatura y fracción molar en el sistema de inversión de flujo convencional sin inerte, para el css. Sentido de flujo: de izquierda a derecha $\left(t_{\text {ciclo }}=200 \mathrm{~s}\right)$.

Figura 5.6: $\quad$ perfiles de temperatura en la fase sólida y de acetato de etilo en la fase gaseosa para diferentes tiempos. Sentido de flujo: de izquierda a derecha $\left(t_{\text {ciclo }}=200 \mathrm{~s}\right)$. 
Figura 5.7: $\quad$ perfiles de temperatura y fracción molar en el sistema de inversión de flujo convencional con un $75 \%$ de región inerte, para el css (las lineas punteadas verticales indican los limites inerte-catalizador). Sentido de flujo: de izquierda a derecha $\left(t_{\text {ciclo }}=100 \mathrm{~s}\right)$.

Figura 5.8: $\quad$ perfiles de temperatura y fracción molar en el sistema de inversión de flujo convencional con un $75 \%$ de región inerte, para el css (las líneas punteadas verticales indican los límites inerte-catalizador). Sentido de flujo: de izquierda a derecha $\left(t_{\text {ciclo }}=200 \mathrm{~s}\right)$.

Figura 5.9: $\quad$ efecto del tiempo de duración del ciclo en el sistema 122 rotativo $\left(f_{L}=2 \%\right)$

Figura 5.10: comparación entre los perfiles de temperatura y fracción molar según el RCC y el modelo completo. Sentido de flujo: de izquierda a derecha $\left(t_{\text {ciclo }}=10 \mathrm{~s}\right)$.

Figura 5.11: efecto de la longitud total del equipo en el sistema 125 convencional $\left(t_{\text {ciclo }}=120 \mathrm{~s}\right)$.

Figura 5.12: efecto del contenido total de VOCs en el sistema convencional (en términos del $\Delta T_{a d} ; t_{\text {ciclo }}=120 \mathrm{~s}$ ).

Figura 5.13: $\quad$ efecto de la caída en la actividad del catalizador en el 129 sistema convencional $\left(t_{\text {ciclo }}=120 \mathrm{~s}\right)$.

Figura 5.14: $\quad$ efecto de la caída en la actividad del catalizador se130 gún el modelo $\operatorname{RCC}\left(f_{I}=50 \%\right)$.

Figura 5.15: efecto de la composición de VOCs en la alimentación para $\Delta T_{a d}=40^{\circ} \mathrm{C}\left(t_{\text {ciclo }}=120 \mathrm{~s}\right)$. 
Figura 6.1: efecto de la fracción de limpieza en el sistema regenerador-reactor.

Figura 6.2: $\quad$ efecto de la caída en la actividad del catalizador en el sistema regenerador-reactor $\left(f_{L}=1 \%\right)$.

Figura 6.3: $\quad$ efecto del tiempo de ciclo y la longitud del regenerador sobre la actividad crítica del catalizador $\left(f_{L}=1 \%\right)$.

Figura 6.4: actividad critica del catalizador vs. la temperatura de alimentación al reactor $\left(f_{L}=1 \%\right)$.

Figura A6.1.1: solución al conjunto de Ecuaciones (A6.1.9) a (A6.1.12) para el caso base.

Figura A6.1.2: $\quad$ conjunto de soluciones obtenidas al disminuir la capacidad de intercambio.

Figura A6.1.3: conjunto de soluciones obtenidas al disminuir la actividad del catalizador.

Figura 7.1: $\quad$ respuesta del sistema de inversión de flujo convencional frente a la variación de $t_{\text {ciclo }}$ para el contenido de VOCs nominal y para cada uno de los diseños alcanzados.

Figura 7.2: $\quad$ efecto de la fracción de limpieza sobre la pérdida de carga en el sistema de inversión de flujo rotativo (configuración 1). 
Figura 7.3: $\quad$ efecto de la fracción de limpieza sobre la temperatura máxima y la emisión de VOCs en el sistema de inversión de flujo rotativo (configuración 1).

Figura 7.4: $\quad$ respuesta del sistema de inversión de flujo rotativo frente a la variación de $t_{\text {ciclo }}$ para el contenido de VOCs nominal y para cada uno de los diseños alcanzados.

Figura 8.1: efecto de la temperatura de alimentación al reactor 199 sobre el dimensionamiento de ambos equipos (regenerador y reactor), para la configuración 1.

Figura 8.2: $\quad$ efecto de la temperatura de alimentación al reactor 199 sobre la masa total de inerte y catalizador, para la configuración 1 .

Figura 8.3: efecto de la temperatura de alimentación al reactor 200 sobre los valores de $t_{\text {ciclo }}$ (operativo y crítico) para la condición con el contenido de VOCs nominal y sobre la actividad crítica para la condición con el contenido de VOCs mínimo.

Figura 8.4: $\quad$ respuesta del sistema regenerador-reactor frente a la 204 variación de $t_{\text {ciclo }}$ para el contenido de VOCs nominal y para cada uno de los diseños alcanzados.

Figura 9.1 esquema de inversión de flujo rotativo con retorno. 
COMBUSTIÓN CATALÍTICA DE VOCS

UTILIZANDO SISTEMAS AUTOTÉRMICOS CON

INTERCAMBIO DE CALOR REGENERATIVO

- Índice de Tablas 

Tabla 2.1: $\quad$ características de la corriente a tratar.

Tabla 3.1: $\quad$ propiedades del catalizador.

Tabla 3.2: $\quad$ propiedades de la cordierita.

Tabla 3.3: $\quad$ parámetros cinéticos.

Tabla A3.1.1: volúmenes de difusión.

Tabla A3.1.2: coeficientes para el cálculo de las entalpías de combustión.

Tabla A3.1.3: parámetros para el cálculo de los coeficientes de transferencia.

Tabla 4.1: $\quad$ coeficientes de combinación para el método DFA.

Tabla 4.2: $\quad$ parámetros utilizados para el estudio de los algoritmos.

Tabla 5.1: $\quad$ parámetros utilizados para el estudio de los sistemas de inversión de flujo.

Tabla 6.1: $\quad$ parámetros utilizados para el estudio del sistema regenerador-reactor.

Tabla 6.2: $\quad$ valores del caudal de limpieza, fracción de limpieza para el máximo local en $T_{G}^{0, R}$ y de la relación entre el caudal de limpieza y el total en la región de calentamiento del sólido, para los diferentes tiempos de ciclo empleados. 
Tabla 7.1: $\quad$ características de la corriente a tratar.

Tabla 7.2: $\quad$ configuraciones de celda y propiedades de las mismas.

Tabla 7.3: $\quad$ cotas inferiores y superiores para el volumen y diámetro del equipo en el sistema de inversión de flujo convencional.

Tabla 7.4: resumen de resultados obtenidos en el dimensionamiento del esquema de inversión de flujo convencional para diferentes configuraciones de celda, según la propuesta de diseño dada en la Sección 7.1. Tiempo de ciclo mínimo: $240 \mathrm{~s}$.

Tabla 7.5: $\quad$ parámetros utilizados para el estudio del efecto de la fracción de limpieza sobre el esquema de inversión de flujo rotativo.

Tabla 7.6: $\quad$ cotas inferiores y superiores para el volumen y diámetro del equipo en el sistema de inversión de flujo rotativo.

Tabla 7.7: $\quad$ resumen de resultados obtenidos en el dimensionamiento del esquema de inversión de flujo rotativo para diferentes configuraciones de celda, según la propuesta de diseño dada en la Sección 7.2. Tiempo de ciclo mínimo: $30 \mathrm{~s}$.

Tabla 8.1: $\quad$ características de la corriente a tratar.

Tabla 8.2: $\quad$ configuraciones de celda y propiedades de las mis188 mas. 
Tabla 8.3: $\quad$ cotas inferiores y superiores para el volumen y diámetro del regenerador

Tabla 8.4: $\quad$ cotas inferiores y superiores para el volumen y diá197 metro del reactor

Tabla 8.5: $\quad$ resumen de resultados obtenidos en el dimensiona202 miento del esquema regenerador-reactor para diferentes configuraciones de celda, según la propuesta de diseño dada en la Sección 8.1.

Tabla 9.1: $\quad$ características principales de los diseños alcanzados para cada esquema.

Tabla 9.2: $\quad$ valores de la mínima actividad catalítica tolerable en cada sistema, según los diseños obtenidos, para la condición de trabajo con el contenido de VOCs mínimo.

Tabla 9.3: emisión promedio de VOCs y temperatura máxima en cada sistema, según los diseños obtenidos, para la operación con el contenido de VOCs nominal, habiendo reducido la actividad catalítica a $1 / 5$ del valor correspondiente al catalizador fresco. 


\section{Capítulo 01}

\section{- Introducción}

- Objetivos para el desarrollo de la Tesis

- Descripción del contenido de la Tesis 

El desmedido aumento en la contaminación del aire durante las últimas décadas, ha convertido al control de la polución en uno de los objetivos prioritarios a analizar desde la investigación, el desarrollo de tecnologías y la puesta en marcha de regulaciones ambientales. Entre los contaminantes del aire más comunes, se encuentran los compuestos orgánicos volátiles (también denominados VOCs por sus siglas en inglés), los cuales pueden ser de origen natural (cloroplastos vegetales) o antropogénicos (emisiones fugitivas de escape, procedentes de fuentes móviles o estacionarias). Las plantas industriales representan gran parte de las fuentes de escape estacionarias.

Los procesos de impresión en industrias elaboradoras de envases requieren una ventilación continua del ambiente de trabajo. Esto ocasiona una importante contaminación del aire, debido a la evaporación de los solventes utilizados, entre los que se encuentran el acetato de etilo y el etanol. Esta situación requiere la purificación de la corriente de aire conteniendo estos compuestos en baja cantidad y constituye el caso base sobre el cual se realizará este estudio.

Las técnicas de tratamiento de efluentes gaseosos pueden clasificarse en dos grandes grupos. Por un lado, se puede identificar a las medidas que se centran en la modificación del proceso o los equipos (medidas preventivas), que incluyen mejoras en el proceso, desde la inspección y el mantenimiento para evitar fugas, hasta el rediseño completo de las instalaciones para minimizar los productos orgánicos residuales. Por otro lado, se encuentran las medidas que implican algún método de tratamiento de las emisiones de VOCs (medidas correctivas), clasificándose normalmente en tecnologías de eliminación y tecnologías de recuperación. Si bien la conveniencia del uso de una $u$ otra opción sería determinada en última instancia por el objetivo específico de la aplicación y/o un análisis económico del proceso (Khan y Goshal, 2000), en términos generales el nivel de concentración de los VOCs y el caudal de la corriente gaseosa permitirán realizar una primera selección. Así por ejemplo, las tecnologías de recuperación serán más atractivas cuando el contenido de VOCs en la corriente a tratar sea elevado, mientras que para el caso opuesto serán más apropiadas las tecnologías de eliminación.

Tanto el grupo de tecnologías de recuperación como el de eliminación, posee una amplia gama de alternativas de acuerdo al proceso involucrado (o conjun- 
to de ellos). Si bien en una primera instancia, la elección entre estas alternativas se puede realizar a través de diagramas que delimitan regiones de concentración y caudal donde cada una de ellas es viable, ya sea desde el aspecto técnico o económico, en este trabajo se limitará el análisis a algunas variantes dentro de las tecnologías de oxidación catalítica (eliminación). Esta elección está motivada por la intención de dar continuidad a acciones previas del grupo de investigación, con resultados que indican ventajosas posibilidades de aplicación (Campesi y col., 2007; Campesi, 2012). No obstante, como se verá en el Capítulo 2, la incineración catalítica resulta una de las opciones más adecuadas para el presente caso. En este contexto, la modalidad empleada para elevar la temperatura de la corriente a tratar hasta la temperatura de reacción y la eventual recuperación de energía de los gases ya tratados resulta un aspecto de alta significación económica.

\subsection{Objetivos para el desarrollo de la Tesis}

El objetivo general de la Tesis es analizar distintos sistemas de eliminación de compuestos orgánicos volátiles por medio de la oxidación catalítica de los mismos y desarrollar criterios para discriminar las alternativas más convenientes desde el punto de vista técnico-económico.

Se estudiará en forma específica la incineración catalítica de acetato de etilo y etanol en corrientes efluentes de plantas de impresión de envases, bajo tres variantes:

* reactor de inversión de flujo con inversión mediante válvulas (esquema convencional; estático);

* reactor de inversión de flujo con inversión mediante la rotación sobre un eje (esquema rotativo; móvil);

* reactor catalítico estacionario con precalentamiento de la corriente de aire mediante un intercambiador de calor rotativo (regenerador).

En este trabajo se pretende analizar en primer lugar, las similitudes y diferencias que surjan de las tres variantes antes mencionadas, considerando que todas comparten una característica común: los esquemas de cada una de ellas 
constituyen un sistema autotérmico con intercambio de calor regenerativo. En segundo lugar, se busca establecer un criterio de diseño para cada una de las variantes que brinde flexibilidad en la operación frente a variaciones en el contenido de VOCs y un criterio de comparación que permita identificar ventajas y desventajas de cada una.

La obtención y análisis de resultados se realiza mediante la simulación de cada una de las variantes propuestas. Para ello, se estudian distintas metodologías de cálculo que permitan mantener un equilibrio entre el tiempo de cálculo requerido y la complejidad de las mismas.

\subsection{Descripción del contenido de la Tesis}

En el Capítulo 2 se realiza una descripción breve de las diferentes técnicas de tratamiento de VOCs, tanto de las recuperativas como las de eliminación. A partir de la definición de las propiedades operativas de la corriente a tratar, se justifica la selección del tipo de sistema a utilizar.

En los Capítulos 3 y 4 se cubren los aspectos relacionados a la simulación de cada una de las variantes, modelos elegidos, cinética de las reacciones involucradas, cálculo de propiedades fisicas y algoritmos de resolución utilizados. Adicionalmente, en el Capítulo 3 se presenta una aproximación al sistema de inversión de flujo convencional cuando este opera a altas frecuencias de inversión, que resulta de gran utilidad para explicar algunos comportamientos observados en el sistema real.

En los Capítulos 5 y 6 se estudia el comportamiento de cada uno de los esquemas de tratamiento seleccionados frente a variaciones de diferentes parámetros operativos, con la finalidad de esclarecer ciertos aspectos distintivos de los mismos. Particularmente, se estudian variaciones en: el tiempo de ciclo, la composición y el contenido total de VOCs, la caída de la actividad catalítica.

En el Capítulo 7 se cubre el diseño de ambos esquemas de inversión de flujo propuestos, mientras que en el Capítulo 8 se aborda el diseño de los equipos en el sistema compuesto por el reactor catalítico estacionario y el intercambiador de calor rotativo. Para ello se propone una estrategia de diseño para cada caso, apoyándose en los análisis realizados en los Capítulos 5 y 6 y 
teniendo presente que los sistemas utilizados deberán poseer la flexibilidad suficiente para soportar variaciones en el contenido de VOCs de la corriente a tratar.

En el Capítulo 9 se presentan las conclusiones finales del presente estudio, comparando los esquemas estudiados entre sí, a partir de los diseños alcanzados en los Capítulos 7 y 8. Finalmente, se presenta una síntesis de los resultados alcanzados y se proponen algunas recomendaciones para trabajos futuros. 


\section{Capítulo 02}

\section{- Técnicas de tratamiento de VOCs}

- Técnicas de destrucción de VOCs

- Técnicas de recuperación de VOCs

- Características del sistema a tratar. Fundamentación de la selección del conjunto de técnicas 

Como se mencionó en el Capítulo 1, existe una amplia variedad de alternativas para el tratamiento de VOCs. Considerando que el conocimiento de los fundamentos de cada una de ellas constituye el punto inicial de cualquier estudio relacionado al tratamiento de VOCs en corrientes de aire, se comenzará con una revisión general de los mismos. Si bien existe una abundante bibliografia al respecto, la mayor parte de los trabajos abordan aspectos específicos de cada una de las técnicas disponibles. Por esta razón, se utilizarán las descripciones realizadas en un conjunto de trabajos de revisión (Everaert y col., 2002; Moretti; 2002; Khan y Ghoshal, 2000; de la Fuente Garcia y Gutiérrez Martin, 1998).

\subsection{Técnicas de destrucción de VOCs}

Como su nombre lo indica, el objetivo de este conjunto de técnicas es eliminar los VOCs presentes en la corriente a tratar. Las alternativas comunes en este caso son la combustión, tanto térmica como catalítica, la biofiltración o un proceso fotocatalítico.

\subsubsection{Combustión}

La combustión (o incineración) es el método destructivo más importante en la actualidad. La idea principal de esta alternativa es destruir los VOCs presentes en la corriente a partir de una reacción de oxidación, con o sin catalizador presente (combustión térmica o catalítica). Bajo las condiciones adecuadas, los productos de reacción serán normalmente $\mathrm{CO}_{2}$ y $\mathrm{H}_{2} \mathrm{O}$, los cuales son relativamente inocuos, haciendo a la combustión un método atractivo de disposición de residuos. Sin embargo, cuando estén presentes en la mezcla compuestos halogenados (con un halógeno "X") o que contengan azufre, los productos de la combustión completa incluirán además los componentes ácidos $\mathrm{HX} \mathrm{y} \mathrm{SO}_{2}$, respectivamente. En estos casos, se requerirá la remoción de los componentes ácidos por medio de un absorbedor.

Cualquiera sea el tipo, para que la combustión se lleve a cabo se debe elevar la temperatura de la corriente hasta un valor suficiente, de forma tal que la energía aportada por la propia reacción exceda las pérdidas de calor y con 
ello se autosostenga. De esta manera, cualquier mezcla de compuestos orgánicos y aire se encenderá si la temperatura es llevada a un nivel suficientemente alto. Esta temperatura se conoce como temperatura de ignición y, para un componente y concentración dados, dependerá fuertemente de la manera en que la combustión se lleva a cabo, ya sea por la vía térmica o catalítica, marcando esto la principal diferencia entre ambas posibilidades.

En su concepción más elemental, estos sistemas constan de tres estructuras básicas: un precalentador (quemador), para iniciar y, dependiendo el tipo de operación, mantener la reacción; una cámara de combustión o un reactor catalítico; un sistema de intercambio de energía, cuya función usual es precalentar la corriente de entrada o eventualmente generar vapor.

La presencia o no de un sistema de intercambio y la forma en que el mismo se realiza en caso de estar presente, abre un abanico de posibilidades que resulta independiente del tipo de combustión.

La primera alternativa es prácticamente exclusiva de los sistemas de combustión térmica. En estos casos el gas a tratar se calienta en forma directa al pasar por una llama que se mantiene con un combustible adicional. En algunas aplicaciones, si los VOCs poseen la concentración suficiente, pueden utilizarse directamente como combustible, que será inyectado en los quemadores.

Otra opción consiste en precalentar la corriente a tratar utilizando para ello un intercambiador de superficie convencional (recuperativo), normalmente de tipo compacto o eventualmente de tipo tubo y coraza. En general, el precalentamiento de la corriente trae aparejado una disminución de los costos operativos, pues disminuye el consumo de combustible adicional. Sin embargo, aumenta los costos fijos por la presencia del sistema de intercambio de energía.

Por último, se puede utilizar un sistema de intercambio de energía de tipo regenerativo, que difiere del recuperativo por la forma en que se lleva a cabo el intercambio. En el recuperativo, el intercambio de energía se realiza de una corriente a la otra en simultáneo, a través de una superficie de separación entre los fluidos. En el regenerativo, el intercambio de energía se realiza en forma indirecta (y alternada) a través de un sólido, de alta capacidad y resistencia térmica, que almacena la energía de la corriente caliente durante un periodo y 
la cede a la corriente fría en el otro (Willmott, 2002). Esto se puede lograr utilizando dos lechos paralelos trabajando en forma alternada, invirtiendo el sentido de circulación de la corriente en los lechos mediante válvulas especiales, operando a razón de 15 a 60 ciclos por hora (de la Fuente García y Gutiérrez Martin, 1998). Otra alternativa es utilizar un intercambiador rotativo tipo Ljungström. El mismo consiste en un lecho cilindrico con una matriz de relleno y una partición, de tal manera que se mantiene una posición espacial fija para las corrientes fría y caliente (se eliminan las válvulas) y gira el propio intercambiador, logrando así el contacto alternado de las corrientes fría y caliente con la matriz de relleno (Shah y Sekulic, 2003). En cualquiera de los casos, luego de un dado número de ciclos, el sistema de intercambio alcanzará un estado seudo-estacionario o estado estacionario cíclico (css, de sus siglas en inglés).

Las ventajas y desventajas de cada esquema radican en la relación de costos fijos y operativos, así como también en la relación entre rendimiento del sistema y la complejidad. Así, los sistemas de intercambio regenerativos resultan más eficientes que los recuperativos, especialmente para grandes volúmenes de aire y bajas concentraciones de VOCs, lo cual permite trabajar con bajo e incluso nulo consumo de combustible (de la Fuente García y Gutiérrez Martín, 1998). Por otro lado, los sistemas recuperativos poseen mayor sencillez operativa y alcanzan las condiciones de operación en tiempos más cortos que los regenerativos.

Para finalizar, en la Figura 2.1 se muestra un esquema conceptual de un sistema de combustión, térmica o catalítica. En la misma se incorpora una unidad absorbedora para contemplar aquellos casos en donde estén presentes compuestos halogenados o con azufre. También se incluye el combustible adicional y el aire para combustión que serán necesarios cuando se utilice el quemador. Finalmente, un aspecto que merece una discusión adicional es la inclusión del aire para dilución. Si bien es un problema poco significativo para el tratamiento de bajas cantidades de VOCs en aire, esto se incluye debido a que, por razones de seguridad, la máxima concentración de VOCs en la entrada al sistema debe ser sustancialmente menor que el limite de explosividad de los componentes ( $L E L$ de sus siglas en inglés). Dicho límite corresponde a la concentración mínima en aire capaz de mantener la combustión a la tempera- 
tura ambiente (Lewandowski, 2000). Como regla, se utiliza un factor de seguridad de $1 / 4$ cuando fuese necesaria su consideración (25\% del $L E L)$.

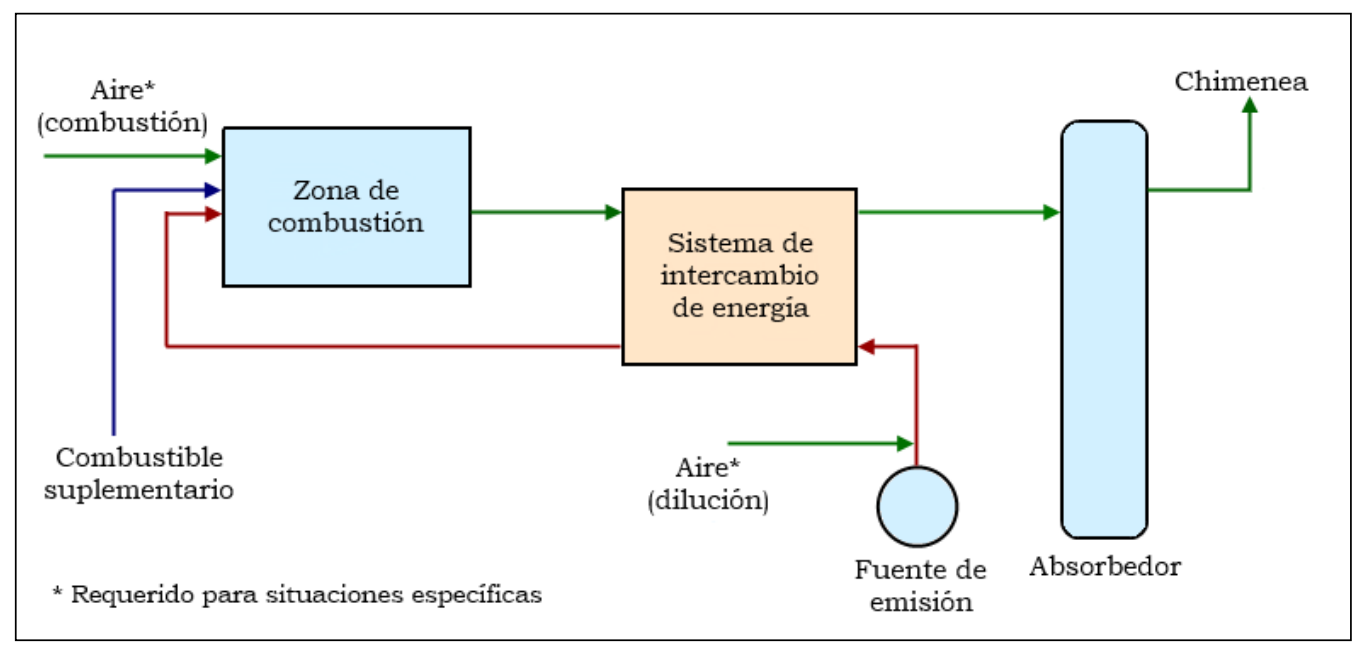

Figura 2.1: esquema de un sistema de combustión.

\subsubsection{Combustión térmica}

En los incineradores térmicos, la zona de combustión consta de un quemador, para iniciar y mantener la reacción y de una cámara de combustión, que es un recipiente con material refractario, prácticamente vacío y donde los gases se mezclan y terminan de reaccionar. En estos sistemas, la reacción de descomposición de los VOCs procede solo por temperatura, sin que intervenga un catalizador. Por esta razón, el nivel térmico de la corriente a tratar debe llevarse desde la temperatura a la cual se disponen la misma (usualmente la ambiente) hasta al menos la temperatura de ignición en fase homogénea. Como consecuencia, los incineradores térmicos trabajan normalmente a temperaturas por encima de $\operatorname{los} 800^{\circ} \mathrm{C}$, dependiendo del nivel de control requerido y del tiempo de residencia en la cámara de combustión. Por ejemplo, muchos incineradores térmicos están diseñados para proveer no más de un segundo de tiempo de residencia del gas a tratar, con temperaturas típicas entre 650 a $1000^{\circ} \mathrm{C}$ (Moretti, 2002).

El elevado nivel térmico requerido para la combustión térmica trae aparejado algunas desventajas que se mencionan a continuación: 
* Si se pretende recuperar energía de la corriente efluente para precalentar la corriente a tratar y se debe procesar un caudal muy elevado, junto con un contenido de VOCs muy bajo (baja fuerza impulsora), el volumen resultante de los intercambiadores puede ser prohibitivo, tanto desde un punto de vista técnico como económico.

* Cuando la concentración de los VOCs es muy baja, puede ser muy dificil mantener la autotermia del sistema, debido a que las altas temperaturas dificultan la aislación del sistema. Esto limita el mínimo contenido de VOCs que puede ser tratado.

* Se pueden formar $\mathrm{NO}_{\mathrm{X}}$, los cuales son muy perjudiciales para el medioambiente.

Para suplir los dos primeros problemas se recurre al uso de combustible adicional, con el consecuente aumento en los costos operativos. Para el tercer problema, se debe realizar un tratamiento ulterior de la corriente de salida de la cámara de combustión o bien mitigar su formación (Lewandoski, 2000).

\subsubsection{Combustión catalitica}

Desde el punto de vista constitutivo, la principal diferencia de la combustión catalítica frente a la térmica, radica en la presencia del reactor catalítico en lugar del conjunto quemador-cámara de combustión.

El uso de un catalizador permite disminuir sustancialmente la temperatura necesaria para la combustión, típicamente con valores menores a $450^{\circ} \mathrm{C}$ (Moretti, 2002), aunque este nivel de temperatura requerido depende fuertemente del tipo de VOC. Debido a esto, el precalentamiento de la corriente de gas a tratar suele llevarse a cabo únicamente con la corriente efluente del reactor, por medio de un intercambiador recuperativo de tipo compacto o por un intercambiador de calor regenerativo, tal como fuera descrito previamente. En la práctica sin embargo, el quemador no se elimina y es utilizado durante el arranque del equipo.

El sistema catalítico debe optimizarse en función de los requisitos de la aplicación, diseñando correctamente sus componentes: soporte y material activo (actividad, superficie específica, estabilidad, difusión de reactivos y 
productos). Los catalizadores típicamente usados para la eliminación de VOCs incluyen como materiales activos a los óxidos metálicos o metales nobles (Moretti, 2002).

Desde un punto de vista de diseño, la menor temperatura requerida para oxidar los VOCs de la corriente gaseosa conduce a intercambiadores más pequeños. En adición, genera un ahorro de energía (en comparación a la combustión térmica), debido a que no se utiliza el quemador previo al reactor durante la operación normal del sistema. Por otro lado, permite trabajar con menor cantidad de oxígeno, no genera $\mathrm{NO}_{\mathrm{x}} \mathrm{y}$ puede emplearse para caudales variables. La principal desventaja respecto de la oxidación térmica es el costo del catalizador (especialmente cuando el mismo contiene metales nobles) y su posible envenenamiento, ya sea por presencia de azufre en la carga o por deposición de materia orgánica en los poros (Khan y Ghoshal, 2000).

Por último, cabe aclarar que, aun cuando las temperaturas necesarias para la combustión catalítica son significativamente menores que para la combustión térmica, la primera puede no ser un proceso económicamente atractivo cuando los caudales a procesar son muy elevados y la concentración de los VOCs a tratar es muy baja. En tales circunstancias, tanto el volumen requerido de lecho catalítico como el área de intercambio necesaria en el intercambiador (especialmente si se pretende utilizar un intercambiador de superficie), pueden resultar muy elevados. Una forma de solventar este inconveniente es incorporar un sistema de adsorción/desorción previo a la entrada al reactor con el objetivo de concentrar los VOCs en la corriente para su posterior tratamiento en condiciones más favorables. Esta alternativa fue estudiada dentro del grupo de investigación por Campesi (2012), concluyéndose que la incorporación de dicha etapa de concentración resulta muy beneficiosa, permitiendo reducir los requerimientos de área de intercambio y volumen de reactor (en términos de masa de materiales a utilizar) a valores cercanos al $10 \%$ respecto de la situación sin etapa de concentración. Como contrapartida, para las condiciones de mayor concentración, se obtiene una temperatura de salida en el reactor catalítico que puede ser muy elevada para el material utilizado $\left(\approx 520^{\circ} \mathrm{C}\right)$. 


\subsubsection{Sistema de inversión de flujo}

El sistema de inversión de flujo consiste en la operación de un lecho, generalmente catalítico adiabático, en el cual se invierte el sentido de la corriente a tratar en forma periódica, alimentando la misma sin precalentamiento. De esta manera, el equipo es forzado a operar bajo condiciones transientes, con ciclos que se repiten periódicamente (css), explotando los beneficios del intercambio de energía regenerativo. En la Figura 2.2 se muestra un diagrama de este esquema, para un lecho catalítico con material inerte en los extremos, en donde la inversión se realiza mediante una válvula (esquema de inversión de flujo convencional).

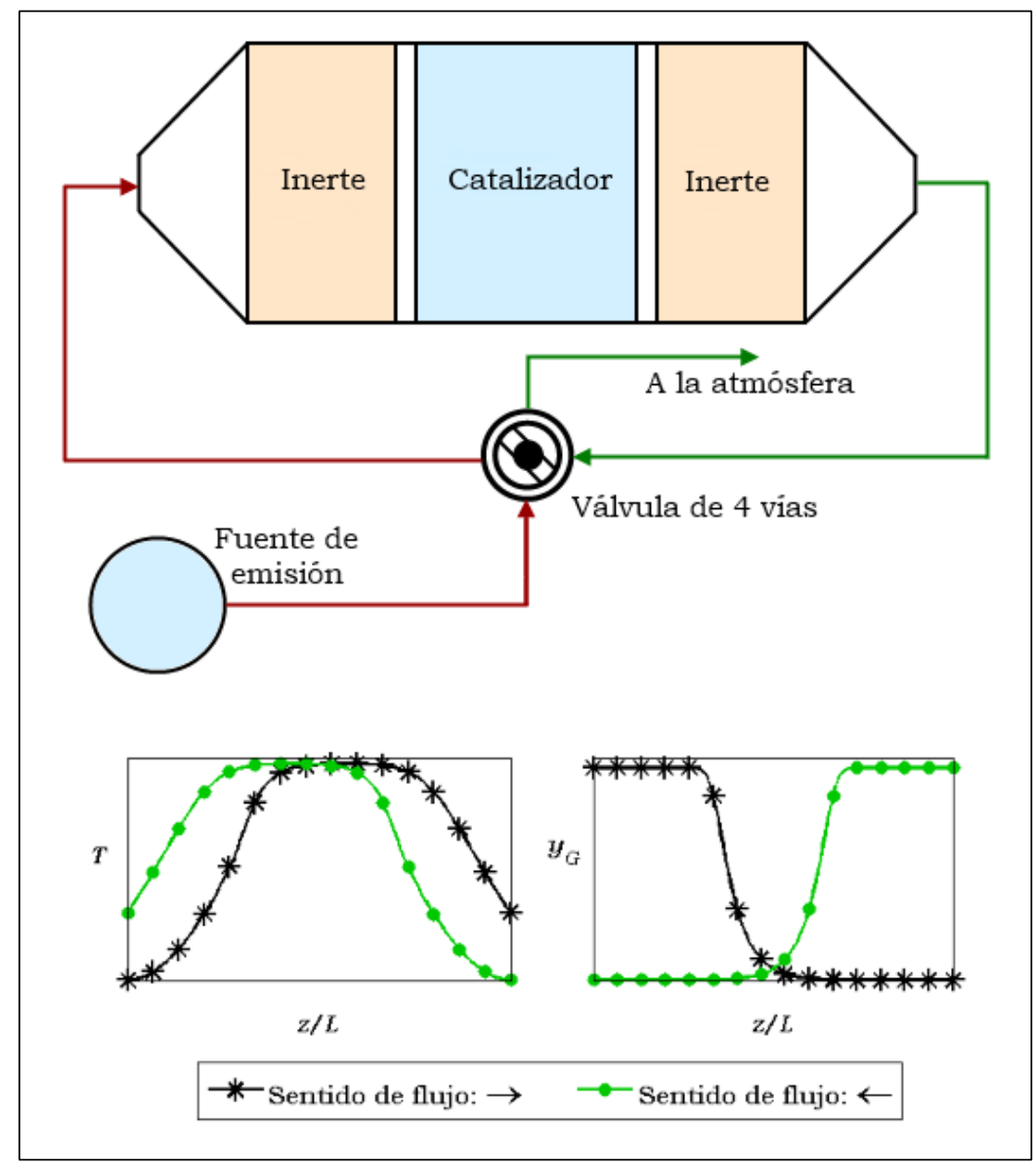

Figura 2.2: esquema de un reactor catalítico de inversión de flujo convencional y perfiles típicos de temperatura y concentración al final de dos semiciclos consecutivos en el estado estacionario cíclico. 
Si bien la mayor parte de los estudios están orientados a la operación de inversión de flujo en reactores catalíticos, estos sistemas pueden concebirse también para la incineración térmica. De hecho, es muy usual encontrar incineradores térmicos regenerativos comerciales que, a diferencia de los descritos en la Sección 2.1.1, funcionan con inversión de flujo en su totalidad y no solo en los lechos de intercambio. Estos equipos (conocidos también como reactores térmicos de inversión de flujo) se diseñan formando una "U" invertida, en donde las dos ramas verticales poseen los lechos de intercambio y la zona superior contiene el quemador y la cámara de combustión (Thermal Oxidizers, s.f.). No obstante, la descripción siguiente se centrará en los reactores de inversión de flujo catalíticos.

La primera referencia a estos esquemas data del año 1938 (Cottrell, 1938), pero a pesar de ello, este concepto no fue desarrollado hasta los años 80 (Matros, 1989; Boreskov y Matros, 1983) cuando se comenzó a estudiar para alcanzar la autotermia en sistemas reaccionantes en general, con muy limitada liberación de energía por reacción química. Sus principios de funcionamiento y sus aplicaciones siguen estudiándose activamente en la actualidad, principalmente en el tratamiento de VOCs (e.g., Chen y col., 2011; Marín y col., 2010), habiéndose posicionado fuertemente como una alternativa para este fin.

A los fines de visualizar conceptualmente la operación, se puede pensar qué ocurre si, habiendo precalentado el sistema hasta un nivel térmico para el cual la velocidad de reacción fuese apreciable, se comienza a alimentar la corriente a tratar a la temperatura en que se dispone la misma. En esta situación, habrá una pequeña región del lecho donde la corriente elevará su temperatura prácticamente sin reaccionar (enfriando el lecho), hasta alcanzar la temperatura de ignición, donde reaccionará liberando energía que será transferida al sólido. De esta manera, se formará un frente de onda frio que comenzará a moverse en el sentido de avance de la corriente gaseosa pero a una velocidad significativamente menor (Matros, 1989). Si se mantuviera el sentido de alimentación de la corriente, dicho frente de onda alcanzaría el otro extremo del reactor, habiendo enfriado todo el sistema y por ende "apagando" el mismo. En la práctica, el sentido de circulación se invierte antes de que la onda térmica alcance a enfriar una parte significativa de la región catalítica, iniciando así un nuevo semiciclo. En el mismo, se repetirá la secuencia descri- 
ta anteriormente y la zona que fue enfriada previamente será calentada por la corriente de gas que ha reaccionado. Al cabo de algunos ciclos, se habrá formado un perfil de temperatura tipo "campana” que se moverá a lo largo del sistema en uno u otro sentido, conforme se invierte el sentido de circulación de la corriente. De esta manera, se logra mantener la región catalíticamente activa a un nivel térmico elevado. Para visualizar mejor lo anterior, en la Figura 2.2 también se muestra un gráfico cualitativo de los perfiles de temperatura y concentración (fracción molar), al final de dos semiciclos consecutivos en el css.

En principio, el sistema no requiere la participación de ninguna operación adicional, por lo que evita la instalación de intercambiadores de calor y/o el uso de combustible adicional (con excepción del calentamiento inicial del lecho durante la puesta en marcha de la operación), porque el propio material catalítico participa del intercambio de energia en forma regenerativa. Esto último, generaría, en principio, que los lechos catalíticos sean más voluminosos que en otras opciones (como la incineración catalítica o un sistema combinado de concentración e incineración). Sin embargo, durante una elevada fracción del ciclo, ambas zonas extremas del lecho permanecen a una temperatura menor a la necesaria para que la reacción se lleve a cabo a velocidades apreciables. Por esta razón, los extremos del lecho se rellenan de material inerte, menos costoso que el catalizador. Esto permite aliviar el mayor requerimiento en volumen de catalizador, más aún si se tiene presente que en otros sistemas se deben instalar equipos adicionales (intercambiadores, entre otros). Sin embargo, se debe poner especial atención en la elección del material inerte, no solo por sus propiedades térmicas, sino porque debe evitar la adsorción de los VOCs, aspecto enfatizado por Zagoruiko (2008).

Una variante al esquema de inversión mediante válvulas, mostrado en la Figura 2.2, consiste en montar el reactor sobre un rotor, dividiendo la corriente a tratar en dos subcorrientes, alimentando cada una de ellas sobre la mitad de la sección del rotor y en sentido opuesto. De esta manera, se logra la inversión de flujo por la propia rotación del sistema y se elimina el uso de válvulas (Kolios y col., 2000). La principal diferencia entre estas alternativas radica en el tiempo de inversión mínimo admisible, limitado por la vida útil de la válvula en la primera (Bunimovich y Sapoundjiev, 2013; Barresi y col., 2007) y por la velocidad de rotación máxima en la segunda. 
Por último, es interesante reconocer la existencia de una gran similitud entre este tipo de operación y la incineración (térmica o catalítica) regenerativa, tal como fue descrita en la Sección 2.1.1; sin embargo, existe una diferencia que se debe señalar. En estos casos, si bien los lechos utilizados para el intercambio de energía operan con inversión de flujo, en la operación del reactor o del conjunto quemador-cámara de combustión, el sentido de alimentación de la corriente a tratar no se invierte. Cabe destacar que el uso de intercambiadores rotativos en lugar de lechos en paralelo, incorpora una segunda diferencia: la temperatura de alimentación al conjunto quemador-cámara de combustión o al reactor catalítico, resulta constante una vez alcanzado el css, con lo cual dichas regiones operan bajo un estado estacionario "puro".

\subsubsection{Red de reactores}

Si bien el reactor de inversión de flujo es el que ha recibido mayor atención en las últimas dos décadas, no es el único sistema que permite explotar las ventajas de la operación con intercambio de calor regenerativo y simultánea reacción química. Existen otras configuraciones que permiten operar el reactor bajo un estado transiente, pero cíclicamente estacionario. La diferencia con el esquema de inversión de flujo es que en tales configuraciones se fuerza la formación de una onda térmica, pero sin invertir el sentido de circulación del fluido. Las primeras menciones a estos esquemas figuran en Matros (1989) y solo uno de los esquemas ha sido estudiado en un conjunto reducido de trabajos. El mismo corresponde a la red de reactores (normalmente formada por 2 o 3 reactores), con variación periódica en la posición de la alimentación, como se muestra en las Figuras 2.3 y 2.4 .

A diferencia del sistema de inversión de flujo, en donde la región central del lecho es mantenida a un nivel térmico elevado evitando que la onda térmica penetre excesivamente en el equipo, en este sistema se logra el movimiento de la onda térmica de un reactor a otro en forma cíclica, como resultado de la variación periódica de la posición de la alimentación.

Se ha demostrado que la red de reactores es técnicamente factible (e.g., $\mathrm{Ba}$ rresi y col., 1999; Brinkmann y col., 1999; Haynes y Caram, 1994). Sin embargo, el sistema resulta sensiblemente más inestable, especialmente si se 
lo compara con el reactor de inversión de flujo. En consecuencia, el rango de tiempos de ciclos que permiten una operación estable resulta muy estrecho (Madai y Sheintuch, 2008; Barresi y col., 2007; Fissore y Barresi, 2002).

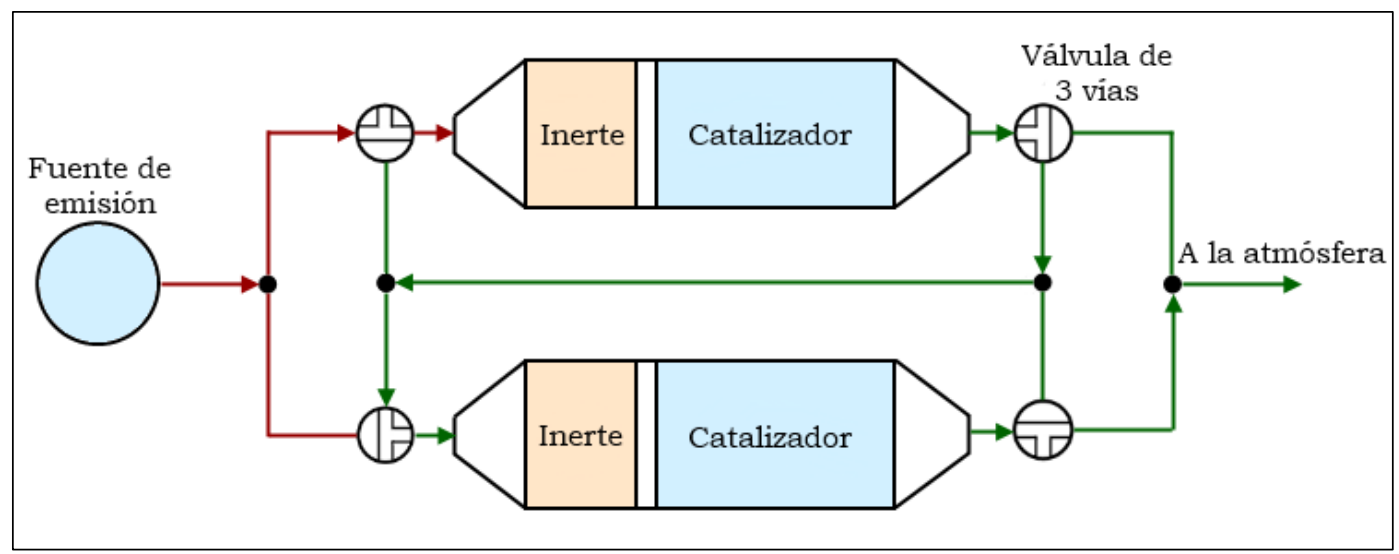

Figura 2.3: red de reactores constituida por 2 lechos.

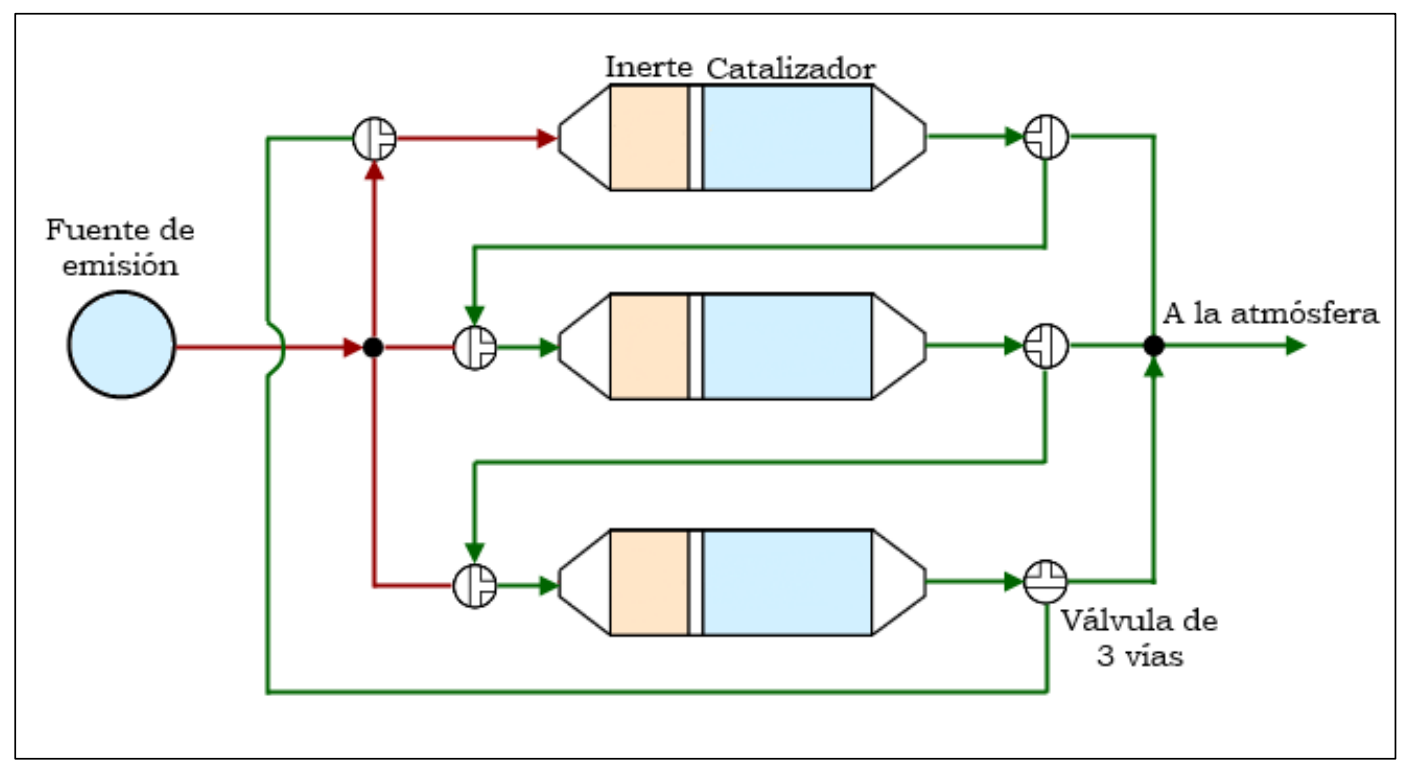

Figura 2.4: red de reactores constituida por 3 lechos.

\subsubsection{Biofiltración}

El proceso de biofiltración (Figura 2.5) es una técnica basada en la habilidad de los microorganismos (normalmente bacterias) para convertir contaminantes orgánicos en $\mathrm{H}_{2} \mathrm{O}, \mathrm{CO}_{2}$ y biomasa, bajo condiciones aeróbicas (en presencia de oxígeno). Si bien fue desarrollado originalmente con el objeti- 
vo de disminuir el olor de gases residuales, ha probado ser un método de remoción de VOCs efectivo y económicamente accesible.

El componente clave de la biofiltración es el biofiltro, el cual consiste en un lecho relleno de materiales naturales como compost, tierra, etc., rodeados de una microflora inmovilizada. El biofiltro debe mantenerse a una humedad elevada. Por ello, se trata de conservar el mismo mojado mediante el uso de dispersores que proveen agua continuamente al gas a tratar en un humidificador. El nivel de humedad es importante para el funcionamiento adecuado y la eficiencia del biofiltro, debido a que el proceso de degradación es exotérmico y tiende a secar los lechos filtrantes.

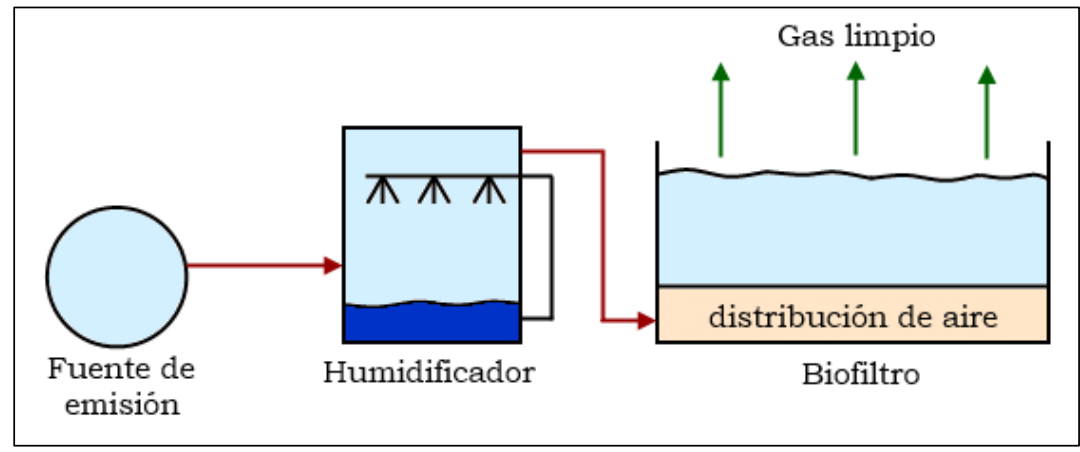

Figura 2.5: sistema de biofiltración.

Los contaminantes orgánicos en la corriente de aire a tratar se deberán disolver en agua para luego ser convertidos por los microorganismos en una biopelícula. El tipo de microorganismo depende del tipo de VOC a eliminar. Muchos microorganismos requieren condiciones de $\mathrm{pH}$ neutras.

Los materiales naturales utilizados como sustrato filtrante brindan los nutrientes necesarios para soportar el crecimiento y la supervivencia de los microorganismos. La descomposición de las células también lleva a la pérdida de nutrientes. El tamaño de la partícula sustrato es seleccionado de acuerdo al compromiso entre la superficie de absorción y la resistencia al flujo (Moretti, 2002).

El tipo más adecuado de biofiltro para emisiones de plantas químicas es un lecho fijo, donde la temperatura y la humedad pueden ser controladas de una manera efectiva y el monitoreo de los efluentes es sencillo. 
Componentes de bajo peso molecular que son solubles y contienen átomos de oxígeno son buenos candidatos para la biofiltración. Aldehídos, cetonas, alcoholes, éteres, ésteres y ácidos orgánicos se degradan rápidamente en este tipo de sistema (para compuestos fácilmente degradables se han alcanzado eficiencias de remoción superiores al 90\%). Sin embargo, la biofiltración no es efectiva frente a hidrocarburos halogenados y poliaromáticos (Moretti, 2002).

La degradación de compuestos con azufre, nitrógeno y halógenos llevan a la formación de ácidos que bajan el $\mathrm{pH}$ de las capas de biofiltros. En estos casos, se suman compuestos alcalinos al material filtrante para el control del $\mathrm{pH}$. Sin embargo, la acumulación de las sales formadas obstruye la circulación del lecho después de un periodo. La vida útil de los lechos oscila entre 2 y 5 años, aunque en algunos casos puede extenderse con lavados periódicos.

Los biofiltros no presentan los problemas energéticos de las oxidaciones térmicas (en mayor medida) o catalíticas (en menor medida), y pueden ser competitivos para bajas concentraciones de compuestos que sean fácilmente biodegradables. De todas maneras, pierden ventajas para altas cargas de contaminantes, particularmente si se trata de compuestos clorados (Leson y Winer, 1991).

Debido a la incertidumbre en el funcionamiento del proceso biológico, resultan indispensables las pruebas en escala piloto para la mayoría de las aplicaciones. Estas proveen un orden de magnitud para los parámetros que permiten estimar la eficiencia y el volumen del lecho filtrante necesario para lograr el nivel de destrucción de VOCs deseado.

\subsubsection{Fotocatálisis}

El proceso de fotocatálisis está basado en el empleo de luz ultravioleta (UV), la que posibilita que se lleven a cabo reacciones superficiales de oxidación de los VOCs a tratar, al iluminar un tipo específico de semiconductor (nanopartículas de $\mathrm{TiO}_{2}$ ). Este proceso ha recibido considerable atención en los últimos años, lo que puede justificar que no siempre se menciona en algunos trabajos de revisión (Everaert y col., 2002; Khan y Ghoshal, 2000) como una de las alternativas de tratamientos de VOCs para sistemas industriales. 
Por las características del sistema, una gran cantidad de trabajos orientan su aplicación a la purificación del aire en ambientes interiores, ya que tienen la gran ventaja de trabajar a temperatura ambiente, con baja pérdida de carga y llegando a límites muy bajos de concentración de VOCs. Esto los hace atractivos para su empleo en sistemas de ventilación, calefacción y acondicionamiento de aire, tanto nuevos como existentes (Jacoby y col., 1996). Se ha estudiado su aplicación en la eliminación de diversos tipos de VOCs, tanto de un único compuesto (Jacoby y col., 1996), como de mezclas de los mismos (Hodgson y col., 2007), encontrándose que una fracción significativa de los estudios se ha direccionado a la eliminación de formaldehído (Passalia y col., 2012), un compuesto que puede ser dificil de eliminar con otros métodos.

El empleo de este proceso en escalas industriales, donde deben tratarse grandes caudales de aire, requiere estudios ulteriores, a efectos de evitar algunos de los inconvenientes que pueden presentar el cambio de escala, como ha sido señalado por Ray (2000).

\subsection{Técnicas de recuperación de VOCs}

El objetivo de este tipo de técnicas es remover los VOCs presentes en la corriente de aire a tratar y recuperarlos para su posterior utilización. De esta forma, aparece un doble objetivo: cumplir con las normas ambientales que limitan la emisión de VOCs y alcanzar una recuperación y reutilización de los mismos. Para lograr esto existen distintas tecnologias, basadas en procesos de adsorción, absorción, condensación o mediante el empleo de membranas.

\subsubsection{Adsorción}

En el control de la contaminación del aire, la adsorción se emplea para remover VOCs de corrientes de gas con baja o media concentración, cuando debe alcanzarse alguna concentración restringida de salida o se desea la recuperación del VOC.

El diseño de un sistema de adsorción depende de las características del VOC que vaya a ser recuperado, de las condiciones operativas de la corriente de gas a la entrada de la columna (temperatura, presión, caudal volumétrico) y 
las propiedades físicas del adsorbente empleado. Los sistemas de adsorción típicos empleados en la industria utilizan carbón activado, zeolita o adsorbentes poliméricos.

La corriente de proceso circula a través del adsorbedor, donde los VOCs resultan ser selectivamente retenidos por el material adsorbente hasta que la presencia de los mismos se evidencia en el efluente. Alcanzada una cierta concentración máxima permitida a la salida del adsorbedor, se debe discontinuar la operación para que el mismo pueda ser regenerado. Se pueden seguir varias alternativas para lograr este objetivo (Ghoshal y Manjare, 2002); los procedimientos más utilizados se basan en la modificación de las condiciones de temperatura (Temperature Swing Adsorption, TSA) o presión (Pressure Swing Adsorption, $P S A)$. El proceso de regeneración puede ser repetido varias veces; sin embargo, el material adsorbente debe ser reemplazado eventualmente debido a un descenso gradual de su capacidad de adsorción. Los vapores obtenidos durante la desorción son llevados a un sistema de recuperación, como un condensador-decantador o torre de destilación.

Un esquema típico que permite la operación continua, de acuerdo al esquema mencionado en el párrafo anterior, corresponde a un sistema de lechos en paralelo que operan en forma alternada (Figura 2.6). Mientras uno de los lechos está trabajando como adsorbedor, el otro está en espera o en proceso de regeneración. En forma similar a los sistemas de intercambio regenerativo, es posible utilizar como variante al sistema de dos lechos en paralelos un adsorbedor rotativo.

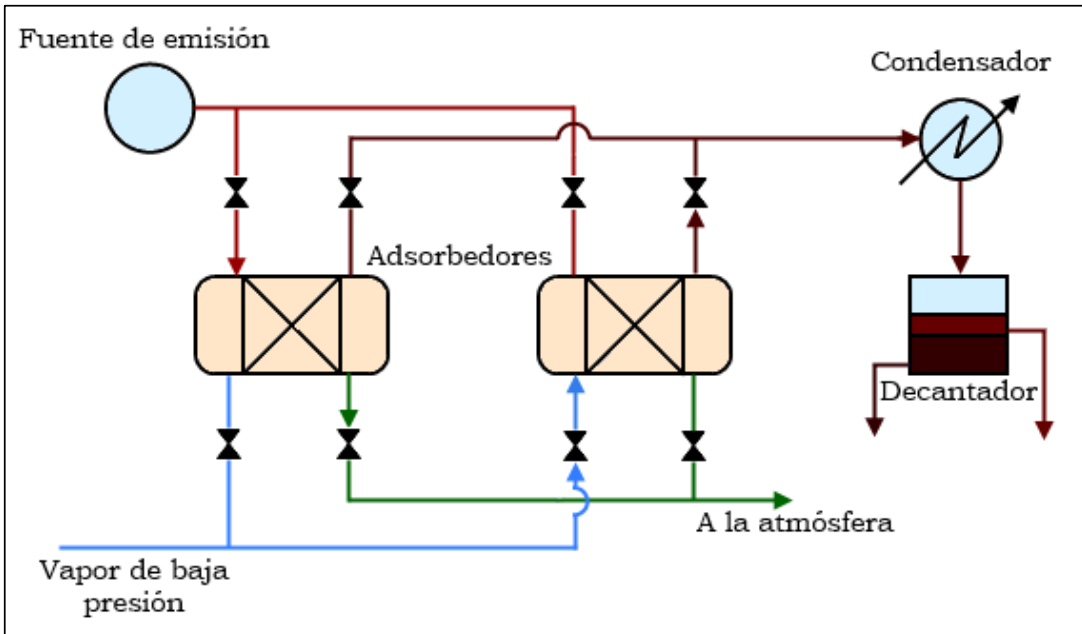

Figura 2.6: sistema de adsorción. 
Para este tipo de sistemas se alcanzan eficiencias de remoción superiores a un 95\% (Moretti, 2002). La adsorción es aplicable a corrientes continuas e intermitentes y tiene la capacidad de manejar un amplio rango de concentraciones. Si bien los sistemas de adsorción resultan aptos para la recuperación de una gran variedad de VOCs, en ciertas circunstancias su empleo no es recomendado. Particularmente:

* Para la adsorción de altas concentraciones de VOCs puede producirse un excesivo aumento de temperatura en el lecho como consecuencia de la energía liberada en la adsorción. No obstante, debe aclararse que en presencia de vapores inflamables, los requerimientos de seguridad limitan las concentraciones de los VOCs a la entrada a un 25\% del LEL (Moretti, 2002)

* Los compuestos de peso moleculares muy elevados (>130 kg/kmol) que son caracterizados por baja volatilidad (punto de ebullición mayor a $200{ }^{\circ} \mathrm{C}$ ), son fuertemente adsorbidos, haciendo dificil su desorción durante la regeneración. Por otro lado, los compuestos de bajo peso molecular no presentan una adecuada adsorción en adsorbentes típicos, como el carbón activado.

* Los sistemas de adsorción operados de manera apropiada pueden ser muy efectivos cuando se manejan corrientes que contiene una mezcla de componentes de características similares, pero no cuando la mezcla corresponde a compuestos de bajo y alto peso molecular simultáneamente. Los componentes orgánicos más livianos tienden a ser desplazados de la superficie adsorbente por los más pesados, reduciendo considerablemente la eficiencia del sistema.

* Finalmente, los niveles de humedad de las corrientes de gas que superen el $50 \%$ pueden afectar la capacidad del adsorbente a concentraciones menores de 1000 ppm de adsorbato. La humedad relativa puede reducirse agregando aire seco a la corriente o calentando el gas en un intercambiador de calor. 


\subsubsection{Absorción}

Esta alternativa consiste en remover los VOCs contenidos en una corriente gaseosa poniéndola en contacto con un solvente líquido. Todos los VOCs solubles resultan transferidos a la fase líquida y de esta manera se logra limpiar la corriente gaseosa.

Este tipo de separación se lleva a cabo por medio de una torre de absorción diseñada para proveer el área necesaria de contacto líquido-vapor y así facilitar la transferencia de masa.

La absorción es generalmente utilizada para limpiar corrientes gaseosas con altas concentraciones de componentes orgánicos, especialmente aquellos solubles en agua, como metanol, etanol, isopropanol, butanol, acetona y formaldehído.

Existen algunos factores que limitan su uso:

* disponibilidad de un solvente adecuado, ya que los VOCs deben ser solubles en el líquido absorbente (e.g., agua, aceites minerales);

* disponibilidad de información del equilibrio líquido-vapor para sistemas específicos componente orgánico-solventes en cuestión, necesaria para diseñar el sistema absorbedor. En muchas ocasiones no está disponible para compuestos orgánicos no comunes.

Otro aspecto a tener en cuenta para este tipo de sistemas es el tratamiento del solvente luego del proceso de extracción. En muchos casos el líquido que se utilizó para absorber los VOCs es recuperado por desorción de los mismos, típicamente por elevación de temperatura o por vacío, y los VOCs son recuperados como un líquido en un condensador posterior.

\subsubsection{Condensación}

La separación por condensación puede ser llevada a cabo incrementando la presión del sistema a una dada temperatura (condensación por compresión), disminuyendo la temperatura a una presión constante (condensación por refrigeración) o ambas. 
En un sistema de dos componentes donde uno de ellos es no-condensable (aire), la condensación ocurre cuando la presión parcial del componente volátil iguala a la presión de vapor (punto de saturación). Para los compuestos más volátiles, una gran cantidad de los mismos se mantiene como vapor a una dada temperatura. Por lo tanto, para remover o recuperar el compuesto, se requiere una temperatura más baja para producir la saturación y condensación. En estos casos, se utiliza la refrigeración para obtener las temperaturas bajas necesarias para lograr las eficiencias de remoción requeridas.

Esta técnica es más eficiente para VOCs con puntos de ebullición por encima de los $40^{\circ} \mathrm{C}$ a relativamente altas concentraciones (>5000 ppm), alcanzándose eficiencias que varian usualmente desde 50 a 90\% (Everaert y col., 2002). Para la obtención de temperaturas muy bajas puede utilizarse nitrógeno líquido, pero esto aumenta fuertemente los costos operativos (Khan y Ghoshal, 2000).

El equipamiento básico de un sistema de condensación refrigerado incluye esencialmente un condensador y una unidad de refrigeración. Los condensadores pueden ser usados para remover VOCs no halogenados y halogenados, sin necesidad de equipamiento auxiliar costoso. Si la corriente de venteo contiene vapor de agua o si el VOC tiene un alto punto de congelación, puede formarse hielo o hidrocarburos congelados en los tubos o placas del condensador. Esto reduce la eficiencia en la transferencia de energía y aumenta la pérdida de carga en el condensador. En estos casos puede ser usado un pre-enfriador para remover la humedad antes de que la corriente ingrese al condensador.

\subsubsection{Membrana}

En un separador de membrana típico, la corriente de gas a tratar es alimentada a un arreglo de módulos de membranas, preferentemente permeables a los componentes orgánicos. Los VOCs en la corriente permeada son luego condensados y removidos como un líquido para su recuperación, mientras que el gas purificado es removido como un residuo.

El paso a través de la membrana es inducido por una diferencia de presión a ambos lados de la misma. El compuesto atraviesa la membrana a una de- 
terminada velocidad, dependiendo de la permeabilidad del material y de la fuerza impulsora.

Los sistemas de separación por membranas pueden tener uno o múltiples pasos, tantos como sean necesarios para lograr las eficiencias de recuperación deseadas. La vida útil de las membranas es, en promedio, de tres años.

Existen diversos factores que limitan la aplicabilidad de los sistemas de membrana para la recuperación de VOCs (Khan y Ghoshal, 2000):

* adecuación al tratamiento de un conjunto de compuestos orgánicos,

* costo de la membrana,

* mantenimiento de la membrana,

* velocidad de permeación,

* reutilización de la membrana,

* humedad de la corriente.

\subsection{Caracteristicas del sistema a tratar; fundamentación de la selección del conjunto de técnicas}

En términos generales, un criterio adecuado para la selección de al menos un primer grupo de técnicas de tratamiento, sería elegir la técnica y el esquema que permitan alcanzar el objetivo (nivel de VOCs requerido), con el menor costo global posible. Aunque para cada caso específico se puedan definir criterios que permitan retener ciertas metodologias y desechar otras, una primera selección se apoya normalmente en: la concentración de VOCs, el caudal de la corriente gaseosa y el nivel de control requerido. Entre estas, el caudal de aire y la concentración de VOCs en la corriente son los dos aspectos más relevantes para la selección del método de tratamiento. Muchas de las alternativas descritas en las Secciones 2.1 y 2.2, o bien presentan impedimentos de tipo técnico para ser llevadas a cabo fuera de ciertos rangos o dejan de ser económicamente convenientes.

Existen en bibliografia diagramas que permiten seleccionar un conjunto de tecnologías que podrian ser aptas para cada problema (Moretti, 2002; Shah y 
col., 2000). Los mismos son normalmente de doble entrada, debiendo ingresar con el caudal de aire y la concentración de VOCs a tratar, como se verá en la Sección 2.3.2.

\subsubsection{Caracteristicas de la corriente a tratar}

La corriente a tratar considerada para el análisis y diseño de los sistemas de eliminación en este trabajo (Tabla 2.1), corresponde a una corriente de aire proveniente de una planta de impresión de envases. La misma está contaminada con acetato de etilo y etanol, como consecuencia de la ventilación del recinto donde se lleva a cabo el proceso de impresión.

Tabla 2.1: características de la corriente a tratar

\begin{tabular}{cccccc}
$\begin{array}{c}\text { Tipo de } \\
\text { emisión }\end{array}$ & $\begin{array}{c}\text { Caudal } \\
{\left[\mathbf{N m}^{\mathbf{3}} / \mathbf{s}\right]}\end{array}$ & $\begin{array}{c}\text { Presión } \\
{[\mathbf{b a r}]}\end{array}$ & $\begin{array}{c}\text { Temperatura } \\
{\left[{ }^{\circ} \mathbf{C}\right]}\end{array}$ & $\begin{array}{c}\text { Composición (nominal) } \\
\text { [ppm] }\end{array}$ \\
\hline \multirow{2}{*}{ permanente } & 9.86 & 1.01 & 50 & Acetato de etilo & Etanol \\
\cline { 3 - 5 } & & & & 615 & 250 \\
\hline
\end{tabular}

Las características informadas en la Tabla 2.1 corresponden al contenido de VOCs nominal. Sin embargo, a diferencia de un proceso de producción, es usual que las condiciones de la corriente a tratar fluctúen de manera marcada, lo que deberá tenerse en cuenta en el diseño del proceso. Más aún, si se considera que el caudal será fijo (la ventilación del ambiente de trabajo puede realizarse por medio de un soplador que mantenga el caudal de aire), de las restantes fluctuaciones posibles, el contenido total de VOCs es la que posee mayor impacto en el diseño del sistema de tratamiento, como se verá en la discusión de los resultados alcanzados. Teniendo presente esto, se considerará para el diseño de cada uno de los sistemas un intervalo de contenido de VOCs, correspondiendo el máximo al informado en la Tabla 2.1 (nominal) y el mínimo a la mitad de dicho valor. En términos de la máxima elevación adiabática, $\Delta T_{a d}$, dichos extremos corresponden a 53.2 y $26.6^{\circ} \mathrm{C}$, respectivamente.

Tanto el acetato de etilo como el etanol son altamente inflamables y la exposición a los mismos puede traer severas consecuencias a corto y largo plazo para la salud humana y el medio ambiente. Por esta razón, es necesario dis- 
minuir su concentración a niveles que puedan considerarse inocuos. Con esta finalidad, se tomaron como referencia los estándares internacionales. Siguiendo los niveles de concentración recomendados por la Comisión Europea (Directiva 2010/75, 2010), se asumió para los cálculos una tolerancia de $20 \mathrm{mgC} / \mathrm{Nm}^{3}$ de VOCs totales en la corriente descargada a la atmósfera ( 9 ppm si se considerase solo al acetato de etilo), correspondiendo este valor a un promedio temporal.

\subsubsection{Verificación de la selección de la técnica}

Debido a que se plantea como objetivo específico la eliminación de los vapores de acetato de etilo y etanol y no la recuperación de los mismos, el análisis quedará centrado en las técnicas de eliminación de VOCs. Cabe aclarar que esto no implica que ninguna de las técnicas de recuperación resulte factible para el presente caso de estudio.

Teniendo en cuenta el nivel de concentración y el caudal a tratar, informados en la Tabla 2.1, es posible realizar un análisis de las distintas alternativas de tratamiento de efluentes desde un punto de vista económico. Como fuera mencionado previamente, existen gráficos construidos en función de la experiencia recogida de las unidades en funcionamiento. Uno de estos gráficos se muestra en la Figura 2.7 (Shah y col., 2000), donde se ha adicionado una marca señalando las condiciones nominales del caso a tratar (punto "A").

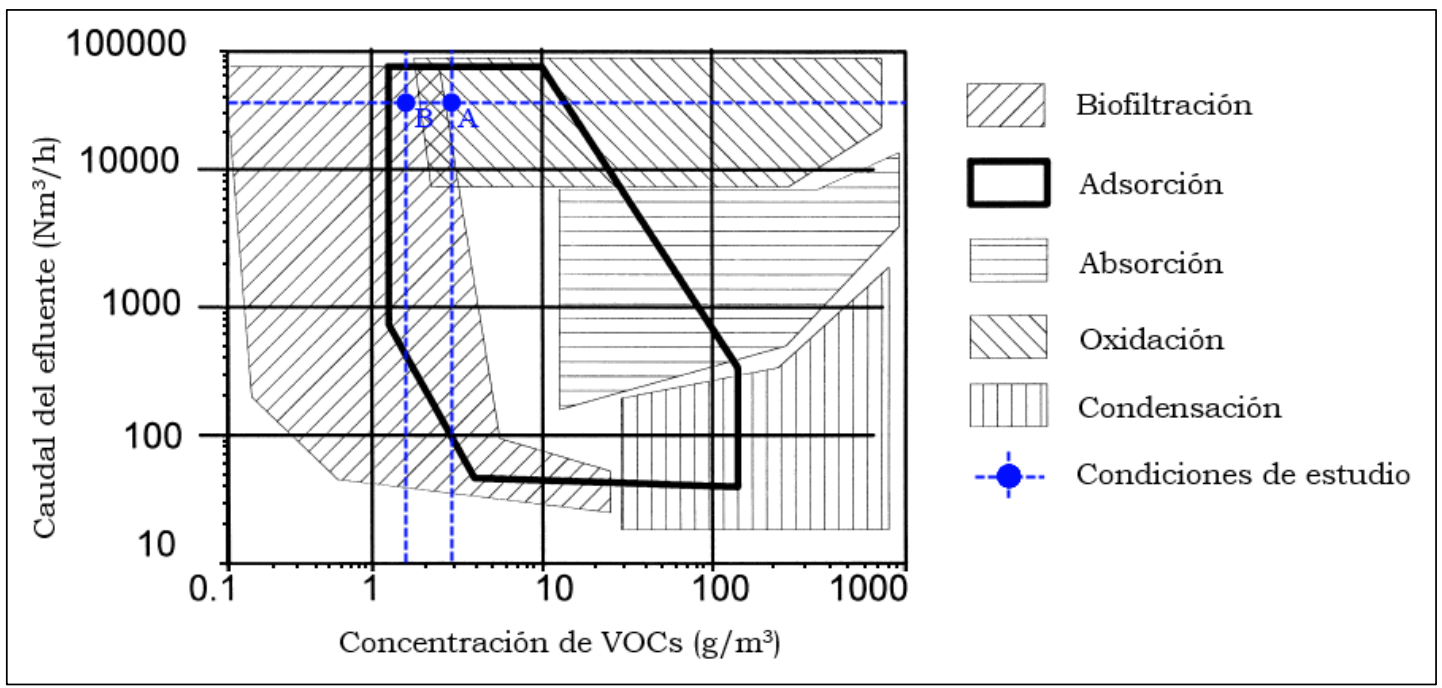

Figura 2.7: rango de condiciones operativas para distintas técnicas de tratamiento de VOCs. 
De dicha figura, se desprende que la oxidación de los $\mathrm{VOCs}$ a $\mathrm{CO}_{2}$ y $\mathrm{H}_{2} \mathrm{O}$ surge como la opción más adecuada. Adicionalmente, se señaló el punto correspondiente a la condición de mínimo contenido de VOCs tolerable. Si bien este punto queda fuera de la región donde la oxidación es considerada económicamente ventajosa, se puede apreciar que se encuentra muy cerca del límite de la misma.

\subsubsection{Selección entre oxidación térmica y catalitica y es- quemas de intercambio}

Habiendo escogido la oxidación como técnica más conveniente, resta discriminar si la misma será térmica o catalítica y el esquema a utilizar para el aprovechamiento de la energía liberada por la reacción (precalentamiento de la corriente a tratar).

La opción más elemental corresponde a la incineración térmica sin precalentamiento. Si bien esta opción puede disminuir los costos fijos, dicha reducción se verá severamente descompensada por el aumento en los costos operativos como consecuencia del uso de combustible adicional. De acuerdo a la descripción desarrollada en la Sección 2.1.1, otra alternativa corresponde a la incineración térmica recuperativa. Esta opción permite disminuir fuertemente el uso de combustible adicional pero se requiere de intercambiadores muy voluminosos como consecuencia del elevado caudal a tratar y la baja fuerza impulsora para la transferencia de energia $\left(\Delta T_{a d}\right)$. Debido a esto, ambas opciones se descartan como alternativas de tratamiento para el presente caso.

La otra alternativa correspondiente a la oxidación térmica que resta evaluar es la incineración térmica regenerativa (por inversión de flujo o utilizando intercambiadores rotativos). Si bien existen pequeñas diferencias entre ambos, se pueden generalizar los resultados encontrados por Gosiewski y Pawlaczyk (2014). En dicho trabajo, se concluye que el reactor térmico de inversión de flujo (RTIF) resultará más adecuado que el catalítico (RCIF) cuando la corriente a tratar posea un contenido de VOCs tal que la máxima elevación adiabática supere los $100^{\circ} \mathrm{C}$. En ese caso, será económicamente viable obtener vapor de alta presión a partir de la energía liberada por la reacción, situación que se ve favorecida por los mayores niveles térmicos. Sin embargo, cuando el contenido 
de VOCs a tratar resulte menor, la posibilidad de recuperar energía como vapor ya no será atractiva. Bajo tales condiciones el RCIF resulta al menos competitivo frente al RTIF. Si el contenido de VOCs disminuye aún más, el RCIF puede incluso posicionarse como una mejor alternativa, debido a que permite mantener la autotermia sin utilizar combustible adicional para contenidos de VOCs que superen los $15^{\circ} \mathrm{C}$ de $\Delta T_{a d}$, mientras que el RTIF necesita un $\Delta T_{a d}$ entre 45 a $70^{\circ} \mathrm{C}$ (Matros y col., 1996).

De acuerdo a las características de la corriente a tratar informadas en la Tabla 2.1 y considerando la discusión previa, se puede descartar también la incineración térmica regenerativa. Esto reduce la búsqueda de esquemas a analizar únicamente a aquellos donde la combustión se lleva a cabo en presencia de un catalizador sólido.

De las alternativas de combustión catalítica analizadas en la Sección 2.1.1, el esquema autotérmico utilizando intercambiadores recuperativos ya ha sido analizado previamente en el grupo de trabajo (Campesi y col., 2007). Por otro lado, la red de reactores no parece ser una opción adecuada, debido a que el rango de condiciones operativas en el cual resulta estable es notoriamente menor que en los sistemas convencionales o de inversión de flujo. Luego, los esquemas autotérmicos de combustión catalítica restantes son:

* reactor de inversión de flujo con inversión mediante válvulas;

* reactor de inversión de flujo con inversión mediante la rotación sobre un eje;

* reactor catalítico estacionario con precalentamiento de la corriente de aire mediante un intercambiador rotativo (lo llamaremos esquema regenerador-reactor, para simplificar).

Cada uno de estos esquemas poseen una característica común: el intercambio de energía es de tipo regenerativo y por ende el estado de operación se caracteriza por ser un estado transiente, pero cíclicamente estacionario (css), como fuera explicado previamente. 
- 32 - 


\section{Capítulo 03}

\section{- Modelado}

- Descripción de los sistemas a estudiar

- Sistema reactivo

- Modelo para el sistema de inversión de flujo

- Modelo para el sistema regenerador-reactor

- Aproximación de los sistemas de inversión de flujo para bajos tiempos de ciclo: reactor en contra-corriente 

En este capítulo se presentan los esquemas del sistema de inversión de flujo rotativo y del sistema regenerador-reactor, descritos brevemente en el Capítulo 2, analizando las diferencias básicas de cada uno.

En segundo lugar, se describe el sistema reactivo y las correspondientes expresiones cinéticas de las reacciones involucradas. Se presentan los modelos a utilizar para la simulación de cada esquema de tratamiento seleccionado, discutiendo las principales simplificaciones realizadas en cada caso. Adicionalmente, se presenta una aproximación para la operación con inversión de flujo a tiempos de ciclos muy pequeños.

Finalmente, se resumen las correlaciones y fórmulas utilizadas para el cálculo de las propiedades físicas de la corriente, de los coeficientes de transferencia de materia y energía y de los parámetros del modelo (Apéndice $3.1 \mathrm{y}$ 3.2). En adición, se presenta en el Apéndice 3.3 una formulación que permite visualizar el comportamiento del regenerador a bajos tiempos de ciclo, de forma similar a un intercambiador en contra-corriente.

\subsection{Descripción de los esquemas a estudiar}

En el Capítulo 2 se redujeron los esquemas a analizar en el presente estudio a tres tipos: los sistemas de inversión de flujo (considerando dos variantes, el esquema convencional y el rotativo) y el sistema regenerador-reactor. En dicho capítulo se mostró un esquema general para la operación con inversión de flujo convencional (Figura 2.2) y se mencionó la alternativa de montar el esquema sobre un sistema rotativo, sin ahondar en mayores detalles sobre las diferencias entre una u otra opción, más allá de los criterios que permiten establecer los tiempos mínimos admisibles.

Teniendo presente la descripción de la operación con inversión de flujo realizada en el Capítulo 2, se puede visualizar que cuanto mayor sea el tiempo de duración del ciclo, más va a penetrar la onda térmica en el sistema. En estas circunstancias, partiendo de condiciones de operación y diseños definidos, si se operase el equipo con tiempos de ciclos significativamente mayores que el estipulado, el sistema se apagaría a menos que el volumen del lecho se aumentase. En base a este razonamiento, se puede concluir que cuanto menor sea el tiempo de ciclo, más pequeño podrá ser el equipo. Sin embargo, existen 
dos factores que limitan el tiempo de ciclo mínimo que puede ser utilizado en la práctica. En primer lugar, las válvulas poseen una vida útil que dependerá de la cantidad de veces que la misma deba modificar su posición. De acuerdo con Barresi y col. (2007), las válvulas utilizadas en estos sistemas soportan unos 500000 movimientos. Con ello, y considerando que por cada ciclo la válvula requerirá dos movimientos, si se pretende que la vida útil de la misma alcance al menos dos años, el tiempo de ciclo no deberá ser menor que $240 \mathrm{~s}$. Lógicamente, si se operase con tiempos de ciclos aún menores, se reduciría la vida útil de las válvulas. De todas maneras, aun cuando fuese aceptable disminuir más el tiempo de ciclo, esto no será siempre recomendable pues comenzará a ser significativo otro problema: los VOCs remanentes en la región inerte y en la porción de la región catalítica que se hubiera enfriado (así como también la cantidad remanente en las tuberías), son expulsados sin haber reaccionado cada vez que el sentido de circulación de la corriente se invierte. Cuanto menor sea el tiempo de ciclo, más importante será la contribución de dicha emisión en el valor global, pudiendo inclusive superar los niveles de tolerancia estipulados para tiempos muy pequeños, solo por este efecto.

La última problemática se puede solucionar utilizando tres lechos en paralelo, como se describe en Kolios y col. (2000). Mientras la corriente a tratar circula en una dirección dada en uno de los lechos, el efluente del mismo transfiere su energía a otro de ellos, circulando en sentido opuesto a la dirección de alimentación y el tercer lecho se limpia haciendo circular aire puro en el mismo sentido de alimentación de la corriente a tratar. El inconveniente de este esquema es que siguen siendo necesarias las válvulas (de hecho serán necesarias un mayor número de ellas), con lo cual se mantiene el problema de la máxima vida útil.

Una alternativa que ha sido poco explorada y permite solucionar ambos problemas es la utilización de un lecho rotativo, como fuera descrito en el Capítulo 2, lo que motivó su análisis en el presente estudio.

Tomando como referencia la velocidad de rotación de los intercambiadores rotativos (de 2 a $10 \mathrm{rpm}$ típicamente), se puede concluir que estos esquemas podrán operar, en principio, para tiempos de ciclos de hasta $6 \mathrm{~s} \mathrm{(10} \mathrm{rpm).} \mathrm{Adi-}$ cionalmente, este esquema permite utilizar una pequeña porción de la sección total de flujo, para el barrido del contenido residual de VOCs en los canales 
inmediatos a la posición donde se produce la inversión de flujo. De esta manera, el sistema queda dividido en dos tipos de regiones, la región destinada al tratamiento de la corriente (región o etapa de reacción) y la región destinada a la limpieza del canal (región o etapa de limpieza). En la Figura 3.1 se muestra un diagrama de este esquema, donde se han marcado las partes básicas de separación de cada corriente (en color gris) para facilitar la interpretación.

En principio, la desventaja de este esquema comparado con el convencional, radica en su mayor grado de complejidad. Una forma de simplificar levemente el esquema presentado sería realizando el barrido de los VOCs remantes directamente con aire del ambiente. Sin embargo, esto requeriría de la instalación de un soplador adicional, situación que prefirió evitarse.

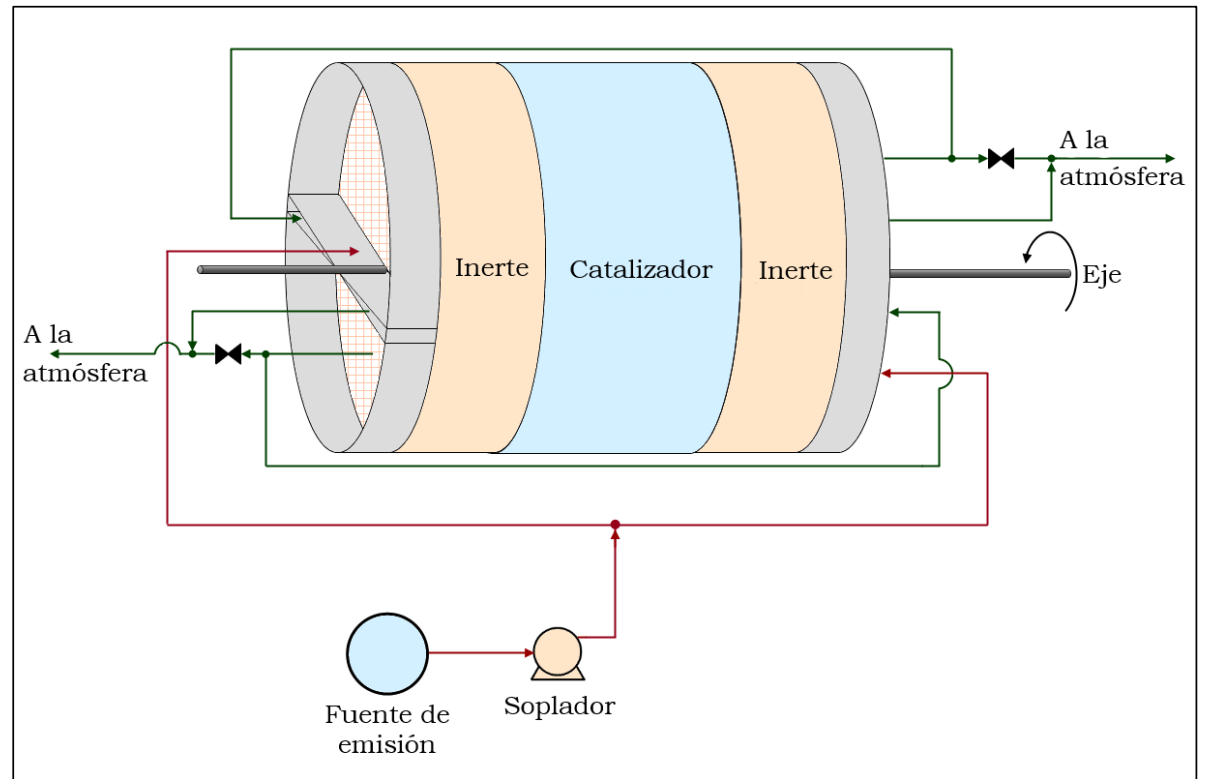

Figura 3.1: esquema del sistema de inversión de flujo rotativo.

En cuanto a la relación entre los tiempos de duración de cada etapa respecto del tiempo de ciclo, definiendo la fracción de la sección total destinada para la limpieza como $f_{L}$, se puede ver fácilmente que:

$$
\begin{aligned}
& t_{E R}=\left(1-f_{L}\right) t_{\text {ciclo }} / 2 \\
& t_{E L}=f_{L} t_{\text {ciclo }} / 2
\end{aligned}
$$


siendo $t_{E R}$ el tiempo de duración de la etapa de reacción, $t_{E L}$ el tiempo de duración de la etapa de limpieza y $t_{c i c l o}$ el tiempo total de un ciclo (relacionado con la velocidad de rotación, $\omega$, por la relación $\left.t_{\text {ciclo }}=\omega^{-1}\right)$.

Por otro lado, el sistema regenerador-reactor presenta en su concepción algunas características similares al sistema de inversión de flujo descrito en los párrafos precedentes. Como se aprecia en la Figura 3.2, en el regenerador se pueden distinguir tres regiones: la primera donde la corriente a tratar se calienta a expensas del enfriamiento del sólido (región o etapa de enfriamiento), la segunda destinada al barrido del contenido residual de VOCs en los canales inmediatos a la posición donde ingresa la corriente efluente del reactor y la última donde dicha corriente cede su energía al lecho regenerando (calentando) así al sólido (región o etapa de calentamiento). A fin de evitar confusiones, cabe resaltar que la denominación de las etapas de enfriamiento o calentamiento hacen referencia a lo que ocurre con el sólido en el equipo, verificándose naturalmente lo opuesto para la corriente gaseosa.

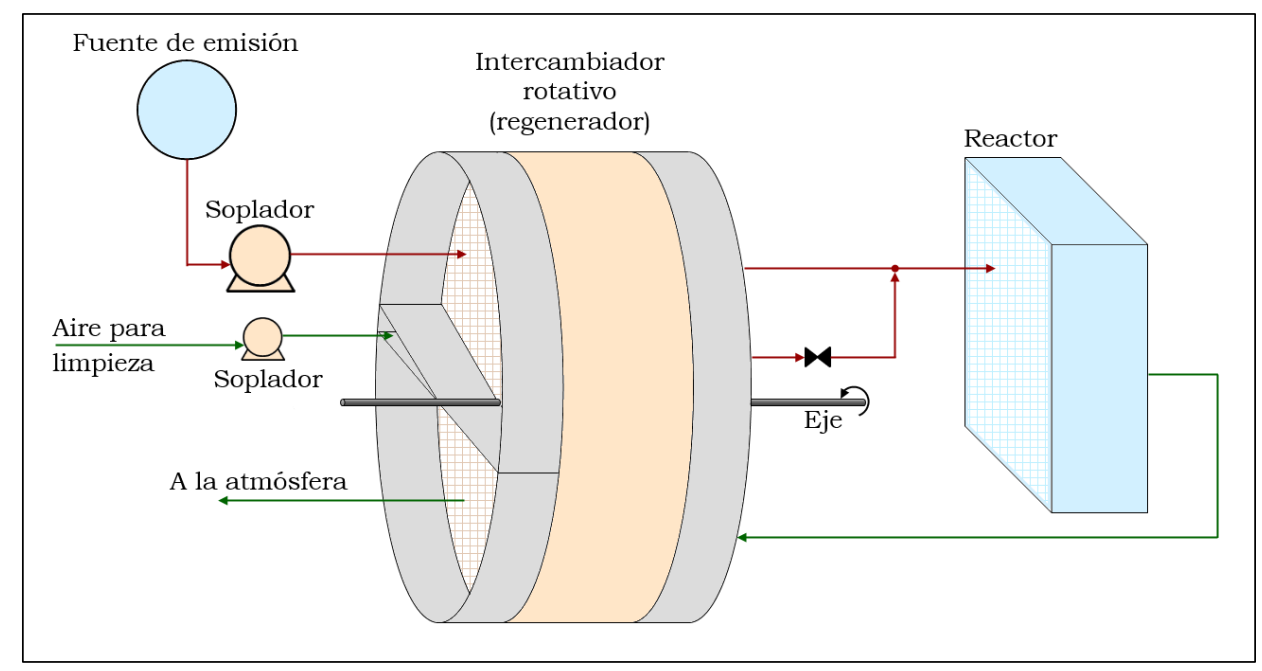

Figura 3.2: esquema del sistema regenerador-reactor.

En cuanto a la relación entre los tiempos de duración de cada etapa respecto del tiempo de ciclo, definiendo las fracciones de la sección total destinada para la región de enfriamiento y de limpieza como $f_{E}$ y $f_{L}$, respectivamente, se puede ver fácilmente que los tiempos de duración de cada etapa serán:

$$
t_{E E}=f_{E} t_{\text {ciclo }}
$$




$$
\begin{aligned}
& t_{E L}=f_{L} t_{\text {ciclo }} \\
& t_{E C}=\left(1-f_{E}-f_{L}\right) t_{\text {ciclo }}
\end{aligned}
$$

siendo $t_{E E}$ el tiempo de duración de la etapa de enfriamiento, $t_{E L}$ el tiempo de duración de la etapa de limpieza, $t_{E C}$ el tiempo de duración de la etapa de calentamiento y $t_{c i c l o}$ el tiempo total de un ciclo (relacionado al igual que en el sistema de inversión de flujo con la velocidad de rotación, $\omega$, por la relación $\left.t_{\text {ciclo }}=\omega^{-1}\right)$.

\subsection{Sistema reactivo}

Para el presente estudio, se consideró un catalizador en base a $\mathrm{Mn}$ y $\mathrm{Cu}$ como agentes catalíticos, en una relación molar de 9:1 respectivamente $\left(\mathrm{Mn}_{9} \mathrm{Cu}_{1}\right)$. El mismo fue desarrollado por un grupo de trabajo perteneciente al INTEQUI (CONICET/UNSL), siendo razonablemente económico y mostrando una buena actividad a partir de temperaturas cercanas a los $200{ }^{\circ} \mathrm{C}$ (Morales $y$ col., 2008). Las características del catalizador se informan en la Tabla 3.1.

Tabla 3.1: propiedades del catalizador.

\begin{tabular}{ccccc}
\hline $\begin{array}{c}\boldsymbol{d}_{\text {poro }} \\
{[\mathbf{m}]}\end{array}$ & $\varepsilon_{\mathrm{c}}$ & $\tau_{\mathrm{c}}$ & $\begin{array}{c}\rho_{\mathrm{c}} \\
{\left[\mathbf{k g} / \mathbf{m}^{\mathbf{3}}\right]}\end{array}$ & $\begin{array}{c}\boldsymbol{c}_{\boldsymbol{p c}} \\
{[\mathbf{J} /(\mathbf{k g ~ K})]}\end{array}$ \\
\hline $12.510^{-9}$ & 0.41 & 1.6 & 2745 & 836 \\
\hline
\end{tabular}

siendo:

$d_{\text {poro }}:$ diámetro medio de poro,

$\varepsilon_{C}:$ porosidad del catalizador

$\tau_{C}:$ tortuosidad del catalizador

$\rho_{C}:$ densidad del catalizador

$c_{p C}:$ calor específico a presión constante del catalizador. 
Dicho catalizador se supone impregnado sobre una matriz de cordierita no porosa (monolito) con canales cuadrados, cuyas características se muestran en la Tabla 3.2 (Gulati, 2006). Este material no solo se considera como soporte del catalizador sino que también constituye el material elegido como inerte. Para todos los casos, el espesor del depósito considerado fue de $10 \mu \mathrm{m}$.

Tabla 3.2: propiedades de la cordierita.

\begin{tabular}{ccc}
\hline $\begin{array}{c}\rho_{\text {cor }} \\
{\left[\mathbf{k g} / \mathbf{m}^{3}\right]}\end{array}$ & $\begin{array}{c}\boldsymbol{c}_{\text {p,cor }} \\
{[\mathbf{J} / \mathbf{( k g ~ K )})}\end{array}$ & $\begin{array}{c}\lambda_{\text {cor }} \\
{[\mathbf{J} /(\mathbf{m ~ s ~ K})]}\end{array}$ \\
\hline 2511 & 1046 & 0.42 \\
\hline
\end{tabular}

siendo:

$\rho_{c o r}:$ la densidad de la cordierita

$c_{p, c o r}:$ el calor específico a presión constante de la cordierita

$\lambda_{\text {cor }}:$ la conductividad térmica de la cordierita en el sentido axial.

Teniendo presente que el material inerte y el catalitico poseen propiedades térmicas ligeramente diferentes (la capacidad calorifica por unidad de volumen, $\rho c_{p}$, es $2.6310^{6}\left[\mathrm{~J} \mathrm{~m}^{-3} \mathrm{~K}^{-1}\right]$ para el inerte y $2.2910^{6}\left[\mathrm{~J} \mathrm{~m}^{-3} \mathrm{~K}^{-1}\right]$ para el catalizador), para la región catalítica de los equipos se consideró:

$$
\left(1-\varepsilon_{L, C}\right)\left(\rho c_{p}\right)_{S, C}=\left(1-\varepsilon_{L}^{0}\right) \rho_{c o r} c_{p, c o r}+\left(\varepsilon_{L}^{0}-\varepsilon_{L, C}\right) \rho_{C} c_{p C}
$$

donde $\left(\rho c_{p}\right)_{S, C}$ representa la capacidad calorífica del sólido por unidad de volumen de sólido para dicha región, $\varepsilon_{L}^{0}$ la porosidad que tendría el lecho sin el depósito catalítico y $\varepsilon_{L, C}$ la porosidad del lecho considerando el depósito.

Si bien la corriente a tratar contiene solo dos componentes, acetato de etilo $(A E)$ y etanol $(E t)$, se ha verificado la formación y acumulación de acetaldehído (Ac) como resultado de la oxidación parcial del etanol. De esta manera, el sis- 
tema reactivo queda conformado por la siguiente terna de reacciones (Campesi y col., 2012b):

$$
\begin{aligned}
& \mathrm{C}_{4} \mathrm{H}_{8} \mathrm{O}_{2}+5 \mathrm{O}_{2} \rightarrow 4 \mathrm{CO}_{2}+4 \mathrm{H}_{2} \mathrm{O} \\
& \mathrm{C}_{2} \mathrm{H}_{6} \mathrm{O}+1 / 2 \mathrm{O}_{2} \rightarrow \mathrm{C}_{2} \mathrm{H}_{4} \mathrm{O}+\mathrm{H}_{2} \mathrm{O} \\
& \mathrm{C}_{2} \mathrm{H}_{4} \mathrm{O}+\frac{5}{2} \mathrm{O}_{2} \rightarrow 2 \mathrm{CO}_{2}+2 \mathrm{H}_{2} \mathrm{O}
\end{aligned}
$$

Nombrando a los componentes claves de acuerdo con: 1 - acetato de etilo, 2 - etanol, 3 - acetaldehído, las expresiones cinéticas para cada una de las reacciones 1 a 3, expresiones del tipo LHHW con un término de inhibición común, se pueden escribir como:

$$
r_{i}=\frac{k_{i} C_{i}}{1+K_{1} C_{1}+K_{3} C_{3}} \quad \text { para } i=1 . .3
$$

siendo

$$
k_{i}=k_{i, r e f} \exp \left[-\frac{E_{i}}{R}\left(\frac{1}{T}-\frac{1}{T_{r e f, c i n}}\right)\right]
$$

\begin{tabular}{|c|c|c|c|c|c|}
\hline \multicolumn{2}{|c|}{$\begin{array}{c}\text { Factores } \\
\text { pre-exponenciales }\end{array}$} & \multicolumn{2}{|c|}{ Energias de activación } & \multicolumn{2}{|c|}{$\begin{array}{c}\text { Temperatura de referencia y } \\
\text { constantes de adsorción }\end{array}$} \\
\hline $\boldsymbol{k}_{1, \text { ref }}\left[\mathbf{s}^{-1}\right]$ & $6.6210^{1}$ & $E_{1}[\mathbf{J} / \mathbf{m o l}]$ & $1.4810^{5}$ & $T_{\text {ref,cin }}[\mathbf{K}]$ & 448 \\
\hline $\boldsymbol{k}_{2, \text { ref }}\left[\mathbf{s}^{-1}\right]$ & $1.8110^{3}$ & $E_{2}[\mathrm{~J} / \mathrm{mol}]$ & $1.1010^{5}$ & $K_{1}\left[\mathrm{~m}^{3} / \mathrm{mol}\right]$ & $4.1910^{1}$ \\
\hline $\boldsymbol{k}_{3, \text { ref }}\left[\mathbf{s}^{-1}\right]$ & $1.2210^{1}$ & $E_{3}[\mathbf{J} / \mathbf{m o l}]$ & $1.6910^{5}$ & $K_{3}\left[\mathrm{~m}^{3} / \mathrm{mol}\right]$ & $6.7510^{1}$ \\
\hline
\end{tabular}

Los parámetros cinéticos involucrados corresponden a los informados en la Tabla 3.3 (Campesi y col., 2012b).

Tabla 3.3: parámetros cinéticos. 


\subsection{Modelo para el sistema de inversión de flujo}

Para la simulación del sistema de inversión de flujo (ambos esquemas) se consideró un modelo unidimensional heterogéneo, sin incluir en forma explícita un término de dispersión axial. Se despreció la acumulación de materia de los reactivos en la fase sólida, la acumulación global de materia en la fase gaseosa, la eventual reacción homogénea y las pérdidas de energía hacia el exterior. Adicionalmente, se consideró que el material inerte no es adsorbente.

Debido al bajo contenido de VOCs presentes en la corriente a tratar (menor al 1\%), la misma se consideró como aire puro para el cálculo de sus propiedades fisicas, asumiendo además comportamiento de gas ideal y despreciando la variación del caudal molar total por las reacciones químicas.

Por otra parte, a los fines de simplificar la resolución de los balances de materia y energía, la presión se consideró constante e igual al valor de alimentación para los mismos. La pérdida de carga se evalúo considerando el valor medio temporal y espacial de la temperatura del gas en cada región. Adicionalmente, se consideró que el caudal se distribuye uniformemente en la sección transversal.

Finalmente, la temperatura del sólido se consideró uniforme en la sección transversal de cada canal y que cada uno de ellos es representativo del resto.

Para la zona donde hay material catalítico resulta:

Balance de energía para la fase sólida (región catalitica):

$$
\left(1-\varepsilon_{L, C}\right)\left(\rho c_{p}\right)_{S, C} \frac{\partial T_{S}}{\partial t}=a_{v, C} h_{m o d, C}\left(T_{G}-T_{S}\right)+a_{v, C} \ell \sum_{j=1}^{3}\left(-\Delta H_{j}^{\#}\right) r_{j}^{e f}
$$

donde $t$ es el tiempo, $T_{S}$ y $T_{G}$ la temperatura del sólido y del gas respectivamente, $a_{v, C}$ el área total de transferencia por unidad de volumen total, $h_{\text {mod,C }}$ el coeficiente de transferencia de energia modificado (ver Ec. 3.10), $\ell$ la longitud característica del catalizador (relación volumen de depósito/área de transferencia de masa), $\Delta H_{j}^{\#}$ y $r_{j}^{e f}$ la entalpía de combustión y la velocidad de consumo efectiva del componente " $j$ ", respectivamente. El subíndice " $C$ " en los 
diferentes parámetros se incluye para indicar que corresponden a la región catalítica, contemplando la posibilidad de diferentes configuraciones/materiales entre la misma y la región inerte.

Cabe aclarar que en la Ec. (3.9) se ha considerado $\sum_{i=1}^{3}\left(-\Delta H_{i}\right) r_{i}^{e f}=$ $\sum_{j=1}^{3}\left(-\Delta H_{j}^{\#}\right) r_{j}^{e f}$, siendo $\Delta H_{i}$ y $r_{i}^{e f}$ la entalpía y velocidad de reacción efectiva de la reacción “i”, lo cual se desprende fácilmente del análisis del sistema reactivo.

El coeficiente de transferencia de energía modificado, corresponde a una adaptación de lo propuesto por Vortmeyer y Schaefer (1974), que permite incorporar el efecto de la conducción térmica axial del sólido, de acuerdo con:

$$
\frac{1}{h_{\bmod }}=\frac{1}{h}+\frac{a_{v} \lambda_{S}}{\left(G c_{p G}\right)^{2}}
$$

siendo $c_{p G}$ el calor específico del gas, $\lambda_{S}$ la conductividad térmica axial del sólido (tomada en todos los casos como la correspondiente a la cordierita), $G$ la velocidad másica referida a la sección transversal total de la región considerada y $h$ el coeficiente de transferencia de energía en el canal (ver Apéndice 3.1).

Balance de energía en la fase gaseosa (región catalitica):

$$
\varepsilon_{L, C}\left(\rho c_{p}\right)_{G} \frac{\partial T_{G}}{\partial t}=-G c_{p G} \frac{\partial T_{G}}{\partial z}-a_{v, C} h_{m o d, C}\left(T_{G}-T_{S}\right)
$$

donde $\left(\rho c_{p}\right)_{G}$ es la capacidad calorífica por unidad de volumen de gas y $z$ la posición axial en el canal.

Balance de materia por componente en la fase gaseosa (región catalitica):

$$
\varepsilon_{L, C} \frac{\partial\left(C_{T} y_{j, G}\right)}{\partial t}=-\frac{G}{M_{\text {aire }}} \frac{\partial y_{j, G}}{\partial z}-a_{v, C} k_{m, j, C} C_{T}\left(y_{j, G}-y_{j, S}\right) \quad j=1 . .3
$$


donde $C_{T}$ es la concentración molar total en la fase gaseosa, $y_{j, G}$ e $y_{j, S}$ la fracción molar del componente “j” en la fase gaseosa y en la superficie del sólido respectivamente, $M_{\text {aire }}$ el peso molecular del aire y $k_{m, j, C}$ el coeficiente de transferencia de materia para el componente “ $j$ ”.

Balance de materia por componente en la fase sólida (región catalítica):

$$
k_{m, j, C} C_{T}\left(y_{j, G}-y_{j, S}\right)-\ell r_{j}^{e f}=0 \quad j=1 . .3
$$

Por otro lado, en la región donde solo existe material inerte se pueden utilizar los mismos balances dados por las Ecs. (3.9) a (3.12) con las siguientes modificaciones:

En la Ec. (3.9), se debe eliminar la liberación de energía por reacción química, con lo cual se obtiene:

Balance de energía para la fase sólida (región inerte):

$$
\left(1-\varepsilon_{L, I}\right)\left(\rho c_{p}\right)_{S, I} \frac{\partial T_{S}}{\partial t}=a_{\nu, I} h_{m o d, I}\left(T_{G}-T_{S}\right)
$$

donde el subíndice " $I$ " en los diferentes parámetros indica que corresponden a la región inerte.

La Ec. (3.11) no se ve alterada, a menos de las propiedades si fuera necesario, es decir:

Balance de energía en la fase gaseosa (región inerte):

$$
\varepsilon_{L, I}\left(\rho c_{p}\right)_{G} \frac{\partial T_{G}}{\partial t}=-G c_{p G} \frac{\partial T_{G}}{\partial z}-a_{\nu, I} h_{\mathrm{mod}, I}\left(T_{G}-T_{S}\right)
$$

Finalmente, en la Ec. (3.12), se debe eliminar el término de transferencia de materia, pues se supone que el sólido no es adsorbente, con lo cual se obtiene: 
Balance de materia por componente en la fase gaseosa (región inerte):

$$
\varepsilon_{L, I} \frac{\partial\left(C_{T} y_{j, G}\right)}{\partial t}=-\frac{G}{M_{\text {aire }}} \frac{\partial y_{j, G}}{\partial z} \quad j=1 . .3
$$

Conociendo las condiciones iniciales y de contorno (ver Secciones 3.3.1 y 3.3.2), lo desarrollado hasta aquí permitiría conocer la evolución temporal del sistema de inversión de flujo convencional por completo, es decir, se tendría el recorrido del sistema desde el arranque hasta el estado estacionario cíclico (css). Sin embargo, esto no será válido para el sistema de inversión de flujo rotativo, debido a que ineludiblemente la evolución temporal de cada canal, previo a alcanzar el css, dependerá de la posición angular en que se encontraba en el arranque del equipo. Para entender esto, puede ser útil la representación dada en la Figura 3.3, en donde se muestran dos grupos de canales que a tiempo cero se encuentran en dos posiciones angulares diferentes, $\mathrm{p}_{1} \mathrm{y} \mathrm{p}_{2}$.

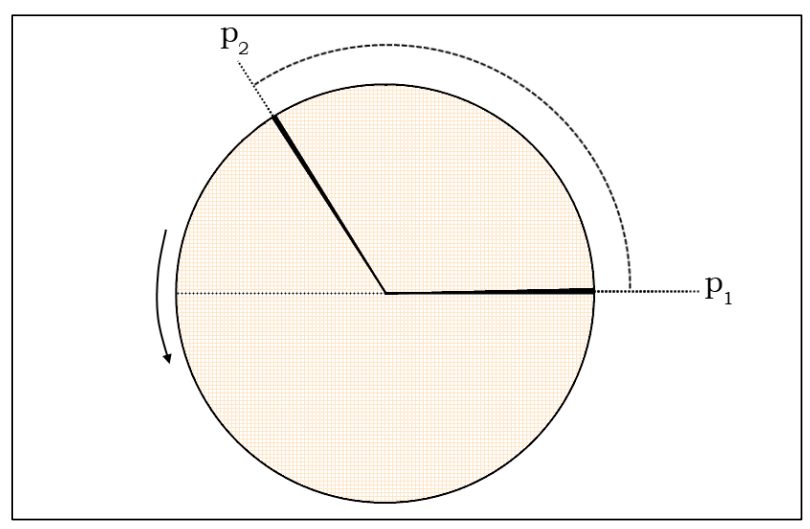

Figura 3.3: representación esquemática de la posición angular de una serie de canales (se ha omitido la región de limpieza para mayor claridad).

Suponiendo que el sistema se arrancara con una temperatura en el sólido constante y uniforme en todo el lecho (caso habitual), si se sigue la evolución temporal de las variables en la serie de canales sobre la posición angular $\mathrm{p}_{1}$ se podrá notar que su comportamiento será diferente de las variables en los canales que se encontraban en la posición $\mathrm{p}_{2}$. Esto se debe a que en los canales sobre $\mathrm{p}_{2}$, el sentido de circulación se invirtió antes (pero la condición inicial 
para ambos fue idéntica). De hecho, la evolución temporal de los canales que arrancaron en $\mathrm{p}_{2}$ será igual a la evolución de los canales en $\mathrm{p}_{1}$ solo en el intervalo de tiempo para el cual el sistema rotó un ángulo $\pi-\mathrm{p}_{2}$ (y luego en el css).

De todas maneras, se puede razonar que una vez alcanzado el css, lo que le ocurre a las variables en un canal ubicado en una posición angular cualquiera, sí será representativo de lo que le ocurre al resto (con el desfasaje temporal que le corresponda según la diferencia en la posición angular). Teniendo lo anterior en mente y considerando que en este estudio no se pretende conocer la evolución dinámica real del sistema sino solo el css, se puede establecer un mecanismo para evitar lo primero, simulando solo la evolución temporal de las variables de un canal que inicialmente se encuentra en la posición $\mathrm{p}_{1}$ de la Figura 3.3. De esta manera, el estado cíclicamente estacionario determinado para ese canal será equivalente al de cualquier otro, independientemente del tiempo (real) que pueda tardar en alcanzarlo un canal que se hubiera encontrado en otra posición angular durante el arranque del equipo.

Teniendo en cuenta lo discutido en los párrafos anteriores, se puede concluir que si la conducción en sentido angular y radial en el sistema de inversión de flujo rotativo resulta despreciable, lo desarrollado hasta aquí será un modelo general para la determinación del css en ambos esquemas de inversión. Cabe resaltar no obstante que, en los balances dados por las expresiones (3.9) a (3.16), se deberá tener en cuenta que el flujo másico será diferente dependiendo de la etapa considerada en el esquema rotativo (de reacción o de limpieza). Esta situación no se discriminó en las expresiones de los balances en pos de una mayor claridad de los mismos. La determinación del caudal necesario para la etapa de limpieza se presenta en el Capítulo 4.

Para completar el modelo restan definir las condiciones iniciales, de contorno y la relación que establece la inversión del sentido de circulación. Esta última puede considerarse asociando un signo a las velocidades másicas en las Ecs. (3.11), (3.12), (3.15) y (3.16), que dependerá del semiciclo a simular. Otra posibilidad es espejar los perfiles obtenidos al final del semiciclo anterior sobre la variable axial, tomando el resultado como la condición inicial del semiciclo a simular. Esta última opción es la alternativa utilizada con mayor frecuencia (Bunimovich y Sapoundjiev, 2013) y ha sido adoptada aquí. 


\subsubsection{Condiciones iniciales y de contorno para el sistema de inversión de flujo convencional}

Las condiciones de contorno resultan independientes del semiciclo considerado y del tiempo, quedando determinadas por la temperatura y la fracción molar de cada componente en la corriente a tratar, $T_{G}^{0}$ e $y_{j, G}^{0}$, respectivamente. Se debe recordar que dichos valores no se modifican entre semiciclos (las condiciones de alimentación al sistema se consideran constantes en todos los casos), pero si se modifica su "posición" (es decir, la alimentación proviene de uno u otro lado, dependiendo de qué semiciclo se considere).

Si el lecho contiene inerte en sus extremos, se debe establecer una condición adicional que permita vincular las variables en los límites de dichas regiones y la región catalítica. Esta vinculación viene dada por la continuidad en las variables de la corriente gaseosa (temperatura y fracciones molares). La condición análoga para las variables de la fase sólida no se verifica: las fracciones molares en dicha fase presentarán un quiebre abrupto desde cero (el inerte se considera no adsorbente) al valor que dicte la velocidad de reacción; la temperatura también presentará un quiebre como consecuencia de la energía liberada por la reacción química y la ausencia de términos explícitos de conducción en el sólido.

Por otro lado, recordando que las condiciones iniciales dependerán del semiciclo considerado como consecuencia de la inversión del sentido de circulación (a menos del primer semiciclo a simular), se tiene:

$$
\begin{aligned}
& T_{S}^{n s c}(0, z)=\left\{\begin{array}{lll}
T_{S}^{i n i} & \text { si } & n s c=1 \\
T_{S}^{n s c-1}\left(t_{\text {ciclo }} / 2, L_{e q}-z\right) & \text { si } & n s c>1
\end{array}\right. \\
& T_{G}^{n s c}(0, z)=\left\{\begin{array}{lll}
T_{G}^{i n i} & \text { si } & n s c=1 \\
T_{G}^{n s c-1}\left(t_{\text {ciclo }} / 2, L_{e q}-z\right) & \text { si } & n s c>1
\end{array}\right. \\
& y_{j, G}^{n s c}(0, z)=\left\{\begin{array}{lll}
y_{j, G}^{i n i} & \text { si } & n s c=1 \\
y_{j, G}^{n s c-1}\left(t_{\text {ciclo }} / 2, L_{e q}-z\right) & \text { si } & n s c>1
\end{array}\right.
\end{aligned}
$$


donde $y_{j, G}^{i n i}, T_{S}^{i n i}$ y $T_{G}^{i n i}$ corresponden a los valores iniciales (para el primer semiciclo y en todo el lecho) de las fracciones molares de cada componente en el gas y de la temperatura del sólido y del gas, respectivamente; $L_{e q}$ representa la longitud total del equipo y el superíndice " $n s c$ " indica el semiciclo actual a simular. Los valores adoptados para $T_{S}^{i n i}, T_{G}^{i n i}$ e $y_{j, G}^{i n i}$ se discutirán en el Capítulo 4 .

\subsubsection{Condiciones iniciales y de contorno para el sistema de inversión de flujo rotativo}

La existencia de la etapa de limpieza requiere de la definición de un grupo adicional de condiciones iniciales y de contorno. Así, la temperatura y la fracción molar de cada componente en la corriente de gas a tratar, determinan las condiciones de contorno (alimentación) de la etapa de reacción, debiendo plantear también una condición de continuidad en las variables de la fase gaseosa si hay material inerte en los extremos.

Adicionalmente, recordando el esquema mostrado en la Figura 3.1, se puede ver que la temperatura y la fracción molar de cada componente en la salida de la etapa de reacción, $\left\langle T_{G}\right\rangle^{E R}$ e $\left\langle y_{j, G}\right\rangle^{E R}$ respectivamente, determinan las condiciones de contorno (alimentación) para la etapa de limpieza. Planteando sendos balances de energía y materia para el caudal que egresa de cada canal (recordar que en el css la respuesta temporal quedará asociada a la posición angular), se tiene:

$$
t_{E R} \int_{T_{\text {ref }}}^{\left\langle\left. T_{G}\right|^{E R}\right.} c_{p G}(T) d T=\int_{0}^{t_{E R}}\left[\int_{T_{\text {ref }}}^{T_{G}\left(t, L_{\text {eq }}\right)} c_{p G}(T) d T\right] d t
$$

o, en términos del calor específico medio, $\bar{c}_{p G}$ (ver Apéndice 3.1): 


$$
\begin{gathered}
\bar{c}_{p G}\left(\left\langle T_{G}\right\rangle^{E R}, T_{r e f}\right)\left(\left\langle T_{G}\right\rangle^{E R}-T_{r e f}\right)= \\
\frac{1}{t_{E R}} \int_{0}^{t_{E R}} \bar{c}_{p G}\left(T_{G}\left(t, L_{e q}\right), T_{r e f}\right)\left(T_{G}\left(t, L_{e q}\right)-T_{r e f}\right) d t \\
\left\langle y_{j, G}\right\rangle^{E R}=\frac{1}{t_{E R}} \int_{0}^{t_{E R}} y_{j, G}\left(t, L_{e q}\right) d t
\end{gathered}
$$

donde $T_{\text {ref }}$ es una temperatura de referencia.

Las condiciones iniciales en esta etapa corresponden directamente a las condiciones finales en la etapa de reacción, sin invertir el sentido. Por otro lado, recordando que se toma como referencia la serie de canales que a tiempo cero están justo en la posición donde se invierte el flujo (posición $\mathrm{p}_{1}$ de la Figura 3.3), la condición de inversión viene dada por expresiones completamente análogas a las (CI3.1) a (CI3.3), pero considerando que se debe invertir los perfiles finales de la etapa de limpieza y no de reacción (es decir, se debe reemplazar $t_{\text {ciclo }} / 2$ por $\left.t_{E R}+t_{E L}\right)$.

\subsubsection{Pérdida de carga}

Para el cálculo de la pérdida de carga en cada región se consideró que el régimen de flujo en cada canal corresponde al régimen laminar, para lo cual deberá verificarse $\operatorname{Re} \lesssim 2000$. Luego, evaluando las propiedades de la corriente a la temperatura media temporal y espacial de cada región y despreciando los términos asociados a las variaciones temporales:

$$
|\Delta P|=\frac{1}{8}(f \operatorname{Re}) \frac{L \mu_{G} G a_{v}^{2}}{\varepsilon_{L}^{3} \rho_{G}}
$$

donde $|\Delta P|$ es la caída de presión en la región considerada, $L$ la longitud de la misma, $(f \mathrm{Re})$ el producto entre el factor de Fanning y el número de Reynolds (constante dependiente de la geometría para régimen laminar) y $\mu_{G}$ la viscosi- 
dad de la corriente gaseosa. $\varepsilon_{L}, a_{v}, \rho_{G}$ y $G$, poseen el significado ya dado (solo que no se discriminó aquí por región).

\subsubsection{Contenido de VOCs a la salida del sistema}

Para el sistema de inversión de flujo convencional, el contenido de VOCs en la salida del sistema varía con el tiempo y corresponde directamente a las fracciones molares de cada componente evaluadas en la posición $z=L_{e q}$. Sin embargo, la información requerida habitualmente corresponde al promedio de las mismas sobre todo el semiciclo. Luego:

$$
\begin{aligned}
& \left\langle y_{j, G}\right\rangle=\frac{2}{t_{\text {ciclo }}} \int_{0}^{t_{\text {ciclo }} / 2} y_{j, G}\left(t, L_{e q}\right) d t \\
& \left\langle y_{\text {VOCs }}\right\rangle=\sum_{j=1}^{3}\left\langle y_{j, G}\right\rangle
\end{aligned}
$$

donde $\left\langle y_{j, G}\right\rangle$ es la fracción molar de cada componente y $\left\langle y_{V O C s}\right\rangle$ la fracción molar total de VOCs, ambas a la salida del sistema.

Por otro lado, para el sistema rotativo, el contenido de VOCs a la salida del mismo resulta invariante una vez alcanzado el css. De acuerdo con el esquema presentado en la Figura 3.1, el contenido de VOCs a la salida se obtiene a partir de un balance en el punto de mezcla. Recordando que el peso molecular de la corriente es (aproximadamente) invariable:

$$
\begin{aligned}
& \left\langle y_{j, G}\right\rangle=\left(1-G_{m, L} / G_{m, t o t}\right)\left\langle y_{j, G}\right\rangle^{E R}+G_{m, L} / G_{m, t o t}\left\langle y_{j, G}\right\rangle^{E L} \\
& \left\langle y_{j, G}\right\rangle^{E L}=\frac{1}{t_{E L}} \int_{t_{E R}}^{t_{E R}+t_{E L}} y_{j, G}\left(t, L_{e q}\right) d t \\
& \left\langle y_{\text {VOCs }}\right\rangle=\sum_{j=1}^{3}\left\langle y_{j, G}\right\rangle
\end{aligned}
$$


donde $\left\langle y_{j, G}\right\rangle^{E R},\left\langle y_{j, G}\right\rangle$ e $\left\langle y_{V O C s}\right\rangle$ poseen el mismo significado ya dado, $G_{m, t o t}$ es el caudal másico total de la corriente a tratar, $G_{m, L}$ es el caudal másico total derivado para la limpieza e $\left\langle y_{j, G}\right\rangle^{E L}$ es la fracción molar de cada componente a la salida de la región de limpieza. Para el cálculo de $\left\langle y_{j, G}\right\rangle^{E R}$, ver Ec. (3.18).

\subsubsection{Discusión del modelo}

Como fuera mencionado, el modelo utilizado para la simulación de los sistemas de inversión de flujo corresponde a un modelo unidimensional heterogéneo. En el mismo, se desprecian los efectos dispersivos, a menos de la conducción térmica de los sólidos en sentido axial, que es tenida en cuenta en forma aproximada a través de la expresión (3.10). Adicionalmente, se desprecian todas las contribuciones radiales (y angulares) y las pérdidas de energía hacia el exterior (sistema adiabático), así como la eventual reacción en fase gaseosa.

A fin de evaluar la validez del modelo escogido, se discutirán brevemente los dos modelos mayormente utilizados en bibliografia, el seudo-homogéneo y el heterogéneo, ambos unidimensionales. Como fue señalado por Matros y Bunimovich (1996), en los sistemas donde se forma un frente de onda que se mueve a lo largo del reactor, resulta imperativo tener en cuenta alguno de los dos mecanismos principales de disipación de energía en el reactor: la dispersión axial térmica o la velocidad finita de transferencia entre la fase sólida y la gaseosa.

En su forma más elemental, el modelo unidimensional seudo-homogéneo (velocidad infinita de transferencia entre el sólido y el gas) contempla un balance de energía global que incluye los siguientes términos: acumulación de energía, conducción axial térmica, transporte convectivo de energía y liberación de energía por reacción química. En cuanto al balance de materia por componente, este incluye solo dos términos: el transporte convectivo de materia y el consumo/generación por reacción química. Todos estos términos constituyen los elementos básicos para la simulación de un sistema de inver- 
sión de flujo y, por lo tanto, este modelo permite predecir el comportamiento del sistema, al menos cualitativamente.

El modelo anterior ha sido ampliamente estudiado. Un listado con las principales contribuciones al análisis del mismo aplicado a sistemas de inversión de flujo se puede hallar en Bunimovich y Sapoundjiev (2013). La mayor limitación es la propia de un modelo seudo-homogéneo: si las diferencias locales de temperatura y/o concentración entre la fase sólida y la gaseosa son significativas, el modelo no permitirá una adecuada predicción del estado del sistema; en particular, la velocidad de reacción no será correctamente evaluada. Estas diferencias serán tanto más elevadas cuanto más rápidas sean las reacciones involucradas o mayor sea el salto térmico adiabático de la corriente. Una forma aproximada de resolver tales deficiencias es utilizar la relación encontrada por Vortmeyer y Schaefer (1974), de donde se ha derivado la expresión (3.10). Tal relación permite tener en cuenta los dos mecanismos de disipación de energía, corrigiendo originalmente el coeficiente de dispersión axial térmica (conductividad térmica para los monolitos), contabilizando el efecto de la resistencia a la transferencia de energía entre las fases sólida y gaseosa. Expresiones análogas fueron utilizadas por van de Beld y Westerterp (1994), quienes compararon el desempeño del modelo seudo-homogéneo frente al heterogéneo (pero considerando un coeficiente de dispersión axial térmica en la fase gaseosa en lugar de la sólida). Los autores demostraron que dicha expresión resulta cualitativamente muy satisfactoria para bajas velocidades (bajos números de Reynolds), donde el término dominante es la dispersión axial (real). Por el contrario, cuando el término dominante es la resistencia a la transferencia de energía entre el sólido y el gas, esta aproximación no arrojará resultados precisos. En su lugar, es esperable que funcione mejor la forma dada en la expresión (3.10) asociada a un modelo heterogéneo, que corrige en este caso el término dominante. De hecho, Ruthven (1984) utilizó una formulación equivalente a la (3.10) para transferencia de materia en un proceso de adsorción, corrigiendo el coeficiente de transferencia de materia por el efecto de la dispersión axial de materia y verificó que esta corrección resulta muy adecuada para la situación descrita.

Cuando el modelo unidimensional seudo-homogéneo no resulta adecuado para la simulación del reactor, se debe seleccionar otro modelo con mayor 
grado de detalle, siendo la alternativa más frecuente el unidimensional heterogéneo. Este último ha sido sin duda el modelo más utilizado.

De acuerdo con Matros y col. (1999), un modelo como el desarrollado al inicio de la Sección 3.3, pero sin los términos de acumulación en la fase gaseosa (Ecs. 3.11 y 3.12) y sin la corrección dada por la expresión (3.10), incorpora todos los factores esenciales que afectan la dinámica de la operación de un reactor de inversión de flujo. Sin embargo, otros autores (e.g., Marín y col., 2010; Fissore y col., 2005) incluyen también términos de dispersión y acumulación en los balances de materia en ambas fases y en los balances de energía en la fase gaseosa. Adicionalmente, Fissore y col. (2005) incorpora un balance de materia global, siendo esto último muy poco usual en la simulación de los sistemas de inversión de flujo.

En particular, considerando que para el sistema bajo estudio la velocidad de las reacciones involucradas resulta muy elevada una vez superados los $\approx 200{ }^{\circ} \mathrm{C}$, es esperable que los gradientes de temperatura y concentración entre la fase sólida y la gaseosa no puedan despreciarse. Por esta razón, el modelo seudo-homogéneo fue descartado.

En cuanto a los efectos dispersivos en la fase gaseosa, los mismos se despreciaron debido a que solo serán relevantes si la velocidad del gas en el canal es muy baja (Matros y col., 1999), situación que presumiblemente no se verifique para dimensiones típicas de sistemas industriales. En forma similar, la influencia de la conductividad térmica en la fase sólida también queda vinculada a la velocidad del gas, por lo que es esperable que su impacto no sea significativo a altas velocidades y la expresión (3.10) sea adecuada, como ya fue mencionado.

Por otra parte, comparando los términos de acumulación en las expresiones (3.9) a (3.16), se puede ver que la inercia térmica del sólido es dominante en el comportamiento dinámico del sistema, debido a la diferencia entre la densidad del sólido y del gas (aproximadamente 3 órdenes de magnitud), mientras que la acumulación de materia en ambas fases y de energía en la fase gaseosa solo tendrá influencia al inicio de cada semiciclo y durante un tiempo muy breve en comparación a la escala de tiempo del sólido. Por esta razón, es usual que dichos términos se desprecien. No obstante, en el modelo desarrollado aquí se 
han retenido dichos términos para la fase gaseosa, ya que no se obtiene prácticamente ningún beneficio por su omisión.

Otro punto a analizar es la inclusión o no de la reacción en fase homogénea. Si bien algunos autores incorporan los términos de reacción homogénea en los balances de energía y materia para la fase gaseosa (e.g., Marin y col., 2005), la menor temperatura requerida por la reacción catalítica permite despreciar dicho término en cualquier condición de operación realista.

En cuanto a desestimar las pérdidas de energía hacia el exterior, las mismas están directamente relacionadas a la suposición de adiabaticidad. La omisión de estos efectos está fundamentada en las dimensiones esperables de los equipos, por tratarse de sistemas industriales. No obstante, si se necesitase cuantificar el posible efecto de las pérdidas de energía, se podría incorporar un término de transferencia hacia el exterior. En general, los trabajos en donde normalmente se incluyen efectos de este tipo están asociados al estudio de sistemas a escala de laboratorio, donde los menores diámetros del equipo dificultan notablemente la posibilidad de aislar térmicamente el mismo (e.g., Hevia y col., 2007; Cittadini y col., 2001). En estos casos, es usual incorporar también un balance de energía en la pared del sólido que delimita el reactor, debido a que el mismo afecta la dinámica del sistema.

Cabe aclarar que la suposición de adiabaticidad no puede desligarse del contenido de VOCs. Es decir, si bien en términos absolutos la condición de adiabaticidad solo dependerá del nivel térmico del sistema (para dimensiones definidas de los equipos), la forma en que las pérdidas de energía impactan sobre la operación del mismo sí dependerá del contenido de VOCs, pues define la energía liberada por unidad de volumen. Teniendo en cuenta lo anterior, nos referiremos a la condición de adiabaticidad en términos relativos al impacto que las pérdidas de energía pudieran tener sobre la operación del sistema y no en términos absolutos. De esta manera, se puede afirmar que en la práctica existirá un límite en el mínimo contenido de VOCs para que un modelo adiabático pueda ser efectivamente adecuado para simular la operación, aún para dimensiones típicas de sistemas industriales. De acuerdo con Matros y col. (1996), dicho limite corresponde normalmente a un $\Delta T_{a d} \approx 15^{\circ} \mathrm{C}$.

Cuando el contenido de VOCs es cercano o menor a dicho valor, la operación no podrá ser perfectamente adiabática, llegando inclusive a no ser posible 
la presencia de un estado encendido. Adicionalmente, en aquellos casos en que las pérdidas de energia al exterior son relevantes, se formarán perfiles radiales de temperatura, pudiendo ser necesaria su inclusión en el modelo. Lógicamente, la información obtenida en tal caso será más detallada y permitirá estimar con mayor precisión el límite mencionado anteriormente en el contenido de VOCs. Al respecto, Bunimovich y col. (1990) utilizaron un modelo bidimensional heterogéneo, para estudiar el efecto que pudiera tener la presencia de perfiles radiales de temperatura en un reactor de inversión de flujo de un metro de diámetro, cuando se procesan sistemas reaccionantes de muy baja liberación de energía por reacción química. Los autores encontraron que aun siendo pequeña, la pérdida de energia hacia el exterior puede llevar a la extinción de la reacción como consecuencia de la formación, y posterior migración, de una zona fría en las vecindades de la pared del reactor. Según informan los autores, en esa condición limite, dicho proceso puede inclusive tomar varios dias.

Por otro lado, Salomons (2003) estudia el tratamiento de corrientes de aire contaminadas con metano en un equipo de inversión de flujo de $20 \mathrm{~cm}$ de diámetro interno, tanto en forma experimental como a través de la simulación matemática. Para esto último utiliza dos modelos, uno unidimensional heterogéneo y otro bidimensional heterogéneo y compara la capacidad de los mismos para ajustar los valores obtenidos experimentalmente. El autor concluye que el ajuste del modelo unidimensional es pobre frente al bidimensional. La conclusión, no obstante, no es válida porque se basa en una inconsistencia, debido a que en el modelo unidimensional considerado por el autor se desprecian las pérdidas de energía al exterior (reactor adiabático), mientras que en el bidimensional son tenidas en cuenta.

En base a lo discutido, se puede concluir que el modelo propuesto será adecuado para las condiciones a analizar en el presente estudio. De todas maneras, si en el diseño de los equipos el diámetro de alguno de ellos resultara pequeño ( $\lesssim 1 \mathrm{~m}$ ), debería estimarse la pérdida de energía al exterior a fin de verificar la suposición de adiabaticidad. 


\subsubsection{Análisis particular del sistema rotativo}

Lo discutido previamente resulta general para ambos sistemas de inversión de flujo. Sin embargo, para el sistema rotativo se requiere una discusión adicional.

Para que el modelo utilizado sea válido, es necesario suponer que no hay transferencia de energía de un canal a sus vecinos más cercanos y que, consecuentemente, la evolución de las variables en un canal es representativa de todos (es decir, cada canal es independiente). Bajo esta suposición, si se observa la sección transversal del equipo en un instante dado, se verá que existe una amplia variación espacial de la temperatura en los canales en la dirección angular, como consecuencia de la rotación del equipo para emular la inversión del flujo. Una vez alcanzado el css, dicha variación espacial de la temperatura será independiente del tiempo considerado. Teniendo presente esto, se puede razonar que, si el material que constituye la matriz monolitica presenta una elevada conductividad térmica, habrá componentes de conducción en sentido angulares. De ser estos importantes, podrian invalidar la suposición realizada sobre la independencia de los canales, ya que al ser la longitud de transferencia dependiente de la posición radial, la temperatura tendería a ser más uniforme hacia el centro del equipo, generándose también un gradiente radial, con la consecuente transferencia en dicha dirección.

En principio, si este problema fuera importante, se debería utilizar un modelo que permitiera tener en cuenta la variación de la temperatura en toda la matriz sólida, incluyendo componentes de conducción térmica en sentido angular y radial, aun cuando en cada canal se utilizara un modelo unidimensional respecto del comportamiento del gas. Cabe aclarar que en la bibliografia consultada, no se ha detectado que el esquema de inversión de flujo rotativo haya sido explorado, habiéndose encontrado solo dos menciones breves al mismo (Kolios y col., 2000; Eigenberger y Nieken, 1994), pero en ninguna de estas referencias se propone o discute un modelo. No obstante, se puede razonar que si la conducción angular fuese significativa, la uniformización de la temperatura en sentido transversal sería beneficiosa para este sistema, ya que al ingresar al equipo la misma corriente en sentidos opuestos (ver Figura 3.1) y siendo el comportamiento de cada una de ellas equivalente entre sí, el sistema tendería a comportarse como el reactor en contracorriente 
descrito por Eigenberger y Nieken (1994), el cual será abordado en la Sección 3.5. Por lo tanto, si la conducción angular fuera importante, el error que se cometería al no considerar dicho efecto correspondería a una situación "conservativa". De todas maneras, considerando que en este trabajo se plantea el uso de un material cerámico, significativamente menos conductivo que los metales, es esperable que la conducción angular, si bien presente, sea despreciable (y por lo tanto, también lo sea la radial).

Otra suposición que puede ser más objetable en este sistema que en el convencional es la distribución uniforme del flujo. En el sistema convencional los problemas de distribución de flujo estarán asociados estrictamente a un problema de tipo práctico (por ejemplo, defectos en el diseño de los cabezales). En contraposición, si para el sistema rotativo se visualiza nuevamente la sección transversal del equipo en cualquier instante, la variación espacial de la temperatura traerá aparejado una variación de la densidad y viscosidad del gas en la sección transversal. Considerando que la caída de presión en cada canal depende de estos parámetros (ver Ec. 3.19) y que debe ser independiente del canal observado, puede pensarse que esto generaría una distribución no uniforme del flujo, ya no como consecuencia de un defecto en el diseño del equipo, sino como algo inherente al sistema. No obstante, recordando nuevamente el esquema presentado en la Figura 3.1, cualquier canal que se ubique en una posición angular dada, poseerá un perfil de temperatura completamente espejado respecto del perfil presente en un canal ubicado exactamente a $180^{\circ}$ de distancia (al menos en el css), con lo cual se puede reconocer una cierta simetría en el sistema. Esto permite prever que la densidad y la viscosidad promediadas a lo largo de la posición axial en cada canal para un instante dado, no varien significativamente de un canal a otro (aunque si lo hagan en la sección transversal). En esas circunstancias, es esperable que el caudal que circule por cada canal sea aproximadamente igual en cada uno.

\subsection{Modelo para el sistema regenerador-reactor}

Previo a la descripción del modelo considerado para este sistema (Figura 3.2), es menester aclarar algunos aspectos particulares. 
Los materiales utilizados en los intercambiadores de calor rotativos comerciales son usualmente metales, principalmente aluminio si las temperaturas requeridas son bajas o acero cuando las temperaturas son mayores (Product Catalogue - Heat Exchanger, 2013; Regenerative Heat Exchangers For Industry and Process Technology, 2003). A pesar de esto, en el presente estudio se consideró para el material del intercambiador, el mismo material cerámico informado en la Sección 3.2 (cordierita). Esta elección se realizó con el fin de establecer un punto de comparación entre los sistemas de inversión de flujo y el sistema regenerador-reactor que sea equivalente. A pesar de que los materiales cerámicos no son comúnmente utilizados en los equipos comerciales, puede suponerse que esto no responde a una dificultad técnica sino a desventajas de tipo económica (mayor costo del material y mayor costo de construcción). De hecho, para regeneradores que requieren una elevada temperatura de operación (mayor a unos $800^{\circ} \mathrm{C}$ ) se han planteado alternativas considerando estructuras monolíticas de cerámica, tipo alúmina (Willmott, 2002).

En lo concerniente a la simulación del sistema aparece, en principio, una doble problemática. Por un lado, en el regenerador se presenta una situación similar a la descrita para el sistema de inversión de flujo rotativo, respecto de la evolución temporal de las variables en un canal dado durante el arranque, dependiendo de la posición angular (ver Figura 3.3 y discusión asociada). Sin embargo, recordando nuevamente que el interés en este estudio está sobre el css y no sobre la evolución dinámica real del sistema hasta alcanzar dicho estado, se adoptó para este sistema un camino equivalente al tomado para el sistema de inversión de flujo rotativo, simulando solo lo que ocurre con un canal que en el comienzo de la operación estuviese en la posición angular indicada como $\mathrm{p}_{1}$ en la Figura 3.3 y teniendo en cuenta que dicho canal será representativo del resto en el css.

El otro inconveniente se da en la simulación del reactor. Si se visualiza nuevamente el arranque del sistema, se podrá concluir que las condiciones de alimentación al reactor variarán mientras dure el estado transitorio. Como consecuencia, la operación de este último también será no-estacionaria. No obstante, recordando nuevamente que en este estudio no se pretende conocer la evolución temporal del sistema previo a alcanzar el css, se omitió este aspecto. En su lugar, se consideró que el reactor opera en estado estacionario y 
que la temperatura de alimentación al mismo viene dada por la temperatura promedio a la salida del regenerador, calculada de acuerdo a la evolución indicada en el párrafo anterior. Para una discusión más detallada sobre estos aspectos, ver la Sección 3.4.5.

\subsubsection{Modelo para el regenerador}

Teniendo presente lo mencionado en los párrafos anteriores y considerando que el significado de cada variable es idéntico al mencionado en la Sección 3.3 para el inerte, pero aplicado al regenerador, los balances quedan representados mediante el siguiente conjunto de ecuaciones diferenciales parciales y algebraicas:

Balance de energía en la fase sólida (regenerador):

$$
\left(1-\varepsilon_{L, I}\right)\left(\rho c_{p}\right)_{S, I} \frac{\partial T_{S}}{\partial t}=a_{v, I} h_{m o d, I}\left(T_{G}-T_{S}\right)
$$

Balance de energía en la fase gaseosa (regenerador):

$$
\varepsilon_{L, I}\left(\rho c_{p}\right)_{G} \frac{\partial T_{G}}{\partial t}=-G_{I} c_{p G} \frac{\partial T_{G}}{\partial z}-a_{v, I} h_{m o d, I}\left(T_{G}-T_{S}\right)
$$

donde la velocidad másica $G_{I}$ dependerá de la etapa considerada (enfriamiento, limpieza o calentamiento), pero al igual que antes se optó por no hacer una discriminación explícita de ello para facilitar la lectura de cada balance. Nuevamente, la determinación del caudal utilizado para la limpieza se presenta en el Capítulo 4.

Balance de materia por componente en la fase gaseosa (regenerador):

$$
\varepsilon_{L, I} \frac{\partial\left(C_{T} y_{j, G}\right)}{\partial t}=-\frac{G_{I}}{M_{\text {aire }}} \frac{\partial y_{j, G}}{\partial z} \quad j=1 . .3
$$


En cuanto a las condiciones de contorno, en forma similar al sistema de inversión de flujo rotativo, las mismas dependerán de la región considerada. Para la región de enfriamiento quedan determinadas por la temperatura y la fracción molar de cada componente en la corriente a tratar. Para la región de limpieza, como se hace circular aire puro (ver Figura 3.2), la fracción molar de cada componente en la corriente gaseosa alimentada es nula, mientras que la temperatura se considera igual a la temperatura de la corriente de gas a tratar. Por último, para la región de calentamiento, las condiciones de contorno quedan determinadas por la temperatura y la fracción molar de cada componente en la corriente efluente del reactor.

Por otro lado, recordando que para la simulación se considera un canal ubicado en la posición indicada como $\mathrm{p}_{1}$ en la Figura 3.3, las condiciones iniciales quedarán definidas por:

Condiciones iniciales para la región de calentamiento:

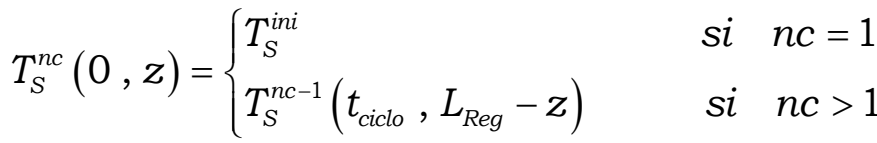

$$
\begin{aligned}
& T_{G}^{n c}(0, z)=\left\{\begin{array}{lrl}
T_{G}^{i n i} & \text { si } & n c=1 \\
T_{G}^{n c-1}\left(t_{\text {ciclo }}, L_{\text {Reg }}-z\right) & \text { si } & n c>1
\end{array}\right. \\
& y_{j, G}^{n c}(0, z)=\left\{\begin{array}{lrl}
y_{j, G}^{i n i} & \text { si } & n c=1 \\
y_{j, G}^{n c-1}\left(t_{c i c l o}, L_{\text {Reg }}-z\right) & \text { si } & n c>1
\end{array}\right.
\end{aligned}
$$

donde, al igual que para los sistemas de inversión de flujo, $T_{S}^{i n i}, T_{G}^{i n i}$ e $y_{j, G}^{i n i}$ corresponden a los valores iniciales (primer ciclo) de la temperatura del sólido y del gas y de las fracciones molares de cada componente en el gas, $L_{\text {Reg }}$ corresponde a la longitud total del regenerador y el superíndice $n c$ indica el ciclo actual a simular.

En forma similar al sistema de inversión de flujo rotativo, las condiciones iniciales para la etapa de limpieza corresponden directamente a las condiciones finales en la etapa de enfriamiento, sin invertir el sentido. Por otro lado, 
las condiciones iniciales en la etapa de calentamiento corresponden a las finales de la etapa de limpieza invertidas en sentido.

\subsubsection{Modelo para el reactor}

Recordando que en la simulación de este equipo no se consideró la variación de las condiciones de alimentación al mismo, los balances se plantean ignorando los términos de acumulación.

Luego, para el reactor se tiene un conjunto de ecuaciones diferenciales ordinarias y algebraicas, dado por:

Balance de energía en la fase sólida (reactor):

$$
h_{\text {mod,C }}\left(T_{G}-T_{S}\right)+\ell \sum_{j=1}^{3}\left(-\Delta H_{j}^{\#}\right) r_{j}^{e f}=0
$$

Balance de energía en la fase gaseosa (reactor):

$$
G_{C} c_{p G} \frac{d T_{G}}{d z}=-a_{v, C} h_{m o d, C}\left(T_{G}-T_{S}\right)
$$

Balance de materia por componente en la fase gaseosa (reactor):

$$
\frac{G_{C}}{M_{\text {aire }}} \frac{d y_{j, G}}{d z}=-a_{v, C} k_{m, j, C} C_{T}\left(y_{j, G}-y_{j, S}\right) \quad j=1 . .3
$$

Balance de materia por componente en la fase sólida (reactor):

$$
k_{m, j, C} C_{T}\left(y_{j, G}-y_{j, S}\right)-\ell r_{j}^{e f}=0 \quad j=1 . .3
$$

Las condiciones de contorno para el reactor quedan definidas por las características de la corriente de alimentación al mismo, la cual corresponde a la combinación de las corrientes de salida de las etapas de enfriamiento y limpieza del regenerador (ver Figura 3.2), resultando constantes en el css. Se 
deberán evaluar la temperatura y las fracciones molares en la alimentación al reactor, $T_{G}^{0, R}$ e $y_{j, G}^{0, R}$ respectivamente, para lo cual será necesario plantear un balance de energía y otro de materia en las corrientes de salida de las regiones de enfriamiento y limpieza (análogas a las Ec. 3.17 y 3.18) y luego plantear el balance de energía y materia en el punto de mezcla. Es decir:

$$
\begin{gathered}
\bar{c}_{p G}\left(\left\langle T_{G}\right\rangle^{E E}, T_{r e f}\right)\left(\left\langle T_{G}\right\rangle^{E E}-T_{r e f}\right)= \\
\frac{1}{t_{E E}} \int_{0}^{t_{E E}} \bar{c}_{p G}\left(T_{G}\left(t, L_{R e g}\right), T_{r e f}\right)\left(T_{G}\left(t, L_{R e g}\right)-T_{r e f}\right) d t \\
\bar{c}_{p G}\left(\left\langle T_{G}\right\rangle^{E L}, T_{r e f}\right)\left(\left\langle T_{G}\right\rangle^{E L}-T_{r e f}\right)= \\
\frac{1}{t_{E L}} \int_{t_{E E}}^{t_{E E}+t_{E L}} \bar{c}_{p G}\left(T_{G}\left(t, L_{R e g}\right), T_{r e f}\right)\left(T_{G}\left(t, L_{R e g}\right)-T_{r e f}\right) d t \\
G_{m, t o t} \bar{c}_{p G}\left(T_{G}^{0, R},\left\langle T_{G}\right\rangle^{E E}\right)\left(T_{G}^{0, R}-\left\langle T_{G}\right\rangle^{E E}\right)= \\
G_{m, L} \bar{c}_{p G}\left(\left\langle T_{G}\right\rangle^{E L}, T_{G}^{0, R}\right)\left(\left\langle T_{G}\right\rangle^{E L}-T_{G}^{0, R}\right)
\end{gathered}
$$

siendo $\left\langle T_{G}\right\rangle^{E E}$ y $\left\langle T_{G}\right\rangle^{E L}$, el promedio temporal de la temperatura del gas en la salida de la región de enfriamiento del sólido y limpieza, respectivamente, $G_{m, t o t}$ el caudal másico total de la corriente a tratar y $G_{m, L}$ el caudal másico total utilizado para la limpieza.

$$
\begin{aligned}
& \left\langle y_{j, G}\right\rangle^{E E}=\frac{1}{t_{E E}} \int_{0}^{t_{E E}} y_{j, G}\left(t, L_{R e g}\right) d t \\
& \left\langle y_{j, G}\right\rangle^{E L}=\frac{1}{t_{E L}} \int_{t_{E E}}^{t_{E E}+t_{E L}} y_{j, G}\left(t, L_{R e g}\right) d t \\
& y_{j, G}^{0, R}=\frac{G_{m, t o t}}{G_{m, t o t}+G_{m, L}}\left\langle y_{j, G}\right\rangle^{E E}+\frac{G_{m, L}}{G_{m, t o t}+G_{m, L}}\left\langle y_{j, G}\right\rangle^{E L}
\end{aligned}
$$


siendo $\left\langle y_{j, G}\right\rangle^{E E}$ y $\left\langle y_{j, G}\right\rangle^{E L}$, el promedio temporal de las fracciones molares en el gas de salida de la región de enfriamiento del sólido y limpieza, respectivamente.

\subsubsection{Pérdida de carga}

Al igual que para los sistemas de inversión de flujo, para el cálculo de la pérdida de carga se consideró que el régimen de flujo en cada canal corresponde al régimen laminar. Luego, la caída de presión se calcula a partir de la Ec. (3.19), evaluando las propiedades de la corriente a la temperatura media espacial (y temporal en el regenerador) de cada región/equipo.

\subsubsection{Contenido de VOCs a la salida del sistema}

La salida global del sistema corresponde con la salida de la región de calentamiento del regenerador. Luego, para conocer el contenido de VOCs en la corriente efluente:

$$
\begin{aligned}
& \left\langle y_{j, G}\right\rangle^{E C}=\frac{1}{t_{E C}} \int_{t_{E E}+t_{E L}}^{t_{\text {ciclo }}} y_{j, G}\left(t, L_{R e g}\right) d t \\
& \left\langle y_{\text {VOCs }}\right\rangle=\sum_{j=1}^{3}\left\langle y_{j, G}\right\rangle^{E C}
\end{aligned}
$$

donde $\left\langle y_{\text {VOCS }}\right\rangle$, representa la fracción molar total de VOCs a la salida del sistema e $\left\langle y_{j, G}\right\rangle^{E C}$ es la fracción molar de cada componente a la salida de la región de calentamiento del sólido.

\subsubsection{Discusión del modelo}

El modelo propuesto para ambos equipos en el sistema regenerador-reactor es, en cuanto a las suposiciones realizadas, equivalente al utilizado para los 
sistemas de inversión de flujo. De hecho, si se parte de los balances dados por las expresiones (3.9) a (3.16), se pueden obtener las ecuaciones de balances para el regenerador y para el reactor, eliminando adecuadamente los términos que no contribuyen en cada caso (por ejemplo, los términos de reacción química y transferencia de materia entre el sólido y el gas para el regenerador). Por lo tanto, la discusión realizada previamente para el modelo de los sistemas de inversión también será válida para estos modelos, inclusive la evaluación sobre la posible distribución no uniforme de flujo o la conductividad térmica en sentido angular y consecuentemente radial (aunque para esto último, ya no será válida la idea que el sistema se vería beneficiado por la conducción angular, tendiendo al comportamiento del sistema en contra-corriente, como fuera enunciado para el sistema de inversión de flujo rotativo).

Recordando que se han ignorado los términos de acumulación en el reactor, cabe preguntarse si su inclusión serían las únicas modificaciones necesarias si se quisiera obtener la evolución dinámica completa del sistema, ya sea previa al css o entre un css dado y otro frente a variaciones en las condiciones operativas. Se puede razonar que, en tales circunstancias, el modelo utilizado debería brindar la variación continua que sufre la temperatura (y eventualmente la composición) a la entrada al reactor mientras el regenerador entra en régimen. Para ello no sería suficiente la inclusión de los términos de acumulación en el reactor como única modificación, sino que también se debería conocer la variación temporal de las variables en todos los canales y no solo en una posición dada. Es decir, el modelo debería ser capaz de contemplar la diferencia que se daría en los canales ubicados en diferentes posiciones angulares cuando el regenerador operase fuera del css (aun cuando en cada canal se utilice un modelo unidimensional). Luego, la temperatura y concentración de VOCs a la entrada al reactor se obtendrian de un promedio sobre la sección transversal del regenerador a la salida de las regiones de enfriamiento y limpieza, para cada instante.

Para finalizar, cabe resaltar que el modelo presentado aquí para el estudio del regenerador, es equivalente al propuesto por Willmott (2002), con la excepción de que en el último no se contempla la existencia de la región de limpieza. 


\subsection{Aproximación de los sistemas de inversión de flujo para bajos tiempos de ciclo: reactor en contra-corriente}

Cuando se opera un reactor de inversión de flujo utilizando tiempos de ciclos muy pequeños para la longitud del equipo (pero aún elevado respecto del tiempo de residencia del gas en el canal), la temperatura del sólido prácticamente no variará, como consecuencia de la inercia térmica del mismo, mientras que la temperatura del gas oscilará alrededor de la anterior. En estos casos, la operación del reactor se aproxima a la de un reactor hipotético donde el gas circule en contra-corriente, separado por una pared sólida con la que intercambiará materia (si corresponde) y energía, siendo la temperatura del sólido uniforme en la sección transversal, pero no la concentración de reactivos en su superficie (Eigenberger y Nieken, 1994). Una de las representaciones esquemáticas típicas para este reactor en contra-corriente (RCC) se muestra en la Figura 3.4, en donde se ha considerado que la corriente del gas a tratar se divide en dos sub-corrientes que se alimentan en posiciones y sentidos opuestos.

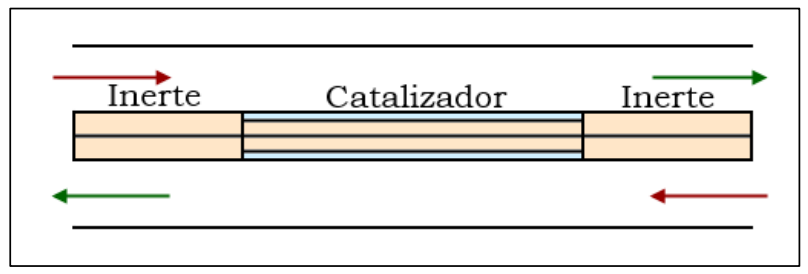

Figura 3.4: reactor hipotético con el gas en contra-corriente.

Si bien este esquema carece de importancia práctica, ha sido estudiado por varios autores y se conoce también como "modelo de altas frecuencias de inversión" (HSFM, de sus siglas en inglés). Esto se debe a que permite predecir algunos patrones de comportamiento del sistema, pero con un esfuerzo computacional significativamente menor, como consecuencia de la operación en estado estacionario. De hecho, permite arribar a una expresión analítica de la temperatura máxima en el lecho (a través de una función implícita), pero la misma está limitada a situaciones en donde solo existe una única reacción, no hay resistencia a la transferencia de energía en la interfase sólido-gas y la cinética es lineal (Matros y Bunimovich, 1996) o cuando la cinética es de tipo 
potencial y, en adición, no hay resistencia a la transferencia de materia en el interior de los poros del catalizador (Pinjala y col., 1988). Sin embargo, cuando la expresión cinética es más compleja, existen efectos difusionales en el interior de los poros o existen múltiples reacciones, no es posible encontrar expresiones similares.

Uno de los aspectos más interesantes de este modelo simplificado es que permite establecer una cota mínima para el volumen requerido del equipo de inversión de flujo, aspecto que se abordará con mayor detalle en el Capítulo 7.

Los balances necesarios para la simulación se pueden obtener desde los balances ya planteados para los sistemas de inversión de flujo, debiendo diferenciar la dirección de la corriente, lo cual se hace aquí mediante los símbolos “ $\rightarrow$ ” “ “ $\leftleftarrows$ " sobre cada variable (todas las variables poseen el mismo significado ya dado en la Sección 3.3). De esta manera, se puede plantear:

Balance de energía para la fase sólida (región catalitica):

$$
\vec{h}_{\text {mod,C }}\left(\vec{T}_{G}-T_{S}\right)+\overleftarrow{h}_{\text {mod,C }}\left(\overleftarrow{T}_{G}-T_{S}\right)+\ell \sum_{j=1}^{3}\left(-\Delta H_{j}^{\#}\right)\left(\vec{r}_{j}^{\text {ef }}+\overleftarrow{r}_{j}^{\text {ef }}\right)=0
$$

Balance de energía en la fase gaseosa de la sección superior (región catalitica):

$$
G_{C} \overrightarrow{c_{p G}} \frac{d \vec{T}_{G}}{d z}=-a_{v, C} \vec{h}_{m o d, C}\left(\vec{T}_{G}-T_{S}\right)
$$

Balance de energía en la fase gaseosa de la sección inferior (región catalitica):

$$
G_{C} \overleftarrow{c_{p G}} \frac{d \overleftarrow{T_{G}}}{d z}=+a_{v, C} \overleftarrow{h}_{m o d, C}\left(\overleftarrow{T}_{G}-T_{S}\right)
$$


Balance de materia por componente en la fase gaseosa de la sección superior (región catalitica):

$$
\frac{G_{C}}{M_{\text {aire }}} \frac{d \overrightarrow{y_{j, G}}}{d z}=-a_{v, C} \vec{k}_{m, j, C} \vec{C}_{T}\left(\vec{y}_{j, G}-\vec{y}_{j, S}\right) \quad j=1 . .3
$$

Balance de materia por componente en la fase gaseosa de la sección inferior (región catalitica):

$$
\frac{G_{C}}{M_{\text {aire }}} \frac{d \overleftarrow{y_{j, G}}}{d z}=+a_{v, C}{\overleftarrow{k_{m, j, C}}}_{C_{T}}\left(\overleftarrow{y_{j, G}}-\overleftarrow{y_{j, S}}\right) \quad j=1 . .3
$$

Balance de materia por componente en la fase sólida de la sección superior (región catalitica):

$$
\vec{k}_{m, j, C} \vec{C}_{T}\left(\vec{y}_{j, G}-\vec{y}_{j, S}\right)-\ell \overrightarrow{r_{j}^{e f}}=0 \quad j=1 . .3
$$

Balance de materia por componente en la fase sólida de la sección inferior (región catalitica):

$$
\overleftarrow{k}_{m, j, C} \overleftarrow{C}_{T}\left(\overleftarrow{y}_{j, G}^{\leftarrow}-\overleftarrow{y_{j, S}}\right)-\ell{\overleftarrow{r_{j}^{e f}}}^{\overleftarrow{ }}=0 \quad j=1 . .3
$$

Para las regiones inertes, se debe eliminar el último término a la derecha de la Ec. (3.32), mientras que las Ecs. (3.33) y (3.34) no se ven alteradas y los balances de materia por componente para estas regiones no serán necesarios por encontrarse en estado estacionario.

Por otro lado, se puede reconocer la simetría del sistema respecto de la posición axial. Esto permite resolver solo una "mitad" (por ejemplo, desde la posición de alimentación de la corriente que circula por la sección superior hasta la posición central). Con ello, las condiciones de contorno quedan definidas por las condiciones de alimentación, debiendo exigirse la continuidad de las variables en el gas en ambos límites inerte-catalizador, así como en la po- 
sición central del reactor. De esta manera, el sistema descrito conforma un sistema de ecuaciones diferenciales ordinarias y algebraicas, con condiciones de borde. La estrategia de resolución adoptada se verá en el Capítulo 4.

Por último, se debe tener presente que la formulación presentada también será útil como aproximación para la operación del regenerador a bajos tiempos de ciclos, aspecto que se aborda en el Apéndice 3.3.

\subsection{Conclusiones y comentarios finales}

Se realizó una descripción detallada de las diferencias entre los dos esquemas de inversión de flujo seleccionados en el Capítulo 2. Utilizando un razonamiento sencillo en base a la descripción de la operación realizada en dicho capítulo, se enunció que la operación del sistema de inversión de flujo convencional con tiempos de ciclo muy elevados requerirá un mayor volumen (masa) de equipo comparado a operaciones con ciclos cortos. Sin embargo, el mínimo tiempo a utilizar queda limitado por dos factores: la minima vida útil admisible de las válvulas y la emisión de los VOCs residuales en las regiones de entrada de cada semiciclo.

La inclusión del esquema de inversión de flujo rotativo responde entonces a la posibilidad de minimizar la masa de inerte y catalizador, utilizando para ello tiempos de ciclos más pequeños que en el esquema convencional. Esto es posible gracias a la presencia de regiones destinadas a la limpieza (Figura 3.1), que evitan el problema de emisión de los VOCs remanentes (inclusive en las cañerías). Como contrapartida, el esquema resulta más complejo. Como fuera mencionado en la Sección 3.1, la complejidad del sistema se podría reducir parcialmente utilizando aire proveniente del ambiente, en lugar de la corriente de salida de la región de reacción. Sin embargo, esto requeriría de la instalación de otro soplador.

Se describió el esquema del sistema regenerador-reactor (Figura 3.2), el cual también posee una región de limpieza, con la misma finalidad que en el sistema de inversión de flujo rotativo.

En la Sección 3.2 se presentó el sistema reactivo en detalle, donde se pudo apreciar que aparece un tercer componente, como resultado de la oxidación parcial del etanol. La presencia de expresiones cinéticas de tipo LHHW, con un 
término de inhibición común donde participan las concentraciones de dos componentes, presenta un grado de complejidad que deberá ser tenido en cuenta en la resolución de las ecuaciones de balances. Esto último será presentado en el Capítulo 4, donde se abordan todas las cuestiones relativas a la resolución de los modelos. En la Sección 3.2 también se indicaron los materiales a considerar, tanto para las regiones inertes de los equipos (cordierita) como para el de las catalíticas.

Por último, se plantearon todos los balances necesarios para la simulación de cada esquema, incluyendo una aproximación a tiempos de ciclo pequeños (RCC). Se discutieron las principales simplificaciones realizadas en los modelos planteados, haciendo una breve revisión de los principales trabajos enfocados en la simulación con modelos de diferentes grados de complejidad. De dicha discusión, se concluyó que las simplificaciones adoptadas serán adecuadas para el sistema a analizar en el presente trabajo. 
- 70 - 


\section{Apéndice 3.1: Propiedades físicas y coeficientes de transferencia de materia y energia}

Teniendo presente el bajo contenido de VOCs en la corriente a tratar, la misma se consideró como aire puro a los fines del cálculo de las propiedades físicas. Excepto para la estimación de la densidad y de los coeficientes de difusión moleculares, para todas las demás propiedades físicas, se tomaron los datos y correlaciones de Poling y col. (2008).

\section{Densidad}

Para el cálculo de la densidad, se consideró en forma adicional que el comportamiento de la corriente se ajusta al comportamiento de gas ideal. Esto se puede justificar fácilmente si se considera que la presión reducida del aire a presión atmosférica es tan solo 0.027 .

\section{Viscosidad}

Para la viscosidad, la expresión utilizada es:

$$
\mu_{G}=1.42510^{-6} \frac{T^{0.5039}}{1+108.3 / T}
$$

donde, $T$ es la temperatura del gas en $\mathrm{K} \mathrm{y} \mu_{G}$ la viscosidad en [Pa s]. El rango de aplicabilidad de la expresión es 80-2000 K.

\section{Calor especifico}

Para el calor específico, la expresión utilizada es:

$$
c_{p G}=A+B\left[\frac{C / T}{\sinh (C / T)}\right]^{2}+D\left[\frac{E / T}{\sinh (E / T)}\right]^{2}
$$


siendo

$$
\begin{aligned}
& A=28958 / M_{\text {aire }} \\
& B=9390 / M_{\text {aire }} \\
& C=3012 \\
& D=7580 / M_{\text {aire }} \\
& E=1484
\end{aligned}
$$

y $T$ la temperatura del gas en $\mathrm{K}, M_{\text {aire }}$ el peso molecular del aire en $\mathrm{kg} / \mathrm{kmol} \mathrm{y}$ $c_{p G}$ el calor específico en $[\mathrm{J} /(\mathrm{kg} \mathrm{K})]$. El rango de aplicabilidad de la expresión es $50-1500 \mathrm{~K}$.

\section{Calor especifico medio}

Como se desprende de la expresión (A3.1.2), el calor específico de la corriente no depende de la composición de los VOCs, consecuencia directa de haber considerado las propiedades fisicas de la corriente como las del aire puro. Bajo estas circunstancias, puede ser útil disponer de una expresión para el calor específico medio entre una temperatura $T$ y un valor $T_{\text {ref }}$, como una función de la temperatura T. Es decir:

$$
\bar{c}_{p G}\left(T, T_{r e f}\right)=\frac{1}{T-T_{r e f}} \int_{T_{r e f}}^{T} c_{p G}(u) d u
$$

donde $\bar{c}_{p G}\left(T, T_{r e f}\right)$ es el calor específico medio en $[\mathrm{J} /(\mathrm{kg} \mathrm{K})]$ entre $T$ y $T_{\text {ref }}$, siendo $T_{\text {ref }}$ una temperatura de referencia, arbitrariamente elegida.

Integrando ${ }^{1}$ luego la expresión (A3.1.2), se obtiene:

\footnotetext{
${ }^{1}$ Los términos hiperbólicos se integran fácilmente mediante un cambio de variables del tipo $v=C / T$ o $v=E / T$, según corresponda.
} 


$$
\bar{c}_{p G}\left(T, T_{r e f}\right)=A+\frac{B C \operatorname{coth}(C / T)-D E \tanh (E / T)-G\left(T_{r e f}\right)}{T-T_{r e f}}
$$

siendo, $G\left(T_{\text {ref }}\right)=B C \operatorname{coth}\left(C / T_{\text {ref }}\right)-D E \tanh \left(E / T_{\text {ref }}\right), T$ es la temperatura del gas en $\mathrm{K}, M_{\text {aire }}$ el peso molecular del aire en $\mathrm{kg} / \mathrm{kmol}$.

\section{Conductividad térmica}

Para la conductividad térmica, que se utiliza en las correlaciones para los coeficientes de transferencia (ver más adelante en este apéndice), la expresión corresponde a:

$$
\lambda_{G}=3.141710^{-4} \frac{T^{0.7786}}{1-0.7116 / T+2121.7 / T^{2}}
$$

donde, $T$ es la temperatura del gas en $\mathrm{K}$ y $\lambda_{G}$ la conductividad en $[\mathrm{W} /(\mathrm{m} \mathrm{K})]$. El rango de aplicabilidad de la expresión es 70-2000 K.

\section{Coeficientes de difusión molecular}

Los coeficientes de difusión molecular se evaluaron a partir de la correlación propuesta por Fuller y col. (1966), considerando que cada componente difunde en aire, como si fuera un problema de difusividad binaria. Esta expresión viene dada por:

$$
D_{m, j}=10^{-7} \frac{T^{1.75}\left(M_{j}^{-1}+M_{\text {aire }}^{-1}\right)^{1 / 2}}{P\left(v_{j}^{1 / 3}+v_{\text {aire }}^{1 / 3}\right)^{2}}
$$


donde la temperatura $T$ debe ingresarse en $\mathrm{K}, M_{j}$ y $M_{\text {aire }}$ son los pesos moleculares del componente " $j$ " y del aire, respectivamente, ambos en g/mol, $P$ es la presión en atm, $v_{j}$ y $v_{\text {aire }}$ son los volúmenes de difusión del componente “j” y del aire (los cuales se listan en la Tabla A3.1.1), respectivamente, ambos en $\left[\mathrm{cm}^{3} / \mathrm{mol}^{-1}\right] . D_{m, j}$ es el coeficiente de difusión molecular del componente “ $j$ ” en aire, dado en $\left[\mathrm{m}^{2} / \mathrm{s}^{-1}\right]$.

Tabla A3.1.1: volúmenes de difusión.

\begin{tabular}{ccccc}
\hline & $\begin{array}{c}\text { Acetato de etilo } \\
\text { (1) }\end{array}$ & $\begin{array}{c}\text { Etanol } \\
\text { (2) }\end{array}$ & $\begin{array}{c}\text { Acetaldehido } \\
\text { (3) }\end{array}$ & Aire \\
\hline$v_{\boldsymbol{j}}\left[\mathbf{c m}^{3} / \mathbf{m o l}\right]$ & 65.73 & 39.08 & 37.66 & 20.1 \\
\hline
\end{tabular}

\section{Entalpias de combustión}

Para la evaluación de las entalpías de combustión a la temperatura de reacción, se tomaron los valores de las mismas en el Estado de Gas Ideal, referidas a 1 atm y $298 \mathrm{~K}$, y se corrigieron por temperatura mediante la integración de la capacidad calorífica a presión constante de las sustancias involucradas en la reacción. Para esto último, se consideró una expresión lineal de la capacidad calorífica molar de cada componente con la temperatura, propuesta por Campesi (2012), de la forma:

$$
c_{p, j}=a_{j}+b_{j} T
$$

Luego, considerando que las reacciones de combustión completa corresponden a:

$$
\begin{aligned}
& \mathrm{C}_{4} \mathrm{H}_{8} \mathrm{O}_{2}+5 \mathrm{O}_{2} \rightarrow 4 \mathrm{CO}_{2}+4 \mathrm{H}_{2} \mathrm{O} \\
& \mathrm{C}_{2} \mathrm{H}_{6} \mathrm{O}+3 \mathrm{O}_{2} \rightarrow 2 \mathrm{CO}_{2}+3 \mathrm{H}_{2} \mathrm{O} \\
& \mathrm{C}_{2} \mathrm{H}_{4} \mathrm{O}+5 / 2 \mathrm{O}_{2} \rightarrow 2 \mathrm{CO}_{2}+2 \mathrm{H}_{2} \mathrm{O}
\end{aligned}
$$


Nombrando los componentes de acuerdo con: 1 - acetato de etilo, 2 - etanol, 3 - acetaldehído, 4 - oxígeno, 5 - dióxido de carbono, 6 - agua, y definiendo la matriz de coeficientes estequiométricos (las filas corresponden a las sustancias y las columnas a las reacciones):

$$
\underline{\mathbf{a}}=\left[\begin{array}{ccc}
-1 & 0 & 0 \\
0 & -1 & 0 \\
0 & 0 & -1 \\
-5 & -3 & -5 / 2 \\
4 & 2 & 2 \\
4 & 3 & 2
\end{array}\right]
$$

Se puede calcular la entalpía de combustión de cada componente clave (1 a 3) de:

$$
\boldsymbol{\Delta} \mathbf{H}^{\#}=\boldsymbol{\Delta} \mathbf{H}^{0}+\underline{\mathbf{a}}^{T}\left[\mathbf{a}+\mathbf{b}\left(\frac{T+T_{r e f}}{2}\right)\right]\left(T-T_{r e f}\right)
$$

siendo, $\boldsymbol{\Delta} \mathbf{H}^{\#}$ un vector columna con las entalpías de combustión de los componentes claves a la temperatura $T$ (en K), $\boldsymbol{\Delta} \mathbf{H}^{0}$ el vector columna con las entalpías de formación de los componentes claves en el Estado de Gas Ideal, a y b vectores columna con los coeficientes correspondientes a las capacidades caloríficas de cada componente dadas por la expresión (A3.1.7) y $T_{\text {ref }}$ la temperatura de referencia (298 $\mathrm{K}$ en este caso). Las unidades de $\boldsymbol{\Delta} \mathbf{H}^{\#}$ y $\boldsymbol{\Delta} \mathbf{H}^{0}$ son $[\mathrm{J} / \mathrm{mol}]$. Los valores de $\Delta H_{j}^{0}, a_{j}$ y $b_{j}$ se dan en la Tabla A3.1.2. 
Tabla A3.1.2: coeficientes para el cálculo de las entalpías de combustión.

\begin{tabular}{ccccc}
\hline Componente & $\begin{array}{c}\Delta \mathbf{H}_{\mathbf{j}}^{\mathbf{0}} \\
{[\mathbf{J} / \mathbf{m o l}]}\end{array}$ & $\begin{array}{c}\boldsymbol{a}_{j} \\
{[\mathbf{J} /(\mathbf{m o l ~ K})]}\end{array}$ & $\begin{array}{c}\boldsymbol{b}_{j} \\
{\left[\mathbf{J} /\left(\mathbf{m o l ~ K} \mathbf{~ K}^{2}\right)\right]}\end{array}$ \\
\hline 1 & $-2.06110^{6}$ & $5.32410^{1}$ & $2.15410^{-1}$ \\
\hline 2 & $-1.23510^{6}$ & $3.44510^{1}$ & $1.12110^{-1}$ \\
\hline 3 & $-1.10510^{6}$ & $3.14110^{1}$ & $9.00010^{-2}$ \\
\hline 4 & --- & $2.63010^{1}$ & $1.00010^{-2}$ \\
\hline 6 & -- & $3.18810^{1}$ & $2.50010^{-2}$ \\
\hline 6 & -- & $2.96710^{1}$ & $1.10010^{-1}$ \\
\hline
\end{tabular}

\section{Coeficientes de transferencia}

Para el cálculo de los coeficientes de transferencia de materia y energía, se utilizó la correlación propuesta por Barreto (2008) para el número de Sherwood, con la condición de concentración constante. Dicha correlación adopta la forma:

$$
S h_{j}=S h_{\infty}+\frac{5.4 \delta_{j} P e_{j}}{\left(12 \delta_{j}+0.519 P e_{j}^{3 / 2}\right)^{1 / 3}}
$$

donde $S h_{\infty}$ es el valor que alcanza el Sh para cualquier componente cuando los perfiles de velocidad y concentración se encuentran desarrollados (valor asintótico), $P e_{j}$ es el número de Peclet transversal para el componente “ $j$ ” y $\delta_{j}$ es una función del número de Schmidt, $S c_{j}$, y la forma del canal. Estos parámetros se definen como:

$$
P e_{j}=\left(\frac{\varepsilon_{L}}{a_{v}}\right)^{2} \frac{G / \varepsilon_{L}}{\rho_{G} D_{m, j} L_{M}}
$$




$$
\begin{aligned}
& \delta_{j}=\left[\left(\frac{f \mathrm{Re}}{100}\right)^{4} \beta_{\infty}^{12}+\left(\frac{1}{\beta_{0}^{6}}+\frac{S c_{j}}{\beta_{1}^{6}}\right)^{-2}\right]^{1 / 12} \\
& S c_{j}=\frac{\mu_{G}}{\rho_{G} D_{m, j}}
\end{aligned}
$$

donde, $L_{M}$ representa la longitud de cada módulo del monolito y $\beta_{0}, \beta_{1}$ y $\beta_{\infty}$ son constantes independientes de la forma del canal. Finalmente, a partir de la definición del número de Sherwood y considerando que el diámetro hidráulico del canal corresponde a $4\left(\varepsilon / a_{v}\right)$, el coeficiente de transferencia de materia, $k_{m, j}$, se calcula de:

$$
k_{m, j}=\frac{S h_{j} D_{m, j}}{4\left(\varepsilon_{L} / a_{v}\right)}
$$

Los valores de cada uno de los parámetros necesarios para el cálculo del coeficiente de transferencia de materia se listan en la Tabla A3.1.3. Para aquellos valores que dependen de la geometría del canal, solo se da el valor correspondiente a un canal perfectamente cuadrado.

Tabla A3.1.3: parámetros para el cálculo de los coeficientes de transferencia.

\begin{tabular}{ccccccc}
\hline $\begin{array}{c}\boldsymbol{f} \mathbf{R e}) \\
\text { (canal cuadrado) }\end{array}$ & $\begin{array}{c}\boldsymbol{S h}_{\infty} \\
\text { (canal cuadrado) }\end{array}$ & $\boldsymbol{\beta}_{\mathbf{0}}$ & $\boldsymbol{\beta}_{\mathbf{1}}$ & $\boldsymbol{\beta}_{\infty}$ & $\boldsymbol{L}_{\boldsymbol{M}}$ \\
\hline 14.2 & 2.977 & 0.5642 & 0.3392 & 0.4273 & 0.25 \\
\hline
\end{tabular}

En cuanto al coeficiente de transferencia de energía, considerando la similitud entre este proceso y el de transferencia de materia, se puede utilizar la expresión (A3.1.9) para el cálculo del primero, reemplazando el Sh por el número de Nusselt, $\mathrm{Nu}$, el $S c_{j}$ por el número de Prandtl, $\mathrm{Pr}$, y el Pe por el número de Peclet térmico, $P e_{T}$. De esta manera, se tiene: 


$$
\begin{aligned}
& \operatorname{Pr}=\frac{\mu_{G} c_{p G}}{\lambda_{G}} \\
& P e_{T}=\left(\frac{\varepsilon_{L}}{a_{v}}\right)^{2} \frac{c_{p G} G / \varepsilon_{L}}{\rho_{G} L_{M} \lambda_{G}} \\
& h=\frac{N u \lambda_{G}}{4\left(\varepsilon_{L} / a_{v}\right)}
\end{aligned}
$$




\section{Apéndice 3.2: Parámetros geométricos del sistema}

La caracterización de las matrices de monolitos se realiza usualmente mediante el conocimiento de: la forma del canal, el número de celdas totales en una dada sección transversal, $N_{C}$, el espesor de tabique, $\delta_{T}$, y el radio de curvatura, $R_{C}$ (ver Figura A3.2.1). Adicionalmente, para aquellas matrices con un depósito de material catalítico, se deberá conocer el espesor del depósito, $\delta_{\text {dep }}$.

En la Figura A3.2.1 se muestran las variables principales para una matriz cuadrada que permiten el cálculo de los parámetros geométricos necesarios para el modelo, esencialmente $\varepsilon_{L}, a_{v} \mathrm{y} \ell$. En la misma se delimitó con líneas punteadas una celda del monolito, señalando qué representa cada una de las variables antes mencionadas y definiendo la longitud de la celda, $L_{C}$.

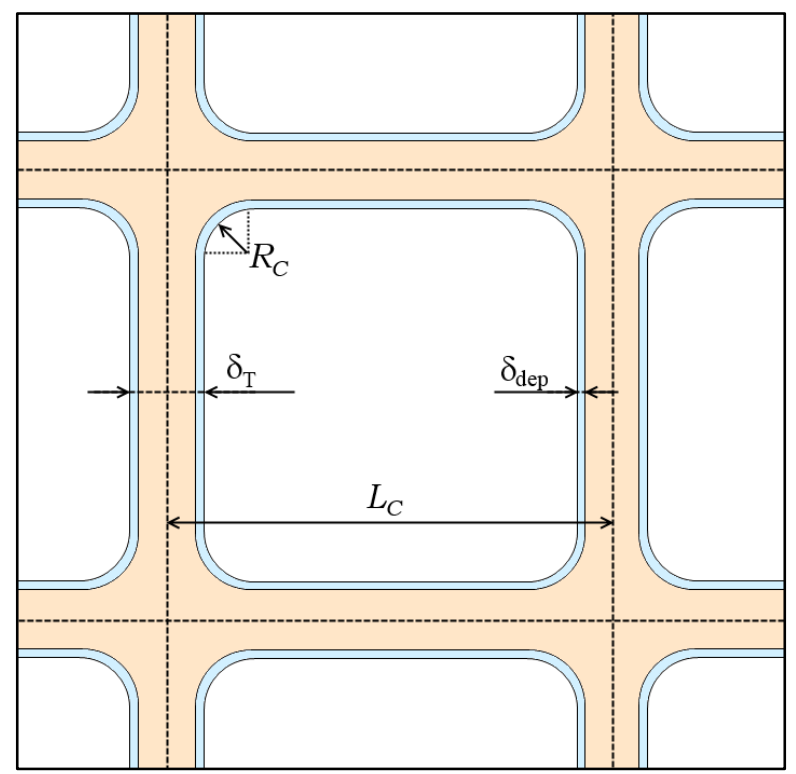

Figura A3.2.1: representación de una celda de monolito.

Luego, a partir de relaciones geométricas se obtiene:

$$
L_{C}=N_{C}^{-1 / 2}
$$




$$
\begin{aligned}
& a_{v}=\frac{4\left(L_{C}-\delta_{T}\right)-4\left(2 R_{C}\right)+4\left(\frac{\pi R_{C}}{2}\right)}{L_{C}^{2}}=\frac{4}{L_{C}}\left[\left(1-\frac{\delta_{T}}{L_{C}}\right)-\frac{1}{2}(4-\pi) \frac{R_{C}}{L_{C}}\right] \\
& \varepsilon_{L}=\frac{\left(L_{C}-\delta_{T}\right)^{2}-4 R_{C}^{2}+\pi R_{C}^{2}}{L_{C}^{2}}=\left(1-\frac{\delta_{T}}{L_{C}}\right)^{2}-(4-\pi)\left(\frac{R_{C}}{L_{C}}\right)^{2}
\end{aligned}
$$

Finalmente, para el cálculo de la longitud característica del catalizador, $\ell$, suponiendo que el espesor de depósito catalítico es completamente uniforme y recordando que $\varepsilon_{L}^{0}$ representa la porosidad que tendría el monolito previo a haber depositado el catalizador, se tiene:

$$
\begin{aligned}
\varepsilon_{L}^{0} & =\frac{\left[L_{C}-\left(\delta_{T}-2 \delta_{d e p}\right)\right]^{2}-\left[4\left(R_{C}+\delta_{d e p}\right)^{2}+\pi\left(R_{C}+\delta_{d e p}\right)^{2}\right]}{L_{C}^{2}} \\
& =\left(1-\frac{\left(\delta_{T}-2 \delta_{d e p}\right)}{L_{C}}\right)^{2}-(4-\pi)\left(\frac{R_{C}+\delta_{d e p}}{L_{C}}\right)^{2} \\
\ell & =\frac{\varepsilon_{L}^{0}-\varepsilon_{L}}{a_{v}}
\end{aligned}
$$

Por una cuestión de generalidad, se ha incluido en la formulación el radio de curvatura, $R_{C}$. No obstante, el mismo se consideró nulo en todos los casos, entendiendo que su efecto no será significativo ni modificará cualitativamente los resultados alcanzados en el presente estudio. En adición, cabe resaltar que para el presente caso resulta en general $\ell=\delta_{\text {dep }}$. 


\section{Apéndice 3.3: Similitud entre el regenerador y un intercambiador en contra-corriente para bajos tiempos de ciclo}

Tal como sucede en el esquema de inversión de flujo, cuando el regenerador se opera con un tiempo de ciclo muy pequeño (pero aún mayores que el tiempo de residencia del gas), la temperatura de la fase sólida no varía apreciablemente en el tiempo (y consecuentemente tampoco lo hará entre regiones, sino solo en el sentido axial). En tal circunstancia, el comportamiento térmico de este equipo se aproximará al de un intercambiador de calor en contra-corriente (despreciando la influencia de la región de limpieza sobre los perfiles de temperatura). Como fuera enunciado en la Sección 3.5, se pueden utilizar los balances de energía presentados en la aproximación del reactor en contra-corriente, eliminando los términos de reacción química. Es decir, manteniendo la nomenclatura utilizada en dicho caso para diferenciar la corriente que circula por la región de enfriamiento del sólido, $\vec{T}_{G}$, de aquella que circula por la región de calentamiento, $\overleftarrow{T}_{G}$, se tiene:

Balance de energía para la fase sólida:

$$
\vec{h}_{\text {mod }}\left(\vec{T}_{G}-T_{S}\right)+\overleftarrow{h}_{\text {mod }}\left(\overleftarrow{T}_{G}-T_{S}\right)=0
$$

Balance de energía en la fase gaseosa (región de enfriamiento):

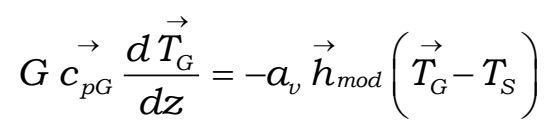

Balance de energía en la fase gaseosa (región de calentamiento):

$$
G \underset{c_{p G}}{\leftarrow} \frac{d T_{G}}{d z}=+a_{v} \overleftarrow{h}_{m o d}\left(\overleftarrow{T}_{G}-T_{S}\right)
$$


Explicitando $T_{S}$ de la Ec. (A3.3.1), se tiene:

$$
\begin{aligned}
& T_{S}=\frac{\vec{h}_{\text {mod }} \vec{T}_{G}+\overleftarrow{h}_{\text {mod }} \overleftarrow{T}_{G}}{\vec{h}_{\text {mod }}+\overleftarrow{h}_{\text {mod }}} \\
& -a_{v} \vec{h}_{\text {mod }}\left(\vec{T}_{G}-T_{S}\right)=a_{v} \overleftarrow{h}_{\text {mod }}\left(\overleftarrow{T}_{G}-T_{S}\right)=a_{v} \frac{\vec{h}_{\text {mod }} \overleftarrow{h}_{\text {mod }}}{\vec{h}_{\text {mod }}+\overleftarrow{h}_{\text {mod }}}\left(\overleftarrow{T}_{G}-\vec{T}_{G}\right)
\end{aligned}
$$

Con la Ec. (A3.3.5) en (A3.3.2) y (A3.3.3), se puede corroborar que si $c_{p G}$ resulta constante, la diferencia $\stackrel{\leftarrow}{T_{G}}-\vec{T}_{G}=\Delta T^{R}$ permanecerá constante a lo largo del regenerador (siendo igual al salto térmico en el reactor).

Manteniendo tal suposición sobre el calor específico del gas (la variación que ofrece $c_{p G}$ es muy baja), la relación $\frac{\vec{h}_{\text {mod }} \overleftarrow{h}_{\text {mod }}}{\vec{h}_{\text {mod }}+\overleftarrow{h}_{\text {mod }}}$ vendrá dada por

$$
\frac{\vec{h}_{\text {mod }} \overleftarrow{h}_{\text {mod }}}{\vec{h}_{\text {mod }}+\overleftarrow{h}_{\text {mod }}} \equiv\left[\frac{1}{h_{\text {mod }}\left(\vec{T}_{G}\right)}+\frac{1}{h_{\text {mod }}\left(\vec{T}_{G}+\Delta T^{R}\right)}\right]^{-1} \approx \frac{1}{2} h_{\text {mod }}\left(\left(T_{G}^{0}+T_{G}^{0, R}\right) / 2\right)
$$

donde, a fines de simplificar la formulación, se consideró un valor constante para el coeficiente de transferencia de energía (evaluándolo a la temperatura promedio de la corriente) y tomando $h_{\text {mod }}\left(\vec{T}_{G}+\Delta T^{R}\right) \approx h_{\text {mod }}\left(\vec{T}_{G}\right)$.

Incorporando la Ec. (A3.3.6) en la (A3.3.3) e integrando entre $\vec{T}_{G}(0)=T_{G}^{0}$ y $\vec{T}_{G}\left(L_{R e g}\right)=T_{G}^{0, R}$ (siendo $L_{R e g}$ la longitud del regenerador):

$$
Q=G_{m, t o t} c_{p G}\left(T_{G}^{0, R}-T_{G}^{0}\right)=\frac{a_{v} V_{R e g}}{2} \frac{h_{\text {mod }}\left(\left(T_{G}^{0}+T_{G}^{0, R}\right) / 2\right)}{2} \Delta T^{R}
$$


en donde $G_{m, t o t}$ es el caudal másico total de la corriente a tratar, $V_{\text {Reg }}$ el volumen total del regenerador y $Q$ la energía intercambiada entre las corrientes. Además, se ha considerado $G / L_{\text {Reg }}=G_{m, t o t} /\left(V_{R e g} / 2\right)$, para lo cual se tuvo en cuenta que, en ausencia de la región de limpieza, las secciones de flujo para ambas corrientes serán iguales entre sí (e igual a la mitad de la sección total).

Finalmente, a partir de la ecuación de diseño típica para un intercambiador de superficie en contra-corriente:

$$
Q=U A \Delta T_{m l}
$$

siendo $U$ el coeficiente global de transferencia de energía, $A$ el área de intercambio y $\Delta T_{m l}$ el salto térmico medio logarítmico (igual en este caso a $\Delta T^{R}$ ), se puede encontrar la equivalencia entre el factor $U A$ y las propiedades del regenerador. Es decir, igualando la Ec. (A3.3.8) con la expresión derecha de la Ec. (A3.3.7):

$$
U A=\frac{h_{\text {mod }}\left(\left(T_{G}^{0}+T_{G}^{0, R}\right) / 2\right)}{2} \frac{a_{v} V_{R e g}}{2}
$$

El conjunto de ecuaciones presentado hasta aquí (Ecs. A3.3.4 a A3.3.9) permite establecer un vínculo con un intercambiador de calor de superficie. Luego, suponiendo conocido el salto térmico en el reactor, cuando el tiempo de ciclo es pequeño, permite estimar la temperatura de entrada al reactor si el volumen de equipo está definido o, dado un valor de dicha temperatura, estimar el volumen requerido para alcanzar la misma. 
- 84 - 


\section{Capítulo 04}

\section{- Implementación numérica}

- Velocidad de reacción efectiva

- Determinación de caudales de limpieza

- Algoritmo interno

- Algoritmo externo

- Algoritmo para el reactor en contra-corriente 

Definidos los modelos a utilizar para la simulación de cada sistema, resta abordar la resolución de las ecuaciones resultantes a fin de determinar el css. Aquí se puede reconocer una doble problemática: por un lado, para un dado ciclo, se deberá resolver un conjunto de ecuaciones diferenciales parciales, mediante un método numérico adecuado. Por el otro, una vez resuelto el ciclo en cuestión, se deberá establecer un algoritmo de búsqueda para la determinación del css.

Otro aspecto relativo a la implementación del modelo en un simulador, es el cálculo de las velocidades de reacción efectiva de cada especie, lo que no resulta trivial para un sistema con más de una reacción cuyas expresiones cinéticas se encuentran vinculadas entre sí.

Considerando lo expuesto en los párrafos anteriores, en este capítulo se describe en primer lugar el procedimiento adoptado para la evaluación de las velocidades de reacción (consumo) de cada especie. En segundo lugar, se aborda la determinación del caudal de limpieza (ver Capítulo 3), tanto para el esquema de inversión de flujo rotativo como para el regenerador. Luego, se presentan las estrategias de cálculo utilizadas para las dos tareas descritas inicialmente. A fin de reconocer la diferente entidad de las mismas, se designará como "algoritmo interno" a la estrategia adoptada para la simulación de un dado ciclo y como "algoritmo externo" a la búsqueda del css, esto último supuesto que se dispone de la información referente al comportamiento del sistema en los ciclos inmediatos anteriores. Adicionalmente, se presenta un análisis sobre el desempeño de los algoritmos propuestos, particularizado para el sistema de inversión de flujo (esquema convencional). Por último, se describen también los lineamientos básicos de la implementación para la aproximación de ese sistema a tiempos de ciclos muy pequeños a través del reactor en contra-corriente $(\mathrm{RCC})$ presentado en el Capítulo 3.

\subsection{Velocidad de reacción efectiva}

Como se vio en el capítulo anterior, el sistema reactivo queda descrito por tres reacciones de oxidación con expresiones cinéticas de tipo LHHW, involucrando un término de inhibición común a todas. En principio, este tipo de sistema requeriría de la integración de los balances de conservación de cada 
componente en la capa catalíticamente activa, en cada posición a lo largo del lecho, dados por:

$$
\begin{aligned}
& D_{1} \nabla^{2} C_{1}=r_{1} \\
& D_{2} \nabla^{2} C_{2}=r_{2} \\
& D_{3} \nabla^{2} C_{3}=r_{3}-r_{2}
\end{aligned}
$$

donde $C_{j}$ representa la concentración del componente “ $j$ ", $D_{j}$ el coeficiente de difusión efectivo asociado a dicho componente y las $r_{i}$ corresponden a las expresiones cinéticas informadas en el Capítulo 3. Los coeficientes de difusión efectivos de cada componente se calculan con la expresión de Bosanquet, siendo:

$$
D_{j}=\frac{\varepsilon_{C}}{\tau_{C}}\left(\frac{1}{D_{K, j}}+\frac{1}{D_{m, j}}\right)^{-1} \quad j=1 . .3
$$

$\operatorname{con} \varepsilon_{C}$ y $\tau_{C}$ la porosidad y tortuosidad del material catalítico (informadas en la Tabla 3.1), $D_{K, j}$ el coeficiente de difusión de Knudsen de la especie “ $j ” y$ $D_{m, j}$ el coeficiente de difusión molecular de la especie “j” en aire (expresión A3.1.6).

Sin embargo, se ha desarrollado una alternativa que permite evitar dicha integración, con muy poca pérdida de precisión y una clara ganancia en estabilidad y tiempo requerido para el cálculo. La misma se basa en explotar la similitud en las expresiones cinéticas (término de inhibición común), combinando los balances de forma tal que cada una de las ecuaciones resultantes quede expresada bajo una misma forma, lo cual da lugar a la aparición del balance de conservación de un componente "ficticio". Los detalles para su implementación pueden encontrarse en Campesi y col. (2012a), por lo que aquí solo se darán los lineamientos generales. 
Para el presente caso, luego de combinar adecuadamente los balances dados en (4.1) se tendrá:

$$
D_{j}^{*} \nabla^{2} C_{j}^{*}=k_{j}^{*} C_{j}^{*} / \text { In } \quad j=1 . .3
$$

donde $C_{j}^{*}$ representa la concentración del componente " $j$ ", en principio modificado (real o ficticio), $D_{j}^{*}$ el coeficiente de difusión efectivo asociado a dicho componente, $k_{j}^{*}$ el coeficiente cinético de la reacción asociada a dicho componente e In el término de inhibición común. Para este sistema reactivo, las concentraciones de los componentes " $j$ ” modificados y los parámetros $k_{j}^{*}$ y $D_{j}^{*}$ quedan definidos en términos de las concentraciones de los componentes y parámetros reales como:

$$
k_{j}^{*}=k_{j} ; \quad D_{j}^{*}=D_{j} ; \quad C_{1}^{*}=C_{1} ; \quad C_{2}^{*}=C_{2} ; \quad C_{3}^{*}=\beta C_{3}+C_{2}
$$

donde $\beta$ es un coeficiente de combinación y viene dado por $\beta=D_{3} / D_{2}-k_{3} / k_{2}$.

Teniendo en cuenta la relación entre las concentraciones de los componentes modificados y reales, el término de inhibición se puede escribir como:

$$
\text { In }=1+K_{1} C_{1}^{*}+\frac{K_{3}}{\beta}\left(C_{3}^{*}-C_{2}^{*}\right)
$$

Luego, la velocidad de consumo efectiva de cada componente modificado, $\left(r_{j}^{*}\right)^{e f}$, se calcula a través de:

$$
\left(r_{j}^{*}\right)^{e f}=\left(r_{j}^{*}\right)^{S} \eta_{j}^{*} \quad j=1 . .3
$$


donde $\left(r_{j}^{*}\right)^{S}$ es la velocidad de consumo en la superficie externa del catalizador y $\eta_{j}^{*}$ el factor de efectividad, ambos para los componentes modificados. El factor de efectividad para cada componente se aproxima utilizando la expresión correspondiente a una única reacción con cinética lineal. Así, considerando que el depósito catalítico se comporta como si se tratase de una placa plana, el factor de efectividad queda definido en términos del Módulo de Thiele para cada componente modificado, $\phi_{j}^{*}$, según:

$$
\begin{array}{ll}
\eta_{j}^{*}=\tanh \left(\phi_{j}^{*}\right) / \phi_{j}^{*} & j=1 . .3 \\
\left(\phi_{j}^{*}\right)^{2}=\frac{\ell^{2}\left(r_{j}^{*}\right)^{S}}{D_{j}^{*}\left(C_{j}^{*}\right)^{S}} \frac{1}{2 \int_{0}^{1} \frac{\operatorname{In}^{S}}{\operatorname{In}\left(Y_{j}^{*}\right)} Y_{j}^{*} d Y_{j}^{*}} & j=1 . .3
\end{array}
$$

donde $Y_{j}^{*}=C_{j}^{*} /\left(C_{j}^{*}\right)^{S}$. Para calcular el integrando en (4.7), las $Y_{j}^{*}$ se relacionan con las concentraciones de los restantes componentes " $l$ " (con $l \neq j$ ), a partir de:

$$
Y_{l}^{*}=\left(Y_{j}^{*}\right)^{m_{l}} \quad \text { con } \quad m_{l}=\left(D_{j} k_{l}\right) /\left(D_{l} k_{j}\right) \quad j=1 . .3
$$

Finalmente, la velocidad de consumo efectiva de cada componente (real), será:

$$
r_{1}^{e f}=\left(r_{1}^{*}\right)^{e f} ; \quad r_{2}^{e f}=\left(r_{2}^{*}\right)^{e f} ; \quad r_{3}^{e f}=\frac{1}{\beta}\left[\left(r_{3}^{*}\right)^{e f}-\frac{D_{3}}{D_{2}}\left(r_{2}^{*}\right)^{e f}\right]
$$

Esta aproximación ha sido comparada con la resolución rigurosa de los balances de conservación dados por las expresiones (4.1), demostrando ser muy precisa y encontrando que los mayores errores quedan sujetos al uso de 
la expresión (4.6), más que en la aproximación en sí misma. Para todas las simulaciones realizadas en este estudio, se adoptó la aproximación descrita.

\subsection{Determinación de caudales de limpieza}

En los sistemas rotativos se debe estimar el caudal utilizado para la etapa de limpieza (ver Figuras 3.1 y 3.2). Definida la fracción de la sección total destinada para ello, dicho caudal deberá ser elegido de manera tal que permita desplazar todo el volumen de gas residual (es decir, la fracción de volumen que queda sin tratar), hasta una posición dada. En el caso del sistema de inversión de flujo, dicha posición deberá corresponder a la zona en la región catalítica donde la temperatura resulte suficientemente elevada como para que la reacción se lleve a cabo. Para el sistema regenerador-reactor, se deberá desplazar la totalidad del volumen residual en el regenerador, por lo cual la posición axial corresponderá a la longitud total.

En primer lugar, resulta inmediato que si el caudal utilizado es muy pequeño, no se podrá desplazar la totalidad del volumen residual. Existe pues un valor mínimo que permitirá cumplir el objetivo propuesto y corresponde al valor para el cual el tiempo necesario para desplazar dicho volumen coincide exactamente con el tiempo de duración de la etapa. Por lo tanto, el caudal utilizado en la región de limpieza deberá ser siempre mayor o igual al mínimo.

Por otro lado, el uso de caudales muy elevados para la limpieza del canal no será recomendado, debido a que aumentará la pérdida de carga en la etapa y una vez que el volumen remanente haya sido desplazado, el tiempo restante solo contribuirá al enfriamiento del canal (efecto que deberá evitarse). En adición, si para el sistema de inversión de flujo el caudal utilizado para la limpieza fuese tal que la velocidad másica en esta etapa resultase significativamente mayor a la velocidad másica en la etapa de reacción, se desplazaría el volumen remanente pero podría no tener el tiempo de residencia necesario para reaccionar, por lo cual no se lograría cumplir el objetivo de mitigar dicha emisión.

Teniendo esto presente y suponiendo que se ha fijado la fracción utilizada para la limpieza, se puede estimar el caudal másico mínimo necesario, asumiendo que la temperatura del gas no varia en dicha etapa (esta suposición 
solo se utiliza en esta estimación y luego se libera). Luego, para el regenerador se tendrá:

$$
G_{m, L, \min }=\frac{f_{L} S_{t o t, R e g} \varepsilon_{L, I}}{t_{E L}} \int_{0}^{L_{R e g}} \rho_{G}\left(T_{G}\left(t_{E C}, z\right)\right) d z
$$

donde $G_{m, L, m i n}$ representa el caudal másico mínimo, $S_{t o t, R e g}$ la sección transversal total del regenerador y $f_{L}$ la fracción de la sección del equipo destinada para la limpieza respecto de la total, mientras que $L_{\text {Reg }}$ corresponde a la longitud total del regenerador.

Por otro lado, para el sistema de inversión de flujo rotativo, identificando la zona donde la reacción es efectivamente llevada a cabo como aquel punto del sistema, $z^{\#}$, donde la conversión total de VOCs evaluada al tiempo $t_{R}$ ha alcanzado el 99\%, se tendrá:

$$
\sum_{j=1}^{3} y_{j, G}\left(t_{R}, z^{\#}\right)=0.01 \sum_{j=1}^{3} y_{j, G}^{0}
$$

Si no existe un valor $z^{\#}$ donde se verifique (4.11) se adopta directamente la longitud total del equipo, es decir $z^{\#}=L_{e q}$ (esto solo ocurrirá cuando el nivel térmico en el lecho no resulte suficientemente elevado para que la reacción proceda a una velocidad adecuada; sin embargo, no se abandona el cálculo para facilitar la implementación en el simulador). Luego, para el sistema de inversión de flujo:

$$
G_{m, L, \min }=\frac{f_{L} S_{t o t} / 2}{t_{E L}}\left[\varepsilon_{L, I} \int_{0}^{L_{I}} \rho_{G}\left(T_{G}\left(t_{E C}, z\right)\right) d z+\varepsilon_{L, C} \int_{L_{I}}^{z^{*}} \rho_{G}\left(T_{G}\left(t_{E C}, z\right)\right) d z\right]
$$


donde $L_{I}$ es la longitud de la región inerte y $S_{\text {tot }}$ la sección transversal total del equipo.

Finalmente, para la implementación en el simulador, en ambos casos se modificó el valor mínimo del caudal de limpieza estableciendo un coeficiente de seguridad $\mathcal{C}=1.1$, que resultó adecuado para todas las condiciones estudiadas. Es decir:

$$
G_{m, L}=\mathcal{C} G_{m, L, \min }
$$

\subsection{Algoritmo interno}

Como se vio en el Capítulo 3, el modelo seleccionado para la simulación de los sistemas de inversión de flujo y para el regenerador, queda formado por un conjunto de ecuaciones diferenciales parciales y algebraicas.

Considerando que los algoritmos existentes para la integración de ecuaciones diferenciales ordinarias y algebraicas (DAEs, de sus siglas en inglés) se encuentran bien desarrollados, se optó por dividir la longitud total del equipo en un número $N$ de intervalos y aproximar las derivadas espaciales presentes en las ecuaciones de balances a través de un esquema de diferencias finitas atrasadas de orden " $n$ " ( $\left.\mathrm{DFA}_{n}\right)$. Con ello, la derivada de una variable " $x$ " cualquiera respecto de la posición axial $z$, evaluada para un tiempo $t$ y en la posición $z_{k}$, se puede representar mediante:

$$
\left(\frac{\partial x}{\partial z}\right)_{k}=\frac{1}{\Delta z} \sum_{q=0}^{n} b_{-q} x_{k-q}
$$

siendo $\left(\frac{\partial x}{\partial z}\right)_{k}=\frac{\partial x}{\partial z}\left(t, z_{k}\right), \Delta z$ el intervalo tomado $\left(\Delta z=\left(z_{k}-z_{k-n}\right) / n\right.$, constante en este caso), $b_{-q}$ los coeficientes de combinación y $x_{k-q}=x\left(t, z_{k-q}\right)$. Los coeficientes de combinación para una malla uniforme y distintos órdenes se dan en 
la Tabla 4.1. Los aspectos básicos de este tipo de métodos pueden encontrarse en Burden y Faires (2011).

La ventaja de este método es que permite reducir el sistema de ecuaciones diferenciales parciales a diferenciales ordinarias. Más aún, como en el modelo utilizado no se consideran en forma explícita términos de dispersión, la utilización de este método permite “avanzar" en la coordenada axial punto a punto, integrando en el tiempo las ecuaciones resultantes localmente, comenzando por el primer punto del sistema, donde la alimentación (variables de la fase gaseosa) está completamente definida. Esto resulta muy ventajoso, especialmente para los sistemas de inversión de flujo, en el cual el número de variables y la complejidad de las ecuaciones a resolver son muy elevados en la región catalíticamente activa (habrá 8 variables por punto: dos temperaturas y 6 fracciones molares).

Tabla 4.1: coeficientes de combinación para el método de DFA.

\begin{tabular}{ccccccc}
\hline Orden & $\boldsymbol{b}_{-5}$ & $\boldsymbol{b}_{-4}$ & $\boldsymbol{b}_{-3}$ & $\boldsymbol{b}_{-2}$ & $\boldsymbol{b}_{-1}$ & $\boldsymbol{b}_{0}$ \\
\hline $\mathbf{1}$ & --- & --- & -- & --- & -1 & 1 \\
\hline $\mathbf{2}$ & --- & --- & -- & $1 / 2$ & -2 & $3 / 2$ \\
\hline $\mathbf{3}$ & --- & --- & $-1 / 3$ & $3 / 2$ & -3 & $11 / 6$ \\
\hline $\mathbf{4}$ & --- & $1 / 4$ & $-4 / 3$ & 3 & -4 & $25 / 12$ \\
\hline $\mathbf{5}$ & $-12 / 60$ & $5 / 4$ & $-10 / 3$ & 5 & -5 & $137 / 60$ \\
\hline
\end{tabular}

No obstante, el método elegido requiere aún de consideraciones adicionales para poder aplicarlo en forma exitosa. De acuerdo con la expresión (4.14), para estimar la derivada espacial de una variable $x$ cualquiera en el punto $z_{k}$ y un tiempo $t$, se deberá conocer el valor de dicha variable para el mismo tiempo en las " $n$ " posiciones anteriores. Esto genera dos problemas desde el punto de vista de la implementación. El primero es que la información temporal de las variables en los puntos anteriores, luego de haber integrado las ecuaciones correspondientes, será discreta y no continua. La forma usual de sortear este inconveniente, y adoptada en los simuladores desarrollados, consiste en realizar una interpolación (cúbica) a partir de la información discreta. 
El segundo problema es cómo se resuelve la ausencia de información en el extremo menor del intervalo para los " $n$ - 1 " primeros puntos cuando el orden es mayor a 1 (se exceptúa al primer punto, debido a que las condiciones de alimentación o de continuidad en las variables de la fase gaseosa exime del uso de los balances en la misma para dicho punto). Existen dos formas básicas de sortear este inconveniente; una opción es sencillamente reducir el orden del esquema DFA en los primeros puntos, avanzando en la malla (y aumentando el orden simultáneamente) hasta alcanzar una posición donde se posea la información necesaria en su totalidad. La otra opción es similar a la anterior, pero incorpora puntos intermedios a fin de compensar la pérdida de precisión que implica bajar el orden. Esta última alternativa fue implementada en el simulador desarrollado. En la Figura 4.1 se muestra una representación esquemática de esta opción, supuesto se utilice un esquema de tercer orden.

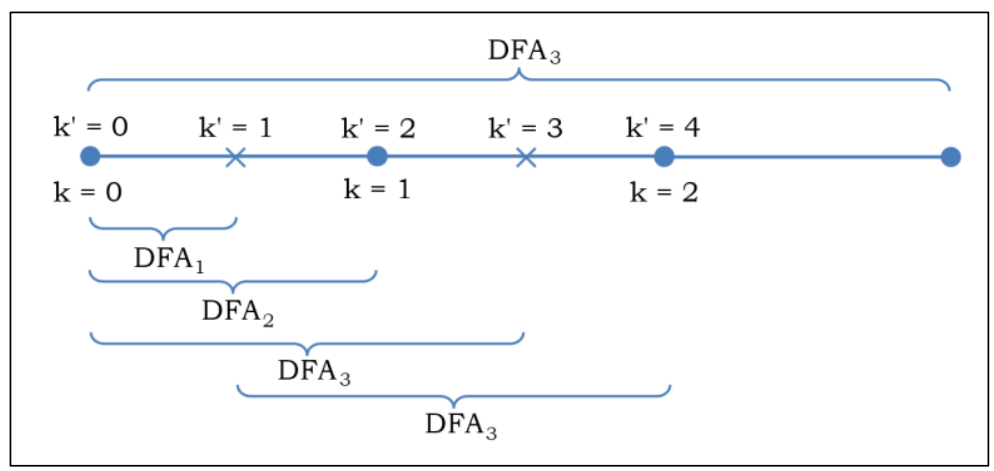

Figura 4.1: representación esquemática de la incorporación de puntos adicionales en los primeros puntos para un esquema $\mathrm{DFA}_{3}$.

El método aquí descrito se implementó en los simuladores desarrollados, utilizando una malla uniforme. En adición, para los sistemas de inversión de flujo se mantuvo una longitud de intervalo entre la región inerte y catalítica, en principio equivalente ${ }^{2}$, tratando el limite inferior de las fronteras inerte-catalizador y catalizador-inerte, de la misma manera que la descrita en el párrafo anterior. Finalmente, se trabajó en general con el esquema $\mathrm{DFA}_{2}$, pese a que en los simuladores desarrollados se puede seleccionar hasta un orden 5 .

\footnotetext{
$2 \mathrm{El}$ procedimiento en este caso fue calcular el número de intervalos en la región inerte y catalítica, exigiendo que los intervalos en cada región fuesen iguales y que la cantidad de intervalos totales resultantes se mantenga en el valor seleccionado. Luego, como difícilmente el número de intervalos en cada región calculados de esa manera serán números enteros, se redondean los valores obtenidos y se recalculan los intervalos de cada región.
} 
Esto se decidió en base a los resultados sobre la relación entre la precisión obtenida y el tiempo de cálculo requerido, como se analizará en la Sección 4.3.1.

Para la integración temporal de las ecuaciones diferenciales ordinarias resultantes, se utilizó la subrutina "ode15s" de MATLAB, la cual se basa en un método llamado genéricamente fórmulas de diferenciación numérica (NDF's de sus siglas en inglés) y que está recomendada para sistemas de DAEs de tipo "rígidos" (Shampine y col., 1999; Shampine y Reichelt, 1997).

Por último, se debe tener presente que en el caso del sistema regenerador-reactor, el reactor se considera en estado estacionario. Luego, las ecuaciones de balances correspondientes forman directamente un conjunto de DAEs (Ecs. 3.25 a 3.28). Como tal, las mismas también se resuelven con la subrutina "ode15s" de MATLAB para su simulación.

\subsubsection{Desempeño del algoritmo interno}

Considerando que los sistemas de inversión de flujo son, en cuanto a su resolución, más complejos que el sistema regenerador-reactor, el algoritmo utilizado para la aproximación de las derivadas espaciales solo se estudió en profundidad para el esquema de inversión de flujo convencional. Todos los parámetros relativos a la simulación se dan en la Tabla 4.2, donde $L_{e q}$ es la longitud total del equipo, $D_{e q}$ su diámetro, $f_{I}$ la proporción entre la longitud de las regiones inertes frente a la longitud total (abreviadamente "fracción de inerte"), $\Delta T_{a d}$ es el máximo salto térmico adiabático (se mantiene la proporción de acetato de etilo y etanol informada en el Capítulo 2), $N_{C}$ el número de celdas (canales) por unidad de superficie transversal y $\delta_{T}$ el espesor total de tabique.

Tabla 4.2: parámetros utilizados para el estudio de los algoritmos.

\begin{tabular}{ccccccc}
\hline $\begin{array}{c}\boldsymbol{L}_{\text {eq }} \\
{[\mathbf{m}]}\end{array}$ & $\begin{array}{c}\boldsymbol{D}_{\text {eq }} \\
{[\mathbf{m}]}\end{array}$ & $\begin{array}{c}\boldsymbol{f}_{\boldsymbol{I}} \\
{[\%]}\end{array}$ & $\begin{array}{c}\boldsymbol{t}_{\text {ciclo }} \\
{[\mathbf{s}]}\end{array}$ & $\begin{array}{c}\Delta \boldsymbol{T}_{\text {ad }} \\
{\left[{ }^{\circ} \mathbf{C}\right]}\end{array}$ & $\begin{array}{c}\boldsymbol{N}_{\mathbf{c}} \\
{\left[\mathbf{m}^{-2} \mathbf{]}\right.}\end{array}$ & $\begin{array}{c}\delta_{\boldsymbol{T}} \\
{[\mathbf{m}]}\end{array}$ \\
\hline 0.815 & 2.5 & 50 & 120 & 40 & $3.110^{5}$ & $3.17510^{-4}$ \\
\hline
\end{tabular}


Para llevar a cabo el estudio del algoritmo, se evaluó la predicción de la temperatura máxima (temporal y espacial) en el sólido en todo el lecho, $T_{S, \max }$, y de la emisión promedio de VOCs, $\left\langle y_{\text {VOCs }}\right\rangle$, Ecs. 3.20a y 3.20b, en el css (ver Sección 4.4 para la discusión sobre su determinación y las condiciones iniciales en el primer ciclo), utilizando diferentes números de intervalos totales, $N, \mathrm{y}$ órdenes del método DFA, registrando en cada caso el tiempo de cálculo requerido, $t_{c a l c}$. Las respuestas de estos tres parámetros con el número de intervalos considerados se presentan en la Figura 4.2.

En primer lugar, la aproximación de orden $1\left(\mathrm{DFA}_{1}\right)$, presenta una respuesta de tipo asintótica con convergencia muy lenta, tanto en $T_{S, \max }$ como en $\left\langle y_{\text {VOCS }}\right\rangle$ (Figuras 4.2a y 4.2b). Esto se puede explicar si se tiene presente que dicha aproximación será esencialmente equivalente a considerar que la fase gaseosa se comporta en cada intervalo como una serie de tanques en serie con mezcla perfecta en cada uno de ellos.

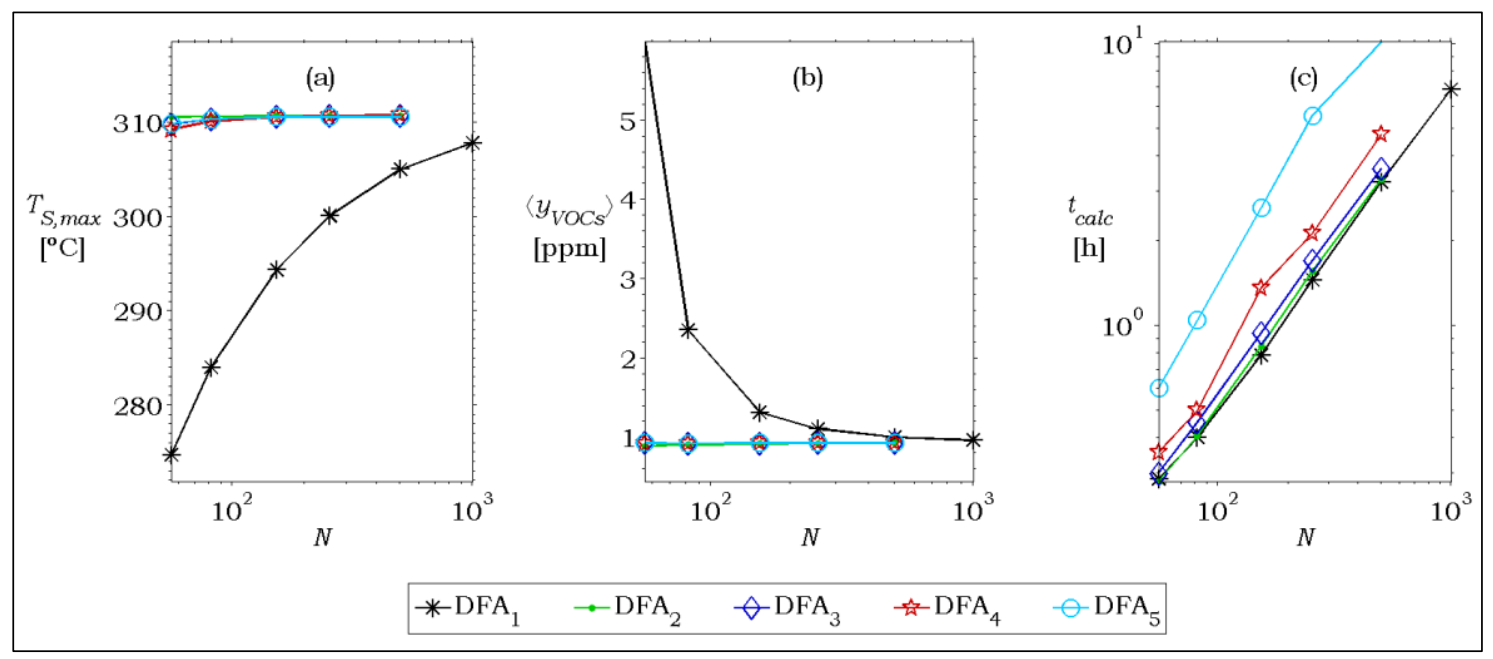

Figura 4.2: variables en el css y tiempo de cálculo requerido para diferentes órdenes y número de intervalos.

Por otro lado, el aumento en el orden de la aproximación permite disminuir rápidamente el requerimiento en la cantidad de intervalos, con un costo adicional en el tiempo de cálculo muy pequeño (Figura 4.2c), aunque como es habitual en los métodos numéricos de esta índole, el mayor beneficio se obtiene para bajos órdenes (i.e., el cambio es mucho más significativo al pasar del 
esquema $\mathrm{DFA}_{1}$ al $\mathrm{DFA}_{2}$ que en el resto). Esto último justifica la elección del esquema $\mathrm{DFA}_{2}$ para el simulador, como fuera anticipado.

A los fines de seleccionar un rango en la cantidad de intervalos a utilizar, se consideró como solución "exacta" a los datos obtenidos con el esquema DFA $\mathrm{D}_{5}$ 500 intervalos. Luego, llamando $T_{S, \max }^{\infty}$ e $\left\langle y_{V O C S}\right\rangle^{\infty}$ a la predicción en la temperatura máxima y la cantidad de VOCs emitida para las condiciones mencionadas, respectivamente, se definieron los errores relativos como:

$$
\begin{aligned}
& E_{T}=\frac{T_{S, \text { max }}-T_{S, \text { max }}^{\infty}}{T_{S, \text { max }}^{\infty}} \\
& E_{y}=\frac{\left\langle y_{V O C S}\right\rangle-\left\langle y_{\text {VOCS }}\right\rangle^{\infty}}{y_{V O C S}^{\text {ref }}}
\end{aligned}
$$

donde, $y_{V O C S}^{r e f}$ es el valor de referencia para la fracción molar total de VOCs en el efluente, equivalente a $1 \mathrm{ppm}$ (valor que, como se verá en el Capítulo 7, se adoptó como parte de los criterios de diseño). Cabe aclarar que para relativizar el error en la predicción de $\left\langle y_{\text {VOCS }}\right\rangle$, se consideró dicha referencia y no $\left\langle y_{V O C s}\right\rangle^{\infty}$, debido a que este último podría resultar muy pequeño y por lo tanto magnificar el valor de $E_{y}$ sin que esto fuese representativo.

Finalmente, con las definiciones (4.15) y (4.16), se graficaron las curvas paramétricas $t_{\text {calc }}(N)$ frente a $E_{T}(N)$ y $E_{y}(N)$, mostradas en las Figuras 4.3a y 4.3b. Considerando las Figuras 4.2 y 4.3 en conjunto, si se exige que $\max \left(\left|E_{T}\right| ;\left|E_{y}\right|\right) \leq 2 \%$, se puede concluir que será suficiente el uso de aproximadamente 100 intervalos para el esquema $\mathrm{DFA}_{2}$. No obstante, se debe reconocer que este resultado, si bien es representativo, puede depender de las condiciones operativas y configuración que se pretendan simular ${ }^{3}$. Previendo esto último, se utilizaron para todas las simulaciones 150 intervalos totales.

\footnotetext{
3 Por ejemplo, si el reactor tuviera una longitud mayor, sería esperable que la reacción proceda en una pequeña porción del mismo, pudiendo esto aumentar el error para una cantidad fija de intervalos.
} 


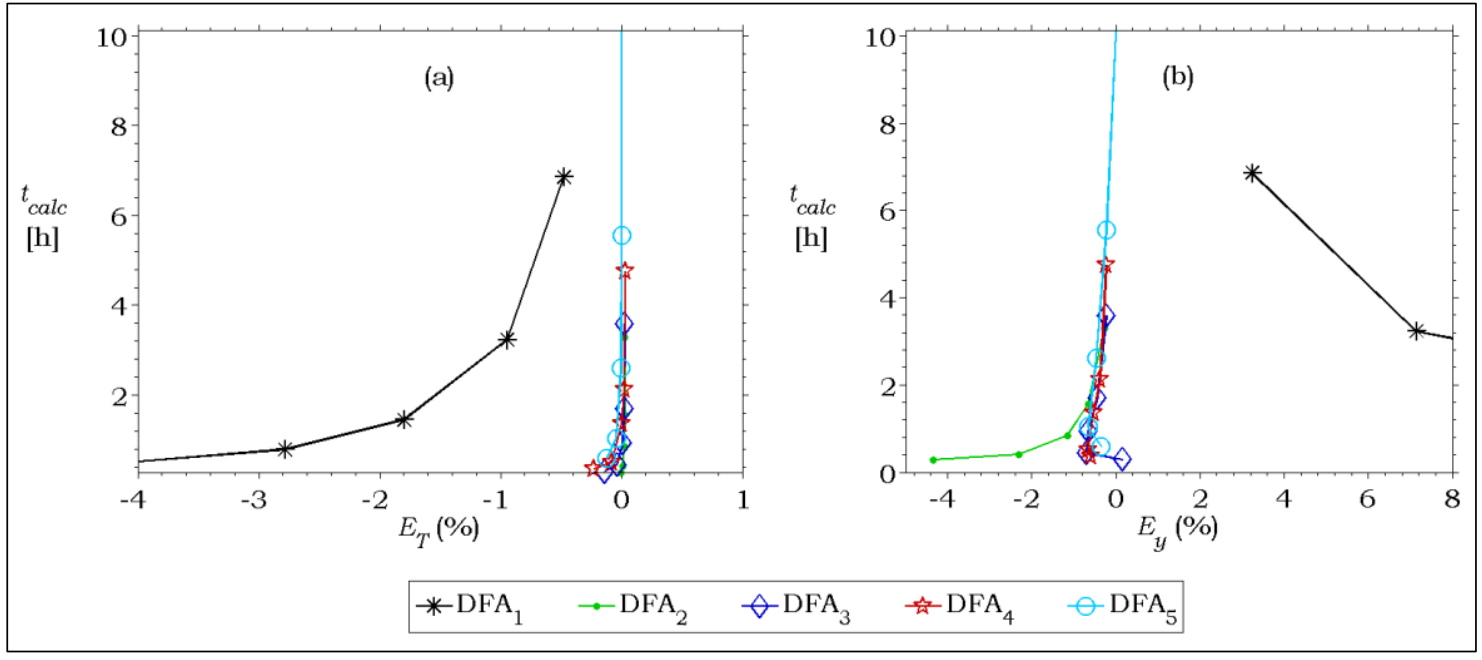

Figura 4.3: tiempo de cálculo requerido frente a los errores en las predicciones de las variables en el css para diferentes órdenes del esquema DFA.

\subsection{Algoritmo externo}

Previo a desarrollar la estrategia adoptada para la búsqueda del css, es menester aclarar que, si bien la misma no cambia de un sistema a otro, en el caso del sistema regenerador-reactor se debe definir un aspecto adicional. Como la simulación del regenerador depende de la cantidad convertida en el reactor $^{4}$, es necesario conocer dicha información. La opción adoptada en el presente estudio fue resolver el regenerador hasta la etapa de limpieza inclusive, con lo cual se tienen las condiciones de alimentación al reactor y se puede resolver este último. Luego, con las condiciones de salida del reactor, se puede completar el ciclo del regenerador. De esta manera, se incorpora la resolución del reactor en cada ciclo simulado del regenerador, aunque debe recordarse que esto no representa la evolución dinámica real del sistema, tal como fuera discutido en el Capítulo 3.

Habiendo aclarado esta diferencia y establecido un algoritmo que permite la resolución de las ecuaciones de balances en cada ciclo/semiciclo, podemos concentrarnos entonces en la estrategia para la búsqueda del css. La alternativa básica es lo que se podría definir como una búsqueda directa $\mathrm{y}$

\footnotetext{
4 Supuesta una temperatura de entrada al reactor, la cantidad de VOCs que hayan reaccionado determinarán las condiciones de salida del mismo y por ende, de entrada a la zona de calentamiento del regenerador.
} 
corresponde al uso, sin ninguna modificación, de las condiciones iniciales mencionadas en el Capítulo 3, partiendo desde una condición en la cual todo el lecho se encuentre a la temperatura $T_{S}^{i n i}\left(\mathrm{y}\right.$ siendo $\left.T_{G}^{i n i}=T_{S}^{i n i}\right)$ y el contenido de VOCs en la fase gaseosa sea uniforme e igual a un valor $y_{j, G}^{i n i}$.

Para definir dichos valores iniciales, debe tenerse presente que el nivel de temperatura en el lecho debe asegurar que la reacción pueda efectivamente desarrollarse durante los primeros ciclos, de forma tal que el sistema no evolucione hacia un estado apagado. Por esta razón y recordando que el catalizador considerado presenta una buena actividad a temperaturas por encima de $\operatorname{los} 200^{\circ} \mathrm{C}$, se estableció $T_{S}^{i n i}=300{ }^{\circ} \mathrm{C}$ para todos los casos. Por otro lado, si se considera el inicio de la búsqueda del css visualizando como podría llevarse a cabo el arranque de los equipos, pareciera ser lógico que los valores de $y_{j, G}^{\text {ini }}$ fuesen todos nulos. No obstante, en algunas situaciones esto perjudicaba la convergencia del sistema en el primer ciclo, por lo que se adoptó como condición para las fracciones molares $y_{j, G}^{i n i}=y_{j, G}^{0} / 1000$, permitiendo esto último eliminar dicho inconveniente.

Definidos los valores iniciales para la simulación del primer ciclo, se puede visualizar la búsqueda directa a través de la resolución de cada ciclo como un proceso iterativo sobre los perfiles iniciales del mismo. Esto es, suponiendo que se tiene un vector $\mathbf{X}$ (la variables vectoriales y matriciales se indicarán en "negrita" y "negrita y subrayadas", respectivamente) que define los perfiles iniciales (de todas las variables) para un dado ciclo y que se conoce la función $\mathbf{g}(\mathbf{X})$ que devuelve los perfiles finales al cabo de ese ciclo (la simulación del ciclo en cuestión), ambos con $N_{V}$ elementos ${ }^{5}$, la búsqueda del css quedaría definida por la búsqueda de la raíz de la función $\mathbf{G}(\mathbf{x})$, definida como

$$
\mathbf{G}(\mathbf{X})=\mathbf{g}(\mathbf{X})-\mathbf{X}
$$

\footnotetext{
$5 \mathrm{El}$ número de elementos del vector $\mathbf{X}$ dependerá de la cantidad de intervalos considerados y, en el caso de los sistemas de inversión de flujo, de la proporción de inerte. Así por ejemplo, para cualquiera de los esquemas de inversión de flujo, suponiendo que se tomaron $N$ intervalos y que todo el lecho es catalíticamente activo, el número de elementos $N_{V}$ será igual a $8 N+4$.
} 
Luego, la búsqueda directa se puede escribir como $\mathbf{X}^{(p+1)}=\mathbf{g}\left(\mathbf{x}^{(p)}\right)$, donde “ $p$ ” indica el número de iteración en cuestión (equivalente a un ciclo para este caso). Un método de este tipo corresponde a los llamados "métodos sin memoria", pues solo trabajan con la información de la iteración inmediata anterior para determinar el nuevo vector $\mathbf{X}^{(p+1)}$. Resulta natural entonces preguntarse si es posible acelerar el proceso de búsqueda reteniendo información de iteraciones anteriores (por ejemplo, en forma similar al uso del método de Aitken en el algoritmo de punto fijo para una única variable, conocido como algoritmo de Steffesen -Burden y Faires, 2011-). Una alternativa para esto viene dada por la generalización del método llamado "mezclado o combinación de Anderson" descrito por Eyert (1996) y llamada por el autor como "Extended Anderson Mixing", que, como se demuestra allí, resulta equivalente a la forma generalizada del segundo método de Broyden.

El algoritmo de Anderson parte de una forma un poco más general para la búsqueda directa, que se puede escribir como:

$$
\mathbf{X}^{(p+1)}=\mathbf{X}^{(p)}+\sigma^{(p)} \mathbf{G}\left(\mathbf{X}^{(p)}\right)
$$

donde $\sigma^{(p)}$ es un "parámetro de mezcla" asociado a la iteración $p$-ésima. Lógicamente, si se toma $\sigma^{(p)}=1$ se obtiene el esquema de la búsqueda directa mencionado previamente.

Definida la expresión (4.18), la propuesta del algoritmo es modificar la misma a partir de una combinación lineal de la información procedente de las " $M$ " iteraciones anteriores mediante:

$$
\mathbf{X}^{(p+1)}=\mathbf{X}^{(p)}+\sigma^{(p)} \mathbf{G}\left(\mathbf{X}^{(p)}\right)-\left[\underline{\underline{\Delta}}+\sigma^{(p)} \underline{\Delta \mathbf{G}}\right] \mathbf{\gamma}^{(p)}
$$

en donde cada columna " $m$ " de las matrices $\underline{\boldsymbol{\Delta x}}$ y $\underline{\boldsymbol{\Delta G}}$ de $N_{V} \mathrm{x} M$ están definidas como: 


$$
\begin{array}{ll}
\Delta \mathbf{X}_{m}=\mathbf{X}^{(p-M+m)}-\mathbf{X}^{(p-M+m-1)} & m=1 . . M \\
\Delta \mathbf{G}_{m}=\mathbf{G}\left(\mathbf{X}^{(p-M+m)}\right)-\mathbf{G}\left(\mathbf{X}^{(p-M+m-1)}\right) & m=1 . . M
\end{array}
$$

y el vector $\boldsymbol{\gamma}^{(p)}$ se obtiene de la resolución del sistema de ecuaciones lineales de $M \times M$ dado por:

$$
\underline{\mathbf{A}} \boldsymbol{\gamma}^{(p)}=\left[\underline{\Delta \mathbf{G}^{T}} \mathbf{G}\left(\mathbf{X}^{(p)}\right)\right]
$$

Teniendo en cuenta que $\delta_{h m}$ representa la función delta de Kronecker, cada elemento de la matriz $\underline{\mathbf{A}}$ se puede escribir como:

$$
A_{h m}=\left(1+\delta_{h m} w_{0}^{2}\right)\left[\mathbf{\Delta} \mathbf{G}_{h}^{T} \boldsymbol{\Delta G}_{m}\right]
$$

en cuya definición se incluye el factor $w_{0}^{2}$ para prevenir el caso en que el determinante de la matriz $\underline{\Delta \mathbf{G}} \underline{\Delta \mathbf{G}}^{T}$ fuese cero.

Las Ecs. (4.19) a (4.23) definen el procedimiento para obtener el nuevo vector de condiciones iniciales para simular un nuevo ciclo, supuesto se hubieran definido los valores de $\sigma^{(p)}, w_{0}^{2}$ y la cantidad de iteraciones previas, $M$, a ser tenidas en cuenta. Eyert (1996), sugiere el uso de $\sigma^{(p)}=\sigma=1 / 2$ (independiente de la iteración), $w_{0}^{2}=10^{-4}$ y $M=4$ o $M=5$. A pesar del valor sugerido para $\sigma^{(p)}$, dicho parámetro se adoptó como 1 , debido a que esto es lo que surge de forma natural en el sistema (verificándose que para este caso resulta más eficiente), mientras que se mantuvo el valor de $w_{0}^{2}$ sugerido por el autor. En cuanto al parámetro $M$, se estudió su impacto en el número de iteraciones y tiempo de cálculo requeridos, verificándose que los valores sugeridos representan la situación más conveniente, como se verá en la Sección 4.4.2. 


\subsubsection{Criterios de corte}

Otro aspecto importante en la implementación de los modelos presentados en el Capítulo 3 y de los algoritmos descritos en el presente, son los criterios de corte a utilizar, es decir, ¿cuándo puede concluirse que el estado del sistema corresponde efectivamente al css? Lógicamente, como sucede con todo proceso que alcanza un estado dado en forma asintótica, se debe definir una tolerancia para poder responder a la pregunta planteada. Es decir, el css se determina a menos de esa tolerancia.

Recordando que la temperatura del sólido domina el comportamiento del sistema, se estableció el control con dos condiciones simultáneas entre iteraciones sucesivas: la variación en los perfiles de la temperatura del sólido y la variación sobre la predicción de la cantidad de VOCs emitidos. Luego, definiendo la tolerancia relativa como "rtol" (igual para todas las variables), las absolutas como " $a t o l_{T}$ " (para la temperatura) y "atol VOCs) y llamando a los componentes en el vector $\mathbf{X}$ asociados a la temperatura de la fase sólida como $\mathbf{x}_{T}$, dicho control se estableció como:

$$
\begin{aligned}
& \left\|\mathbf{X}_{T}^{(p+1)}-\mathbf{X}_{T}^{(p)}\right\|_{2} \leq \max \left\{(N+1)^{1 / 2} \text { atol }_{T} ; \operatorname{rtol}\left\|\mathbf{X}_{T}^{(p)}\right\|_{2}\right\} \\
& \left|\left\langle y_{\text {VOCS }}\right\rangle^{(p+1)}-\left\langle y_{\text {VOCS }}\right\rangle^{(p)}\right| \leq \max \left\{\text { atol }_{y} ; \operatorname{rtol} \sum_{j=1}^{3} y_{j, G}^{0}\right\}
\end{aligned}
$$

en donde \|\|$_{2}$ representa la norma euclidea o norma 2. Los valores adoptados para $r t o l$, atol $l_{T}$ y atol fueron $310^{-6}, 210^{-3} \mathrm{~K}$ y $510^{-9}$, respectivamente. Cabe aclarar que con el valor de $a_{t o l}$ indicado, la restricción (4.24b) queda en realidad controlada enteramente por el mismo. Esta situación se mantuvo debido a que valores menores carecen de sentido práctico y podrian generar un conflicto con los valores adoptados para el resolvedor "ode $15 \mathrm{~s}$ " con el que se resuelven las ecuaciones diferenciales ordinarias en cada punto. 


\subsubsection{Desempeño del algoritmo externo}

Para analizar el desempeño del algoritmo utilizado para la búsqueda del css, se optó nuevamente por el esquema de inversión de flujo convencional, debido a su mayor complejidad, como fuera ya señalado. En todos los casos, los parámetros utilizados en la simulación son los dados en la Tabla 4.2.

En la Figura 4.4 se muestran los resultados en la predicción de la temperatura máxima en el lecho y de la cantidad de VOCs emitida, en función del número de iteraciones necesarias para satisfacer las condiciones (4.24), en donde se ha considerado que $M=0$ corresponde a la búsqueda directa.

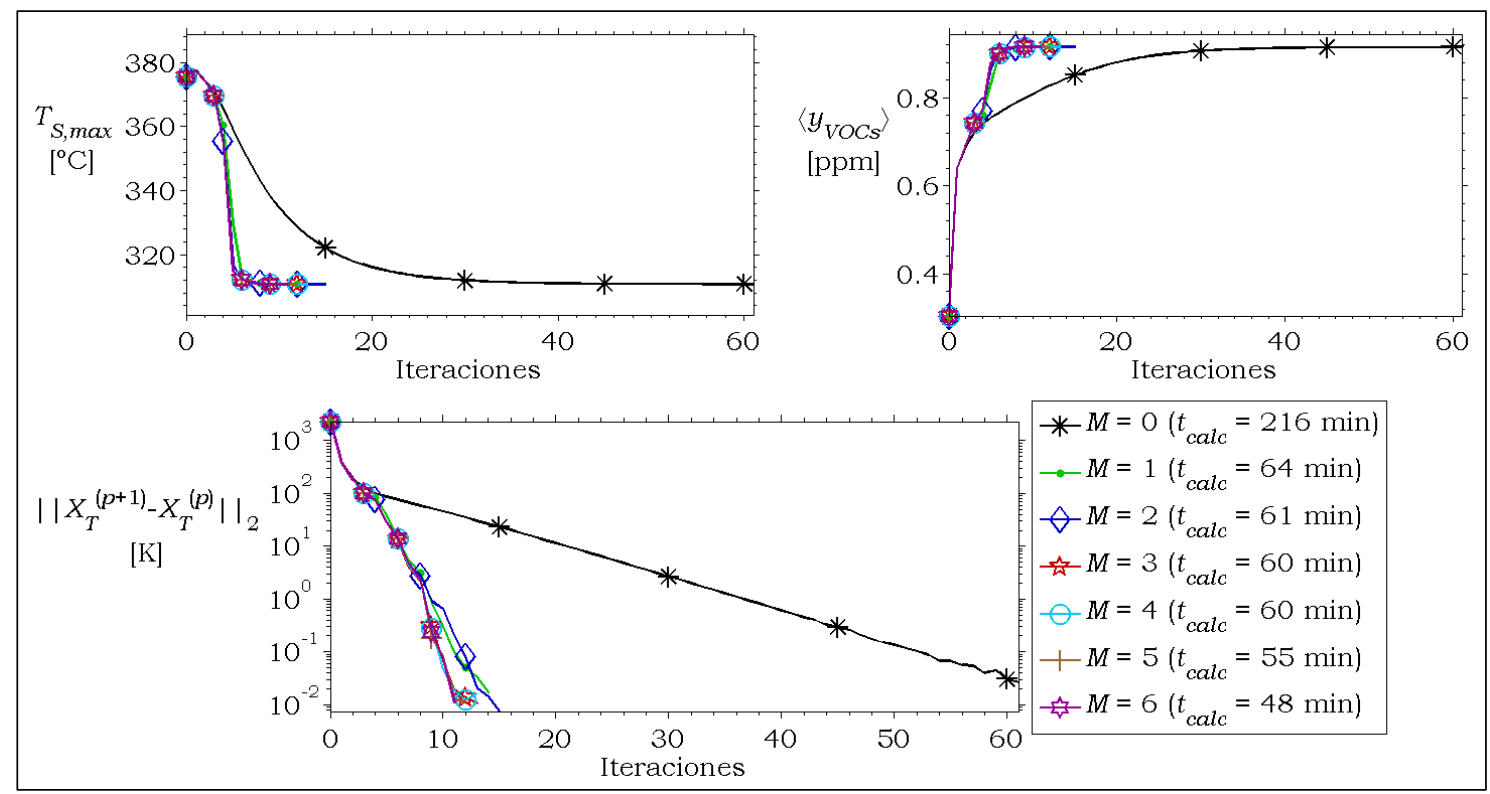

Figura 4.4: respuesta durante la predicción del css para distintos valores del parámetro $M$.

Se puede observar que la convergencia al css resulta muy lenta para $M=0$, principalmente debido al incumplimiento de (4.24a). Si bien esto podría inducir a pensar que dicha condición es muy restrictiva, se han encontrado casos en los cuales el css del sistema corresponde a un estado "apagado", pero como consecuencia de la cercanía al límite entre el estado apagado y el encendido, la temperatura máxima en el lecho varía muy lentamente durante un número elevado de iteraciones (ciclos en este caso), hasta un ciclo en que el nivel térmico del lecho comienza a disminuir en forma más drástica.

Por otro lado, la retención de al menos un dato adicional en las iteraciones efectuadas (es decir, $M \geq 1$ ) resulta claramente beneficioso. De hecho, para 
$M=5$ se llega a disminuir el número de iteraciones requeridas en 5 veces (de 61 a 12) y el tiempo de cálculo en 4 veces. En base a los resultados obtenidos y siguiendo las sugerencias dadas por Eyert (1996), se adoptó para todas las simulaciones y para los tres esquemas a estudiar, $M=5$.

Por último, cabe resaltar que la "función" de residuos $\mathbf{G}(\mathbf{X})$ con la que se computan los nuevos valores para los perfiles iniciales en cada iteración corresponde directamente a la diferencia entre los perfiles iniciales y finales en la iteración, por lo cual es esperable que si el css correspondiente resultase apagado, se produzca una gran variación en los perfiles en alguna iteración y, por ende, los residuos aumenten. En esa circunstancia, el intento de acelerar la convergencia a través del algoritmo descrito en esta sección podría llevar a comportamientos oscilatorios $\mathrm{y}$, contrario al objetivo propuesto, ralentizar la convergencia. Por esta razón, previo a la estimación de los nuevos perfiles iniciales, es conveniente verificar que la norma de los $M$ vectores almacenados

de $\mathbf{G}(\mathbf{X})$ sean decrecientes y solo aplicar la aceleración en ese caso. Si no se verifica lo anterior, la aceleración se omite y se procede con la búsqueda directa (expresión 4.18), al menos hasta disponer de $M$ valores consecutivos de $\mathbf{G}(\mathbf{X})$ cuyas normas sean decrecientes.

\subsection{Algoritmo para el reactor en contra-corriente}

En el Capítulo 3 se describió el reactor en contra-corriente (RCC) como modelo correspondiente a la aproximación para altas frecuencias de ciclo, a través de las Ecs. (3.32) a (3.38). Dicho conjunto representa un sistema de ecuaciones diferenciales y algebraicas ordinarias, pero de "condiciones de borde" en lugar de iniciales.

Las alternativas básicas para resolver este tipo de problemas son dos. Por un lado, se pueden suponer los valores no conocidos en un extremo, utilizar un resolvedor convencional ("ode15s" por ejemplo) y al finalizar la resolución verificar la igualdad en el otro extremo (método de shooting). La segunda alternativa consiste en discretizar la variable axial $z$ para aproximar simultáneamente las derivadas en un conjunto de nodos, lo cual permite reducir el sistema de DAEs a solo un sistema de ecuaciones algebraicas. 
De estos métodos, el primero fue probado de dos maneras. En la primera, se suponía un valor para la temperatura y fracciones molares en la fase gaseosa a la salida y se verificaba la igualdad de estas variables en la posición central del canal, con lo cual se corregían los valores propuestos. En la segunda, se suponian los valores de esas variables en el centro del canal y se verificaban los valores a la entrada. Ninguna de estas opciones fue adecuada, mostrando grandes problemas de estabilidad, siendo muy difícil la convergencia, a menos que se partiera de valores supuestos muy cercanos a la solución.

Como consecuencia de lo anterior, el método de shooting fue desechado y se trabajó aproximando las derivadas mediante un esquema diferencias finitas centradas (un punto hacia cada lado). En todos los casos, se tomaron 100 intervalos, considerando los resultados alcanzados para el sistema de inversión de flujo presentado en la Sección 4.3.1.

De esta manera, se obtiene un sistema de ecuaciones algebraicas, que puede ser resuelto con un resolvedor convencional que permita manejar un sistema multidimensional. En este caso, se trabajó con la función "fsolve" de MATLAB, que utiliza el algoritmo de la "región de confianza" (o trust regiondogleg). Este esquema resultó ser muy eficiente y prácticamente no se encontraron problemas de convergencia. Adicionalmente, a diferencia de los sistemas de inversión de flujo que requieren un tiempo de cálculo elevado (normalmente mayor a 30 minutos), para este modelo se requiere apenas unos cuantos segundos.

\subsection{Conclusiones y comentarios finales}

En este capítulo se presentaron los algoritmos utilizados para la simulación de cada sistema, tanto para la resolución de un ciclo/iteración dado (algoritmo interno) como para la búsqueda del css (algoritmo externo).

Se corroboró que el esquema de diferencias finitas atrasadas (DFA), particularmente el de orden 2, resulta muy eficiente para la simulación del sistema de inversión de flujo. En el caso del esquema de orden 1, se comentó su similitud con la aproximación al comportamiento de la fase gaseosa en cada intervalo como una serie de tanques agitados con mezcla perfecta. Como se verificó a través del estudio realizado, esto genera que la cantidad de intervalos necesa- 
rios para que la solución se pueda considerar independiente del número de intervalos resulte muy elevado (>1000). Por otro lado, esquemas de orden mayor a 2 no aportan ventajas significativas respecto del $\mathrm{DFA}_{2}$. Por estas razones, en todos los simuladores desarrollados se utilizó este último.

En cuanto a la determinación del estado estacionario cíclico, css, se implementó un método para acelerar la convergencia durante su determinación, pudiendo corroborar que el mismo es sumamente eficiente para valores del parámetro $M$ de 4 o 5 , tal como recomienda Eyert (1996). Esto permitió una reducción en el tiempo de cálculo requerido a $1 / 4$ del valor correspondiente a la simulación sin tener en cuenta iteraciones previas.

Para finalizar, se dieron los lineamientos principales para la simulación del RCC, basada enteramente en el método presentado para el sistema de inversión de flujo, indicando los problemas que surgen si se pretende utilizar un esquema tipo shooting. El método finalmente utilizado, aproximando las derivadas mediante un esquema diferencias finitas centradas, resultó sumamente eficiente, alcanzándose la solución en unos pocos segundos. 


\section{Capítulo 05}

\section{- Análisis de los sistemas de inversión de flujo}

- Consideraciones generales. Análisis preliminar mediante el reactor en contra-corriente

- Tiempo de ciclo

- Longitud del equipo

- Contenido total de VOCs

- Caída en la actividad del catalizador

- Aspectos adicionales 

En este capítulo se presenta un análisis de la respuesta de los sistemas de inversión de flujo frente a variaciones en los parámetros operativos y de diseño más relevantes, entendiendo que para lograr un diseño satisfactorio del mismo resulta imprescindible conocer previamente su comportamiento.

Particularmente, se consideraron las siguientes variables:

* tiempo de duración del ciclo,

* volumen total de equipo para una sección transversal dada (traducido en la longitud total),

* proporción de la región inerte,

* contenido total de VOCs en la corriente a tratar (traducida en el máximo salto térmico de la misma),

* caída en la actividad del catalizador.

Se excluye, por ejemplo, la velocidad másica, la cual se fijó para obtener una adecuada pérdida de carga, $\lesssim 0.1$ bar para todos los casos, aspecto que será abordado con mayor detalle en el Capítulo 7. Para un caudal másico total dado, esto define inmediatamente el diámetro del equipo.

La condición base para realizar el análisis se muestra en la Tabla 5.1, donde $L_{e q}$ es la longitud total del equipo, $D_{e q}$ su diámetro, $f_{I}$ la proporción entre la longitud de las regiones inertes frente a la longitud total (abreviadamente fracción de inerte), $t_{\text {ciclo }}$ el tiempo de duración del ciclo, $\Delta T_{a d}$ el máximo salto térmico adiabático (se mantiene la proporción de acetato de etilo y etanol informada en el Capítulo 2), $N_{C}$ el número de celdas (canales) por unidad de superficie transversal y $\delta_{T}$ el espesor total de tabique. La fracción de inerte, $f_{I}$, se ha elegido como un parámetro para todos los casos, considerando que la misma está distribuida en partes iguales en los extremos del equipo. Como se verá a lo largo de este capítulo, esta elección se debe a que la fracción de inerte en el lecho condiciona en gran medida el tipo de comportamiento observado al modificar el resto de las variables.

Cabe aclarar que el análisis se hace casi exclusivamente para el esquema de inversión de flujo convencional. No obstante, se presentan algunos resulta- 
dos para el esquema rotativo frente a la variación del tiempo de ciclo ${ }^{6}$. Como se verá en la Sección 5.1, la similitud de los resultados obtenidos permite conducir el resto del análisis considerando únicamente el esquema convencional.

Tabla 5.1: parámetros utilizados para el estudio de los sistemas de inversión de flujo.

\begin{tabular}{ccccccc}
\hline $\begin{array}{c}\boldsymbol{L}_{\text {eq }} \\
{[\mathbf{m}]}\end{array}$ & $\begin{array}{c}\boldsymbol{D}_{\text {eq }} \\
{[\mathbf{m}]}\end{array}$ & $\begin{array}{c}\boldsymbol{f}_{I} \\
{[\%]}\end{array}$ & $\begin{array}{c}\boldsymbol{t}_{\text {ciclo }} \\
{[\mathbf{s}]}\end{array}$ & $\begin{array}{c}\Delta \boldsymbol{T}_{\boldsymbol{a d}} \\
{\left[{ }^{\circ} \mathbf{C}\right]}\end{array}$ & $\begin{array}{c}\boldsymbol{N}_{\mathbf{c}} \\
{\left[\mathbf{m}^{-2}\right]}\end{array}$ & $\begin{array}{c}\delta_{T} \\
{[\mathbf{m}]}\end{array}$ \\
\hline 0.815 & 2.5 & {$[0 ; 25 ; 50 ; 75]$} & 120 & 40 & $3.110^{5}$ & $3.17510^{-4}$ \\
\hline
\end{tabular}

En todos los casos, la respuesta del sistema se da en términos de la temperatura máxima del sólido en todo el lecho (máximo en posición y tiempo), $T_{S, \max }$, y de la emisión total de VOCs promedio en el ciclo, $\left\langle y_{V O C s}\right\rangle$, entendiendo que estas son las dos variables más relevantes en el sistema.

Cabe recordar que en el presente estudio no se hará, en términos generales, referencia a la conversión promedio de VOCs, pese a ser algo frecuente en la bibliografía específica. En su lugar, se retendrá el concepto de emisión total de VOCs promediada en el ciclo, que es la condición establecida como criterio de "límite de descarga", resultando por lo tanto más adecuado. Para ejemplificar la relación entre ambas magnitudes, se puede señalar que el limite establecido en el Capítulo 2 (9 ppm) correspondería a una conversión de VOCs cercana al 99\% para el caso base considerado en este capítulo.

\subsection{Consideraciones generales. Análisis preliminar me- diante el reactor en contra-corriente}

El reactor en contra-corriente $(\mathrm{RCC})$ presentado en el Capítulo 3, permite predecir la forma de los perfiles de temperatura y fracción molar para la operación del sistema de inversión de flujo a muy bajos tiempos de ciclo,

\footnotetext{
${ }^{6} \mathrm{El}$ análisis del impacto que posee la fracción de la sección total destinada a la limpieza, $f_{L}$, para el esquema de inversión de flujo rotativo, se reserva para el Capítulo 7 donde se aborda el diseño de los esquemas de inversión de flujo. Este se debe a que es esperable una mayor influencia de $f_{L}$ sobre la pérdida de carga total (la alimentación a la región de limpieza corresponde a la salida de la etapa de reacción -ver Figura 3.1-) y, por lo tanto, es recomendable incluir este factor en el análisis.
} 
incorporando todas las particularidades del intercambio de calor regenerativo con simultánea reacción química. Por esta razón, se comenzará discutiendo el comportamiento de este sistema para los parámetros dados en la Tabla 5.1 (excepto el tiempo de ciclo, que carece de significado en este modelo).

En la Figura 5.1 se condensan los perfiles de temperatura en ambas fases y de fracción molar en la fase gaseosa, para cada uno de los valores de $f_{I}$. Cabe aclarar que se han graficado únicamente los perfiles de la región superior, de acuerdo con la Figura 3.4, para facilitar luego su comparación con los perfiles en el sistema de inversión.

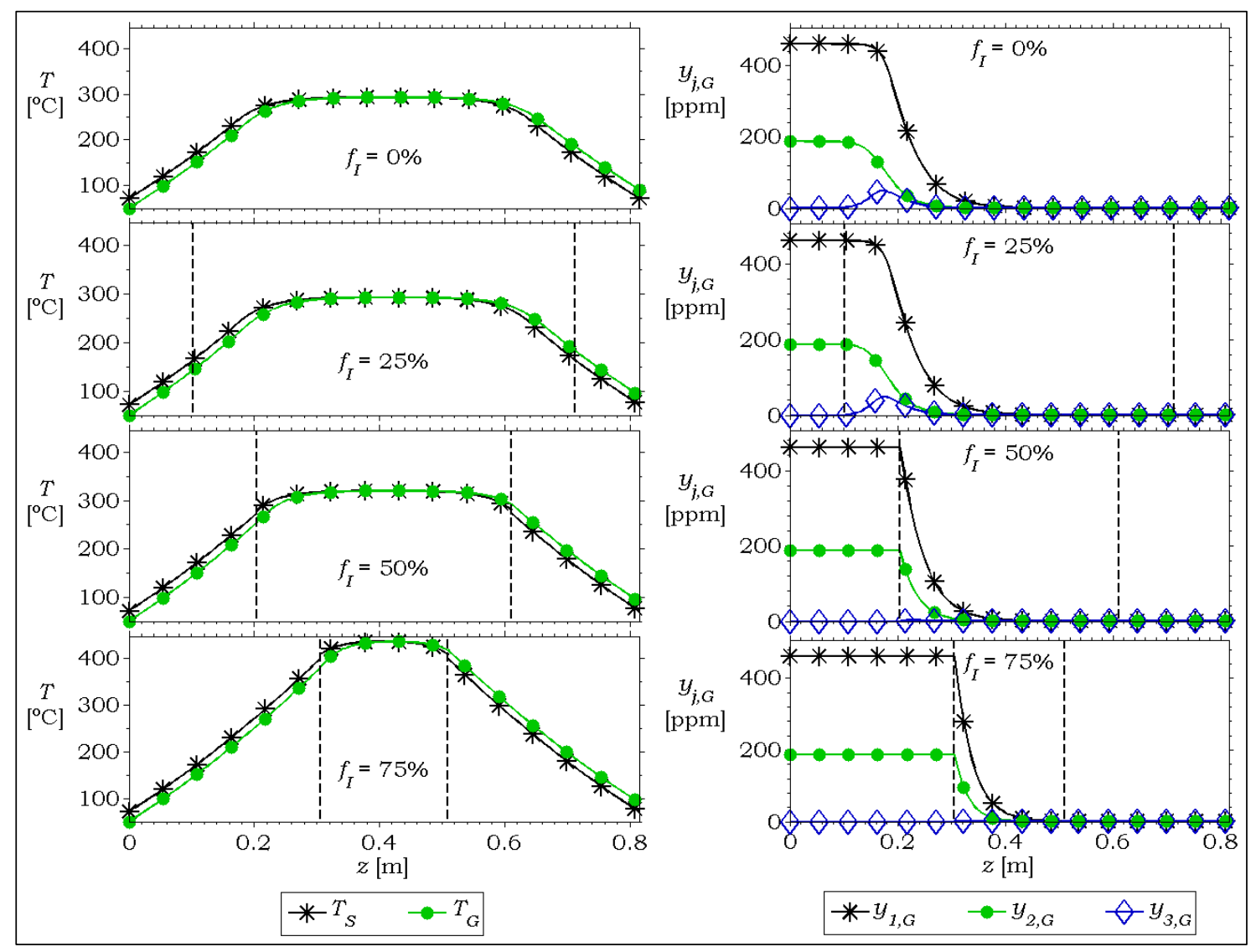

Figura 5.1: efecto de la fracción de inerte en el lecho según el RCC. Las líneas de trazos verticales indican los límites inerte-catalizador. (Los símbolos en cada curva se agregaron para facilitar la lectura, sin indicar necesariamente todos los puntos calculados. Este criterio será utilizado en diversas figuras en los capitulos posteriores).

En primer lugar, se puede observar que para $f_{I}=0$ existe una porción del lecho que es utilizada exclusivamente para el intercambio de energía, debido a que la temperatura resulta significativamente inferior a la de ignición, $T_{i g n}$ 
(siendo esta última cercana a los $200^{\circ} \mathrm{C}$, para todos los componentes). Esta región, en donde la corriente gaseosa eleva su temperatura sin reaccionar, la identificaremos como "región de precalentamiento". Luego, resulta inmediato que si el material catalítico de dicha región se reemplaza por material inerte, con las mismas o muy similares características, no se debería observar ningún efecto significativo en el comportamiento del sistema. Esto se ve reflejado en los perfiles de temperatura y fracción molar en la configuración con $f_{I}=25 \%$, donde no hay cambios apreciables respecto del caso con $f_{I}=0$. De hecho, en todos los casos analizados en las secciones siguientes, las curvas de temperatura máxima y emisión de VOCs resultaron prácticamente idénticas (quedan superpuestas entre sí); por esta razón, solo se mostrará en los análisis de cada caso la curva para $f_{I}=25 \%$.

En segundo lugar, para los casos con $f_{I}=0$ y $f_{I}=25 \%$, se observa la formación de una amplia "meseta" en los perfiles de temperatura en la región central del reactor. Adicionalmente, se puede ver que la formación y acumulación de acetaldehído en estos casos es apreciable, aspecto no menor si se considera que el mismo participa del término de inhibición en las expresiones cinéticas. Esta acumulación se debe a que el etanol posee una menor temperatura de ignición que el acetaldehído, por lo que el primero comienza a reaccionar cuando el nivel térmico del lecho es aún bajo para que el acetaldehído se consuma. Esta situación no se verifica cuando la temperatura en la región catalitica es más elevada como consecuencia de la mayor proporción de región inerte.

La formación de la meseta en la región central se puede explicar si se considera la representación del RCC mostrada en la Figura 3.4. La misma se repite aquí en la Figura 5.2, pero se han adicionado dos lineas verticales, indicando una posición hipotética para la cual ya se podría haber liberado toda la energía. Si se recuerda que los perfiles de temperatura en uno y otro lado deben ser simétricos, resulta inmediato que en la región comprendida entre dichas líneas no habrá un aumento de la temperatura, permaneciendo la misma aproximadamente constante (esto es similar, por ejemplo, a lo que ocurre hacia la salida de un reactor adiabático convencional, si el tiempo de residencia es muy elevado). 


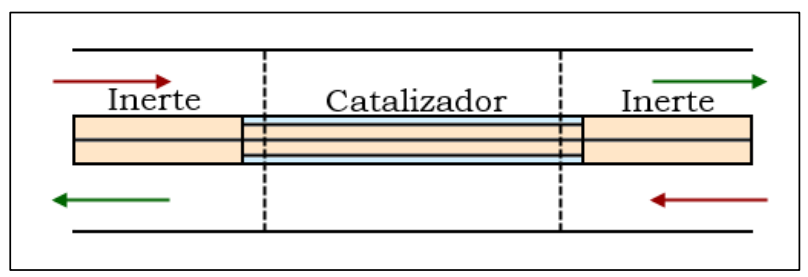

Figura 5.2: reactor hipotético con el gas en contracorriente.

En el caso extremo, puede suponerse que una vez superada la temperatura de ignición de los VOCs, $T_{i g n}$, la reacción es completa y procede en una zona muy estrecha (Eigenberger y col., 2007), situación en la cual se puede considerar:

$$
T_{\mathrm{S}, \max } \approx T_{i g n}+\Delta T_{a d}
$$

donde $T_{S, \max }$ representa la temperatura máxima en el lecho y $\Delta T_{a d}$ el máximo salto térmico adiabático, dado en este caso por:

$$
\Delta T_{a d}=\frac{1}{M_{\text {Aire }} c_{p G}^{0}}\left[\left(-\Delta H_{1}^{\#}\right) y_{1, G}^{0}+\left(-\Delta H_{2}^{\#}\right) y_{2, G}^{0}\right]
$$

siendo, $M_{\text {aire }}$ el peso molecular del aire, $c_{p G}^{0}$ y $\Delta H_{j}^{\#}$ el calor específico del aire y las entalpías de combustión del acetato de etilo y del etanol, evaluados a la temperatura de alimentación, e $y_{j, G}^{0}$ las fracciones molares del acetato de etilo y del etanol en la alimentación.

Por otro lado, cuando la cantidad de material inerte es significativa (para este caso, $f_{I} \gtrsim 50 \%$ ), el sistema dispondrá de una zona de precalentamiento mayor, comparada con el caso descrito en los párrafos anteriores. Esto se debe a que, aun cuando la temperatura de la fase gaseosa hubiera superado la temperatura de ignición, la reacción no tendrá lugar hasta que los reactivos alcancen la región catalítica. Como consecuencia, si el estado estacionario del sistema resulta encendido, la energía recuperada y lógicamente el nivel térmi- 
co en el lecho, serán mayores, lo que permitirá compensar con creces la disminución de la longitud catalítica. No obstante, si la fracción de material inerte se aumentara aún más, se podría llegar a una situación en la cual la menor longitud de la región catalítica ya no pueda ser compensada. Adicionalmente, como consecuencia del mayor nivel térmico en el reactor y la elevada energía de activación de la reacción de combustión del acetaldehído, el mismo no alcanza a formarse y acumularse en forma significativa en este caso, como se puede observar en los perfiles de fracciones molares para $f_{I}=50 \%$ y $f_{I}=75 \%$.

En este caso, en la región inerte la variación de la temperatura con la posición para ambas fases depende de la fuerza impulsora para el intercambio de energía. A partir de las Ecs. (3.32) a (3.34), considerando propiedades constantes, eliminando los términos correspondientes a la reacción química y por un balance de energía global en el sistema, se puede concluir que $\overleftarrow{T_{G}}-\vec{T}_{G}=\Delta T_{a d}$ y que $T_{S}=\left(\vec{T}_{G}+\overleftarrow{T_{G}}\right) / 2$, para toda la posición axial. Con ello, sumando las Ecs. (3.33) y (3.34) se obtiene:

$$
\frac{d \vec{T}_{G}}{d z}=\frac{a_{v} h_{m o d}}{G c_{p G}} \frac{\Delta T_{a d}}{2}
$$

Expresión que resulta completamente análoga a la propuesta por Nieken y col. (1995). Luego, integrando la expresión (5.3) entre $z=0$ y $z=L_{I}=L_{e q} f_{I} / 2$, la temperatura del gas al final de dicha región quedará definida por:

$$
\vec{T}_{G}\left(L_{I}\right)=T_{G}^{0}+\frac{a_{v} h_{m o d} \Delta T_{a d} f_{I}}{4 G c_{p G}} L_{e q}
$$

Manteniendo la suposición realizada por Eigenberger y col. (2007), si $\vec{T}_{G}\left(L_{I}\right)$ resulta menor a la temperatura de ignición, parte del material catalítico será utilizado como zona de precalentamiento hasta alcanzar dicho valor. Por el 
contrario, si $\vec{T}_{G}\left(L_{I}\right)$ es mayor a la temperatura de ignición, toda la energía será liberada al alcanzar la región catalítica, pero a una temperatura mayor. Considerando lo anterior, se puede modificar la expresión (5.1) tomando el máximo valor entre $T_{\text {ign }}$ y $\vec{T}_{G}\left(L_{I}\right)$, según la Ec. (5.4):

$$
T_{G, \text { max }}^{\rightarrow} \approx T_{S, \text { max }} \approx \max \left\{T_{i g n} ; T_{G}^{0}+\frac{a_{v} h_{m o d} \Delta T_{a d} f_{I} L_{e q}}{4 G c_{p G}}\right\}+\Delta T_{a d}
$$

Como se verá en las siguientes secciones, esta aproximación junto con los razonamientos en términos de la región de precalentamiento, permiten explicar cualitativamente la mayoria de las tendencias observadas en el sistema de inversión de flujo, esclareciendo así algunos aspectos que no resultan inmediatos.

\subsection{Tiempo de ciclo}

Una de las variables más importantes en la operación de un sistema de inversión de flujo corresponde al tiempo de ciclo utilizado. Como fuera discutido anteriormente, debe ser lo suficientemente pequeño para que el frente de onda no penetre en el reactor al punto tal que la reacción se "apague". No obstante, si se utilizan tiempos de ciclo muy pequeños, en el caso del sistema convencional, se disminuirá la vida útil de las válvulas en forma proporcional y aumentará la contribución de la emisión de los VOCs alojados en la región de entrada al equipo al invertir el sentido de circulación.

Para este caso, se consideraron tiempos de ciclo en el rango comprendido entre 10 y 360 segundos y se evaluó la respuesta de ambos sistemas de inversión, el esquema convencional y el rotativo.

La respuesta del esquema convencional se muestra en la Figura 5.3, donde puede observarse un comportamiento térmico bien diferenciado según la fracción de inerte en el lecho. Para bajos valores de dicho parámetro (i.e., $f_{I} \lesssim 25 \%$ ), la temperatura máxima del lecho resulta prácticamente invariable 
en un amplio rango de $t_{\text {ciclo }}$. Por otro lado, a medida que se incrementa la fracción de inerte la temperatura máxima en el lecho aumenta y se vuelve más sensible al cambio en el tiempo del ciclo. Para elevados valores de $f_{I}$ (i.e., $75 \%$ ), la misma se vuelve significativamente sensible. Esto se halla en concordancia con resultados de otros autores, por ejemplo Matros y col. (1993).

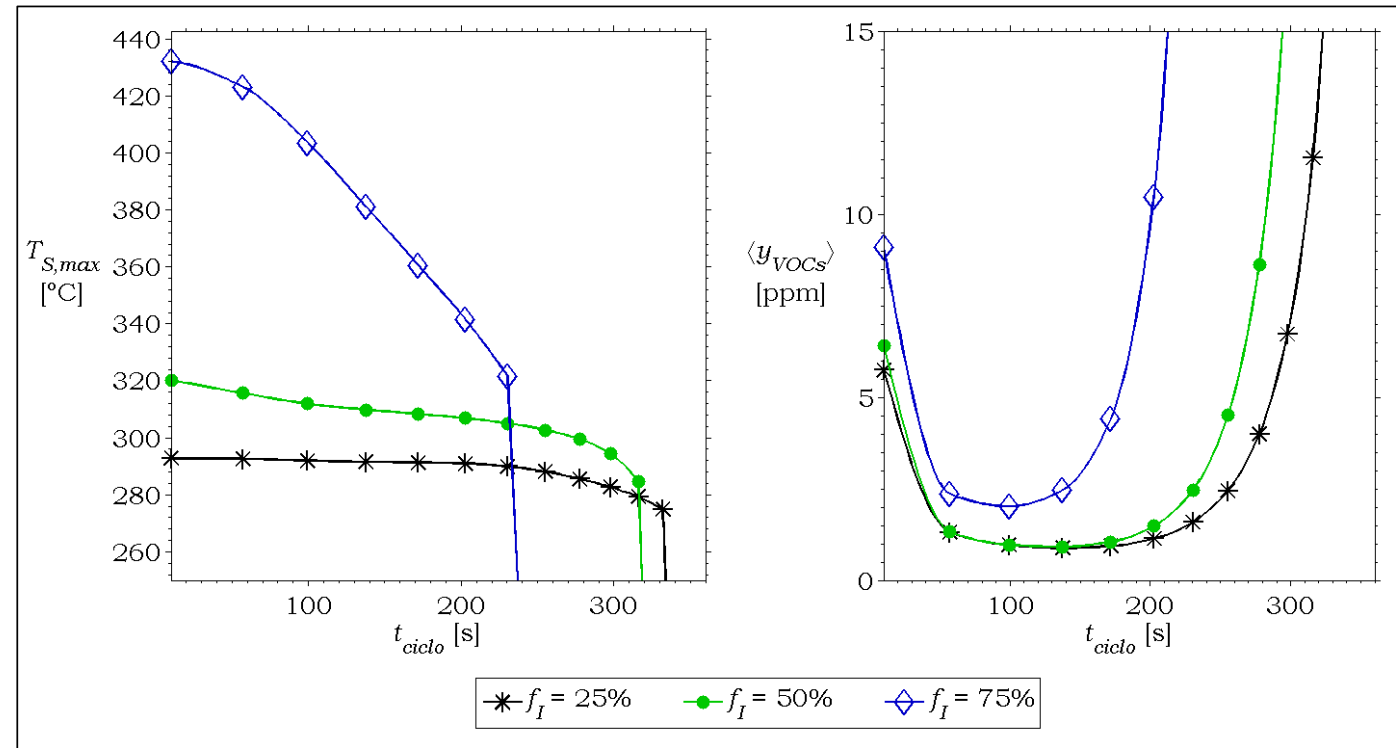

Figura 5.3: efecto del tiempo de duración del ciclo en el sistema convencional.

El cambio en la sensibilidad de la temperatura máxima con el tiempo de ciclo, entre bajos y altos $f_{I}$ (Figura 5.3), se puede comprender razonando en primera instancia en términos del RCC, el cual se corresponde con un tiempo de ciclo muy pequeño en el sistema de inversión de flujo. Como fuera discutido previamente, si en dicho sistema la fracción de inerte es nula (o pequeña), se formará una amplia meseta en donde la temperatura se mantendrá aproximadamente constante e igual a la máxima. Si ahora se piensa en una situación en la que $t_{\text {ciclo }}$ sea mayor, se deberá considerar el movimiento del frente de onda que se genera en la región de precalentamiento y se mueve en el sentido de avance de la corriente. Como la longitud efectiva de precalentamiento quedará determinada por la temperatura de ignición de los VOCs, es esperable que el aumento de $t_{\text {ciclo }}$ solo modifique la longitud de la meseta, sin cambiar apreciablemente el valor de $T_{S, \max }$. Esto último se puede apreciar en las Figuras $5.4 \mathrm{y}$ 5.5 , en donde se muestran los perfiles de temperatura en ambas fases y de fracciones molares en la fase gaseosa para diferentes tiempos. Adicionalmente, 


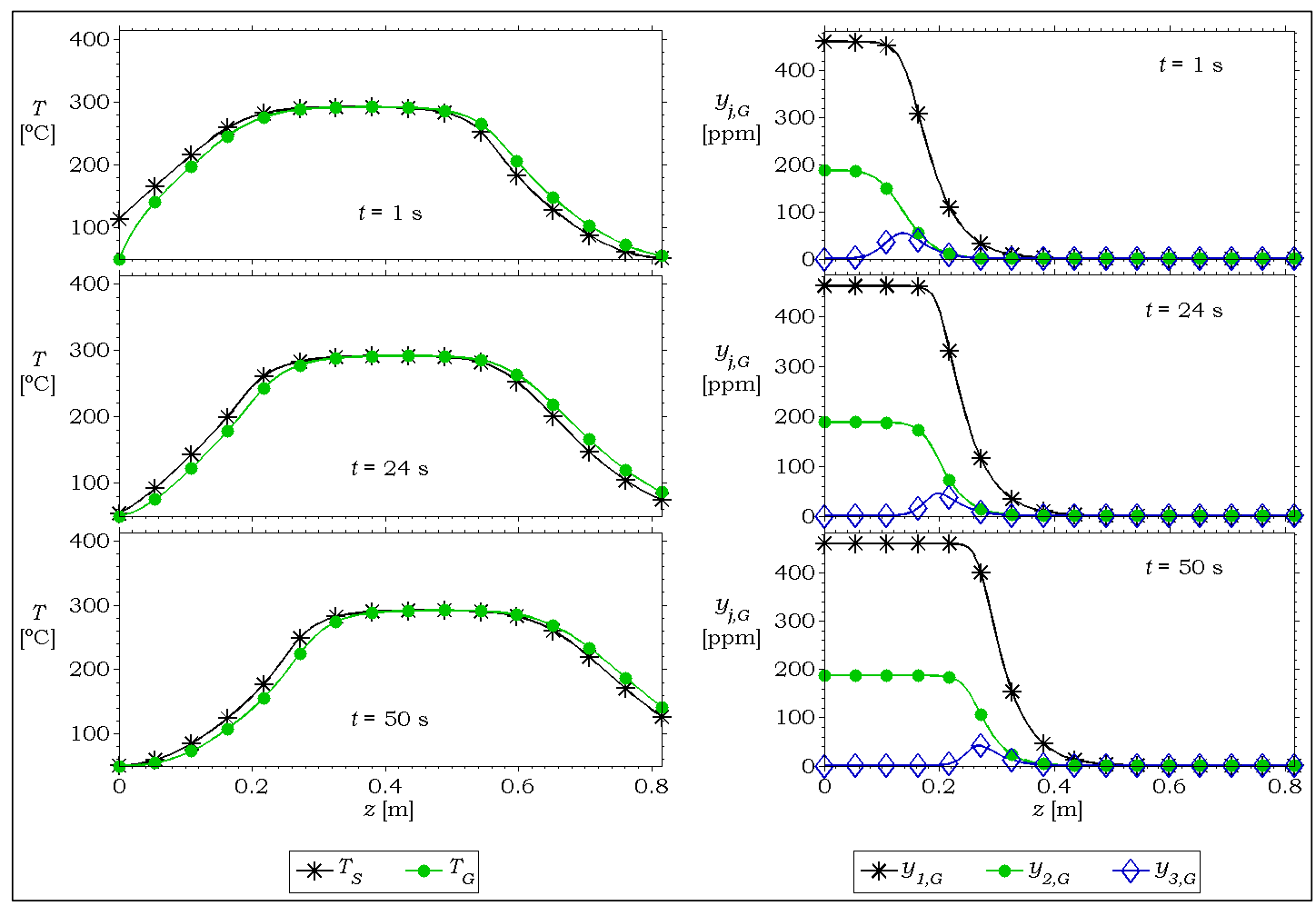

Figura 5.4: perfiles de temperatura y fracción molar en el sistema de inversión de flujo convencional sin inerte, para el css. Sentido de flujo: de izquierda a derecha $\left(t_{\text {ciclo }}=100 \mathrm{~s}\right)$.

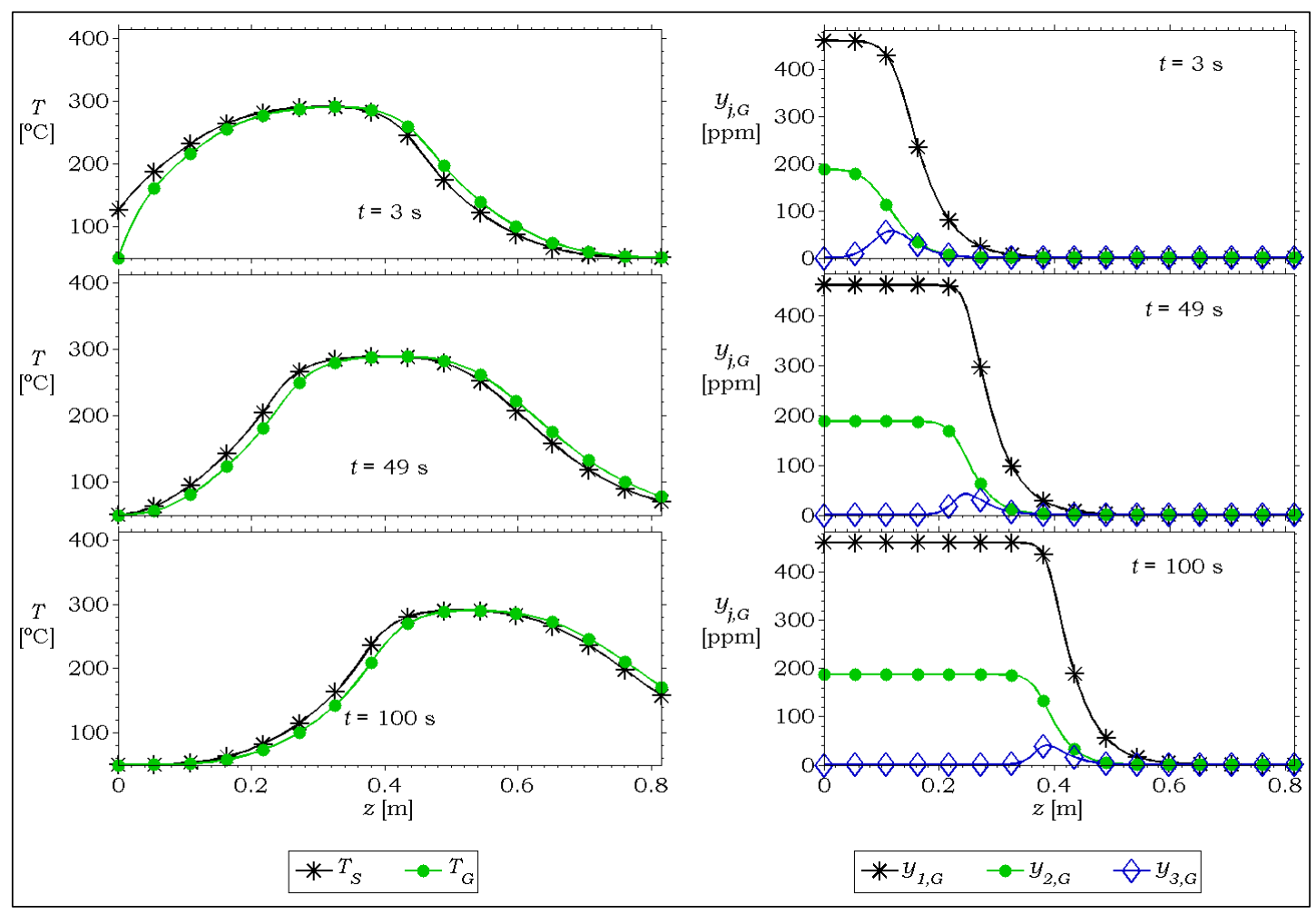

Figura 5.5: perfiles de temperatura y fracción molar en el sistema de inversión de flujo convencional sin inerte, para el css. Sentido de flujo: de izquierda a derecha $\left(t_{\text {ciclo }}=200 \mathrm{~s}\right)$. 
en la Figura 5.6 se condensan en un único gráfico, los perfiles de temperatura de la fase sólida y la fracción molar de acetato de etilo, a fin de visualizar con mayor facilidad el movimiento del frente de onda.

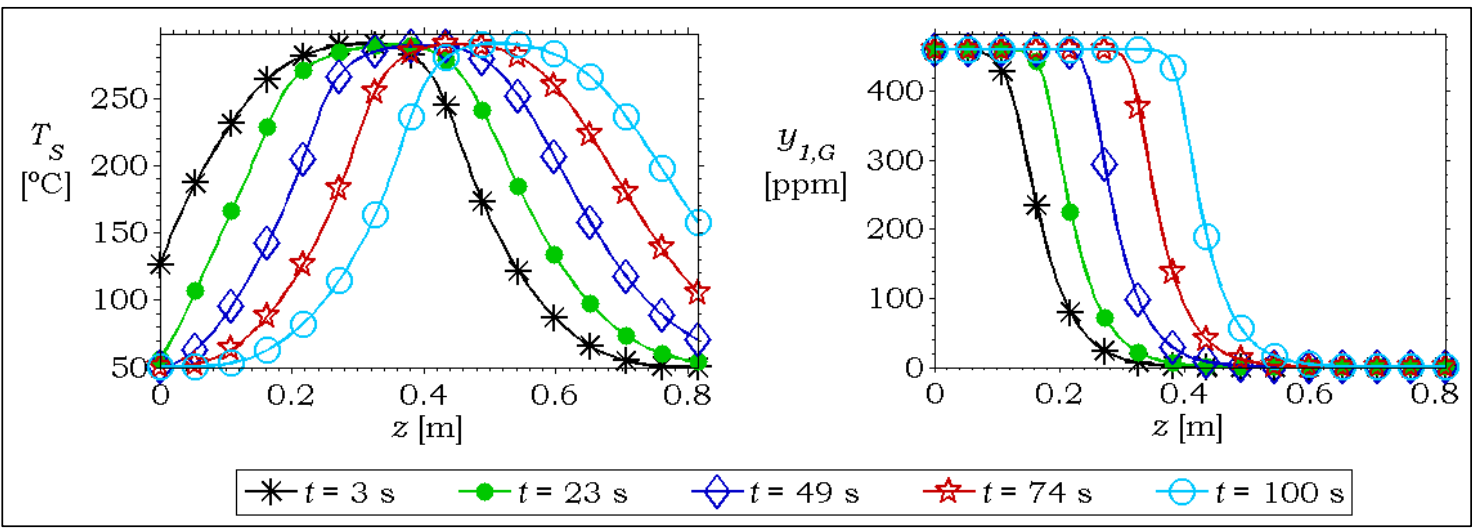

Figura 5.6: perfiles de temperatura en la fase sólida y de acetato de etilo en la fase gaseosa para diferentes tiempos. Sentido de flujo: de izquierda a derecha $\left(t_{\text {ciclo }}=200 \mathrm{~s}\right)$.

Por otro lado, a medida que la fracción de inerte aumenta, la temperatura máxima resulta más sensible al tiempo de ciclo empleado, debido a que la longitud de precalentamiento (promedio) variará casi proporcionalmente con $t_{\text {ciclo }}$. Esto se puede visualizar más fácilmente si se comparan situaciones con tiempos de ciclos bien diferenciados para $f_{I}=75 \%$ (ver Figuras 5.7 y 5.8). Si el tiempo de ciclo es pequeño (Figura 5.7), la longitud efectiva de la zona de precalentamiento corresponderá a la totalidad de la longitud inerte en el extremo donde la corriente está ingresando. Esto se debe a que, cuanto más pequeño sea $t_{\text {ciclo }}$, la temperatura de la fase sólida varía muy poco en todo el ciclo, acercándose al valor predicho mediante el RCC. Por el contrario, si el tiempo de ciclo es elevado (Figura 5.8), la totalidad del inerte será utilizado como zona de precalentamiento solo al inicio de cada semiciclo, mientras que el desplazamiento de la onda fría con el simultáneo enfriamiento del sólido en el extremo donde ingresa la corriente, reducirá la longitud efectiva de precalentamiento.

En cuanto a la cantidad de VOCs emitida, el comportamiento frente a la variación en el tiempo de duración del ciclo (Figura 5.3, derecha) responde a dos factores. En primer lugar, a bajos tiempos de inversión (ver Figuras 5.4 o 5.7) la onda no penetra en la región central del lecho, la cual se mantiene a una 


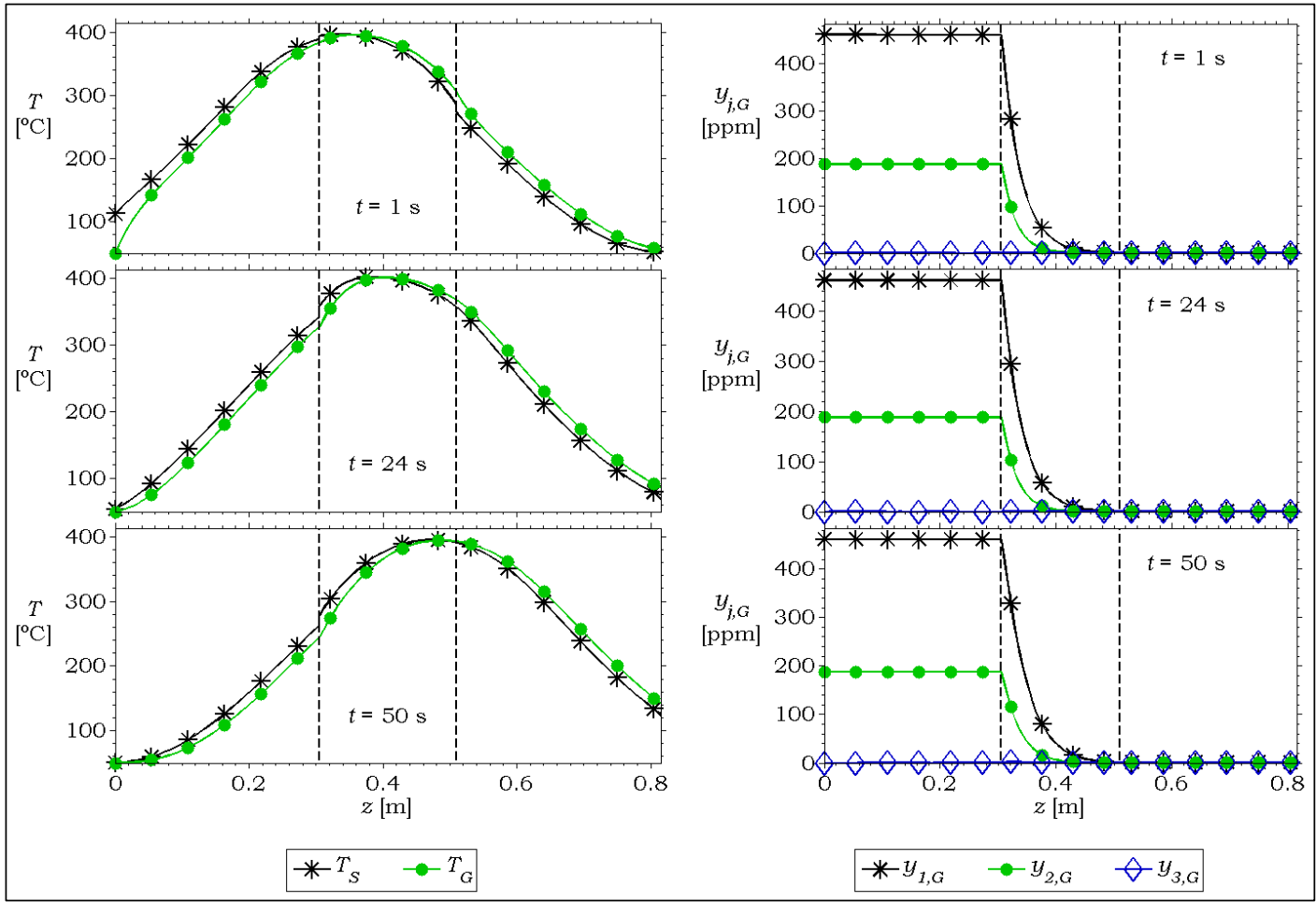

Figura 5.7: perfiles de temperatura y fracción molar en el sistema de inversión de flujo convencional con un 75\% de región inerte, para el css (las líneas punteadas verticales indican los límites inerte-catalizador). Sentido de flujo: de izquierda a derecha ( $\left.t_{\text {ciclo }}=100 \mathrm{~s}\right)$.

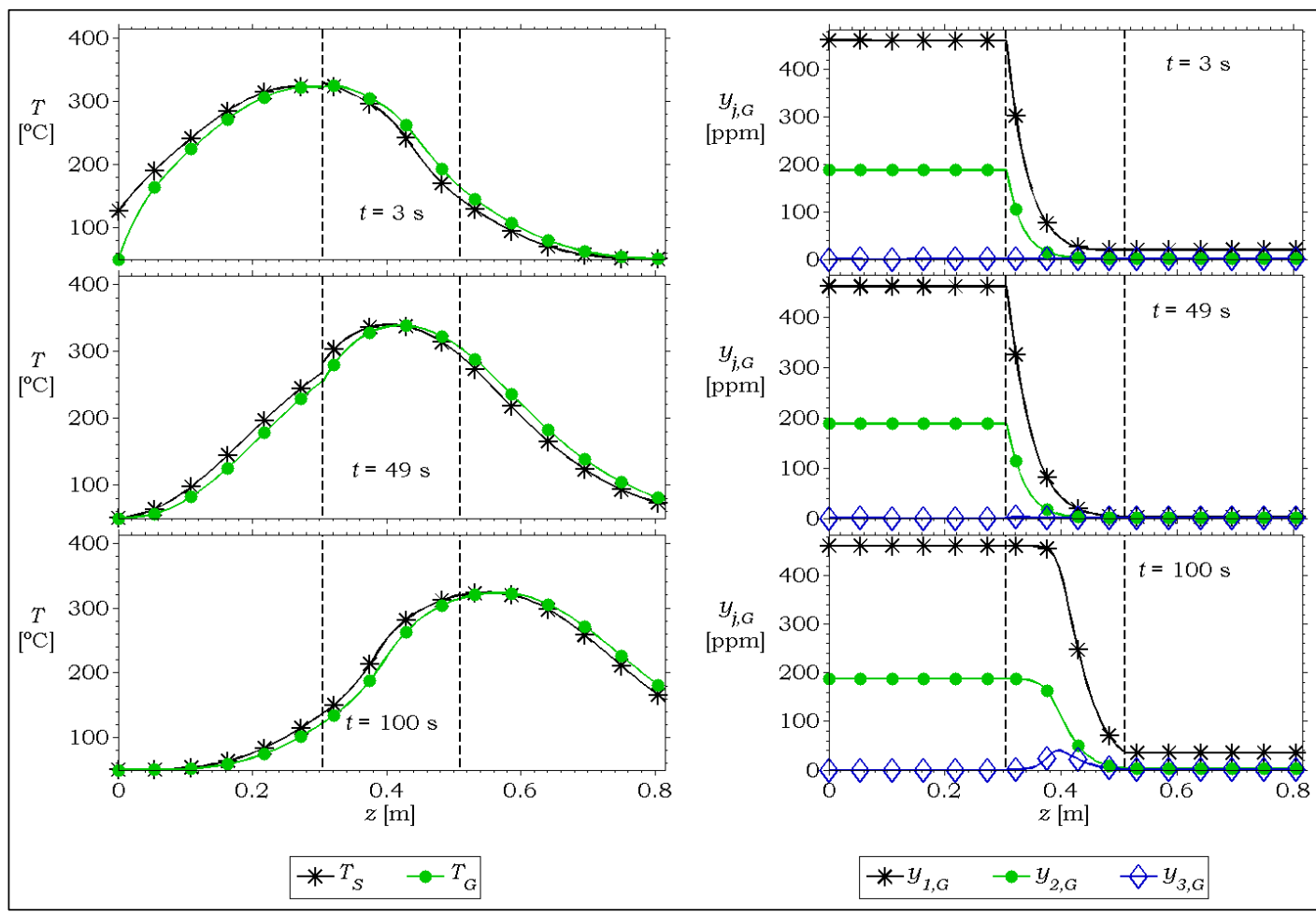

Figura 5.8: perfiles de temperatura y fracción molar en el sistema de inversión de flujo convencional con un 75\% de región inerte, para el css (las líneas punteadas verticales indican los limites inerte-catalizador). Sentido de flujo: de izquierda a derecha $\left(t_{\text {ciclo }}=200 \mathrm{~s}\right)$. 
temperatura elevada formándose la "meseta" típica de los sistemas de inversión de flujo (Matros y Bunimovich, 1996) y los VOCs que alcanzan dicha región son convertidos prácticamente por completo. Sin embargo, la emisión de los VOCs alojados en los extremos comienza a ser predominante cuando se opera en el sistema convencional. En segundo lugar, a altos tiempos de ciclo la onda penetra significativamente en el lecho (ver Figuras 5.5 o 5.8), aumentando rápidamente la emisión de VOCs, principalmente como consecuencia de la disminución del nivel térmico del lecho.

Para finalizar, en la Figura 5.9 se muestran los resultados del mismo estudio, realizado para el sistema de inversión de flujo rotativo (siendo la fracción de la sección transversal utilizada para la limpieza, $f_{L}=2 \%$ ).

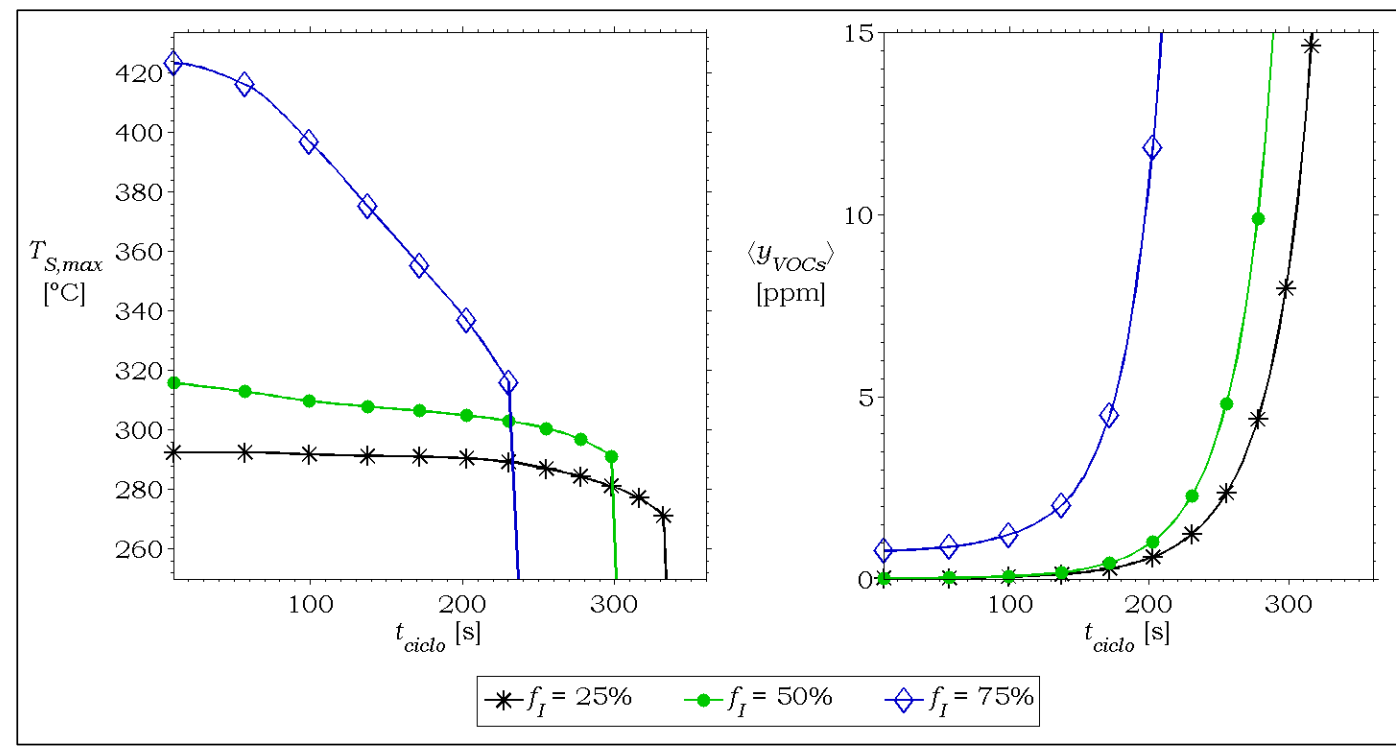

Figura 5.9: efecto del tiempo de duración del ciclo en el sistema rotativo $\left(f_{L}=2 \%\right)$.

En primer lugar, se puede observar que el comportamiento térmico del sistema convencional y del rotativo es prácticamente equivalente (comparar con la Figura 5.3), con la excepción de que en el último, la temperatura máxima es ligeramente inferior y se modifica muy levemente el valor de tiempo de ciclo para el cual el sistema se extingue. Esto se debe a que la velocidad másica es ligeramente superior en el sistema rotativo que en el convencional, debido a que una fracción de la sección total en el primero es utilizada para la limpieza de los canales. La principal diferencia se da en la emisión de VOCs, pudiendo apreciarse que el uso del sistema rotativo evita la expulsión del volumen de gas conteniendo VOCs (alojado en la región fría y/o inerte) al invertir el senti- 
do de circulación, tal como fuera mencionado en el Capítulo 3. De esta manera, se logran niveles de emisión prácticamente nulos para fracciones de inerte menores o iguales al 50\% y tiempos de ciclos tan pequeños como 10 segundos (en términos de la velocidad de rotación, $6 \mathrm{rpm}$ ).

Teniendo en cuenta entonces que la única diferencia significativa del uso del sistema rotativo se observa en la emisión de VOCs a bajos tiempos de ciclo (emisión prácticamente nula), en el resto del presente capitulo solo se estudiará el efecto de las variables seleccionadas anteriormente en el esquema convencional.

Por último, cabe destacar que el uso de tiempos de ciclos bajos quedará reservado exclusivamente para el sistema de inversión de flujo rotativo. En el Capítulo 7 se dará una discusión más detallada del rango de operación esperable de cada esquema.

\subsubsection{Comparación de los perfiles para $t_{\text {ciclo }}=10 \mathrm{~s}$ con los perfiles del RCC}

En el Capítulo 3 se enunció la similitud entre el RCC y la operación del reactor de inversión de flujo a bajos tiempos de ciclos. A fin de corroborar esto, en la Figura 5.10 se muestran los perfiles de temperatura en cada fase y de fracción molar de cada componente, para ambos sistemas (para el RCC se omiten los perfiles en la sección inferior). Se puede apreciar claramente la gran similitud entre ambos sistemas, tal como indican Nieken y col. (1995). No obstante, cabe aclarar que si el tiempo de ciclo se disminuye aún más, de forma tal que el tiempo del semiciclo sea sustancialmente menor que el tiempo de residencia de la corriente gaseosa, el sistema ya no alcanzará un css encendido debido a que la misma no dispone del tiempo necesario para pasar a través del reactor (Vanden Bussche y col., 1993). De todas maneras, entendiendo que esto carece de importancia práctica, se asumirá en general la equivalencia entre el sistema de inversión de flujo a $t_{\text {ciclo }} \rightarrow 0$ y el RCC. 


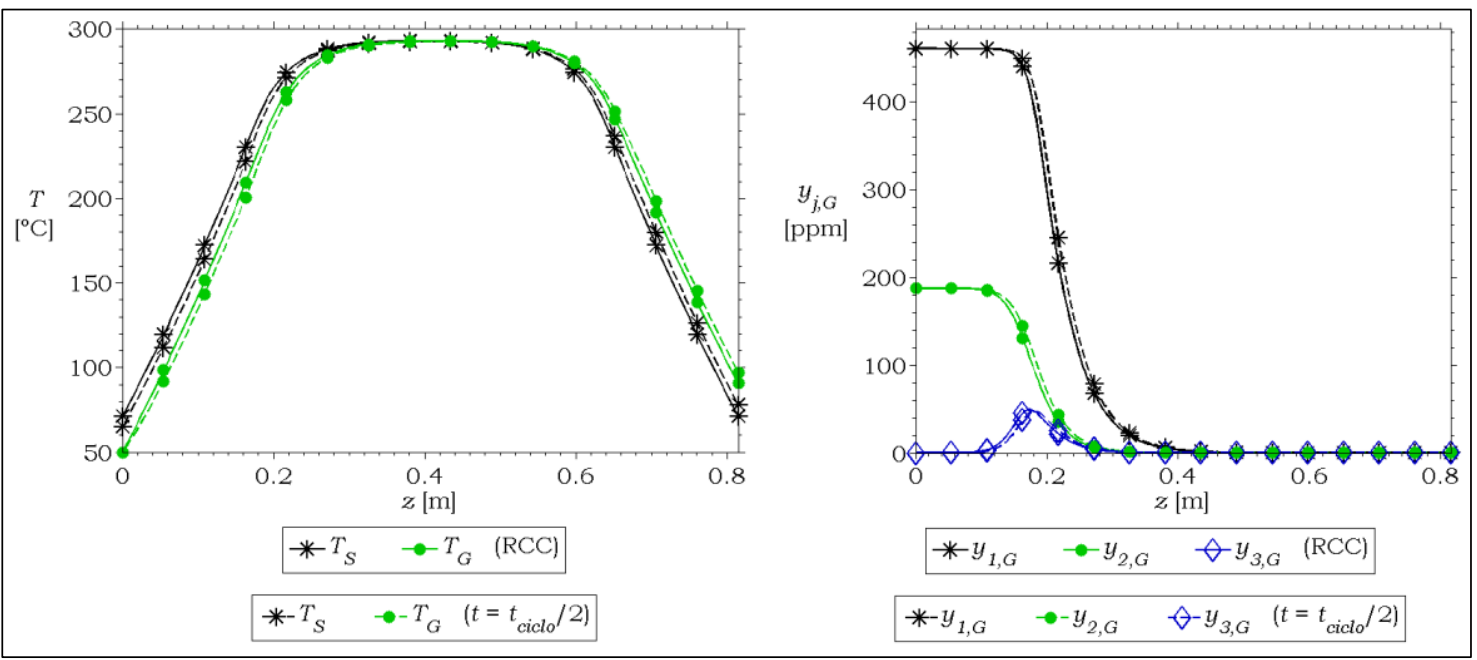

Figura 5.10: comparación entre los perfiles de temperatura y fracción molar según el RCC y el modelo completo. Sentido de flujo: de izquierda a derecha $\left(t_{\text {ciclo }}=10 \mathrm{~s}\right)$.

\subsection{Longitud del equipo}

Otro aspecto importante en este sistema es cómo influye la longitud total del equipo y si dicha influencia también dependerá de la fracción de inerte presente en el lecho. Para estudiar este aspecto, se consideró el rango comprendido entre 0.41 y $1.63 \mathrm{~m}$ (para el diámetro informado en la Tabla 5.1, dichos valores corresponden a un volumen total de lecho de 2 y $8 \mathrm{~m}^{3}$, respectivamente). La respuesta del sistema se muestra en la Figura 5.11. En la misma se puede observar nuevamente un comportamiento térmico bien diferenciado entre la configuración con una fracción de inerte baja o nula y una fracción elevada.

En primer lugar, para un dado tiempo de ciclo, existe un valor de longitud mínima para el cual el precalentamiento de la corriente resulta insuficiente y el sistema no alcanza un css encendido. Si bien este límite dependerá fuertemente del tiempo de ciclo utilizado, principalmente como consecuencia del movimiento del frente de onda, existirá un valor crítico que corresponde al caso en que $t_{\text {ciclo }} \rightarrow 0$. Luego, para una dada condición operativa, la longitud total del lecho no podrá ser menor que ese valor. Este aspecto será explorado con detalle en el Capitulo 7. 


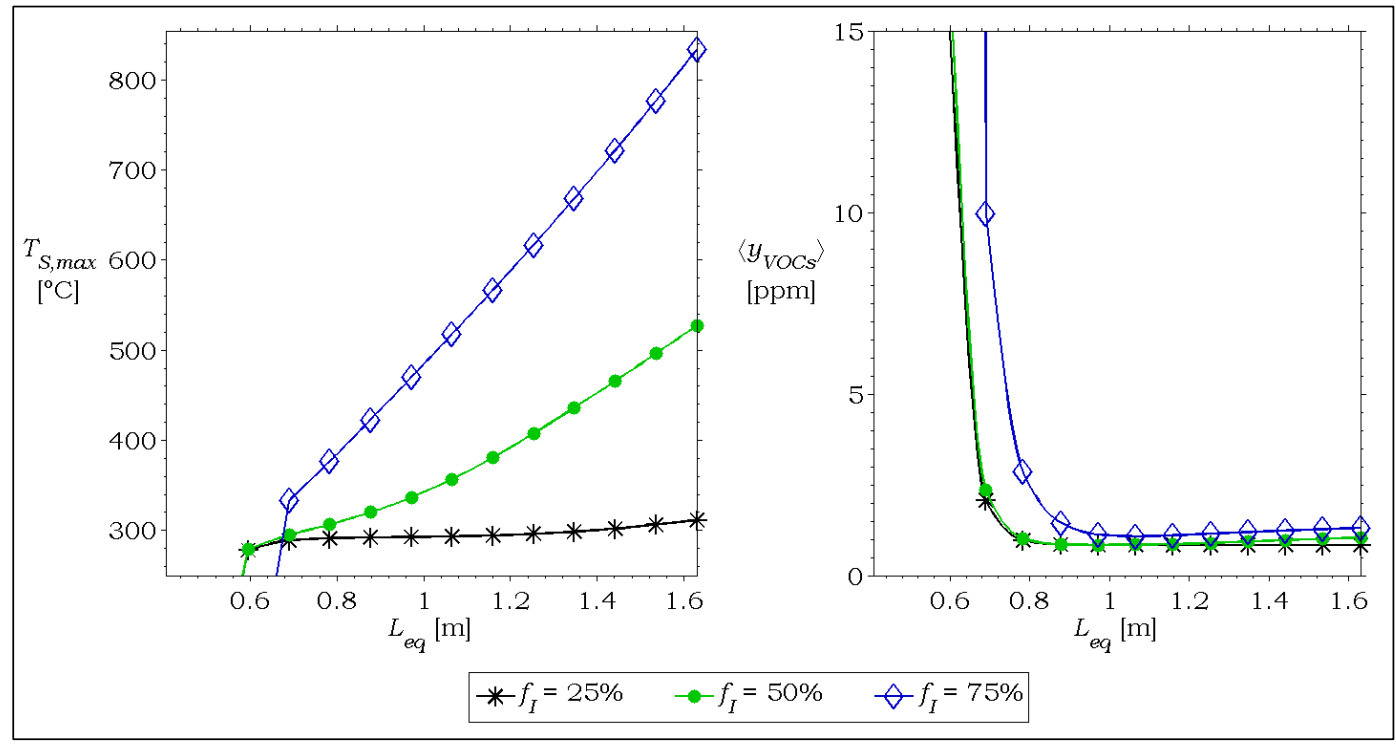

Figura 5.11: efecto de la longitud total del equipo en el sistema convencional $\left(t_{\text {ciclo }}=120 \mathrm{~s}\right)$.

Superada la longitud mínima necesaria para el tiempo de ciclo utilizado (en este caso, 120 segundos), la temperatura máxima en el lecho resulta prácticamente independiente de la longitud total para fracciones de inerte pequeñas (solo hay un ligero aumento a altos valores de longitud total para la configuración con $\left.f_{I}=25 \%\right)$. Por el contrario, cuando la fracción de inerte es elevada, la temperatura máxima resulta muy sensible a la longitud de lecho, exhibiendo un comportamiento aproximadamente lineal con la misma para valores elevados de longitud si $f_{I}=50 \%$ y en todo el rango si $f_{I}=75 \%$. Esto se debe básicamente al mismo fenómeno ya descrito en la sección anterior. Es decir, mientras la cantidad de inerte presente sea nula o muy baja, la región de precalentamiento (sin reacción química) quedará determinada por la longitud necesaria para alcanzar la temperatura de ignición de los VOCs. Como dicha región se mueve sin cambiar apreciablemente su forma, la temperatura máxima no varía significativamente si la longitud del lecho aumenta. Por otra parte, cuando la cantidad de inerte es significativa, la región de precalentamiento es, al menos durante un periodo de tiempo, directamente proporcional a la longitud de la región inerte? ${ }^{7}$ En ese caso, el aumento de la longitud total traerá aparejado un aumento en la longitud de precalentamiento, en forma proporcional a la fracción de inerte.

\footnotetext{
7 Dependiendo del tiempo de ciclo, si la onda formada penetrase apreciablemente en la región catalítica, la región de precalentamiento comenzaría a disminuir, tendiendo a adoptar la forma correspondiente al caso de baja fracción de inerte.
} 
De hecho, la aproximación (5.5) dada en la Sección 5.1 permite, pese a su simplicidad, explicar la tendencia observada. Por un lado, si la temperatura al final de la primera región inerte no supera la temperatura de ignición o si todo el lecho es catalítico, la temperatura máxima no dependerá de la longitud total del lecho. Por el otro, si la temperatura al final de dicha región supera a la de ignición, la temperatura máxima dependerá linealmente con la longitud total del lecho, con una pendiente que es proporcional a la fracción de inerte presente.

En cuanto a la emisión de VOCs, la misma permanece prácticamente inalterada al aumentar la longitud total del lecho, a excepción de un ligero incremento para las configuraciones con $f_{I}=50 \%$ y $f_{I}=75 \%$. Esto último es una consecuencia de la emisión de los VOCs alojados en la región inerte donde se alimenta la corriente.

La poca sensibilidad en la emisión de VOCs con la longitud total (superada la longitud mínima necesaria), es típica de los sistemas de inversión de flujo, en los cuales los css (estables) suelen caracterizarse por una conversión nula (estado apagado) o prácticamente completa (estado encendido), como se describe, por ejemplo, en Marin y col. (2010).

\subsection{Contenido total de VOCs}

El contenido total de VOCs es una de las variables de mayor relevancia en los sistemas autotérmicos en general, pues determina la fuerza impulsora, $\Delta T_{a d}$, para la transferencia. De hecho, en muchos trabajos donde se intenta establecer mecanismos de diseño simplificados para este tipo de sistemas, este parámetro posee un rol central (e.g., Marín y col., 2009).

En este caso, se evaluó la respuesta del sistema frente a la variación en el contenido total de VOCs en el rango de $\Delta T_{a d}$ comprendido entre 20 y $60{ }^{\circ} \mathrm{C}$, la cual se muestra en la Figura 5.12.

En primer lugar, se puede apreciar que existe un umbral de $\Delta T_{a d}$ por debajo del cual el css resultante es apagado, aunque dicho límite depende de las condiciones operativas y del volumen total. Por otro lado, se observa nuevamente un comportamiento térmico bien diferenciado entre las configuraciones con 
baja y alta proporción de inerte en el lecho. Para todos los casos, la tendencia observada resulta aproximadamente lineal con el aumento del $\Delta T_{a d}$, pero con pendientes que dependen de la fracción de inerte en el lecho. El análisis simplificado en términos del RCC, también permite explicar este comportamiento desde un punto de vista cualitativo. De hecho, la expresión (5.5) predice una tendencia lineal con $\Delta T_{a d}$, cuya pendiente varía en forma proporcional al valor de $f_{I}$ según: $1+\frac{a_{v} h_{m o d} f_{I} L_{e q}}{4 G c_{p G}}$.

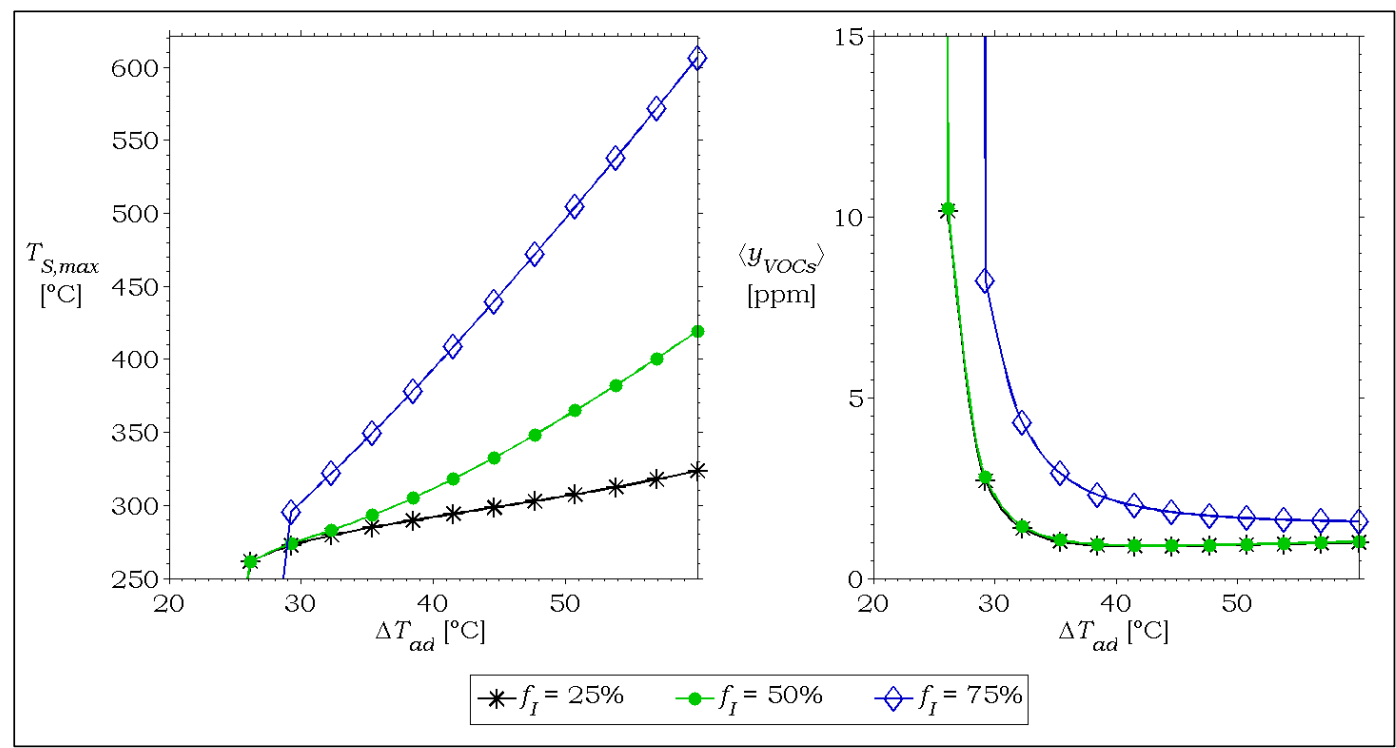

Figura 5.12: efecto del contenido total de VOCs en el sistema convencional (en términos del $\left.\Delta T_{\text {ad }} ; t_{\text {ciclo }}=120 \mathrm{~s}\right)$.

En lo que respecta a la emisión de VOCs, se observa un comportamiento similar al producido al variar la longitud total: una vez superado el valor umbral de $\Delta T_{a d}$, la emisión de VOCs permanece prácticamente inalterada, independiente de la fracción de inerte. Solo la curva para $f_{I}=75 \%$ muestra una variación ligeramente distinta, siendo decreciente en todo el rango. Esto se debe a que, con fracciones de inertes menores o iguales al 50\%, la temperatura en el sistema y la longitud catalítica son lo suficientemente elevadas para garantizar la destrucción total de los VOCs y el nivel de emisión queda determinado por la cantidad de VOCs alojada en los extremos fríos y/o inertes. Por el contrario, para $f_{I}=75 \%$, la longitud de la región catalítica no es suficiente y el descenso observado en la cantidad emitida responde al mayor grado de 
destrucción de los VOCs cuando el contenido de los mismos es mayor. Esto último ocurre debido a que el aumento de temperatura en este caso supera con creces la corta longitud de la región catalitica y el aumento en la cantidad de VOCs.

\subsection{Caída en la actividad del catalizador}

Como fuera dicho al comienzo del presente capítulo, el conocimiento del efecto que tiene las variables hasta aquí estudiadas, permite comprender mejor el sistema bajo análisis, lo cual presumiblemente derivará en criterios de diseño y/u operación más adecuados según el objetivo establecido.

En este contexto, también es importante conocer qué tan sensible es el sistema a la caída en la actividad del catalizador, debido a la diferencia en general entre los procesos de tratamiento de efluentes y los de tipo productivos. En los sistemas productivos, una de las alternativas más comunes para compensar la caída de la actividad catalítica es establecer un protocolo de variación de la temperatura de operación o, dependiendo de la aplicación, variar la carga al reactor dejando la temperatura constante, entre otras opciones (Bartholomew y Farrauto, 2006). Sin embargo, en los procesos de purificación, la única alternativa posible recaería en la temperatura de operación, que en el esquema estudiado en este capítulo, podría variarse a través de la modificación del tiempo de ciclo (dependiendo de la fracción de inerte).

Para estudiar el efecto de la caída en la actividad del catalizador sobre la operación del sistema de inversión de flujo, se asumió en primer lugar que la vida útil del catalizador es significativamente mayor al tiempo de ciclo. En segundo lugar, considerando que aquí solo interesa conocer el efecto que la caída en la actividad del catalizador tiene sobre el estado de operación del sistema y no cómo es el tipo y velocidad de desactivación, se vinculó la caída en la actividad a una disminución en el factor pre-exponencial de cada coeficiente cinético. Por último, se asumió que la desactivación del catalizador es la misma en cualquier posición del lecho.

De acuerdo a lo descrito en el párrafo anterior, se consideró una variación en la relación entre la actividad del catalizador en un momento dado y la actividad del catalizador fresco, $a / a_{0}$, entre 1 y $10^{-3}$. Los resultados se muestran 
en la Figura 5.13, donde se puede apreciar un comportamiento atípico en la temperatura máxima del lecho: la misma aumenta a medida que la actividad del catalizador disminuye, hasta que esta última alcanza un valor crítico por debajo del cual el sistema se apaga. Esta tendencia característica del sistema de inversión de flujo, pareciera ser completamente contradictoria con la disminución de la actividad catalítica, a tal punto que ha sido llamado como "comportamiento erróneo" por algunos autores (e.g., Eigenberger y Nieken, 1988).

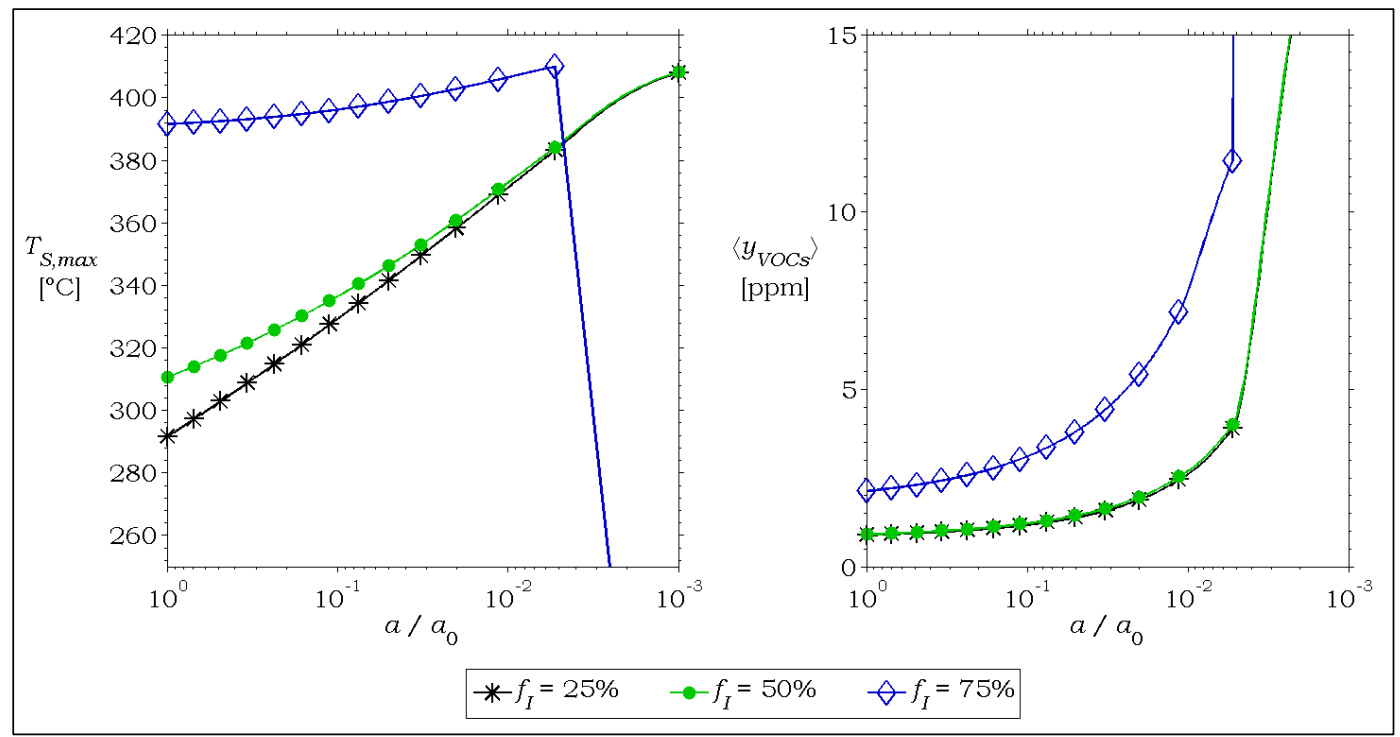

Figura 5.13: efecto de la caída en la actividad del catalizador en el sistema convencional $\left(t_{\text {ciclo }}=120 \mathrm{~s}\right)$.

Sin embargo, lo anterior se explica con los conceptos ya discutidos en este capítulo. Teniendo en cuenta que la caída en la actividad catalítica puede vincularse a un aumento en la temperatura de ignición, se puede ver a partir de la aproximación (5.5) que la longitud de precalentamiento aumentará (debido a que el catalizador también participa del intercambio de energía en forma regenerativa). Como consecuencia, el sistema tiende a adaptarse automáticamente, aumentando la temperatura en el lecho y consiguiendo con ello velocidades de reacción nuevamente elevadas. No obstante, la longitud disponible para la reacción disminuye, y no se logra compensar por completo la disminución de la actividad, por lo cual aumenta la emisión de VOCs, como se observa en el gráfico derecho de la Figura 5.13. De todas maneras, este aumento es prácticamente imperceptible para valores de actividad de hasta diez veces menores 
que los niveles del catalizador fresco. En la práctica, este valor resulta más que razonable como el máximo admisible de caída en la actividad catalítica, previo a su reemplazo o, de ser posible, su regeneración.

A los fines de clarificar la discusión del párrafo anterior, en la Figura 5.14 se muestran los perfiles de temperatura en la fase sólida y de acetato de etilo en la fase gaseosa, calculados mediante el RCC para 4 niveles de actividad. En la misma se observa claramente que a medida que la actividad disminuye, la reacción (y consecuentemente la liberación de energía) se desplaza hacia el centro del reactor. Como consecuencia, aumenta la región de precalentamiento y el sistema alcanza una temperatura más elevada.

Se debe reconocer que este comportamiento no se observa en un sistema autotérmico convencional, como por ejemplo uno formado por un intercambiador de superficie y un reactor catalítico, debido a que dicho sistema no posee la capacidad de adaptar el área de intercambio.

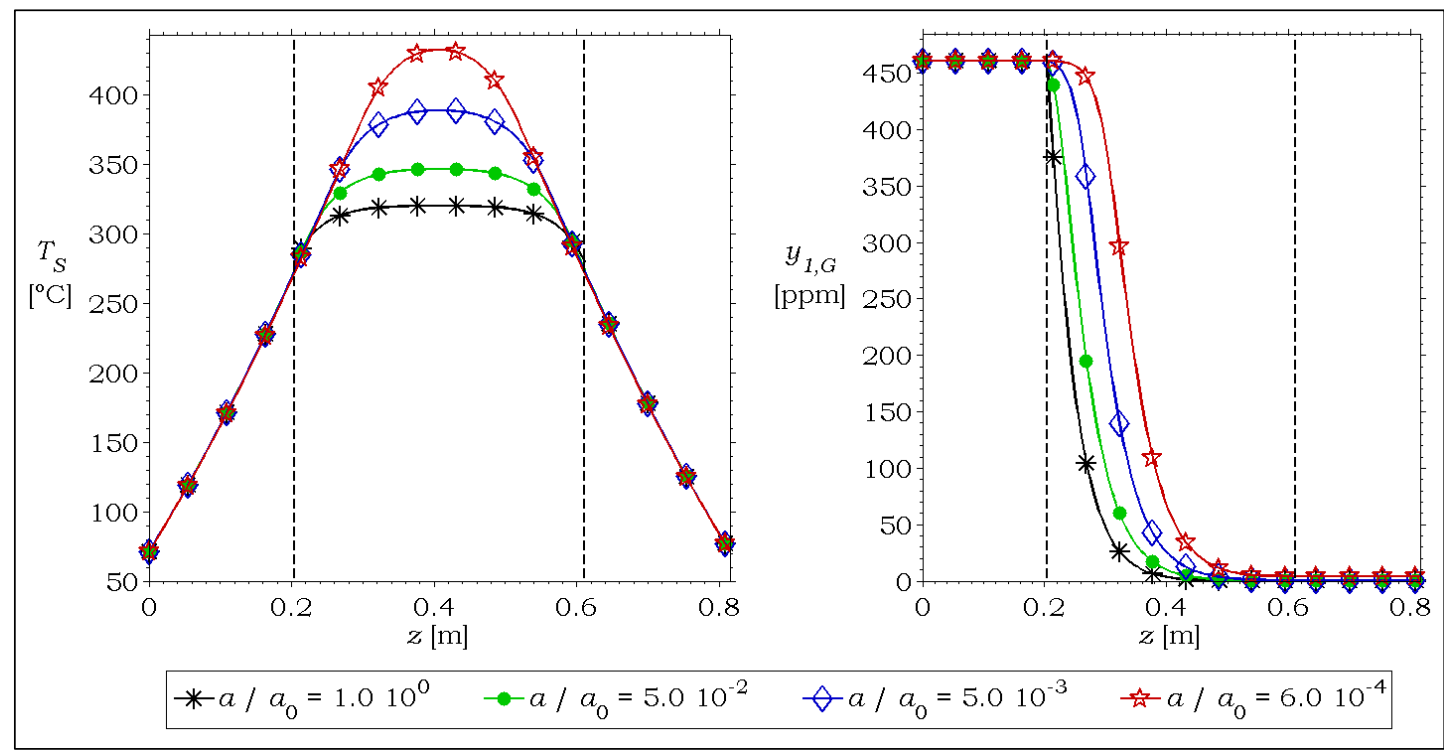

Figura 5.14: efecto de la caída en la actividad del catalizador según el modelo RCC $\left(f_{I}=50 \%\right)$.

\subsection{Aspectos adicionales}

Hasta aquí se ha estudiado el efecto de las variables más relevantes en el sistema, dejando de lado otras, ya sea porque es esperable que no varien en el proceso bajo análisis o su efecto sea muy poco significativo. En esta sección se 
discutirá brevemente el efecto de otras variables, con la intención de completar el panorama general brindado en este capítulo.

Una de las variables no consideradas es la temperatura de alimentación de la corriente, la cual presumiblemente no cambiará en más de unos $\sim 20^{\circ} \mathrm{C}$. No obstante, Matros (1989) estudió su efecto en un amplio rango, para una configuración sin inerte en los extremos, corroborando que su efecto sobre el estado de operación del sistema es prácticamente nulo (es decir, no se ve alterado ni $T_{S, \max }$ ni $\left.\left\langle y_{V O C S}\right\rangle\right)$, a menos que la misma sea comparable a la temperatura de ignición de los VOCs. Esto puede predecirse a partir de la expresión (5.5), la cual se reduce a $T_{S, \max } \approx \max \left\{T_{i g n} ; T_{G}^{0}\right\}+\Delta T_{a d}$, para $f_{I}=0$. De hecho, esta aproximación también permite estimar un efecto directamente proporcional de la temperatura de alimentación sobre la temperatura máxima, si la cantidad de inerte es muy elevada, situación en la cual $T_{i g n}$ será menor que $\vec{T}_{G}\left(L_{I}\right)$.

Otra variable que se ha omitido hasta aquí es la composición de VOCs en la corriente de alimentación para un dado $\Delta T_{a d}$. En base a lo discutido en las secciones precedentes (en particular sobre el efecto del $\Delta T_{a d}$ y de la actividad del catalizador), pareciera lógico suponer que la composición no afectará en forma significativa el estado del sistema mientras se mantenga el $\Delta T_{a d}$ constante. En particular, si se analizan los valores de los coeficientes cinéticos de las tres reacciones (ver Tabla 3.3) en el rango de 200 a $400{ }^{\circ} \mathrm{C}$, se encuentra que los mismos siguen la tendencia $\mathrm{AE}<\mathrm{Et}<\mathrm{Ac}$, para todo el rango. Luego, recordando el estudio sobre la actividad catalítica, es esperable que la mayor temperatura máxima se presente para el caso en que la alimentación solo contenga acetato de etilo.

Para completar esta discusión, en la Figura 5.15 se presentan los resultados de la variación de la composición manteniendo el $\Delta T_{a d}$ en el valor base de $40{ }^{\circ} \mathrm{C}$ y variando la relación de $y_{1, G}^{0} /\left(y_{1, G}^{0}+y_{2, G}^{0}\right)$ entre 0 y 1 . Como era de esperar, en la misma se observa que el efecto de la composición es muy leve, tanto en la temperatura máxima como en la emisión de VOCs.

En primer lugar, la variación de la temperatura máxima se explica, en principio, en base al análisis realizado en los párrafos anteriores. Sin embargo, 
hay un efecto interesante al aumentar la fracción de inerte en el lecho hasta valores muy elevados (i.e., $f_{I}=75 \%$ ). En ese caso, la temperatura máxima en el lecho pierde sensibilidad frente a la variación de la composición, respecto de los casos con una menor fracción de inerte. Esto puede deberse a las altas temperaturas encontradas en la configuración con $f_{I}=75 \%$, en donde es esperable que predomine la resistencia a la transferencia de materia y la cinética posea un rol secundario.

En segundo lugar, la emisión de VOCs no se ve alterada en forma significativa, aunque nuevamente aparece una diferencia de comportamiento entre la configuración con $f_{I}=75 \%$ y el resto.

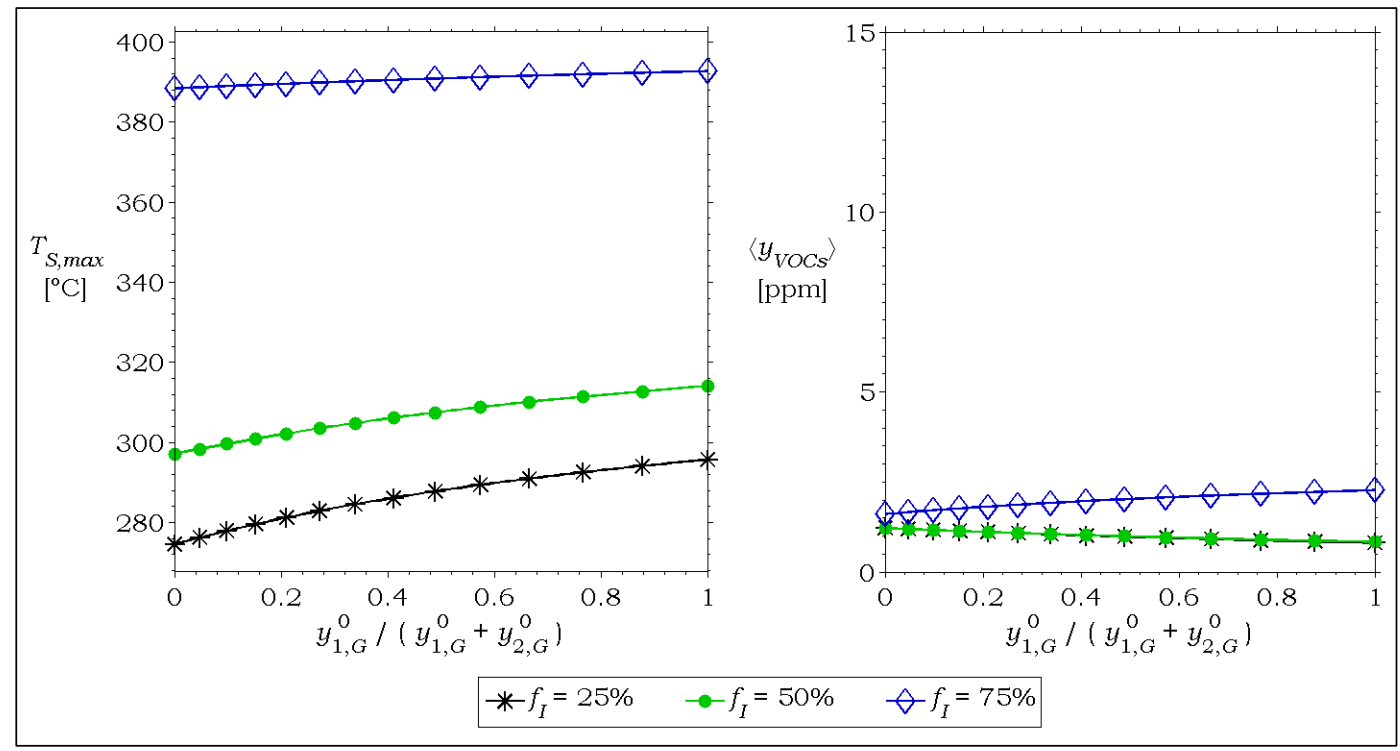

Figura 5.15: efecto de la composición de VOCs en la alimentación para $\Delta T_{a d}=40{ }^{\circ} \mathrm{C}$ $\left(t_{\text {ciclo }}=120 \mathrm{~s}\right)$.

Para explicar este fenómeno puede ser útil recordar que la emisión global de VOCs en el sistema convencional está fuertemente influenciada por la cantidad expulsada al invertir el sentido de circulación. Adicionalmente, se debe tener en cuenta que la relación entre las entalpías de combustión del acetato de etilo y del etanol es cercana a 2, por lo cual el contenido total de VOCs (molar) es mayor cuando toda la corriente contiene etanol. Luego, si se considera una situación extrema en la cual todos los VOCs que alcanzan la región catalíticamente activa reaccionan por completo, resulta inmediato que la emisión global (la cual estaría dada exclusivamente por la emisión al invertir) debe 
disminuir a medida que la proporción de acetato de etilo aumenta. Esto último es lo que se observa en la Figura 5.15 (derecha) para $f_{I} \leq 50 \%$.

Por el contrario, cuando la fracción de inerte es del 75\%, la emisión de VOCs sigue, llamativamente, un comportamiento inverso al anterior. No obstante, debe considerarse que en esta circunstancia ya no puede mantenerse la suposición realizada anteriormente (ver, por ejemplo, las Figuras 5.7 y 5.8) y la cantidad remanente de VOCs a la salida en la corriente comienza a tener más peso en la emisión global. Luego, el aumento observado en $\left\langle y_{\text {VOCs }}\right\rangle$ se debe a que la velocidad de reacción efectiva para el acetato de etilo, cuando el mismo es el único componente presente, es significativamente menor a la velocidad de reacción del etanol en las mismas condiciones.

\subsection{Conclusiones y comentarios finales}

En este capítulo se estudió la influencia de las principales variables en el estado de operación de los sistemas de inversión de flujo. Como tales se han considerado: la fracción de inerte en el lecho, el tiempo de ciclo, la longitud total del equipo, el contenido total de VOCs manteniendo la composición constante y, finalmente, la actividad catalitica.

Se ha utilizado el modelo del reactor en contra-corriente para estudiar de manera simplificada el efecto de la proporción de material inerte en el lecho. La misma posee una gran influencia en el comportamiento térmico del sistema, sin afectar significativamente la emisión de VOCs. En base a algunos razonamientos sencillos seguidos por otros autores, se desarrolló una expresión (Ec. 5.5), que permite explicar la diferencia de comportamiento al variar la fracción de inerte.

Los principios fundamentales que rigen el comportamiento del sistema de inversión de flujo guardan una estrecha relación con los del reactor en contra-corriente. Sin embargo, en los sistemas de inversión de flujo aparece otro concepto adicional: la formación y movimiento de un frente de onda térmico. En consecuencia, el comportamiento de este sistema queda determinado cualitativamente por la interacción entre el intercambio de calor y la liberación de energía por reacción química, así como por el movimiento del frente de onda. 
En particular, se corroboró que para bajos tiempos de ciclo, los perfiles del sistema de inversión de flujo adoptan la forma de los perfiles que estima el RCC.

De estos estudios se puede concluir que el tiempo de ciclo, la longitud del lecho y el contenido total de VOCs juegan un rol primordial, especialmente si se considera que el sistema posee una mayor sensibilidad a los parámetros mencionados cuando la fracción de inerte es elevada (i.e., $f_{I} \gtrsim 50 \%$ ) y que en la práctica es muy usual el reemplazo del material catalítico por otro inerte (e.g., Marin y col., 2010).

Se verificó que el sistema de inversión de flujo rotativo presenta un comportamiento térmico prácticamente idéntico al sistema convencional. Como fuera enunciado en el Capítulo 3, la ventaja principal de este sistema es que elimina el problema de la emisión de los VOCs alojados en la región inerte y/o fría del equipo (por donde se alimenta la corriente en un semiciclo dado) al invertir el sentido de circulación. Esto hace posible disminuir el tiempo de ciclo sin aumentar la emisión global de VOCs (aún para la configuración con $f_{I}=75 \%$ ), lo cual permitiría disminuir la longitud total del lecho en una etapa de diseño.

Se analizó el efecto de la caída de la actividad catalítica en el estado de operación del esquema de inversión de flujo convencional. Este sistema presenta un comportamiento atípico, "adaptándose" naturalmente a dicha caída de actividad, lo cual lleva a que la misma no posea un efecto adverso sobre la emisión de VOCs si dicho decaimiento se limita a una décima parte de la actividad del catalizador fresco, cualquiera sea la fracción de inerte utilizada (para el valor inicial elegido en la comparación). Este comportamiento "anómalo" se explicó nuevamente con conceptos introducidos mediante el RCC: la capacidad del sistema de modificar la extensión de la región de precalentamiento según la temperatura de ignición de los VOCs. De hecho, el RCC presenta un comportamiento completamente análogo, lo cual se ejemplificó mediante el estudio de la caída en la actividad para este último, con $f_{I}=50 \%$.

Finalmente, se mencionaron los principales efectos de la variación en la temperatura de alimentación y de la composición de VOCs para un $\Delta T_{a d}$ constante. Ninguna de estas variables influye marcadamente en el comportamiento del sistema de inversión de flujo, para las condiciones y el sistema reactivo analizado en el presente estudio. 


\section{Capítulo 06}

\section{- Análisis del sistema regenerador-reactor}

- Fracción de limpieza

- Caída en la actividad del catalizador 

En forma similar a lo expuesto en el Capítulo 5, se presenta aquí un análisis de la respuesta del sistema regenerador-reactor frente a variaciones en los parámetros operativos y de diseño más relevantes.

Previo al análisis, es útil resaltar una importante diferencia entre este sistema y cualquiera de los esquemas de inversión de flujo. Como fuera estudiado en el Capítulo 5, los sistemas de inversión de flujo presentan una característica muy distintiva, que es su capacidad de "adaptar" la longitud efectiva de la zona de precalentamiento, sin la necesidad de introducir ningún tipo de modificación en la operación. Esto es posible debido a que el material catalítico también participa del intercambio regenerativo de energía. Se debe recordar que dicho comportamiento trae aparejado respuestas singulares frente a la variación de algunos parámetros, dándole cierta "flexibilidad natural". Un claro ejemplo de ello es el aumento de la temperatura máxima en el lecho y la prácticamente nula variación de la emisión de VOCs, a medida que la actividad catalítica disminuye. Un análisis cualitativo previo, permite concluir fácilmente que en el sistema regenerador-reactor no se encontrarán respuestas de ese tipo, ya que el lecho catalítico no participa del intercambio de energía regenerativo, sino que se comporta como un reactor adiabático convencional.

En este sistema la capacidad de intercambio queda determinada únicamente por la longitud del intercambiador rotativo y por el tiempo de ciclo utilizado. De hecho, debido a la operación estacionaria del reactor una vez que se ha alcanzado el css, el comportamiento del sistema es muy similar al de uno convencional formado por un intercambiador de superficie (por ejemplo, de tipo compacto) y el mismo reactor. La principal diferencia es que, en el esquema aquí estudiado, se podrá variar la capacidad de intercambio de energía una vez instalados los equipos, modificando el tiempo de duración del ciclo. Un aumento de este último parámetro se traducirá en una disminución de la capacidad de intercambio (disminuye la "eficiencia térmica del equipo") y viceversa (Willmott, 2002). De hecho, a muy bajos tiempos de ciclo (pero aún mayores que el tiempo de residencia del gas), el comportamiento térmico del regenerador se aproxima al de un intercambiador de calor en contra-corriente, representando esta situación un límite (superior) en la capacidad de intercambio de energía. Este aspecto será abordado con mayor detalle en el Apéndice 6.1. 
Aun cuando el efecto de la variación del tiempo de ciclo es conocido (al menos cualitativamente), se debe tener presente que el mismo representa una variable operativa de suma importancia (al igual que en los sistemas de inversión de flujo, es la única variable operativa una vez instalados los equipos). Por esta razón, es importante estudiar qué impacto posee en el sistema, sabiendo que el mismo fijará, para una dada longitud del regenerador, la temperatura de alimentación al reactor.

Por otro lado, una condición operativa que naturalmente se modifica es la actividad catalítica. Recordando que el esquema no posee una capacidad natural para adaptarse a dicho cambio (aspecto que, como fuera mencionado en los párrafos iniciales, lo diferencia de los sistemas de inversión de flujo), resulta importante evaluar su efecto sobre el estado de operación.

En adición, un carácter distintivo del regenerador, para este tipo de aplicación, es la fracción de la sección total destinada a la limpieza de los canales. A diferencia del esquema de inversión de flujo rotativo, la alimentación a la región de limpieza es independiente de la corriente principal, por lo cual es esperable que su impacto sobre la pérdida de carga total sea menor (al menos siempre que la velocidad másica en dicha etapa resulte menor o igual a la de las regiones de enfriamiento y calentamiento). Esto justifica su inclusión en el presente capítulo, en lugar de su estudio como parte de la etapa de diseño.

Como consecuencia de lo explicado anteriormente, el análisis en este capítulo solo se centra en el efecto de:

* el tiempo de duración del ciclo,

* la fracción de la sección total del regenerador utilizada para la limpieza, $f_{L}$

* la caída en la actividad del catalizador.

En todos los casos, se consideró el tiempo de ciclo como un parámetro (en forma similar a lo realizado con la fracción de inerte en el sistema de inversión de flujo), lo que permite ver el impacto del mismo sobre la temperatura de alimentación al reactor y de esta sobre la operación del sistema. Los datos generales para realizar el análisis se muestran en la Tabla 6.1, donde $L_{\text {Reg }} \mathrm{y}$ 
$D_{\text {Reg }}$ son la longitud y diámetro del regenerador respectivamente, $L_{R}$ y $D_{R}$ son la longitud y diámetro del reactor respectivamente, $N_{C}$ el número de celdas (canales) por unidad de superficie transversal, $\delta_{T}$ el espesor total de tabique, $t_{\text {ciclo }}$ el tiempo de duración del ciclo y $\Delta T_{a d}$ es el máximo salto térmico adiabático (se mantiene la proporción de acetato de etilo y etanol informada en el Capítulo 2). Cabe resaltar que se consideró en todos los casos $f_{L}+f_{E}=1 / 2$, siendo $f_{L}$ la fracción de la sección total del regenerador utilizada para la limpieza y $f_{E}$ la fracción que corresponde a la sección de enfriamiento del sólido (ver Figura 3.2).

Tabla 6.1: parámetros utilizados para el estudio del sistema regenerador-reactor.

\begin{tabular}{|c|c|c|c|c|c|c|c|}
\hline \multicolumn{2}{|c|}{ Regenerador } & \multicolumn{2}{|c|}{ Reactor } & \multicolumn{2}{|c|}{ Ambos equipos } & \multirow{2}{*}{$\begin{array}{c}\boldsymbol{t}_{\text {ciclo }} \\
{[\mathbf{s}]}\end{array}$} & \multirow[b]{2}{*}{$\begin{array}{r}\Delta \boldsymbol{T}_{a d} \\
{\left[{ }^{\circ} \mathbf{C}\right]}\end{array}$} \\
\hline $\begin{array}{r}L_{R e g} \\
{[\mathrm{~m}]}\end{array}$ & $\begin{array}{r}D_{R e g} \\
{[\mathrm{~m}]}\end{array}$ & $\begin{array}{c}L_{R} \\
{[\mathrm{~m}]}\end{array}$ & $\begin{array}{c}D_{R} \\
{[\mathrm{~m}]}\end{array}$ & $\begin{array}{c}\boldsymbol{N}_{\mathrm{c}} \\
{\left[\mathbf{m}^{-2}\right]}\end{array}$ & $\begin{array}{c}\delta_{T} \\
{[\mathrm{~m}]}\end{array}$ & & \\
\hline 0.519 & 2.5 & 0.570 & 1.768 & $3.110^{5}$ & $3.17510^{-4}$ & {$[10 ; 40 ; 80 ; 100 ; 120]$} & 40 \\
\hline
\end{tabular}

Se excluye nuevamente la velocidad másica, la cual se fijó para obtener una adecuada pérdida de carga ( $\lesssim 0.1$ bar para todos los casos), considerando adicionalmente que la relación entre la sección transversal total del regenerador y la del reactor es de 2. Para un caudal másico total dado, esto define inmediatamente el diámetro de ambos equipos. Estos aspectos serán abordados con mayor detalle en el Capítulo 8.

Por otro lado, no se analizan en forma detallada las variaciones en la longitud del intercambiador o en la cantidad total de VOCs, pues su influencia resulta directa en este sistema (definido el tiempo de ciclo, el esquema no difiere significativamente de uno convencional). Un aumento en la longitud del intercambiador derivará siempre en un aumento en la temperatura de alimentación al reactor y consecuentemente en una disminución en la cantidad remanente de VOCs (incremento de la conversión). Un mayor contenido de VOCs a la entrada poseerá dos efectos simultáneos. Por un lado, suponiendo que la conversión en el reactor es completa, aumentará el $\Delta T_{a d}$ y consecuentemente aumentará la temperatura de alimentación al mismo. Por otro lado, para el sistema reactivo presentado en el Capítulo 3, la mayor concentración 
de los VOCs favorecerá a la velocidad de reacción de cada componente. Esto se debe a una mayor elevación de temperatura en el reactor (aún si no se modificase la temperatura de alimentación al mismo).

\subsection{Fracción de limpieza}

Si bien la fracción de la sección total del equipo utilizada para la limpieza de los canales (abreviadamente, fracción de limpieza) no es uno de los parámetros con mayor impacto en el comportamiento del equipo, resulta interesante evaluar su impacto. Para ello, se consideró el rango de $f_{L}$ comprendido entre 0 ( $\sin$ fracción de limpieza) y 3\% de la sección total del regenerador (sujeto siempre a $\left.f_{L}+f_{E}=1 / 2\right)$. La respuesta obtenida se muestra en la Figura 6.1, en donde se han considerado como variables de "salida" más relevantes al contenido total de VOCs remanente, $\left\langle y_{\text {VOCS }}\right\rangle$, y a la temperatura de alimentación al reactor $T_{G}^{0, R}$.

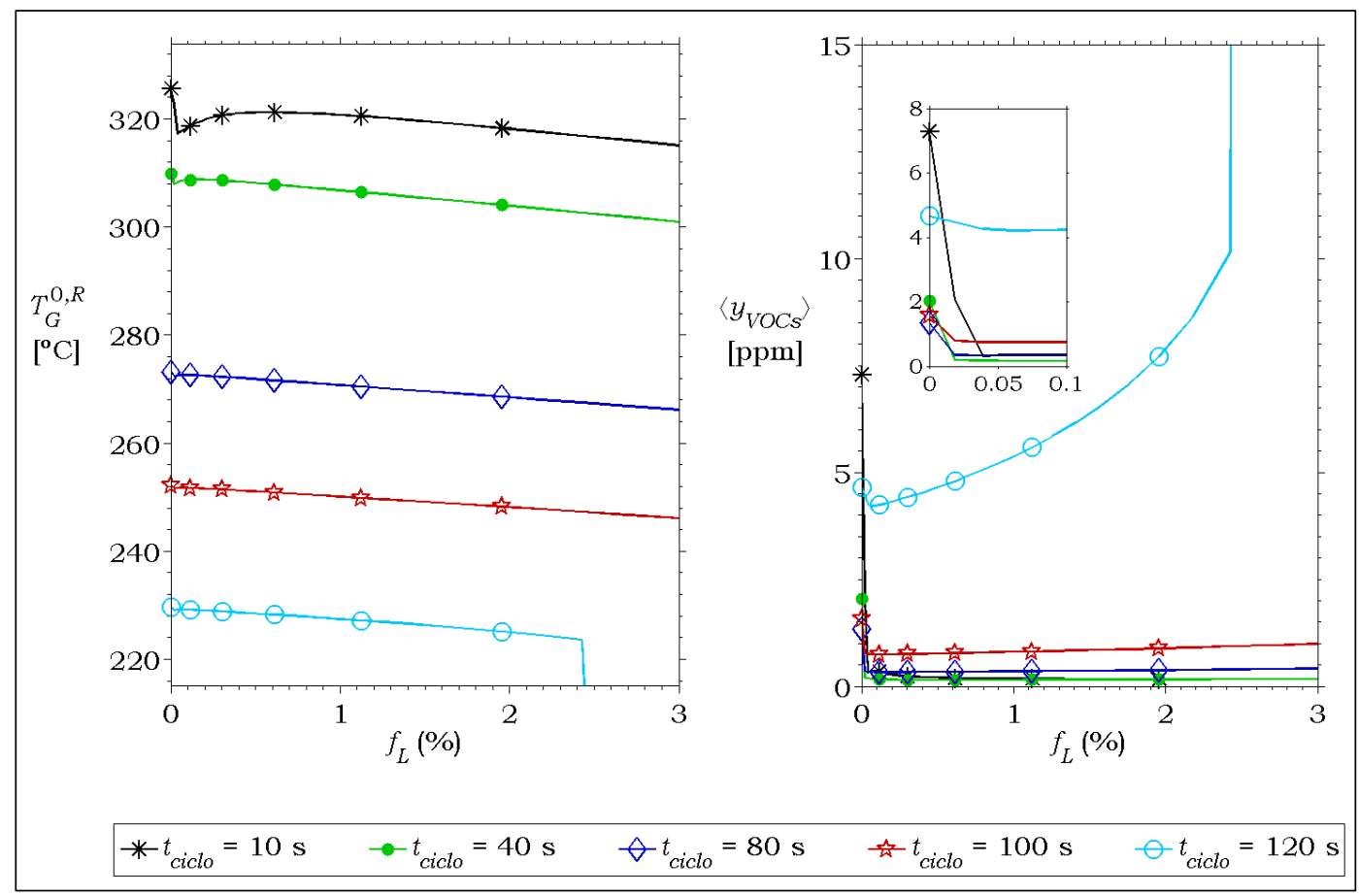

Figura 6.1: efecto de la fracción de limpieza en el sistema regenerador-reactor.

En primer lugar, cabe aclarar que el primer punto de cada curva $\left(f_{L}=0\right)$, corresponde al caso en que no existe fracción de limpieza y, lógicamente, tam- 
poco circula un caudal destinado para tal fin. Por esta razón, se aprecia una discontinuidad para $f_{L}=0$ en la temperatura de alimentación al reactor (más evidente en la curva para $\left.t_{\text {ciclo }}=10 \mathrm{~s}\right)$.

En segundo lugar, se puede notar la presencia de un máximo local en la temperatura de alimentación al reactor (claramente más evidente en la curva para $t_{\text {ciclo }}=10 \mathrm{~s}$, pero presente en todos los casos). Para entender esto, es conveniente remitirnos en primera instancia a las Ecs. (4.10) y (4.13), que determinan el caudal de limpieza mínimo, $G_{m, L, m i n}$, y el real, $G_{m, L}$. Recordando que el tiempo de la etapa de limpieza, $t_{E L}$, queda determinado por $t_{E L}=f_{L} t_{\text {ciclo }}$ y habiendo fijado $f_{L}+f_{E}=1 / 2$, la Ec. (4.10) se puede reescribir como:

$$
G_{m, L, \min }=\frac{S_{\text {tot }} \varepsilon_{L, I}}{t_{\text {ciclo }}} \int_{0}^{L_{\text {Reg }}} \rho_{G}\left[T_{G}\left(\left(1 / 2-f_{L}\right) t_{\text {ciclo }}, z\right)\right] d z
$$

Luego, mientras $f_{L}$ adopte valores pequeños $\left(f_{L} \leq 3 \%\right.$, de acuerdo al rango considerado en el presente estudio) es esperable que $\rho_{G}\left[T_{G}\left(\left(1 / 2-f_{L}\right) t_{\text {ciclo }}, z\right)\right]$ no varie apreciablemente entre una condición y otra, siendo únicamente una función de la posición. De verificarse esto último, el caudal de limpieza debería ser constante para un dado tiempo de ciclo. En la Tabla 6.2 se presentan los valores calculados del caudal de limpieza para cada valor de $t_{\text {ciclo }}$ (promedios), habiendo verificado que la variación entre los caudales de limpieza para diferentes valores de $f_{L}$ es, en todos los casos, del orden del 1\%o. Adicionalmente, se presenta en la misma el valor aproximado de $f_{L}$ en donde ocurre el máximo estacionario en la temperatura de alimentación al reactor, $f_{L}^{*}$, y la relación $G_{m, L} /\left[2\left(G_{m, L}+G_{m, t o t}\right)\right]$, cuyo significado quedará identificado posteriormente, donde $G_{m, t o t}$ representa el caudal másico de la corriente a tratar.

Teniendo presente que el caudal de limpieza es prácticamente independiente de $f_{L}$, la presencia del máximo se puede explicar fácilmente. En primer lugar, si la fracción de limpieza es muy pequeña, la velocidad másica del aire utilizado en esta región será muy elevada y, en consecuencia, circulará por el canal sin haber incrementado suficientemente su temperatura. Simultánea- 
mente, el comportamiento térmico de la corriente a tratar en la región de enfriamiento del sólido no habrá cambiado apreciablemente respecto al sistema sin limpieza. Por otro lado, si el valor de $f_{L}$ es elevado, la velocidad másica del aire de limpieza será baja y su temperatura a la salida del regenerador se aproximará a la temperatura del sólido en tal posición. Sin embargo, como se ha fijado $f_{L}+f_{E}=1 / 2$, la velocidad másica de la corriente a tratar en la región de enfriamiento será mayor y comenzará a disminuir su temperatura de salida. En consecuencia, es esperable que la temperatura de alimentación al reactor sea mayor cuando estos efectos se compensen y las velocidades másicas sean comparables.

Tabla 6.2: valores del caudal de limpieza, fracción de limpieza para el máximo local en $T_{G}^{0, R}$ y de la relación entre el caudal de limpieza y el total en la región de calentamiento del sólido, para los diferentes tiempos de ciclo empleados.

\begin{tabular}{cccc}
\hline $\begin{array}{c}\boldsymbol{t}_{\text {ciclo }} \\
{[\mathbf{s}]}\end{array}$ & $\mathbf{G}_{\boldsymbol{m}, \boldsymbol{L}}$ para $\boldsymbol{f}_{\boldsymbol{L}} \neq \mathbf{0}$ & $\boldsymbol{f}_{\boldsymbol{L}}^{*}$ & $\frac{\mathbf{1}}{\mathbf{2}} \frac{\boldsymbol{G}_{\boldsymbol{m}, \boldsymbol{L}}}{\mathbf{G}_{\boldsymbol{m}, \boldsymbol{L}}+\mathbf{G}_{\boldsymbol{m}, \text { tot }}}$ \\
\hline $\mathbf{[ \mathbf { k g } / \mathbf { s } ]}$ & $1.63910^{-1}$ & 0.585 & 0.636 \\
\hline 40 & $4.32310^{-2}$ & 0.156 & 0.169 \\
\hline 80 & $2.32610^{-2}$ & 0.081 & 0.091 \\
\hline 100 & $1.92310^{-2}$ & 0.069 & 0.075 \\
\hline 120 & $1.65010^{-2}$ & 0.057 & 0.065 \\
\hline
\end{tabular}

Asumiendo que se conoce el valor de $G_{m, L}$ para un dado tiempo de ciclo, se puede estimar la posición del máximo estacionario con relativa sencillez, exigiendo la igualdad en las velocidades másicas en cada región. Es decir:

$$
\frac{G_{m, t o t}}{f_{E}}=\frac{G_{m, L}}{f_{L}}=\underbrace{\begin{array}{c}
\text { región de } \\
\text { limpieza }
\end{array}}_{\begin{array}{c}
\text { región de } \\
\text { enfriamiento }
\end{array}}=\underbrace{\frac{G_{m, L}+G_{m, t o t}}{1-f_{E}-f_{L}}}_{\begin{array}{c}
\text { región de } \\
\text { calentamiento }
\end{array}}
$$


Luego, resolviendo las Ecs. (6.2), se obtiene: $f_{E}+f_{L}=1 / 2$ (lo que justifica la elección de estos valores) y $f_{L}=G_{m, L} /\left[2\left(G_{m, L}+G_{m, t o t}\right)\right]$. Esta última relación es la volcada en la Tabla 6.2, donde se puede apreciar que predice con razonable precisión la ubicación del máximo local en $T_{G}^{0, R}$.

Por último, en términos de la emisión total de VOCs, al igual que lo observado en el sistema de inversión de flujo rotativo, la presencia de una región destinada a la limpieza de los canales permite evitar la emisión de los VOCs retenidos en el volumen de aire alojado en los canales previos a la posición donde se invierte el sentido de circulación de la corriente en el regenerador (observar los diferentes puntos con $f_{L}=0$, comparados al resto de los valores -ver ampliación insertada en la Figura 6.1-). La excepción a este comportamiento ocurre para la curva con $t_{\text {ciclo }}=120 \mathrm{~s}$, como consecuencia de la cercanía al limite entre el css encendido y el apagado (de hecho, para dicha curva el css resulta apagado si la fracción de limpieza es mayor a $\approx 2.4 \%$ ).

\subsection{Caida en la actividad del catalizador}

Como fuera mencionado con anterioridad, este sistema no posee la capacidad de "adaptar" la capacidad de transferencia de la zona de precalentamiento, aspecto que si se verifica en los sistemas de inversión de flujo (para ambos esquemas propuestos). Esta carencia posiciona al conjunto regenerador-reactor en desventaja, al menos en principio y en relación a la cantidad de material involucrado (inerte y catalítico). Cabe preguntarse entonces si es factible encontrar condiciones de operación/diseño que permitan alcanzar un cierto grado de flexibilidad en términos de la desactivación del catalizador, comparable a lo observado en los sistemas de inversión de flujo. Para ello, se consideró una variación en la relación entre la actividad del catalizador en un momento dado y la actividad del catalizador fresco, $a / a_{0}$, entre $1 \mathrm{y} 10^{-3}$, para diferentes tiempos de ciclo. Los resultados se muestran en la Figura 6.2.

Como puede observarse en la Figura 6.2, mientras el css resulta encendido, la temperatura de entrada al reactor permanece prácticamente invariable frente a la variación en la actividad del catalizador. En cada caso existe un umbral de actividad (que llamaremos actividad crítica) para el cual el estado encendi- 
do no puede mantenerse sin introducir alguna modificación en la operación y por ende el sistema se apaga ${ }^{8}$. Adicionalmente, la transición entre un estado y otro es, en todos los casos, marcadamente abrupta. Esto es una consecuencia directa de la operación con elevada conversión de VOCs en el reactor en dicha situación. En este aspecto, los esquemas analizados en el presente estudio son semejantes: debido a la pequeña fuerza impulsora para la operación autotérmica (el $\left.\Delta T_{a d}\right)$ y la elevada energía de activación de las reacciones involucradas, los estados de operación posibles se caracterizan por una conversión prácticamente nula (css apagado) o esencialmente completa (css encendido).

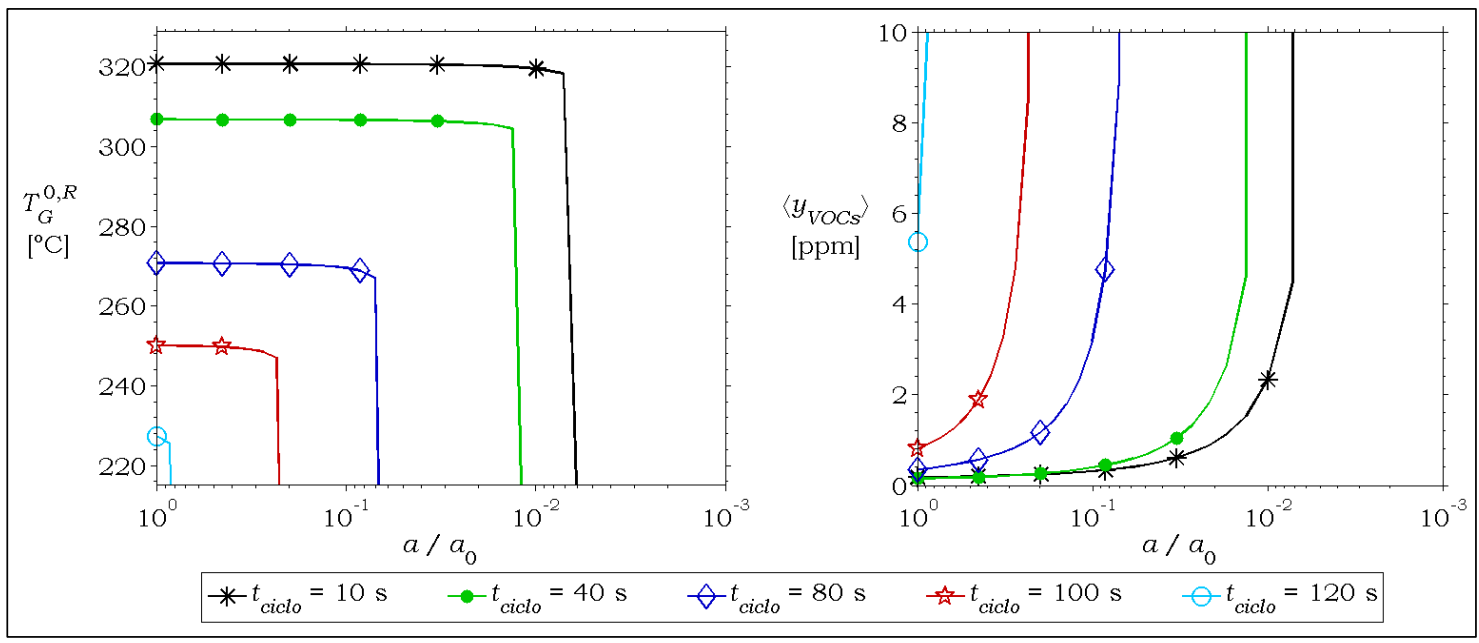

Figura 6.2: efecto de la caída en la actividad del catalizador en el sistema regenerador-reactor $\left(f_{L}=1 \%\right)$.

Adicionalmente, puede observarse que el umbral de actividad catalitica que separa los estados encendido y apagado, depende fuertemente de la temperatura de alimentación al reactor (en este caso, modificada a través del tiempo de ciclo). Por el contrario, como se desprende de los resultados del análisis realizado para los sistemas de inversión de flujo (Figura 5.13), en este último no existe tal sensibilidad o bien no resulta relevante si se consideran los bajos valores de actividad crítica alcanzados.

Lo señalado en el párrafo anterior es una consecuencia directa del tipo de esquema propuesto: una vez alcanzado el css, el reactor opera en forma adiabática y en estado estacionario, como en cualquier sistema convencional.

\footnotetext{
8 Cabe señalar que la emisión de VOCs en tal condición podría superar el valor límite establecido en el Capítulo 2 (9 ppm). No obstante, el conocimiento de la actividad crítica (definida como el valor umbral previo al apagado del sistema) sirve para establecer un marco de referencia.
} 
Luego, es de esperar que la temperatura de alimentación al mismo posea un fuerte impacto sobre el estado de operación, independientemente de cómo se haya alcanzado la misma. Para corroborar esto último, se buscó el valor de actividad crítica (con una tolerancia relativa de $10^{-3}$ ) para cuatro valores de longitud de regenerador $(0.494 \mathrm{~m}, 0.519 \mathrm{~m}, 0.545 \mathrm{~m}, 0.570 \mathrm{~m})$, y considerando un rango de $t_{\text {ciclo }}$ comprendido entre los 10 y los 100 segundos. Todos los demás parámetros corresponden a los informados en la Tabla 6.1.

Los resultados obtenidos se muestran en la Figura 6.3, donde se han registrado los valores de la actividad crítica, $\left(a / a_{0}\right)_{\text {crit }}$, la temperatura de alimentación al reactor y la cantidad de VOCs remanentes en dicha condición. En la misma se observa que un aumento en el tiempo de ciclo, o una menor longitud del regenerador, produce un descenso en la temperatura de alimentación al reactor en la condición crítica. Consecuentemente, el valor de actividad crítica aumenta (situación más desfavorable) al incrementar el tiempo de ciclo.

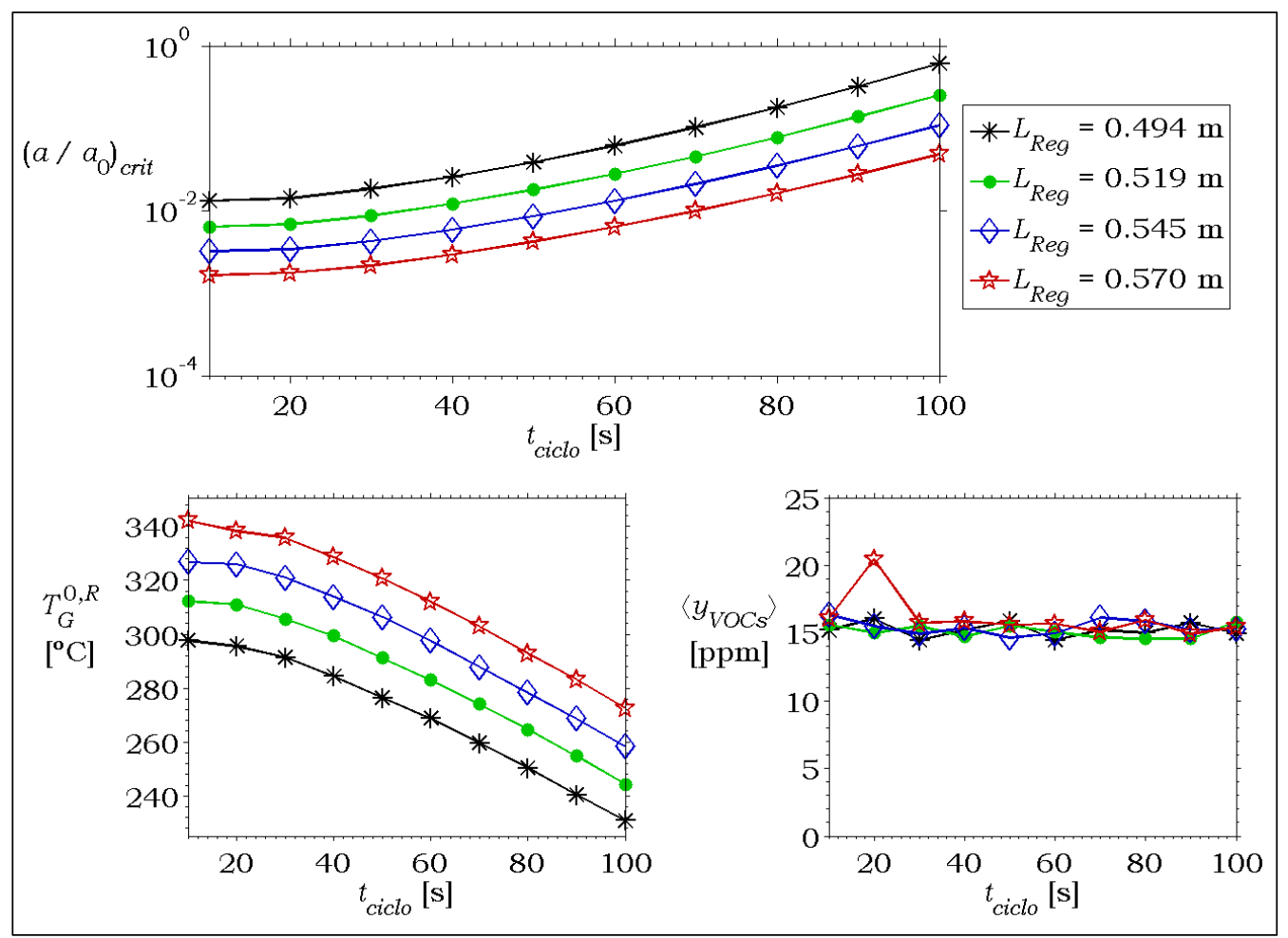

Figura 6.3: efecto del tiempo de ciclo y la longitud del regenerador sobre la actividad crítica del catalizador $\left(f_{L}=1 \%\right)$.

Por otro lado, la cantidad de VOCs emitida en la condición crítica resulta aproximadamente constante y muy pequeña para todas las condiciones (cer- 
cana a unos $15 \mathrm{ppm})$. Esto es una característica general de los esquemas autotérmicos cuando se procesan reacciones de oxidación, cuyas energías de activación son típicamente muy elevadas, y la temperatura de alimentación al sistema es significativamente menor a la temperatura de reacción. Este aspecto se aborda desde una perspectiva simplificada en el Apéndice 6.1.

Todo lo discutido en los párrafos anteriores se puede visualizar eliminando el tiempo de ciclo como parámetro y graficando directamente los valores de $\left(a / a_{0}\right)_{c r i t}$ contra la temperatura de alimentación al reactor (para cada longitud de regenerador). Esto se muestra en la Figura 6.4, en donde se observa claramente que la dependencia de $\left(a / a_{0}\right)_{c r i t}$ con el tiempo del ciclo o la longitud del regenerador se debe exclusivamente a la variación de la temperatura de alimentación al reactor (todas las curvas se solapan en una sola).

De esta manera, operando el sistema de forma tal de obtener una temperatura de alimentación al reactor suficientemente elevada (por ejemplo, en el rango comprendido entre los 260 y $\operatorname{los} 300^{\circ} \mathrm{C}$ ), se alcanza un grado de flexibilidad comparable con los que ofrecen ambos esquemas de inversión de flujo.

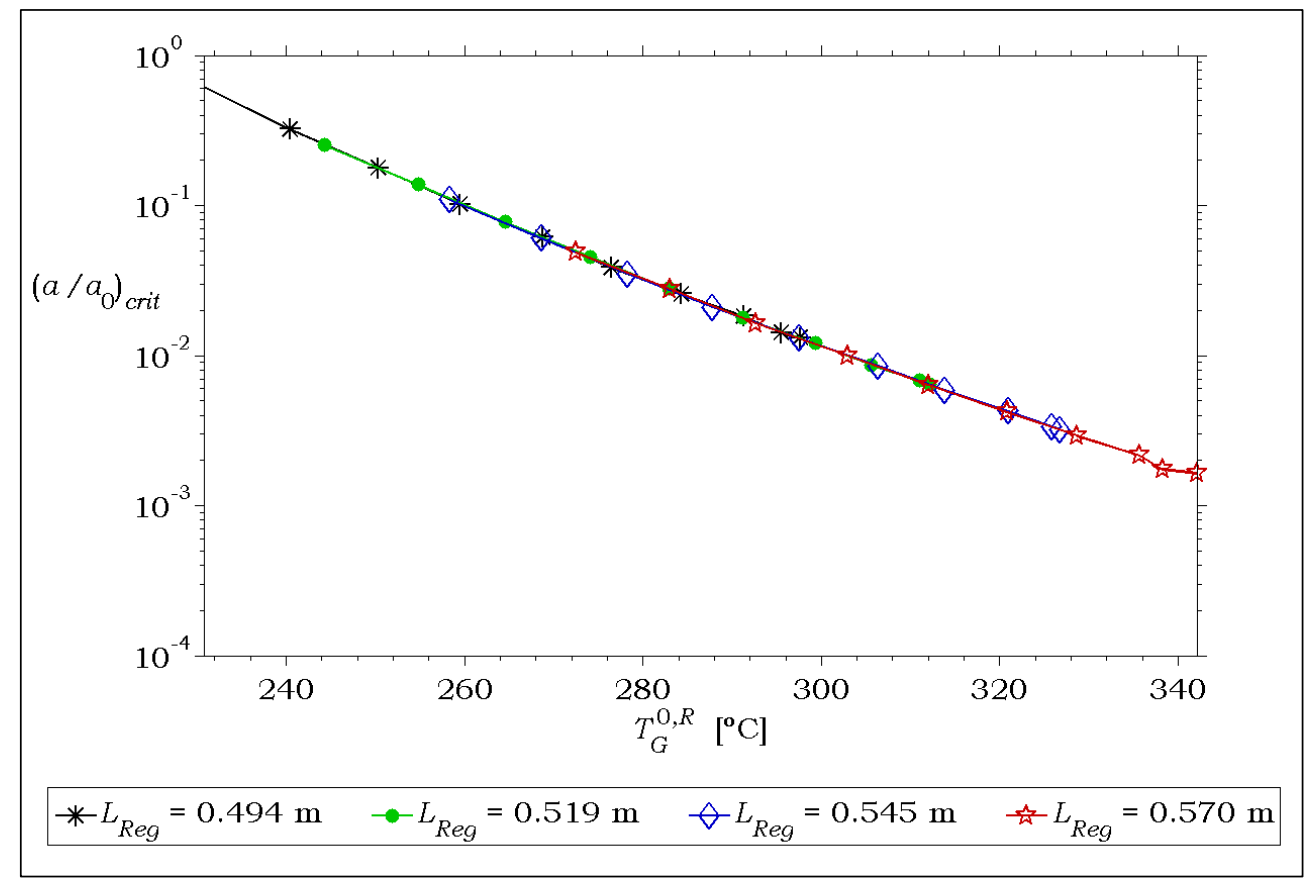

Figura 6.4: actividad crítica del catalizador vs. la temperatura de alimentación al reactor $\left(f_{L}=1 \%\right)$. 


\subsection{Conclusiones y comentarios finales}

En primer lugar, se discutieron brevemente las diferencias entre este esquema y los de inversión de flujo, centrado en el rol que posee el catalizador en uno y otro caso. Debido a que en esta variante el catalizador forma parte únicamente del reactor catalítico y que, en el css, el mismo opera en forma estacionaria, se concluyó que el comportamiento de este sistema frente a variaciones en algunos parámetros (por ejemplo, en la cantidad total de VOCs), no diferirá del dado para un esquema autotérmico de tipo "convencional".

Considerando lo anterior, en este capítulo solo se analizó la respuesta del sistema frente a variaciones en el tiempo de ciclo, la fracción de limpieza y la caída en la actividad del catalizador.

A través del estudio sobre la fracción de limpieza se pudo concluir que la inclusión de una pequeña región de la sección total (menor al 3\%), utilizada para el barrido de los VOCs alojados en los canales previos a la inversión (pasaje de la región de enfriamiento a la región de calentamiento), no perjudica la operación desde el punto de vista de la temperatura de entrada. Por el contrario, permite disminuir los niveles de emisión para todos los tiempos de ciclo considerados, respecto de los valores observados sin utilizar una fracción destinada a limpieza $\left(f_{L}=0\right)$. En adición, se corroboró que el caudal necesario para la limpieza no depende de $f_{L}$, dentro del intervalo considerado, y que el valor óptimo de tal fracción corresponde a aquel que permite mantener la velocidad másica entre cada una de las regiones en un mismo valor. Esto corresponde a una fracción de limpieza que oscila entre 0.06 y $0.6 \%$. No obstante, se recomienda adoptar un valor de $1 \%$, debido a que el mismo no genera diferencias apreciables y que el caudal de limpieza depende no solo del tiempo de ciclo, sino también de la longitud del regenerador (ver Ec. 6.1). En todos los casos, se puede considerar que estos valores de $f_{L}$ no traerán aparejados un cambio significativo en la pérdida de carga, respecto del valor que la misma presentará cuando no existe zona de limpieza.

Por otro lado, se analizó la respuesta del sistema frente a una caída en la actividad del catalizador, observando que este sistema resulta más sensible a la misma, en términos de la emisión de VOCs (comparar las Figuras 5.13 y 6.2), pero que la temperatura de entrada al reactor (y por ende la máxima), no 
se ve prácticamente afectada. Este análisis se complementó con una búsqueda de la actividad crítica (valor más pequeño de actividad que permite mantener un estado encendido para un tiempo de ciclo y longitud definidos) para diferentes tiempos de ciclo y longitudes del regenerador. Como se anticipara, se pudo corroborar a partir de ello que la variación en el valor de actividad crítica con cualquiera de los dos parámetros anteriores se debe enteramente a la variación en la temperatura de alimentación al reactor. 


\section{Apéndice 6.1: Análisis cualitativo del esquema au- totérmico}

A través del Capítulo 5 y del presente, se ha visto que tanto el sistema de inversión de flujo como el formado por el conjunto regenerador-reactor poseen una característica común. En el estado estacionario cíclico (css) apagado, la emisión de VOCs corresponde prácticamente al contenido total de la alimentación (es decir, la conversión de VOCs es nula). Por el contrario, el css encendido presenta niveles de emisión muy por debajo de dicho valor (normalmente, del orden de las decenas de ppm como máximo).

Este comportamiento tan extremo en términos de la conversión puede entenderse mediante una analogía con un sistema autotérmico convencional hipotético, conformado por un intercambiador en contra-corriente y el propio reactor adiabático analizado aquí. Recordando que el comportamiento térmico del regenerador se asemeja al de un intercambiador como el mencionado, el planteo de tal analogía para el caso del esquema regenerador-reactor es directa, razón por la cual se incluye este análisis en el presente capítulo. No obstante, si bien puede requerir un nivel de abstracción mayor, las conclusiones serán generales para los diferentes tipos de esquemas en estudio.

Para efectuar la analogía se considera un único VOC (el acetato de etilo, por ser el más representativo), ajustando su concentración de forma tal que el salto térmico adiabático sea de $40^{\circ} \mathrm{C}(571 \mathrm{ppm})$. En cuanto a las dimensiones de cada equipo, se toman como base los mismos valores de longitud y diámetro considerados previamente (ver Tabla 6.1).

A partir de la Ec. (A3.3.7) (ver Apéndice 3.3) la temperatura de alimentación al reactor, $T_{G}^{0, R}$, se puede escribir como:

$$
T_{G}^{\mathrm{O}, R}=T_{G}^{\mathrm{O}}+\psi \Delta T^{R}
$$

donde $\Delta T^{R}$ representa el salto térmico en el reactor, $T_{G}^{0}$ la temperatura de alimentación al sistema y $\psi$ la capacidad del intercambiador. Esta última se define como: 
$\psi=\frac{U A}{G_{m, t o t} c_{p G}}$

siendo $U$ el coeficiente global de transferencia de energia, $A$ el área de intercambio, $G_{m, t o t}$ el caudal másico total de la corriente a tratar y $c_{p G}$ el calor específico del gas.

Teniendo en cuenta la Ec. (A3.3.9), la capacidad del intercambiador (Ec. A6.1.2) puede reescribirse en términos de las variables en el regenerador:

$$
\psi=\psi\left(T_{G}^{0, R}\right)=\frac{1}{G_{m, t o t} c_{p G}} \frac{h_{m o d}\left(\left(T_{G}^{0}+T_{G}^{0, R}\right) / 2\right)}{2} \frac{a_{v} V_{R e g}}{2}
$$

siendo $h_{\text {mod }}$ el coeficiente de transferencia de energía modificado (ver Ec. 3.10), $V_{R e g}$ el volumen total del regenerador y $a_{v}$ el área total de transferencia por unidad de volumen total.

Por otro lado, los balances de materia y energía para el reactor quedan representados por el conjunto de ecuaciones diferenciales algebraicas definido en el Capítulo 3 (Ecs. 3.25 a 3.28), que para el caso de una única reacción se reducen a:

$$
\begin{aligned}
& G c_{p G} \frac{d T_{G}}{d z}=-a_{v} h_{\text {mod }}\left(T_{G}-T_{S}\right) \\
& \frac{G}{M_{\text {aire }}} \frac{d y_{G}}{d z}=-a_{v} k_{m} C_{T}\left(y_{G}-y_{S}\right) \\
& h_{\text {mod }}\left(T_{G}-T_{S}\right)+\ell\left(-\Delta H^{\#}\right) r^{e f}=0 \\
& k_{m} C_{T}\left(y_{G}-y_{S}\right)-\ell r^{e f}=0
\end{aligned}
$$

donde $T_{S}$ es la temperatura de la fase sólida en el reactor, $\ell$ la longitud característica del catalizador, $C_{T}$ la concentración molar total en la fase gaseosa y 
$M_{\text {aire }}$ el peso molecular del aire. $\Delta H^{\#}, r^{e f}$ y $k_{m}$ son la entalpía de combustión, la velocidad de consumo efectiva y el coeficiente de transferencia de materia del acetato de etilo, respectivamente. Finalmente, $y_{G}$ e $y_{S}$ son la fracción molar del acetato de etilo en la fase gaseosa y en la superficie del sólido, respectivamente.

Una forma usual de visualizar los posibles estados estacionarios asociados a las Ecs. (A6.1.1) a (A6.1.7), es tomando la temperatura de alimentación al reactor como un parámetro. Para ello, eliminando los términos de transferencia y velocidad de reacción entre las ecuaciones (A6.1.4) a (A6.1.7), se puede reducir el balance de energía en la fase gaseosa a:

$$
T_{G}(z)-T_{G}^{0, R}=\frac{\left(-\Delta H^{\#}\right) y_{G}^{0}}{M_{\text {aire }} c_{p G}}\left(1-y_{G} / y_{G}^{0}\right)=\Delta T_{a d} x(z)
$$

donde $x=1-y_{G} / y_{G}^{0}$ es la conversión de acetato de etilo (se desprecia el cambio en el caudal molar total) e $y_{G}^{0}$ es la fracción molar de acetato de etilo en la corriente a tratar. Evaluado la Ec. (A6.1.8) en la salida del reactor (define el salto térmico en el reactor) y reemplazando en la Ec. (A6.1.1), se tiene ${ }^{9}$ :

$$
x=\frac{T_{G}^{0, R}-T_{G}^{0}}{\psi\left(T_{G}^{0, R}\right) \Delta T_{a d}}
$$

Por otro lado, incorporando la expresión de $T_{G}$ (Ec. A6.1.8) en la Ec. (A6.1.6) y reacomodando, los restantes balances se pueden re-escribir como:

$$
\frac{d x}{d V}=\frac{M_{\text {aire }} a_{v} k_{m} C_{T}}{G_{m, t o t}}\left[(1-x)-\frac{y_{S}}{y_{G}^{0}}\right]
$$

\footnotetext{
9 Notar que, debido a la variación del coeficiente de transferencia de energía con la temperatura, la Ec. (A6.1.9) no resulta lineal con $T_{G}^{0, R}$.
} 


$$
\begin{aligned}
& h_{\text {mod }}\left[\left(T_{G}^{0, R}+\Delta T_{a d} x\right)-T_{S}\right] /\left(-\Delta H^{*}\right)+\ell r^{e f}=0 \\
& k_{m} C_{T}\left[y_{G}^{0}(1-x)-y_{S}\right]-\ell r^{e f}=0
\end{aligned}
$$

Habiendo definido la temperatura de alimentación al reactor, se puede resolver el conjunto de ecuaciones diferenciales algebraicas definidas por las Ecs. (A6.1.10) a (A6.1.12), obteniendo $T_{S}, y_{S}$ y la conversión $x$. Luego, graficando por un lado la curva que define $x$ en función de $T_{G}^{O, R}$ (Ec. A6.1.9), y por otro lado el valor de $x$ calculado para cada valor de $T_{G}^{0, R}$ a partir de las Ecs. (A6.1.10) a (A6.1.12), las intersecciones de ambas representaciones definen los estados estacionarios posibles. Esto se muestra en la Figura A6.1.1 para el caso "base" definido al inicio de la presente sección (la línea de trazos corresponde a la Ec. A6.1.9 y la llena a las Ecs. A6.1.10 a A6.1.12). Allí se ve claramente la presencia de dos estados estacionarios de tipo "estables" (uno de ellos con conversión prácticamente nula y otro con una conversión completa) y otro de tipo "inestable" (presumiblemente presente en los esquemas analizados en el Capítulo 5 y en el presente, pero imposible de visualizar debido a la naturaleza de la resolución).

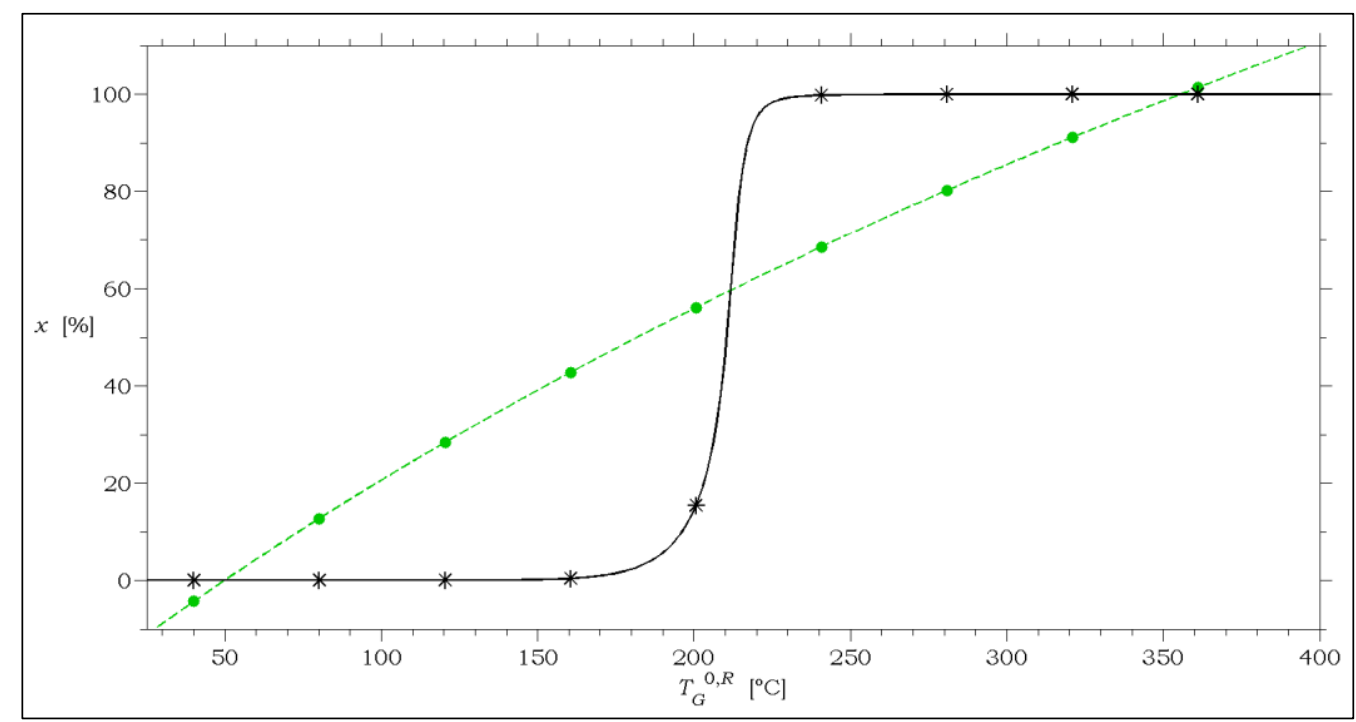

Figura A6.1.1: solución al conjunto de Ecuaciones (A6.1.9) a (A6.1.12) para el caso base.

Por otro lado, en la Figura A6.1.2 se muestran las curvas obtenidas para valores decrecientes de la capacidad de intercambio, lograda a partir de una 
reducción del volumen (que resultaría equivalente a aumentar el tiempo de ciclo del regenerador). Los valores informados en el gráfico están evaluados a la temperatura correspondiente a la solución de estado estacionario encendida.

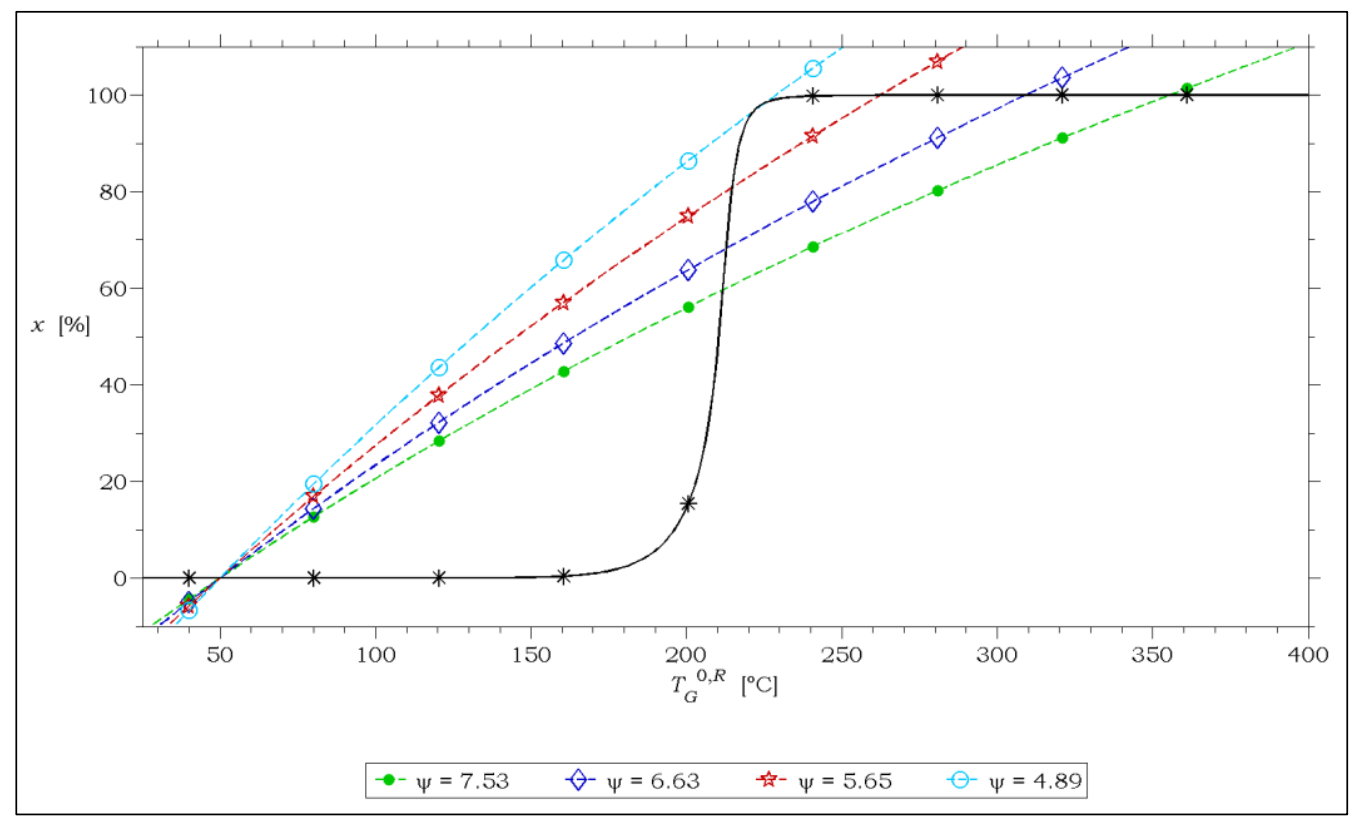

Figura A6.1.2: conjunto de soluciones obtenidas al disminuir la capacidad de intercambio.

En la Figura A6.1.2 se puede ver que la conversión permanece prácticamente inalterada al variar $\psi$, excepto en las cercanías con la transición entre el estado encendido y apagado. De hecho, el punto en donde una posterior disminución de dicho parámetro conducirá a un único estado estacionario apagado, corresponde a $x \approx 97.6 \%(13.7 \mathrm{ppm})$.

En forma similar, en la Figura A6.1.3 se varia la actividad del catalizador en lugar de variar $\psi$ (ver Sección 6.2 para los resultados en el sistema regenerador-reactor con el modelo completo). En este caso la curva definida por la Ec. (A6.1.9) no se ve alterada y en su lugar se modifica la curva de conversión obtenida de las Ecs. (A6.1.10) a (A6.1.12). Para tales condiciones, la conversión en el punto previo a la desaparición del estado estacionario encendido corresponde a un $97.7 \%$ aproximadamente (13.1 ppm), observando que el comportamiento de la conversión en la transición del estado encendido al apagado no difiere del anterior.

En adición, la formulación presentada permite concluir que, cuanto más elevada sea la energía de activación de la reacción involucrada, la conversión 
predicha por las Ecs. (A6.1.10) a (A6.1.12) tenderá a comportarse como un "escalón". En tal caso, los estados estacionarios estables solo podrian presentar una conversión nula o total.

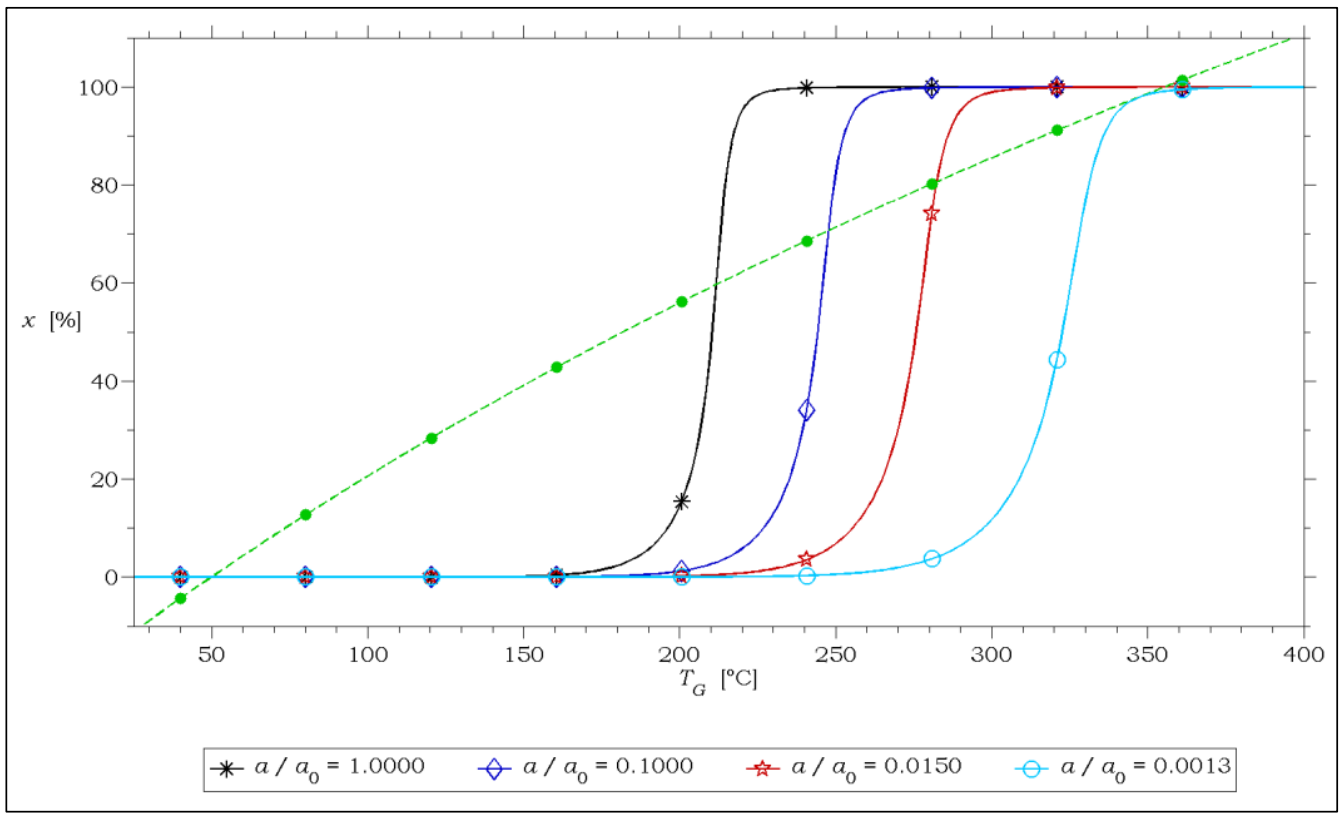

Figura A6.1.3: conjunto de soluciones obtenidas al disminuir la actividad del catalizador.

En resumen, mediante este análisis pueden entenderse los valores extremos de conversión de VOCs en este tipo de sistemas. Tal comportamiento está íntimamente asociado a la presencia de reacciones con elevadas energías de activación (para el acetato de etilo el valor de este parámetro es de $1.4810^{5} \mathrm{~J} / \mathrm{mol}$-aproximadamente $\left.35000 \mathrm{cal} / \mathrm{mol}-\right)$ y a una elevada diferencia entre la temperatura de alimentación al sistema frente a la temperatura de reacción (elevadas capacidades de intercambio). 


\section{Capítulo 07}

\section{- Diseño de los sistemas de inversión de flujo}

- Diseño del esquema convencional

- Diseño del esquema rotativo 

En los capítulos previos se han definido los modelos y métodos numéricos para la simulación de cada sistema y se ha estudiado la respuesta de cada uno de ellos frente a variaciones en los principales parámetros. El objetivo de tal análisis fue lograr un mejor conocimiento de los sistemas bajo estudio, entendiendo que ello es primordial para establecer criterios de diseño que resulten adecuados.

Particularmente, se ha podido verificar en los Capitulos 5 y 6 que los tres esquemas propuestos se caracterizan por solo dos tipos posibles de estados estacionarios cíclicos estables. El primero corresponde a un estado apagado con conversión prácticamente nula. Por el contrario, el segundo representa un estado encendido con una muy elevada conversión (usualmente mayor al 98\%), o en términos de la emisión de VOCs, con una emisión residual no mayor a los 10-20 ppm. Más aún, siempre que todos los parámetros operativos estén alejados de los valores críticos ${ }^{10}$, la emisión residual de VOCs normalmente oscila en valores menores a los $2 \mathrm{ppm}$ (ver Capítulos 5 y 6). Consecuentemente, es esperable que si se ajustasen las dimensiones de alguno de los sistemas (o una variable operativa si correspondiera), de forma tal que cumpliera estrictamente con la cota de emisión de 9 ppm mencionada en el Capítulo 2, el diseño alcanzado (o la condición operativa seleccionada) fuese relativamente inestable. Esta situación definitivamente debe evitarse, por lo cual tendrá que ser considerada en los criterios de diseño.

Para cada uno de los esquemas presentados, se pretende alcanzar un diseño que permita tratar la corriente de aire contaminada con VOCs, detallada en el Capítulo 2, cuyas características se repiten en la Tabla 7.1. Como se mencionó en dicho capítulo, usualmente las condiciones de la misma podrán fluctuar, especialmente el contenido total de VOCs. Por esta razón, un adecuado criterio de diseño, para cualquiera de los esquemas, no solo deberá considerar la concentración nominal de VOCs en la corriente, sino un rango posible convenientemente elegido. En base a lo discutido en los capítulos anteriores, se puede concluir que el diseño de cualquiera de los sistemas analizados en el presente estudio se verá perjudicado cuanto más bajo sea el contenido de VOCs. Por esta razón, se ha considerado una concentración de

10 Entendiendo como tal el valor umbral de un parámetro dado, a partir del cual un aumento o disminución del mismo, según corresponda, conduce a la extinción de las reacciones. 
acetato de etilo y etanol mínima, tal como fuera mencionado en el Capítulo 2. Dicho valor se indica también en la Tabla 7.1.

Tabla 7.1: características de la corriente a tratar.

\begin{tabular}{|c|c|c|c|c|c|c|}
\hline $\begin{array}{c}\mathbf{G}_{m, t o t} \\
{[\mathrm{~kg} / \mathrm{s}]}\end{array}$ & $\begin{array}{c}\boldsymbol{P}^{0} \\
\text { [bar] }\end{array}$ & $\begin{array}{c}\mathbf{T}_{G}^{0} \\
{\left[{ }^{\circ} \mathbf{C}\right]}\end{array}$ & (m) & na) & (no & $\begin{array}{l}\text { nal) } \\
\text { a] }\end{array}$ \\
\hline \multirow{2}{*}{12.7} & \multirow{2}{*}{1.01} & \multirow{2}{*}{50} & $\mathrm{AE}$ & $\mathrm{Et}$ & $\mathrm{AE}$ & Et \\
\hline & & & 307 & 125 & 615 & 250 \\
\hline
\end{tabular}

Como fuera señalado en el Capítulo 5, el esquema convencional y el rotativo son, desde el punto de vista del comportamiento, equivalentes. La principal diferencia se da en la posibilidad de operar el segundo a muy bajos tiempos de ciclo, gracias a la incorporación de la región de limpieza que permite eliminar el problema de la emisión de VOCs sin tratar, cuando se invierte el sentido de circulación. En adición, cabe recordar que el tiempo de ciclo mínimo en el sistema convencional está determinado por la capacidad de respuesta de las válvulas y su vida útil, mientras que en el rotativo queda definido por la máxima velocidad de rotación admisible.

Las diferencias señaladas en el párrafo anterior deberán ser tenidas en cuenta en los criterios de diseño, los cuales no serán estrictamente iguales. Por esta razón, se abordará todo lo concerniente al dimensionamiento (criterios, búsqueda y resultados) por separado para cada uno de los esquemas.

\subsection{Diseño del esquema convencional}

Uno de los primeros pasos a seguir para establecer los criterios y estrategia de diseño es identificar los grados de libertad y restricciones de cada sistema.

Asumiendo que se conoce la configuración de la celda, un análisis del esquema de inversión de flujo convencional y su descripción (ver Capítulo 3), permite identificar cuatro grados de libertad para el mismo. Estos son: 
* la fracción de inerte,

* el volumen total del equipo,

* el diámetro del equipo,

* el tiempo de ciclo,

De los grados de libertad identificados, los tres primeros corresponden a variables estrictamente de diseño (volumen, diámetro y fracción de inerte). Por el contrario, el tiempo de ciclo representa una variable operativa y por lo tanto merece una consideración adicional. El valor de este parámetro podrá ser diferente para las condiciones nominales o las de mínimo contenido de VOCs, lo que permite abarcar el rango propuesto, sujeto a una serie de condiciones determinadas.

En lo que respecta a las restricciones, la única establecida a priori en el sistema corresponde a la cota de emisión global de 9 ppm (ver Sección 2.3.1). No obstante, como se discutió en la introducción del presente capítulo, tal valor de emisión se obtiene típicamente para condiciones cercanas al límite entre los estados apagado y encendido. Por esta razón, se exige alternativamente que la emisión de VOCs sea de $\mathbf{1}$ ppm para la condición de operación con el mínimo contenido de VOCs (situación más desfavorable).

En adición, si se pretende utilizar un soplador convencional para impulsar la corriente a tratar será necesario limitar la pérdida de carga en el sistema. Para ello, se exige que la pérdida de carga no supere los $\mathbf{5 0}$ mbar en ningún caso (es decir $|\Delta P| \leq 50$ mbar).

Otro aspecto a considerar es que la temperatura máxima en el lecho no deberá superar un cierto nivel a fin de evitar la temprana desactivación del catalizador empleado. No disponiendo de datos sobre la estabilidad del catalizador frente a la temperatura, se adoptó un valor de temperatura máxima admisible en el lecho de $400{ }^{\circ} \mathbf{C}$ (es decir, $T_{S, \max } \leq 400^{\circ} \mathrm{C}$ ). Sin embargo, se asume que dicho valor es conservativamente bajo. De esta manera, a lo largo del período de vida útil del catalizador se dispondría de cierto margen para elevar la temperatura de operación, a fin de compensar los efectos de desactivación del mismo de ser necesario. 
La cantidad y tipo de restricciones enunciadas hasta aquí no permiten anular completamente los grados de libertad señalados y, consecuentemente, no podrá establecerse un único diseño. Para solventar esto se deben definir restricciones adicionales, fijar en forma directa los valores de algunos de los grados de libertad enunciados o definir una función objetivo y seleccionar el diseño que minimice/maximice la misma. Esta última opción se descartó debido a que la búsqueda de un diseño óptimo cae fuera de los alcances propuestos para el presente estudio, quedando solo las dos primeras alternativas.

Teniendo en cuenta que el material catalítico será presumiblemente más costoso que el inerte, se fijó la fracción de inerte en el equipo, $f_{I}$, en un $\mathbf{4 0 \%}$ (valor que puede considerarse razonablemente elevado).

Por otro lado, de acuerdo a Barresi y col. (2007), las válvulas utilizadas en los sistemas de inversión de flujo soportan en promedio unos 500000 movimientos o 250000 ciclos. Por lo tanto, será deseable establecer un límite inferior para el tiempo de ciclo. Definiendo la mínima vida útil tolerable de la válvula, se puede determinar el tiempo de ciclo minimo para el sistema. De esta manera, si se pretende que la vida útil de la válvula sea de al menos 2 años, el tiempo de ciclo no podrá ser menor a $240 \mathrm{~s}$ (es decir, $t_{\text {ciclo }} \geq 240 \mathrm{~s}$ ).

Para completar la propuesta de diseño es conveniente considerar dos aspectos adicionales. Por un lado, el mínimo volumen necesario será mayor cuanto menor sea el contenido de VOCs a tratar y más elevado sea el tiempo de ciclo. Luego, el volumen total del equipo, $V_{e q}$, se determinará para la condición de operación con el mínimo contenido de VOCs y utilizando el menor tiempo de ciclo posible (240 s para este caso). Por otro lado, será deseable que tanto el tiempo de ciclo para la condición de operación con el contenido de VOCs nominal, así como el diámetro del equipo, $D_{e q}$, sean bajos (el primero para que el estado de operación se encuentre alejado del límite con el css apagado y el segundo para facilitar una correcta distribución del flujo). Para ello, teniendo en cuenta que tanto la mayor pérdida de carga como la máxima elevación de temperatura en el lecho se encontrarán para la condición de operación con el contenido de VOCs nominal, se exige que en tal condición se verifique simul- 
táneamente ${ }^{11} T_{S, \max }=400{ }^{\circ} \mathrm{C}$ y $|\Delta P|=50$ mbar. Cabe aclarar que podría resul$\operatorname{tar} T_{S, \max }<400^{\circ} \mathrm{C}$ en la condición nominal, aún para el mínimo tiempo de ciclo. En tal caso, se adoptará también $t_{\text {ciclo }}=240 \mathrm{~s}$ para la misma. Así, sin explicitar la secuencia iterativa, los criterios enunciados se pueden resumir según:

* Fijar la fracción de inerte en $40 \%$.

* Para el contenido de VOCs mínimo:

- fijar la emisión global en 1 ppm y el tiempo de ciclo en su menor valor (240 s). Determinar el volumen del equipo.

* Para el contenido de VOCs nominal:

- si para el menor tiempo de ciclo, la temperatura máxima en el lecho resultase menor a $400{ }^{\circ} \mathrm{C}$, fijar el tiempo de ciclo en su valor mínimo (240 s) y determinar el diámetro que permita satisfacer $|\Delta P|=50$ mbar;

\ caso contrario, determinar el diámetro y el tiempo de ciclo que permitan satisfacer en simultáneo $|\Delta P|=50$ mbar y $T_{S, \max }=400{ }^{\circ} \mathrm{C}$.

Finalmente, se propone el estudio de cinco configuraciones de celda, presentadas en la Tabla 7.2, donde se numeró cada una para facilitar su identificación. Las tres primeras corresponden a formas estándar comercializadas por la empresa Corning (Boger y col., 2004). Las 4 y 5 son alternativas a las 2 y 3 , respectivamente, variando el espesor de tabique de forma tal que la porosidad sea la misma que para la configuración 1, aspecto que se discutirá con mayor detalle en la Sección 7.1.1.2. El significado de los parámetros indicados en dicha tabla es:

$$
\begin{aligned}
& N_{C} \quad \text { número de celdas (canales) por unidad de superficie transversal (el } \\
& \text { número entre paréntesis indica su valor en canales por pulgada } \\
& \text { cuadrada). }
\end{aligned}
$$

\footnotetext{
11 Nótese que las restricciones que permitirán ajustar el volumen y diámetro del equipo quedan definidas en condiciones diferentes, lo cual conduce a un cálculo de tipo iterativo.
} 
$\delta_{T} \quad$ espesor total de tabique (el número entre paréntesis indica su valor en milésimas de pulgada).

$\varepsilon_{L} \quad$ porosidad del lecho (fracción de sección transversal disponible para el flujo).

$a_{v} \quad$ área de transferencia por unidad de volumen total.

$d_{h}=4 \varepsilon_{L} / a_{v}$. Diámetro hidráulico. Notar que, para el caso considerado en el presente estudio (radio de curvatura nulo en los rincones del canal), resulta exactamente igual al lado del canal $\left(L_{C}-\delta_{T}\right)$, donde $L_{C}$ es la longitud de celda (ver Apéndice 3.2).

$R_{f} \quad=a_{v}^{2} / \varepsilon_{L}^{3}$. Factor de pérdida de carga. Esta agrupación condensa los efectos geométricos sobre la pérdida de carga, lo cual se puede ver reordenando la Ec. (3.19) según:

$\left(\rho_{G}|\Delta P|\right) /\left(L_{e q} \mu_{G} G\right)=1 / 8(f \operatorname{Re}) R_{f}$. Luego, suponiendo que $G$ está definido, será deseable un valor pequeño de $R_{f}$ para que la pérdida de carga resulte baja.

$K_{V} \quad=a_{v}^{2} / \varepsilon_{L}$. Factor de transferencia de materia o energía por unidad de volumen total. Suponiendo que los efectos de entrada son despreciables $^{12}$, esta agrupación condensa los efectos geométricos del canal sobre la capacidad de transferencia de materia $\left(a_{v} k_{m, j}\right)$ o de energía $\left(a_{v} h\right)$. Esto puede verse explicitando sendos productos de las Ecs. (A3.1.13) y (A3.1.16) (con los valores asintóticos del Sh y del $N u, S h_{\infty}$ y $N u_{\infty}$, respectivamente) según: $a_{v} k_{m, j}=1 / 4 D_{m, j} S h_{\infty} K_{V}$ y $a_{v} h=1 / 4 \lambda_{G} N u_{\infty} K_{V}$. En estas condiciones será deseable que la configuración del monolito posea elevados valores de $K_{V}$.

$H_{C} \quad=K_{V} /\left(1-\varepsilon_{L}\right)$. Factor de calentamiento del sólido (o coeficiente de encendido). Este parámetro está estrechamente vinculado con la

12 En el caso de la transferencia de energía, se debe asumir también que el efecto de la conducción térmica axial del sólido es despreciable. 
velocidad a la cual se calienta (o enfría) el sólido. Contrariamente a otros procesos, para la presente aplicación este parámetro deberá ser pequeño, para favorecer la estabilidad del sistema.

MIF $=\left(\delta_{T} / L_{C}\right)^{2} /\left[1-\left(\delta_{T} / L_{C}\right)\right]$. Factor de resistencia al aplastamiento en dirección diagonal. Directamente relacionado con la resistencia estructural del monolito.

Tabla 7.2: configuraciones de celda y propiedades de las mismas.

\begin{tabular}{|c|c|c|c|c|c|c|c|c|c|}
\hline $\mathbf{N}^{\circ}$ & $\begin{array}{c}10^{-5} N_{C} \\
{\left[\mathrm{~m}^{-2}\right]}\end{array}$ & $\begin{array}{c}10^{4} \delta_{T} \\
{[\mathrm{~m}]}\end{array}$ & $\varepsilon_{L}$ & $\begin{array}{c}a_{v} \\
{\left[\mathbf{m}^{-1}\right]}\end{array}$ & $\begin{array}{c}10^{3} d_{h} \\
{[\mathrm{~m}]}\end{array}$ & $\begin{array}{c}10^{-7} R_{f} \\
{\left[\mathrm{~m}^{-2}\right]}\end{array}$ & $\begin{array}{c}10^{-6} K_{V} \\
{\left[\mathrm{~m}^{-2}\right]}\end{array}$ & $\begin{array}{c}10^{-7} H_{C} \\
{\left[\mathrm{~m}^{-2}\right]}\end{array}$ & $10^{2} \mathrm{MIF}$ \\
\hline 1 & $\begin{array}{c}3.1 \\
(200)\end{array}$ & $\begin{array}{c}3.18 \\
(12.5)\end{array}$ & 0.678 & 1833 & 1.48 & 1.08 & 4.96 & 1.54 & 3.80 \\
\hline 2 & $\begin{array}{c}6.2 \\
(400)\end{array}$ & $\begin{array}{l}1.91 \\
(7.5)\end{array}$ & 0.722 & 2677 & 1.08 & 1.90 & 9.92 & 3.57 & 2.65 \\
\hline 3 & $\begin{array}{c}9.3 \\
(600)\end{array}$ & $\begin{array}{c}1.02 \\
(4)\end{array}$ & 0.814 & 3480 & 0.94 & 2.25 & 14.9 & 7.98 & 1.06 \\
\hline 4 & $\begin{array}{c}6.2 \\
(400)\end{array}$ & $\begin{array}{l}2.25 \\
(8.8)\end{array}$ & 0.678 & 2593 & 1.05 & 2.16 & 9.92 & 3.08 & 3.80 \\
\hline 5 & $\begin{array}{c}9.3 \\
(600)\end{array}$ & $\begin{array}{l}1.83 \\
(7.2)\end{array}$ & 0.678 & 3176 & 0.85 & 3.24 & 14.9 & 4.62 & 3.80 \\
\hline
\end{tabular}

\subsubsection{Determinación de los rangos de búsqueda}

Lógicamente, como en cualquier proceso de búsqueda (ya sea de raíces de un sistema de ecuaciones y/o de valores óptimos), es deseable, eventualmente necesario, disponer de valores iniciales para comenzar el mismo o mejor aún, de un rango de valores en donde se encuentre el punto buscado.

Existen algunos trabajos en bibliografia que abordan la problemática del dimensionamiento del sistema desde un punto de vista simplificado. Destacan particularmente tres métodos, los cuales han sido resumidos y comparados por Marín y col. (2009). Todos ellos se basan en dos elementos básicos: la aproximación a través del reactor en contra-corriente y el movimiento del frente de onda (ver Capítulo 5). El más claro desde un punto de vista conceptual 
es quizás el método propuesto por Cittadini y col. (2001), el cual, de acuerdo con Marín y col. (2009), resulta más adecuado para un diseño preliminar, estimando la longitud del reactor por exceso en todos los casos. Si bien dicho método está basado en un sistema donde solo existe una única reacción con cinética lineal, los conceptos mencionados no dependen de ello y, por lo tanto, serán utilizados para establecer los rangos de búsqueda.

Cabe aclarar que el objetivo en los trabajos mencionados es establecer un diseño directamente basado en las simplificaciones allí propuestas. Sin embargo, aquí se propone un camino alternativo: basándose en la aproximación del reactor en contra-corriente, es posible establecer una cota inferior para las dimensiones del sistema. Luego, considerando el movimiento del frente de onda, se puede estimar una cota superior ${ }^{13}$. Para ello, debe tenerse en cuenta que la operación a tiempos de ciclo bajos es más eficiente, en términos de la capacidad para retener la energía liberada por la reacción. Luego, las dimensiones requeridas por el reactor en contra-corriente representarán una cota inferior de volumen y diámetro, $V_{e q, \min } \mathrm{y} D_{e q, \min }$, respectivamente. Por otro lado, teniendo en cuenta el movimiento del frente de onda, resulta inmediato que el sistema de inversión de flujo deberá disponer de un volumen total mayor que el mínimo, de modo tal que permita el desplazamiento de dicho frente durante el tiempo que durase el semiciclo. Por lo tanto, definido un tiempo de ciclo, el conocimiento de una cota máxima para la velocidad a la que se desplazaría el frente de onda, permitirá estimar el volumen necesario para tal desplazamiento y, en consecuencia, se podrá disponer de una cota superior de volumen, $V_{e q, \max }$, y de diámetro, $D_{e q, \max }$.

\subsubsection{Cota inferior de volumen y diámetro del equipo}

Recordando que el modelo del reactor en contra-corriente está representado por un conjunto de ecuaciones diferenciales algebraicas (Sección 3.5), la búsqueda de las cotas inferiores de volumen y diámetro mediante este modelo será iterativa, debiendo resolver dicho conjunto de ecuaciones en cada paso de la iteración. Por esta razón, se expone en esta sección un cálculo aproximado

\footnotetext{
13 Debe tenerse presente que este procedimiento resultará adecuado en la medida que los tiempos de ciclo deseados sean relativamente bajos. De lo contrario, si bien aún serviría como un marco de referencia, el rango obtenido sería muy amplio.
} 
que permite obtener una primera estimación de dichas cotas. Cabe resaltar que, aun representando un buen punto de partida para tal iteración, los valores obtenidos con esta formulación no son adecuados como cotas en forma directa, pues resultan menores que los valores obtenidos con el modelo detallado del reactor en contra-corriente, perjudicando la estimación de la cota superior (ver Sección 7.1.1.2).

Suponiendo que todo el lecho es catalítico y utilizando las mismas simplificaciones dadas en la Sección 5.1 en lo que respecta al comportamiento térmico, el volumen del reactor en contra-corriente se puede descomponer en dos contribuciones. Una de ellas corresponde a las regiones extremas del reactor en donde la corriente eleva su temperatura hasta el valor de $T_{i g n} \approx 220{ }^{\circ} \mathrm{C}$, con un volumen que se identificará como $V_{p h}$. La otra corresponde al volumen necesario para que la reacción proceda hasta el nivel requerido, el cual se identificará como $V_{R}^{*}$.

En primer lugar, se puede evaluar el volumen $V_{p h}$ a partir de la Ec. (5.3). Considerando las propiedades constantes (evaluadas a la temperatura media entre $T_{G}^{0}$ y $T_{i g n}$ ) y utilizando el valor asintótico del coeficiente de transferencia de energía (indicado como $h_{\infty}$ ), sin la modificación para contabilizar el efecto de la conducción axial térmica ${ }^{14}$, se tiene:

$$
V_{p h}=\frac{G_{m, t o t} c_{p G}}{a_{v} h_{\infty}\left(\Delta T_{a d} / 2\right)}\left(T_{i g n}-T_{G}^{0}\right)=\frac{4 G_{m, t o t} c_{p G}}{\lambda_{G} N u_{\infty}} \frac{\left(T_{i g n}-T_{G}^{0}\right)}{\left(\Delta T_{a d} / 2\right)} \frac{1}{K_{V}}
$$

donde, de acuerdo con los criterios dados en la Sección 7.1, el valor de $\Delta T_{a d}$ debe corresponder a la condición de mínimo contenido de VOCs. Adicionalmente, se ha explicitado la dependencia con el factor geométrico $K_{V}$, discutido en la Sección 7.1.

Por otro lado, para evaluar el volumen $V_{R}^{*}$ se supone que toda la región donde hay reacción química permanece a una temperatura uniforme, e igual a

\footnotetext{
14 Ambas simplificaciones sobre el coeficiente de transferencia de energía se adoptan para eli-
} minar la dependencia del mismo con el diámetro del equipo (presente a través de la velocidad). 
$T_{i g n}+\Delta T_{a d}$ y que las reacciones están completamente controladas por la resistencia a la transferencia de materia en el canal. Bajo dicha condición, el acetaldehído no alcanzará a acumularse y por lo que solo se necesitan los balances de materia para los dos componentes restantes, que resultan independientes entre sí. Luego, a partir de las Ecs. (3.35) y (3.36), se puede obtener $V_{R}^{*}$ exigiendo que la cantidad remanente de VOCs a la salida sea de 1 ppm y resolviendo la Ec. (7.2), dada por:

$$
\begin{aligned}
\left\langle y_{\text {VOCs }}\right\rangle & =y_{1, G}^{0} \exp \left(-\frac{a_{v} \rho_{G} k_{m, 1, \infty}}{G_{m, \text { tot }}} V_{R}^{*}\right)+y_{2, G}^{0} \exp \left(-\frac{a_{v} \rho_{G} k_{m, 2, \infty}}{G_{m, t o t}} V_{R}^{*}\right) \\
& =y_{1, G}^{0} \exp \left(-\frac{\rho_{G} D_{m, 1} S h_{\infty}}{4 G_{m, t o t}} K_{V} V_{R}^{*}\right)+y_{2, G}^{0} \exp \left(-\frac{\rho_{G} D_{m, 2} S h_{\infty}}{4 G_{m, t o t}} K_{V} V_{R}^{*}\right)
\end{aligned}
$$

donde los valores de $y_{j, G}^{0}$ corresponden al mínimo contenido de VOCs. Además, al igual que antes, se ha utilizado el valor asintótico de los coeficientes de transferencia de materia, $k_{m, j, \infty}$, lo que permite explicitar la dependencia con el factor geométrico $K_{V}$.

Finalmente, la estimación del volumen total que servirá como punto de partida en la búsqueda de $V_{e q, \min }$ es

$$
V_{e q, \min }^{(0)}=2 V_{p h}+V_{R}^{*}
$$

donde el superíndice (0) indica el valor inicial para la iteración.

Las estimaciones dadas por las Ecs. (7.1) a (7.3) predicen una relación directamente proporcional entre el volumen mínimo y el factor $K_{V}$. Por lo tanto, puede esperarse que el volumen mínimo para las configuraciones con el mismo valor de $K_{V}$ (las 2 y 4 o las 3 y 5 ) sean muy similares.

Por otro lado, conocido el valor de $V_{e q, \min }^{(0)}$, estableciendo la pérdida de carga en el valor máximo admisible (50 mbar), se puede obtener también una prime- 
ra estimación para la cota inferior del diámetro del equipo, $D_{\text {eq, } m i n}^{(0)}$. Para ello, a partir de la Ec. (3.19), expresando $L_{e q, \min }^{(0)} G=16 V_{e q, \min }^{(0)} G_{m, t o t} /\left[\pi^{2}\left(D_{e q, \min }^{(0)}\right)^{4}\right]$ y considerando las propiedades constantes, se tiene:

$$
D_{e q, \min }^{(0)}=\left[\frac{2(f \mathrm{Re}) R_{f} G_{m, \text { tot }} V_{e q, \min }^{(0)}}{\pi^{2}} \frac{\left(\mu_{G} / \rho_{G}\right)_{T_{i g n}+\Delta T_{a d}}}{|\Delta P|}\right]^{1 / 4}
$$

Con las estimaciones iniciales dadas por las Ecs. (7.1) a (7.4), se comienza el proceso de búsqueda de las cotas inferiores de volumen y diámetro de equipo, dadas por el reactor en contra-corriente. Los valores obtenidos para cada configuración, para una fracción de inerte de $40 \%$ y un tiempo de ciclo de 240 s, se indican en la Tabla 7.3.

Tabla 7.3: cotas inferiores y superiores para el volumen y diámetro del equipo en el sistema de inversión de flujo convencional.

\begin{tabular}{ccc}
\hline $\mathbf{n}^{\circ}$ de configuración & $\begin{array}{c}\boldsymbol{V}_{\text {eq, } \min }-\boldsymbol{V}_{\text {eq, } \max } \\
{\left[\mathbf{m}^{\mathbf{3}]}\right.}\end{array}$ & $\begin{array}{c}\boldsymbol{D}_{\text {eq, } \boldsymbol{m i n}}-\boldsymbol{D}_{\text {eq, } \max } \\
{[\mathbf{m}]}\end{array}$ \\
\hline $\mathbf{1}$ & $3.98-5.81$ & $1.78-2.15$ \\
\hline $\mathbf{2}$ & $2.10-4.23$ & $1.76-2.29$ \\
\hline $\mathbf{3}$ & $1.42-4.63$ & $1.67-2.44$ \\
\hline $\mathbf{4}$ & $2.11-3.94$ & $1.82-2.32$ \\
\hline $\mathbf{5}$ & $1.44-3.28$ & $1.84-2.45$ \\
\hline
\end{tabular}

\subsubsection{Cota superior de volumen y diámetro del equipo}

El modelo del reactor en contra-corriente permite establecer la cota mínima de volumen, pero por su naturaleza no tiene en cuenta el movimiento del frente de onda en el sólido. Como fuera visto en el Capítulo 5, dicho frente se moverá a una determinada velocidad a lo largo del reactor. La propuesta básica para la determinación de la cota superior de volumen, $V_{\text {eq, max }}$, es que los 
perfiles no modifican su forma respecto a los del contra-corriente, conforme se aumenta en forma simultánea el tiempo de ciclo y el volumen del equipo. Esta concepción es la utilizada por Cittadini y col. (2001). Cabe recordar que, como señalan Marín y col. (2009), los valores calculados de esta manera resultan siempre conservativos, lo que justifica su elección como cota superior.

A partir de la simplificación propuesta para el cálculo de la cota superior, resta determinar la velocidad del frente de onda, $v_{F}$. En el sistema de inversión de flujo, este parámetro variará con el tiempo. No obstante, existen aproximaciones basadas en el concepto de un lecho de longitud infinita, asumiendo que dicho parámetro es independiente del tiempo (ver, por ejemplo, Matros $y$ Bunimovich, 1996; Thullie y Burghardt, 1995) dado por:

$$
\begin{aligned}
& \gamma=\frac{\left(\rho c_{p}\right)_{G}^{0}}{\varepsilon_{L}\left(\rho c_{p}\right)_{G}^{0}+\left(1-\varepsilon_{L}\right)\left(\rho c_{p}\right)_{S}} \approx \frac{\left(\rho c_{p}\right)_{G}^{0}}{\left(1-\varepsilon_{L}\right)\left(\rho c_{p}\right)_{S}} \\
& v_{F}=\frac{\gamma G_{m, t o t}}{\rho_{G}^{0} S_{t o t}}\left(\frac{1-\Delta T_{a d} / \Delta T_{R}}{1-\varepsilon_{L} \gamma \Delta T_{a d} / \Delta T_{R}}\right) \approx \frac{\gamma G_{m, t o t}}{\rho_{G}^{0} S_{t o t}}
\end{aligned}
$$

donde $\gamma$ representa la relación entre la capacidad calorífica del gas en las condiciones de alimentación (por unidad de volumen de gas), $\left(\rho c_{p}\right)_{G}^{0}$, y la capacidad calorífica total por unidad de volumen de lecho, $\varepsilon_{L}\left(\rho c_{p}\right)_{G}^{0}+\left(1-\varepsilon_{L}\right)\left(\rho c_{p}\right)_{S}$. Adicionalmente, $\Delta T_{R}$ es el máximo salto térmico en el equipo, dado por $T_{S, \max }-T_{G}^{0}$ y $S_{\text {tot }}$ la sección total del equipo. Cabe aclarar que las aproximaciones realizadas en las Ecs. (7.5) y (7.6) llevarán a predecir valores ligeramente mayores que la expresión original, por lo cual resultan más conservativas y, por lo tanto, más adecuadas para el objetivo propuesto.

A partir de las Ecs. (7.5) y (7.6), el volumen necesario para que se desplace el frente de onda, $V_{F}=v_{F} S_{\text {tot }} t_{\text {ciclo }} / 2$, se puede estimar como:

$$
V_{F}=\frac{c_{p G}^{0} G_{m, t o t}}{\left(1-\varepsilon_{L}\right)\left(\rho c_{p}\right)_{S}} \frac{t_{c i c l o}}{2}
$$


Con ello, la cota superior para el volumen del equipo se calcula directamente como $V_{e q, \max }=V_{e q, \min }+V_{F}$.

La Ec. (7.7) sugiere que el volumen necesario del equipo aumentará linealmente con el tiempo de ciclo. Por otro lado, se observa que la velocidad del frente de onda crece al aumentar la porosidad del lecho (Ec. 7.6). Por esta razón, se incluyeron las configuraciones 4 y 5 en el análisis, dado que las mismas mantienen el número de canales por metro cuadrado que poseen las configuraciones 2 y 3 , respectivamente, pero con un mayor espesor de tabique de forma tal que la porosidad sea igual a la de la configuración 1 (67.8\%). De la Tabla 7.2, se puede corroborar que al pasar de la configuración 2 a la 4 (o de la 3 a la 5), se mantiene el factor $K_{V}$, mientras que se aumenta $R_{f}$ (no deseable) y se disminuye $H_{C}$ (deseable). En adición, debido a que para mantener la porosidad en el valor correspondiente a la configuración 1 se debe mantener la relación $\delta_{T} / L_{C}$, cualquiera de las configuraciones de celda modificada presenta también el mismo valor del parámetro $M I F$ que la primera, lo cual resulta beneficioso desde el punto de vista de la resistencia estructural del monolito. De lo anterior, se puede anticipar que las configuraciones modificadas permitirian obtener equipos más compactos que las originales, aun cuando pudieran contener mayor masa total.

Finalmente, teniendo la cota superior de volumen, se puede calcular la cota superior de diámetro de una forma muy sencilla. A partir de la Ec. (3.19), si las propiedades se consideran constantes, se puede verificar que la pérdida de carga resulta proporcional a $V_{e q} / D_{e q}^{4}$. Luego, con los valores de $V_{e q, \min }, D_{e q, \min } \mathrm{y}$ $V_{\text {eq, } \max }$, se puede calcular fácilmente $D_{\text {eq, } \max }$. No obstante, se debe tener presente que en la estimación del diámetro mínimo no se tuvo en cuenta que la pérdida de carga cuando se trabaja con la carga nominal será mayor (a raíz del mayor nivel térmico del lecho). Más aún, recordando que para la condición nominal se propone ajustar el tiempo de ciclo de manera tal que la temperatura máxima no supere los $400^{\circ} \mathrm{C}$, se puede sortear este inconveniente introduciendo la relación $\left(\mu_{G} / \rho_{G}\right)_{T_{i g n}+\Delta T_{a d, \text {,min }}} /\left(\mu_{G} / \rho_{G}\right)_{T_{S, \text { max }}}$, para la estimación de la cota máxima del diámetro. Es decir: 


$$
D_{e q, \max }=D_{e q, \min }\left(\frac{V_{e q, \max }}{V_{e q, \min }}\left(\frac{\mu_{G}}{\rho_{G}}\right)_{T_{S, \max }} /\left(\frac{\mu_{G}}{\rho_{G}}\right)_{T_{i g n}+\Delta T_{a d, \min }}\right)^{1 / 4}
$$

En la Tabla 7.3 se presentaron los valores de $V_{\text {eq, max }}$ y $D_{e q, \max }$, en forma conjunta con los valores de $V_{e q, \min }$ y $D_{e q, \min }$, para una fracción de inerte de $40 \%$ y un tiempo de ciclo de $240 \mathrm{~s}$.

Se puede observar que al aumentar el número de canales por metro cuadrado disminuye el volumen mínimo. Por otro lado, para un mismo número de canales por metro cuadrado (configuraciones 2 y 4 o 3 y 5 ), el volumen mínimo es relativamente insensible a un aumento en el espesor de tabique (presumiblemente debido a que se mantiene el factor $K_{V}$ ) pero, como consecuencia, se requiere de una mayor masa total. En cuanto a las cotas superiores, se observa una disminución en los valores predichos de volumen máximo entre las configuraciones 2 y 4 o entre las 3 y 5 , tal como fuera adelantado.

\subsubsection{Diseños resultantes para cada configuración}

A partir de los criterios de diseño establecidos en la Sección 7.1 y teniendo en cuenta los rangos volcados en la Tabla 7.3, se buscaron las dimensiones del equipo y el tiempo de ciclo nominal para las 5 configuraciones mencionadas anteriormente. Los resultados se vuelcan en la Tabla 7.4, junto con los parámetros considerados como más relevantes, donde $t_{\text {ciclo }}^{*}$ representa el tiempo de ciclo para el cual la emisión global de VOCs es exactamente 9 ppm, correspondiente al valor límite establecido previamente.

A fin de establecer la comparación entre los diseños alcanzados, se debe remarcar una diferencia entre la condición de operación con el mínimo contenido de VOCs y con el nominal, correspondiendo esta última a la situación de trabajo típica del sistema. Considerando que existe siempre un grado de incertidumbre en los resultados (por ejemplo, asociado a la determinación de los parámetros y expresiones cinéticas), el diseño alcanzado deberá poseer el suficiente margen de seguridad para que la operación en las condiciones 
Tabla 7.4: resumen de resultados obtenidos en el dimensionamiento del esquema de inversión de flujo convencional para diferentes configuraciones de celda, según la propuesta de diseño dada en la Sección 7.1. Tiempo de ciclo mínimo: 240 s.

\begin{tabular}{|c|c|c|c|c|c|c|}
\hline \multicolumn{2}{|c|}{$\mathrm{n}^{\circ}$ de configuración } & \multirow{2}{*}{$\begin{array}{c}\mathbf{1} \\
\mathbf{( 2 0 0 / 1 2 . 5 )} \\
5.120\end{array}$} & \multirow{2}{*}{$\begin{array}{c}\begin{array}{c}2 \\
(400 / 7.5)\end{array} \\
3.660\end{array}$} & \multirow{2}{*}{$\begin{array}{c}\begin{array}{c}3 \\
(600 / 4)\end{array} \\
4.340\end{array}$} & \multirow{2}{*}{$\begin{array}{c}\begin{array}{c}4 \\
(400 / 8.8)\end{array} \\
3.385\end{array}$} & \multirow{2}{*}{$\begin{array}{c}5 \\
\mathbf{( 6 0 0 / 7 . 2 )} \\
2.857\end{array}$} \\
\hline & $V_{e q}\left[\mathrm{~m}^{3}\right]$ & & & & & \\
\hline & $L_{e q}[\mathrm{~m}]$ & 1.614 & 1.142 & 1.207 & 0.996 & 0.802 \\
\hline & $D_{e q}[\mathrm{~m}]$ & 2.010 & 2.020 & 2.140 & 2.080 & 2.130 \\
\hline & $W_{I}[\mathrm{~kg}]$ & 4001 & 2401 & 1801 & 2606 & 2174 \\
\hline & $w_{c}[\mathrm{~kg}]$ & 156 & 163 & 251 & 146 & 151 \\
\hline \multirow{3}{*}{ 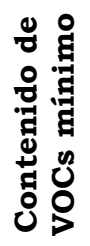 } & $\boldsymbol{T}_{S, \max }\left[{ }^{\circ} \mathbf{C}\right]$ & 273 & 289 & 321 & 285 & 302 \\
\hline & $\left\langle y_{\text {vocs }}\right\rangle[\mathrm{ppm}]$ & 0.992 & 0.999 & 1.005 & 0.998 & 1.003 \\
\hline & $|\Delta \boldsymbol{P}|$ [mbar] & 37.1 & 42.1 & 44.6 & 40.0 & 44.4 \\
\hline \multirow{7}{*}{ 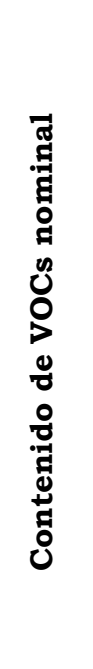 } & $t_{\text {ciclo }}[\mathbf{s}]$ & 240 & 276 & 272 & 270 & 284 \\
\hline & $t_{\text {ciclo, crit }}[\mathbf{s}]$ & 581 & 378 & 323 & 400 & 348 \\
\hline & $\frac{t_{\text {ciclo,crit }}-t_{\text {ciclo }}}{t_{\text {ciclo }}}$ & 1.42 & 0.37 & 0.19 & 0.48 & 0.23 \\
\hline & $t_{c i c l o}^{*}[\mathrm{~s}]$ & 528 & 346 & 290 & 367 & 319 \\
\hline & $\boldsymbol{T}_{S, \max }\left[{ }^{\circ} \mathbf{C}\right]$ & 371 & 398 & 400 & 399 & 396 \\
\hline & $\left\langle y_{\text {vocs }}\right\rangle[\mathrm{ppm}]$ & 0.777 & 0.875 & 1.504 & 0.708 & 0.848 \\
\hline & $|\Delta \boldsymbol{P}|$ [mbar] & 49.4 & 49.8 & 48.9 & 48.9 & 49.8 \\
\hline
\end{tabular}

nominales siempre sea factible. Este margen se puede traducir en la diferencia entre los valores de tiempo de ciclo asociados a tal condición y el valor crítico, relativa al tiempo de ciclo en la condición nominal, informada también en la Tabla 7.4. Por otro lado, se debe tener en cuenta que el mínimo contenido de VOCs fue considerado a los fines de cubrir posibles variaciones en la concentración de VOCs de la corriente a tratar, situación que resulta muy común en procesos de eliminación de contaminantes. Al representar esto un caso límite en sí mismo, no se estudió el rango de tiempos de ciclo posibles para tal con- 
dición. En adición al volumen total del equipo, resulta útil disponer de los valores de masa total de material inerte y catalitico, $W_{I}$ y $W_{C}$, respectivamente, debido a la variación de porosidad entre las distintas configuraciones. Dichos parámetros, que se informan también en la Tabla 7.4, se calculan a partir de:

$$
\begin{aligned}
& W_{I}=\rho_{I} V_{e q}\left[f_{I}\left(1-\varepsilon_{L, I}\right)+\left(1-f_{I}\right)\left(1-\varepsilon_{L, C}^{0}\right)\right] \\
& W_{C}=\rho_{C} V_{e q}\left(1-f_{I}\right)\left(\varepsilon_{L, C}^{0}-\varepsilon_{L, C}\right)
\end{aligned}
$$

donde $\varepsilon_{L, C}^{0}$ representa la porosidad que tendría el lecho sin el depósito catalítico.

En primer lugar, se puede observar que al aumentar el número de celdas, con las configuraciones tomadas de Boger y col. (2004), se logra reducir significativamente la masa total de material a utilizar, principalmente debido a la fuerte disminución de la cantidad de material inerte. No obstante, esto no tiene un correlato directo ni con las dimensiones del equipo (notar que disminuye el volumen total al pasar de la configuración 1 a la 2 , pero vuelve a aumentar de la 2 a la 3), ni con la masa de catalizador (la cual aumenta en todos los casos). Por otro lado, cuando se toman las configuraciones modificadas (4 y 5), se puede observar que la masa total también disminuye respecto de la configuración 1 (aunque la masa de inerte resulta mayor que en las respectivas 2 y 3 , mientras que la de catalizador resulta menor). Sin embargo, se logran obtener equipos sustancialmente más compactos. Por otra parte, en lo que respecta al diámetro del equipo, no se observan cambios significativos para las configuraciones 1,2 y 4, mientras que aumenta para las 3 y 5 , lo cual podría dificultar la correcta distribución de flujo.

En segundo lugar, se debe evaluar qué impacto posee el cambio de configuración sobre la estabilidad del sistema. Al respecto, si se observan los resultados para el contenido de VOCs nominal, puede verse que el aumento del número de celdas genera una disminución del tiempo de inversión crítico en todos los casos (ver valores para las configuraciones 1, 2 y 3, o 1, 4 y 5). Esto es una consecuencia directa de la disminución de la masa total en el equipo. En adición, la diferencia entre el tiempo de ciclo nominal y el crítico 
también disminuye, indicando que la operación del sistema se torna más sensible (y por lo tanto, con mayor tendencia a la inestabilidad). El uso de las configuraciones 4 o 5 en lugar de las 2 y 3 permite mejorar muy levemente esta situación, lo cual es consistente con la disminución del parámetro $H_{C}$ (Tabla 7.2).

Finalmente, debe tenerse presente que el tiempo de ciclo es la principal variable de control que poseerá el sistema una vez construido (e.g. Marín y col., 2010; Cittadini y col., 2001). Al respecto, es importante notar que la disminución de la diferencia entre el tiempo de ciclo resultante para la condición nominal y el crítico (tanto absoluta como relativa ${ }^{15}$ ) se podría relacionar con una mayor dificultad en el control del proceso. Por ello, será deseable que ambos parámetros no sean en extremo pequeños.

\subsubsection{Análisis complementario de los diseños obtenidos}

A fin de complementar los resultados presentados en la Tabla 7.4, se grafica la respuesta de cada uno de los diseños establecidos frente a la variación del tiempo de ciclo para la condición nominal (registrando $T_{S, \max },\left\langle y_{\text {VOCs }}\right\rangle$ y $|\Delta P|$ ), la cual se muestra en la Figura 7.1. En la misma se observa claramente la reducción del rango de tiempos de ciclo que podrían emplearse al aumentar el número de celdas y/o aumentar la porosidad (comparar, por ejemplo, cualquiera de las curvas para la configuración 5 frente a la 3). En consecuencia, la respuesta del sistema se vuelve más abrupta, en especial para la configuración 3, lo que se evidencia en la variación de la emisión de VOCs. Esto último también puede deducirse a partir de la Tabla 7.4, en donde se observa una fuerte disminución de la diferencia entre el tiempo de ciclo seleccionado y el tiempo de ciclo para el cual la emisión de VOCs alcanza el valor de 9 ppm $\left(t_{\text {ciclo }}^{*}\right)$, respecto de la distancia al tiempo de ciclo crítico.

\footnotetext{
15 La diferencia absoluta está íntimamente relacionada con la sensibilidad del sistema (y consecuentemente con la tendencia a la inestabilidad). Por lo tanto, si tal diferencia fuera muy pequeña la respuesta del sistema frente a alguna variación sería presumiblemente abrupta y dificultaría el control del proceso.

Por otro lado, considerando que cualquier estrategia de control se situaría en la condición de trabajo nominal como base, la distancia relativa al tiempo de ciclo en tal situación podría estar relacionada a la capacidad de ajustar con precisión el tiempo de ciclo a una nueva condición.
} 


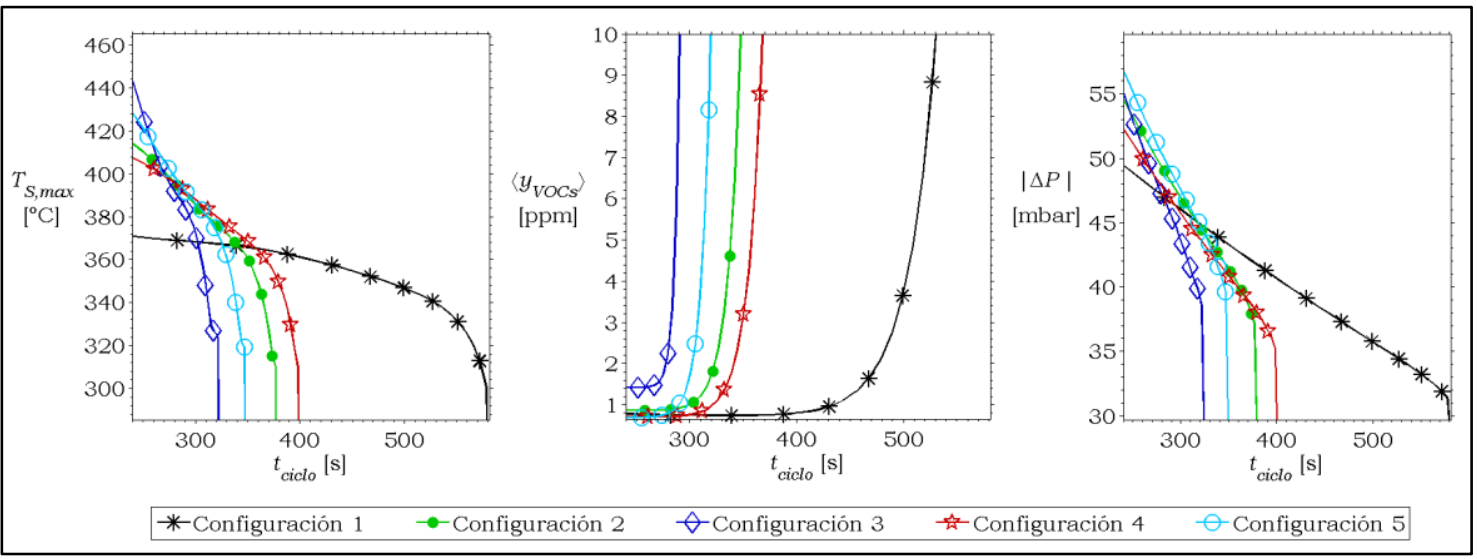

Figura 7.1: respuesta del sistema de inversión de flujo convencional frente a la variación de $t_{\text {ciclo }}$ para el contenido de VOCs nominal y para cada uno de los diseños alcanzados.

En base a la discusión previa, las configuraciones 3 y 5 parecieran no ser recomendables, debido principalmente a que la diferencia relativa entre el tiempo de ciclo crítico y el correspondiente a la condición nominal se vuelve muy desfavorable (ver Tabla 7.4). De las restantes, la primera es la que presenta una respuesta más suave (ver Figura 7.1) y posee un rango de tiempos de ciclo más amplio (ver Figura 7.1 y Tabla 7.4 ). Sin embargo, esto se logra a expensas de una mayor cantidad de material (del orden de 1.5 veces la cantidad utilizada para las configuraciones 2 y 4) y también de dimensiones más grandes. Por otro lado, el equipo con la configuración 4 presenta una respuesta levemente menos pronunciada y permite aumentar la distancia entre el tiempo de ciclo nominal y el tiempo crítico (y lógicamente con el valor correspondiente a una emisión de $9 \mathrm{ppm}$ ), en comparación con los valores obtenidos para la configuración 2. En adición, dicha configuración es la que menos material catalítico requiere. Por lo tanto, se sugiere el uso de la configuración 4 (400/8.8) para el esquema de inversión de flujo convencional.

\subsection{Diseño del esquema rotativo}

Previo a la descripción del diseño para este esquema, es conveniente analizar brevemente cómo influye la fracción de la sección total de flujo destinada a la limpieza de los canales (abreviadamente, fracción de limpieza $-f_{L}-$ ), ya que la misma representa un grado de libertad adicional. Al respecto, cabe recordar que a diferencia de los análisis realizados para el esquema regenera- 
dor-reactor, la fracción de limpieza no fue tenida en cuenta en el Capítulo 5, por considerarse más específica de la etapa de diseño. Esto se debe a que posee un mayor impacto sobre la pérdida de carga para este esquema, debido a que la alimentación a la sección de limpieza corresponde a la salida de la etapa de reacción (ver representación esquemática en la Figura 3.1), mientras que en el regenerador es independiente.

Para realizar el estudio del impacto de la fracción de limpieza, se consideró un intervalo de $f_{L}$ comprendido entre $0 \%$ (sin fracción de limpieza) y $5 \%$. El resto de los parámetros fueron fijados de acuerdo con lo establecido en el Capítulo 5, con excepción del tiempo de ciclo que se fijó en 30 segundos, valor más representativo para este caso. Para mayor facilidad, se reitera en la Tabla 7.5 tal conjunto de parámetros. Los resultados se vuelcan en las Figuras 7.2 y 7.3 .

Tabla 7.5: parámetros utilizados para el estudio del efecto de la fracción de limpieza sobre el esquema de inversión de flujo rotativo.

\begin{tabular}{ccccccc}
\hline $\begin{array}{c}\boldsymbol{L}_{\text {eq }} \\
{[\mathrm{m}]}\end{array}$ & $\begin{array}{c}\boldsymbol{D}_{\text {eq }} \\
{[\mathrm{m}]}\end{array}$ & $\begin{array}{c}\boldsymbol{f}_{\boldsymbol{I}} \\
{[\%]}\end{array}$ & $\begin{array}{c}\boldsymbol{t}_{\text {ciclo }} \\
{[\mathbf{s}]}\end{array}$ & $\begin{array}{c}\Delta \boldsymbol{T}_{\boldsymbol{a d}} \\
{\left[{ }^{\circ} \mathbf{C}\right]}\end{array}$ & $\begin{array}{c}\boldsymbol{N}_{\mathbf{c}} \\
{\left[\mathbf{m}^{-2}\right]}\end{array}$ & $\begin{array}{c}\boldsymbol{\delta}_{\boldsymbol{T}} \\
{[\mathbf{m}]}\end{array}$ \\
\hline 0.815 & 2.5 & {$[25 ; 50 ; 75]$} & 30 & 40 & $3.110^{5}$ & $3.17510^{-4}$ \\
\hline
\end{tabular}

En forma similar a lo ocurrido en el esquema regenerador-reactor, mientras la fracción de limpieza sea baja, la variación del caudal de limpieza para un dado tiempo de ciclo (ver Ec. 4.12) es baja ${ }^{16}$. Como consecuencia, si el valor $f_{L}$ es muy pequeño $(<0.5 \%)$, la velocidad másica será muy elevada y también lo será el impacto de la fracción de limpieza sobre la pérdida de carga total, aspecto que se evidencia en la Figura 7.2 (izquierda). De hecho, para garantizar la obtención de una solución para todos los casos (es decir, poder encontrar el css), se limitó en tales casos el caudal de limpieza para que la pérdida de carga asociada al mismo no supere el $75 \%$ del total (Figura 7.2, derecha). Esto se evidencia para valores de $f_{L}$ menores a $0.2 \%$ únicamente. Para valores mayo-

\footnotetext{
16 Por ejemplo, para una fracción de inerte del 50\%, y considerando el rango de $f_{L}$ comprendido entre $0.2 \%$ y $5 \%$, el caudal de limpieza promedio fue $7.0910^{-2} \mathrm{~kg} / \mathrm{s}$, con una variación del $\pm 5 \%$.
} 
res, el aporte de esta región a la pérdida de carga total decrece rápidamente, alcanzando niveles menores al $30 \%$ a partir de $f_{L} \approx 1.4 \%$.

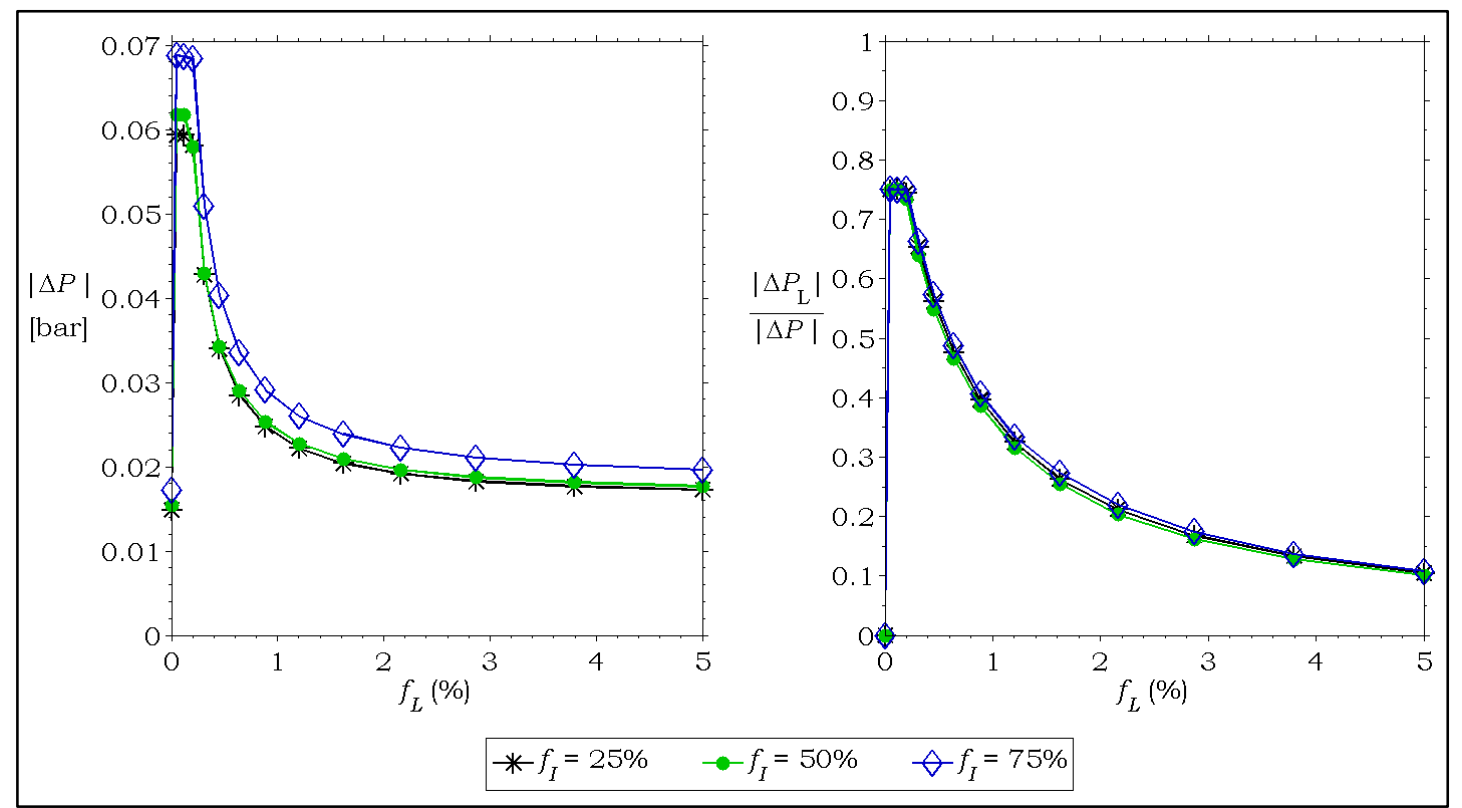

Figura 7.2: efecto de la fracción de limpieza sobre la pérdida de carga en el sistema de inversión de flujo rotativo (configuración 1). $\left|\Delta P_{L}\right|$ representa la pérdida de carga en la región de limpieza.

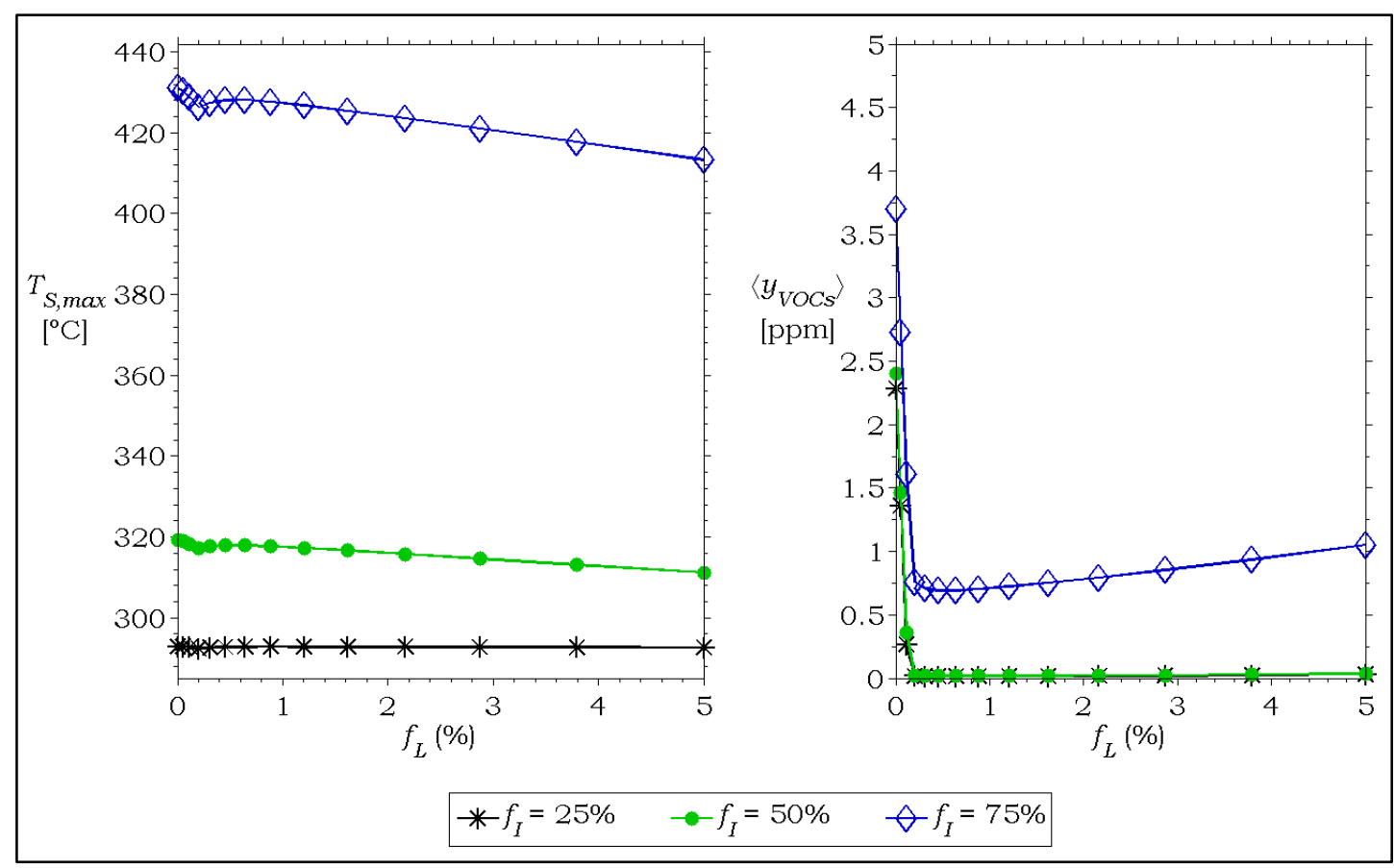

Figura 7.3: efecto de la fracción de limpieza sobre la temperatura máxima y la emisión de VOCs en el sistema de inversión de flujo rotativo (configuración 1). 
Por otro lado, el comportamiento de la temperatura máxima y la emisión de VOCs (Figura 7.3), sigue un patrón completamente análogo al observado y explicado para el sistema regenerador-reactor en el Capítulo 6.

En base a los resultados sobre el efecto de la fracción de limpieza se consideró un valor para la misma de $\mathbf{2} \%$ para todos los casos. Con dicho valor, se garantiza el barrido de los VOCs residuales en los canales, mientras que la pérdida de carga adicional, generada por la inclusión de dicha región, rondará el $25 \%$ del valor total.

Respecto al diseño de este esquema, se propone también el estudio de las 5 configuraciones de celda listadas en la Tabla 7.2. Por otro lado, habiendo fijado el valor de la fracción de limpieza, los grados de libertad disponibles son los mismos que los enunciados en la Sección 7.1. Luego, la propuesta básica de diseño formulada en la Sección 7.1, no se verá afectada y, por lo tanto, solo se enumerarán a continuación los criterios que han de modificarse.

En primer lugar, el tiempo de ciclo mínimo para este esquema queda determinado por la máxima velocidad de rotación admisible. Como valor de referencia, el intervalo de trabajo típico de los intercambiadores rotativos comerciales está comprendido entre 2 a $10 \mathrm{rpm}$, llegando inclusive a los $13 \mathrm{rpm}$ (Regenerative heat recovery with rotary heat exchangers, 2008; Technical conditions for rotary exchangers for heat recovery, s.f.). No obstante, considerando que los procesos y materiales son diferentes, se adoptó como velocidad de rotación máxima de diseño el límite inferior del intervalo mencionado. Esto define el tiempo de ciclo mínimo como $30 \mathbf{s}$.

Por otro lado, el uso de tiempos de ciclos significativamente menores que en el esquema convencional, conduciría a equipos más compactos (ver estimaciones de mínimo y máximo volumen requerido en las Secciones 7.1.1.1 y 7.1.1.2). Teniendo esto presente, para este esquema se fijó la fracción de inerte en un $\mathbf{5 0 \%}$.

Nuevamente quedan saturados todos los grados de libertad enumerados y, sin explicitar la secuencia iterativa, los criterios enunciados se pueden resumir según: 
* Fijar la fracción de limpieza en $2 \%$.

* Fijar la fracción de inerte en 50\%.

* Para el contenido de VOCs mínimo:

- fijar la emisión global en 1 ppm y el tiempo de ciclo en su menor valor (30 s). Determinar el volumen del equipo.

* Para el contenido de VOCs nominal:

^ si para el menor tiempo de ciclo, la temperatura máxima en el lecho resultase menor a $400{ }^{\circ} \mathrm{C}$, fijar el tiempo de ciclo en su valor mínimo (30 s) y determinar el diámetro que permita satisfacer $|\Delta P|=50$ mbar;

$\downarrow$ caso contrario, determinar el diámetro y el tiempo de ciclo que permitan satisfacer en simultáneo $|\Delta P|=50$ mbar y $T_{S, \max }=400{ }^{\circ} \mathrm{C}$.

\subsubsection{Cotas inferior y superior para el volumen y el diáme- tro del equipo}

Si se desprecia el efecto de la fracción de limpieza en el comportamiento térmico del sistema para los casos limites expuestos anteriormente (Sección 7.1.1), la estimación de las cotas inferiores y superiores podrá realizarse directamente en base a lo discutido en las Secciones 7.1.1.1 y 7.1.1.2. No obstante, según lo que se discutió al comienzo de la Sección 7.2, para un mismo volumen y diámetro, la presencia de una fracción de limpieza del 2\% aumentará la pérdida de carga en $\approx 25 \%$ (ver Figura 7.3 , derecha). Considerando esto, se sugiere disminuir el valor de $|\Delta P|$ utilizado para la estimación del mínimo diámetro al $75 \%$ del valor original (es decir, adoptar el valor de 37.5 mbar).

Finalmente, recordando que la fracción de inerte adoptada para este sistema es de $50 \%$ y que el tiempo de ciclo mínimo es de $30 \mathrm{~s}$, se obtienen los rangos volcados en la Tabla 7.6. 
Tabla 7.6: cotas inferiores y superiores para el volumen y diámetro del equipo en el sistema de inversión de flujo rotativo.

\begin{tabular}{ccc}
\hline $\mathbf{n}^{\mathbf{0}}$ de configuración & $\begin{array}{c}\left(\boldsymbol{V}_{T, \min }-\mathbf{V}_{T, \max }\right) \\
{\left[\mathbf{m}^{\mathbf{3}}\right]}\end{array}$ & $\begin{array}{c}\left(\boldsymbol{D}_{\boldsymbol{T}, \min }-\boldsymbol{D}_{T, \max }\right) \\
{[\mathbf{m}]}\end{array}$ \\
\hline $\mathbf{1}$ & $3.98-4.22$ & $1.92-2.13$ \\
\hline $\mathbf{2}$ & $2.10-2.37$ & $1.89-2.13$ \\
\hline $\mathbf{3}$ & $1.43-1.83$ & $1.79-2.08$ \\
\hline $\mathbf{4}$ & $2.11-2.35$ & $1.95-2.19$ \\
\hline $\mathbf{5}$ & $1.44-1.68$ & $1.97-2.23$ \\
\hline
\end{tabular}

\subsubsection{Diseños resultantes para cada configuración}

En la Tabla 7.7 se vuelcan los resultados del dimensionamiento del sistema de inversión de flujo rotativo, de acuerdo a lo descrito en las secciones anteriores.

En primer lugar, se puede observar que efectivamente, cualquiera sea la configuración elegida, se consiguen diseños significativamente más compactos que en el esquema de inversión de flujo convencional. Lógicamente, en consonancia con el menor volumen (y masa) de equipo, los tiempos de ciclo críticos para el contenido nominal se ven severamente disminuidos en comparación con el anterior. No obstante, si se considera la diferencia absoluta entre el tiempo de ciclo crítico (o con $t_{\text {ciclo }}^{*}$ ) y el seleccionado para la operación nominal, se puede corroborar que no hay diferencias apreciables entre este esquema y el otro (excepto para las configuraciones 3 y 5 que resultan significativamente mayores para el esquema rotativo). Por otro lado, dicha diferencia en términos relativos al nominal resulta sustancialmente mayor.

En segundo lugar, se aprecia que en este esquema el aumento del número de celdas permite disminuir significativamente el volumen del equipo en general. Por otro lado, en el caso de las configuraciones 4 y 5 frente a las 2 y 3 , no se observa un cambio importante en el volumen, como sí sucedia con los diseños del esquema de inversión de flujo convencional. Como consecuencia, la 
diferencia entre la masa total entre las configuraciones 4 y 5 frente a las 2 y 3 en el esquema rotativo es ligeramente mayor que en el convencional.

Tabla 7.7: resumen de resultados obtenidos en el dimensionamiento del esquema de inversión de flujo rotativo para diferentes configuraciones de celda, según la propuesta de diseño dada en la Sección 7.2. Tiempo de ciclo mínimo: $30 \mathrm{~s}$.

\begin{tabular}{|c|c|c|c|c|c|c|}
\hline \multicolumn{2}{|c|}{$n^{\circ}$ de configuración } & \multirow{2}{*}{$\frac{\begin{array}{c}1 \\
(200 / 12.5)\end{array}}{4.080}$} & \multirow{2}{*}{$\begin{array}{c}\mathbf{2} \\
\mathbf{( 4 0 0 / 7 . 5 )} \\
2.199\end{array}$} & \multirow{2}{*}{$\frac{\begin{array}{c}3 \\
(600 / 4)\end{array}}{1.621}$} & \multirow{2}{*}{$\begin{array}{c}\frac{4}{(400 / 8.8)} \\
2.192\end{array}$} & \multirow{2}{*}{$\begin{array}{c}5 \\
(600 / 7.2)\end{array}$} \\
\hline & $V_{e q}\left[\mathrm{~m}^{3}\right]$ & & & & & \\
\hline & $L_{e q}[\mathrm{~m}]$ & 1.286 & 0.729 & 0.610 & 0.691 & 0.485 \\
\hline & $D_{e q}[\mathrm{~m}]$ & 2.010 & 1.960 & 1.840 & 2.010 & 2.010 \\
\hline & $W_{I}[\mathbf{k g}]$ & 3207 & 1458 & 687 & 1702 & 1183 \\
\hline & $W_{c}[\mathbf{k g}]$ & 103 & 82 & 78 & 79 & 68 \\
\hline \multirow{3}{*}{ 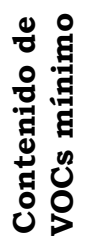 } & $T_{s, \max }\left[{ }^{\circ} \mathbf{C}\right]$ & 270 & 277 & 282 & 278 & 282 \\
\hline & $\left\langle y_{\text {vocs }}\right\rangle[\mathrm{ppm}]$ & 0.998 & 1.003 & 1.005 & 0.996 & 1.003 \\
\hline & $|\Delta \boldsymbol{P}|$ [mbar] & 45.3 & 42.3 & 45.4 & 42.9 & 42.9 \\
\hline \multirow{7}{*}{ 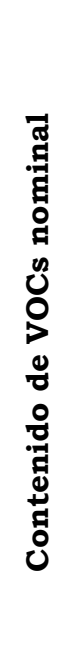 } & $t_{\text {ciclo }}[\mathbf{s}]$ & 80 & 58 & 54 & 70 & 66 \\
\hline & $t_{c i c l o, c r i t}[\mathbf{s}]$ & 401 & 186 & 93 & 214 & 152 \\
\hline & $\frac{t_{\text {ciclo, crit }}-t_{\text {ciclo }}}{t_{\text {ciclo }}}$ & 4.01 & 2.21 & 0.72 & 2.06 & 1.30 \\
\hline & $t_{\text {ciclo }}^{*}[\mathrm{~s}]$ & 346 & 162 & 83 & 187 & 132 \\
\hline & $T_{S, \max }\left[{ }^{\circ} \mathbf{C}\right]$ & 398 & 397 & 398 & 395 & 398 \\
\hline & $\left\langle y_{\text {vocs }}\right\rangle[\mathbf{p p m}]$ & 0.003 & 0.003 & 0.039 & 0.004 & 0.009 \\
\hline & $|\Delta \boldsymbol{P}|$ [mbar] & 50.0 & 49.8 & 49.4 & 49.7 & 49.4 \\
\hline
\end{tabular}

Por último, debe tenerse presente que en este esquema existe un compromiso entre la longitud y el diámetro del equipo. Por un lado, un diámetro pequeño permitirá una mejor distribución de flujo (lo cual favorece a la configuración 3). No obstante, exceptuando a la configuración 3, los demás diseños presentan valores de diámetros muy cercanos entre sí. Por otro lado, recor- 
dando que el equipo debe rotar sobre un eje, será deseable que la longitud del equipo resulte pequeña a fin de disminuir el esfuerzo sobre el eje del rotor y los cojinetes respectivos (lo cual favorece a la configuración 5).

\subsubsection{Análisis complementario de los diseños obtenidos}

Al igual que lo expuesto para el sistema de inversión de flujo convencional, se graficó la respuesta de cada uno de los diseños establecidos frente a la variación del tiempo de ciclo para la condición con el contenido de VOCs nominal (Figura 7.4). Nuevamente, se observa que al aumentar el número de celdas se reduce apreciablemente el tiempo de ciclo crítico así como la diferencia entre el tiempo de ciclo en la condición nominal y el crítico, tanto en términos absolutos como relativos (ver Tabla 7.7). Estos efectos son particularmente marcados para la configuración 3, lo cual es consistente con el elevado valor del parámetro $H_{C}$ (ver Tabla 7.2) y la disminución de la masa total en el equipo (ver Tabla 7.7). Por esta razón, no pareciera ser aconsejable el uso de tal configuración.

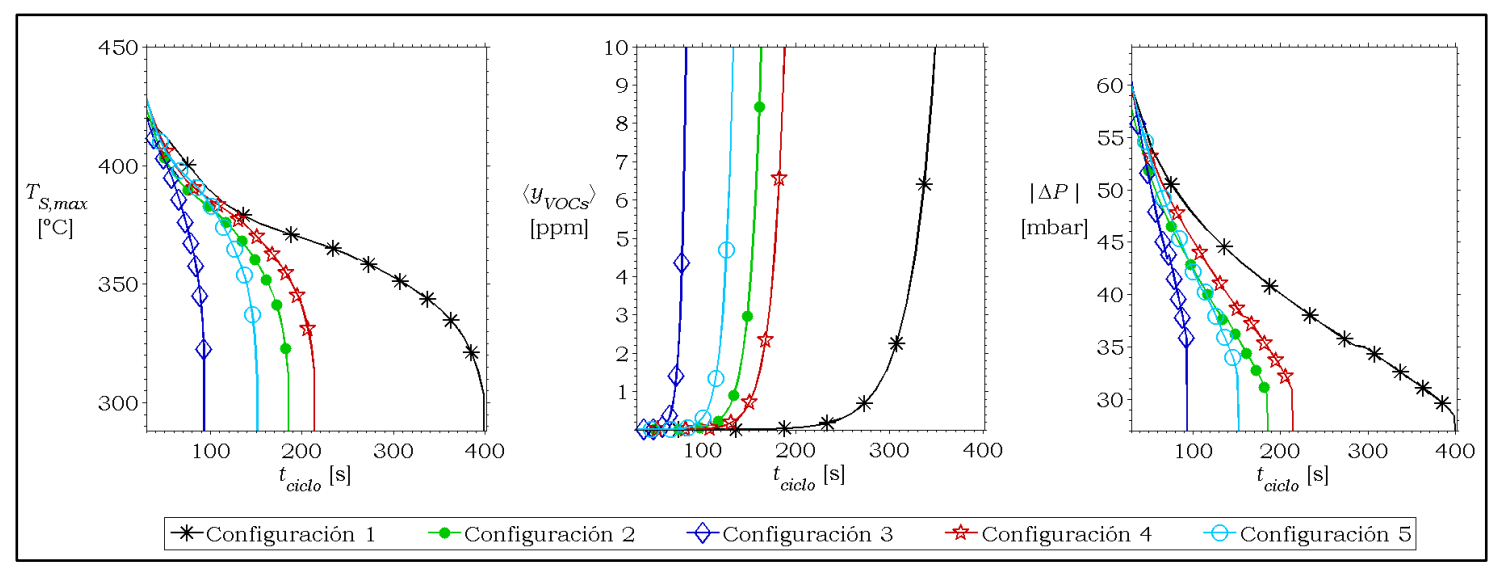

Figura 7.4: respuesta del sistema de inversión de flujo rotativo frente a la variación de $t_{\text {ciclo }}$ para el contenido de VOCs nominal y para cada uno de los diseños alcanzados.

En cuanto a la configuración 1, si bien la misma es la que presenta el rango de tiempos de ciclo más amplio (y la respuesta más "suave"), el diseño alcanzado requiere al menos el doble de masa (y volumen) que los diseños restantes, por lo que nuevamente tampoco parece ser una alternativa adecuada. 
De las alternativas restantes, las correspondientes a las configuraciones $2 \mathrm{y}$ 4 no presentan diferencias significativas en sus dimensiones entre sí, pero se puede notar nuevamente una mejora en el tiempo de ciclo crítico al haber ajustado la porosidad de la configuración 4 a un valor menor. Contrariamente, la diferencia relativa entre el tiempo de ciclo crítico y el correspondiente a la condición nominal se ve ligeramente desfavorecida. No obstante, ambas alternativas presentan resultados similares y parecieran ser adecuadas.

Por otro lado, a diferencia de lo encontrado para el esquema de inversión de flujo convencional, la diferencia relativa entre el tiempo de ciclo crítico y el correspondiente a la condición nominal para la configuración 5 adopta aún valores adecuados (1.30). De esta manera, se obtiene un ahorro significativo de material inerte y catalítico, así como una importante disminución en el volumen del equipo, respecto de las configuraciones 2 y 4 . Más aún, como fuera señalado al comienzo de la Sección 7.2.2, la configuración 5 se ve favorecida por la menor longitud del equipo (ver Tabla 7.7), lo que permite disminuir el esfuerzo sobre el eje del rotor y los cojinetes respectivos.

Por lo expuesto anteriormente, se sugiere el uso de la configuración 5 (600/7.2) para el sistema de inversión de flujo rotativo.

\subsection{Conclusiones y comentarios finales}

Como paso fundamental para establecer una estrategia de diseño, se identificaron los grados de libertad de cada uno de los esquemas de inversión descritos en el Capítulo 3. Adicionalmente, en base al conocimiento del comportamiento de estos esquemas frente a la variación de diferentes parámetros, estudiados en el Capítulo 5, se fijó el valor de la fracción de inerte en un 40\% para el esquema convencional y en un $50 \%$ para el rotativo.

Por otro lado, como fuera mencionado en el Capítulo 2, se pretende tratar una corriente cuyo contenido de VOCs pueda disminuir hasta la mitad de su valor nominal. Considerando que esta situación es la más crítica desde el punto de vista del dimensionamiento de los equipos, se tomó la misma como condición para definir el volumen total en cada esquema, exigiendo una emisión promedio de VOCs de 1 ppm y asumiendo que para ese caso se operaría con el tiempo de ciclo mínimo. Para el esquema convencional, se fijó este últi- 
mo en base a una mínima vida útil esperable de las válvulas ( 2 años, que equivale a un tiempo de ciclo de 240 s). Para el esquema rotativo, se consideró el rango típico de velocidades angulares en los regeneradores actuales, seleccionando el menor valor de dicho rango por ser una elección más conservadora ( $2 \mathrm{rpm}$, que equivale a un tiempo de ciclo de $30 \mathrm{~s}$ ).

En adición, para el esquema rotativo se estudió el efecto de la fracción de limpieza sobre la operación del mismo, especialmente centrada en el efecto sobre la pérdida de carga. A partir de los resultados obtenidos, se fijó dicho parámetro en un $2 \%$ para todos los casos.

Finalmente, la definición de la pérdida de carga admisible y de la temperatura máxima en el lecho permitió especificar el diámetro del equipo y el tiempo de ciclo nominal.

Por otro lado, se limitó el estudio a cinco variantes de configuración de celda, todas en base a monolitos de canales cuadrados, correspondiendo tres de ellas a alternativas comerciales dadas en Boger y col. (2004).

Habiendo definido una estrategia de diseño, se buscaron las dimensiones de ambos esquemas para cada una de las configuraciones. Tanto para el esquema convencional como para el rotativo, la configuración con 600 celdas por pulgada cuadrada y un espesor de tabique de 4 milésimas de pulgada (configuración 3), no resultó adecuada debido a que presenta cambios muy pronunciados frente a la variación del tiempo de ciclo y el rango de operación posible resulta muy estrecho (lo que presumiblemente dificultaría su control una vez instalado el equipo). Por el contrario, la configuración con 200 celdas por pulgada cuadrada (configuración 1) tampoco resultó adecuada, ya que requiere un volumen y masa de material inerte significativamente mayor a las otras opciones (para el esquema convencional, la diferencia con la configuración sugerida ronda el 52\%, mientras que para el esquema rotativo dicha diferencia se eleva al 165\%).

Por otro lado, la masa de catalizador necesaria no varió apreciablemente para las distintas configuraciones en el esquema de inversión de flujo convencional (exceptuando a la configuración 3), mientras que en el esquema rotativo se observó una disminución al aumentar el número de celdas y/o disminuir la porosidad. 
De las restantes opciones, las tres podrian ser aptas, existiendo un evidente compromiso entre la disminución de la masa y volumen requeridos frente a la disminución del rango de tiempos de ciclo observada (tanto en términos absolutos como relativos). En base a los resultados obtenidos, se sugirió el diseño con la configuración 4 (400/8.8) para el esquema de inversión de flujo convencional y la configuración $5(600 / 7.2)$ para el rotativo. 


\section{Capítulo 08}

\section{- Diseño del sistema regenerador-reactor}

- Análisis de grados de libertad y estrategia de diseño

- Justificación de la elección de la temperatura de alimentación al reactor

- Diseños resultantes para cada configuración 

Al igual que lo realizado en el Capítulo 7, se pretende alcanzar un diseño para el esquema regenerador-reactor que permita tratar la corriente de aire contaminada con VOCs detallada en el Capítulo 2, cuyas características se repiten nuevamente en la Tabla 8.1.

Se debe tener presente que este esquema presenta algunas similitudes con los sistemas de inversión de flujo, principalmente en lo que respecta a la emisión residual de VOCs en condiciones normales de operación. Por esta razón, es esperable que se mantengan algunos de los criterios de diseño establecidos en el capítulo anterior. No obstante, también se deben contemplar ciertas diferencias, siendo quizás la más evidente la posibilidad de ajustar las dimensiones de cada equipo (regenerador y reactor) en forma independiente. Como se verá, esto se traduce en más grados de libertar para este esquema.

Otro aspecto que se discute en el presente capitulo es la influencia de la temperatura de alimentación al reactor. Si bien esto fue estudiado en el Capítulo 6 (Sección 6.2, donde se discutió el efecto de la caída en la actividad del catalizador), el análisis se llevó a cabo desde el punto de vista de la operación del sistema. Considerando que la temperatura de alimentación al reactor es clave para el diseño del mismo, se estudia el efecto de tal parámetro, pero desde una perspectiva orientada al diseño.

Tabla 8.1: características de la corriente a tratar.

\begin{tabular}{|c|c|c|c|c|c|c|}
\hline \multirow[t]{2}{*}{$\begin{array}{c}\boldsymbol{G}_{m, t o t} \\
{[\mathbf{k g} / \mathbf{s}]}\end{array}$} & $\begin{array}{c}\boldsymbol{P}^{0} \\
\text { [bar] }\end{array}$ & $\begin{array}{c}T_{G}^{0} \\
{\left[{ }^{\circ} \mathbf{C}\right]}\end{array}$ & \multicolumn{2}{|c|}{$\begin{array}{c}y_{j, G}^{0} \\
\text { (minima) } \\
\text { [ppm] }\end{array}$} & \multicolumn{2}{|c|}{$\begin{array}{c}y_{j, G}^{0} \\
\text { (nominal) } \\
\text { [ppm] }\end{array}$} \\
\hline & & & $\mathrm{AE}$ & Et & $\mathrm{AE}$ & $\mathrm{Et}$ \\
\hline 12.7 & & & 307 & 125 & 615 & 250 \\
\hline
\end{tabular}

\subsection{Análisis de grados de libertad y estrategia de diseño}

Para este esquema se propone también el estudio de las cinco configuraciones de celda presentadas en el Capítulo 7 (Tabla 7.2) y repetidas en la Tabla 8.2 (el significado de los parámetros indicados en la misma es el ya definido en el Capítulo 7). 
Tabla 8.2: configuraciones de celda y propiedades de las mismas.

\begin{tabular}{|c|c|c|c|c|c|c|c|c|c|}
\hline $\mathbf{N}^{\circ}$ & $\begin{array}{r}10^{-5} N_{C} \\
{\left[\mathrm{~m}^{-2}\right]}\end{array}$ & $\begin{array}{c}10^{4} \delta_{T} \\
{[\mathrm{~m}]}\end{array}$ & $\varepsilon_{L}$ & $\begin{array}{c}a_{v} \\
{\left[\mathbf{m}^{-1}\right]}\end{array}$ & $\begin{array}{c}10^{3} d_{h} \\
{[\mathrm{~m}]}\end{array}$ & $\begin{array}{r}10^{-7} R_{f} \\
{\left[\mathrm{~m}^{-2}\right]}\end{array}$ & $\begin{array}{c}10^{-6} K_{V} \\
{\left[\mathrm{~m}^{-2}\right]}\end{array}$ & $\begin{array}{c}10^{-7} H_{C} \\
{\left[\mathrm{~m}^{-2}\right]}\end{array}$ & $10^{2} M I F$ \\
\hline 1 & $\begin{array}{c}3.1 \\
(200)\end{array}$ & $\begin{array}{c}3.18 \\
(12.5)\end{array}$ & 0.678 & 1833 & 1.48 & 1.08 & 4.96 & 1.54 & 3.80 \\
\hline 2 & $\begin{array}{c}6.2 \\
(400)\end{array}$ & $\begin{array}{l}1.91 \\
(7.5)\end{array}$ & 0.722 & 2677 & 1.08 & 1.90 & 9.92 & 3.57 & 2.65 \\
\hline 3 & $\begin{array}{c}9.3 \\
(600)\end{array}$ & $\begin{array}{c}1.02 \\
(4)\end{array}$ & 0.814 & 3480 & 0.94 & 2.25 & 14.9 & 7.98 & 1.06 \\
\hline 4 & $\begin{array}{c}6.2 \\
(400)\end{array}$ & $\begin{array}{l}2.25 \\
(8.8)\end{array}$ & 0.678 & 2593 & 1.05 & 2.16 & 9.92 & 3.08 & 3.80 \\
\hline 5 & $\begin{array}{c}9.3 \\
(600)\end{array}$ & $\begin{array}{l}1.83 \\
(7.2)\end{array}$ & 0.678 & 3176 & 0.85 & 3.24 & 14.9 & 4.62 & 3.80 \\
\hline
\end{tabular}

Asumiendo entonces que se conoce la configuración de celda y teniendo presente la descripción del sistema regenerador-reactor dada en el Capítulo 3, se pueden identificar 7 grados de libertad para el mismo. Estos son:

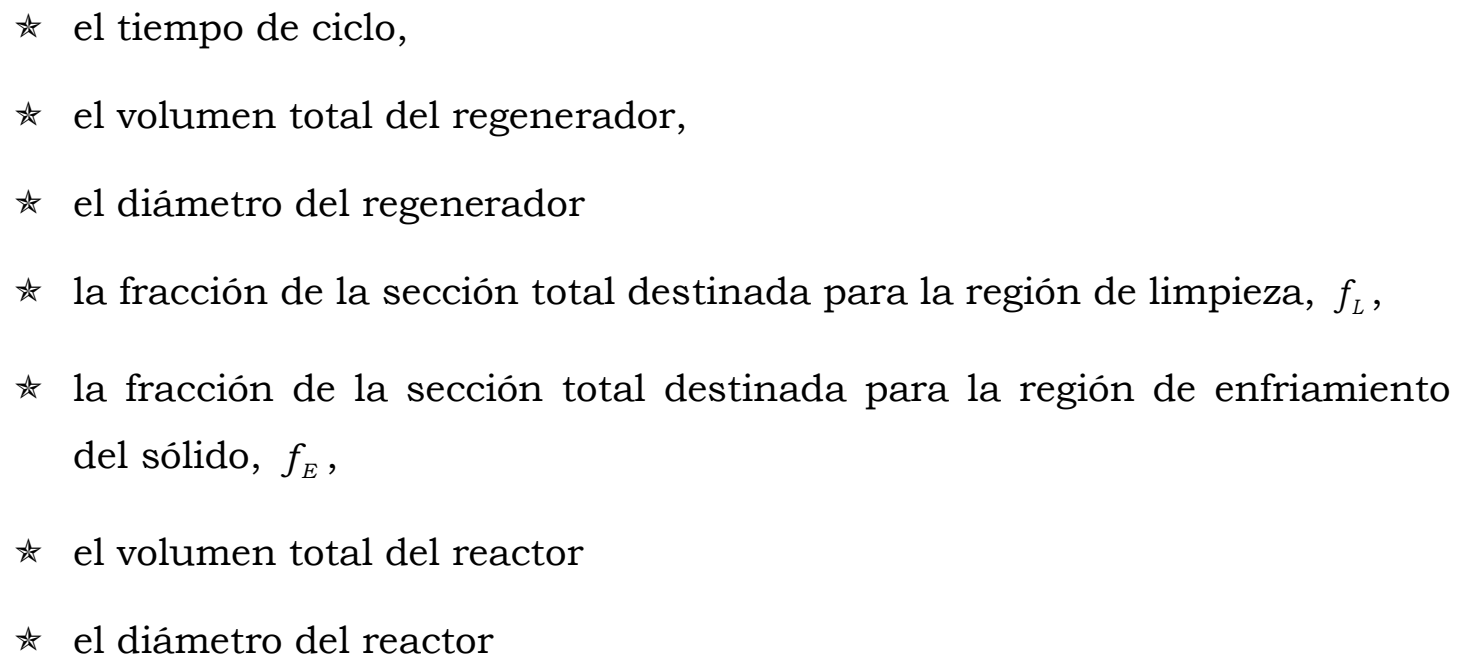

En primer lugar, al igual que en los sistemas de inversión de flujo, cabe aclarar que el tiempo de ciclo posee un rol diferente al resto de las variables. Al ser una variable operativa, el valor de tal parámetro podrá ser diferente para las condiciones nominales o de mínimo contenido de VOCs, lo que permite abarcar el rango propuesto, sujeto a una serie de condiciones determinadas.

En segundo lugar, puede preverse que debido a la mayor cantidad de grados de libertad, la estrategia de diseño para este sistema sea diferente de la 
adoptada para los esquemas de inversión de flujo. Más aún, en los esquemas de inversión de flujo no se puede establecer una diferenciación directa entre las regiones con material catalítico e inerte (recordar que el propio material catalítico participa del intercambio regenerativo), mientras que en este caso ambos equipos (regenerador y reactor) están nítidamente identificados.

Teniendo en cuenta que la búsqueda de un diseño óptimo cae fuera de los alcances propuestos para el presente estudio, la estrategia adoptada consiste nuevamente en saturar (parcial o totalmente) los grados de libertad mencionados, fijando por ejemplo los valores de algunas variables de "salida" (e.g., la pérdida de carga total o la temperatura de alimentación al reactor).

Para tal fin, la cota de emisión global de 9 ppm (Capítulo 2) no será adecuada. Como fuera mencionado en el Capítulo 7 (y estudiado en particular para este esquema en el Apéndice 6.1), esto se debe a que los valores de emisión de VOCs en los esquemas de tipo autotérmico son muy pequeños para condiciones alejadas del límite entre los estados apagados y encendido. Por esta razón, el volumen del reactor, $V_{R}$, se ajustará para la condición de mínimo contenido de VOCs (condición más crítica), exigiendo que la emisión en el css sea de

\section{1 ppm.}

En lo que respecta al volumen del regenerador, $V_{\text {Reg }}$, el mismo se ajustará para obtener una temperatura de entrada al reactor, $T_{G}^{0, R}$, de $280{ }^{\circ} \mathbf{C}$, para la condición de mínimo contenido de VOCs (menor fuerza impulsora) y utilizando el menor tiempo de ciclo posible. La justificación del valor de $T_{G}^{0, R}$ se discute en la Sección 8.2.

Al igual que para el esquema de inversión de flujo rotativo, el tiempo de ciclo mínimo para este esquema queda determinado por la máxima velocidad de rotación admisible. Como valor de referencia, el intervalo de trabajo típico de los intercambiadores rotativos comerciales está comprendido entre 2 a $10 \mathrm{rpm}$ (Regenerative heat recovery with rotary heat exchangers, 2008; Technical conditions for rotary exchangers for heat recovery, s.f.). No obstante, considerando que los materiales son diferentes (los regeneradores ofrecidos por tales empresas utilizan aluminio), se adoptó como velocidad de rotación máxima el límite inferior del intervalo mencionado. Con esto se tendrá en general $t_{\text {ciclo }} \geq 30 \mathrm{~s}$, definiendo el tiempo de ciclo mínimo como 30 s. 
Por otro lado, considerando el análisis realizado en el Capítulo 6 sobre el efecto de la fracción de limpieza, la misma se fijó en un $\mathbf{1 \%}$. Asimismo, la fracción de enfriamiento se fijó en un $\mathbf{4 9 \%}$ (es decir, $f_{L}+f_{E}=1 / 2$ ).

Con la cantidad y tipo de restricciones enunciadas hasta aquí aún no es posible anular completamente los grados de libertad señalados $\mathrm{y}$, consecuentemente, no podrá establecerse un único diseño. Resta por lo tanto definir restricciones adicionales que permitan fijar los diámetros de los equipos y el tiempo de ciclo para la condición nominal (sujeto a $t_{\text {ciclo }} \geq 30 \mathrm{~s}$ ).

Para ajustar el diámetro de ambos equipos, se exige que la pérdida de carga total no supere los $\mathbf{5 0}$ mbar en ningún caso (ni para el mínimo contenido de VOCs, ni para el nominal). Como fuera señalado en el Capítulo 7, en la condición de trabajo nominal es esperable que el nivel térmico sea mayor y consecuentemente también lo sea la pérdida de carga (la relación $\mu_{G} / \rho_{G}$ aumenta con la temperatura). Como consecuencia, los diámetros de los equipos se ajustarán de forma tal que la pérdida de carga, para el contenido de VOCs nominal, sea de 50 mbar $^{17}$. No obstante, esta condición no resulta suficiente, debiendo establecer otra que permita vincular ambos diámetros. Tal condición viene dada por la relación:

$$
D_{R}=D_{\text {Reg }}\left(0.42 \frac{V_{R}}{V_{R e g}}\right)^{1 / 4}
$$

donde $D_{\text {Reg }}$ y $D_{R}$ son los diámetros del regenerador y del reactor, respectivamente.

La relación (8.1) se obtiene igualando la Ec. (3.19) para cada equipo, evaluando la relación $\mu_{G} / \rho_{G}$ a la temperatura promedio en cada uno, esto es:

$$
\frac{1}{4}\left[\mu_{G}\left(T_{m, \max }^{R}\right) / \rho_{G}\left(T_{m, \max }^{R}\right)\right] /\left[\mu_{G}\left(T_{m, \max }^{R e g}\right) / \rho_{G}\left(T_{m, \max }^{R e g}\right)\right] \approx 0.42
$$

17 Nótese que las restricciones que permitirán ajustar el volumen y diámetro de los equipos quedan definidas nuevamente en condiciones diferentes, lo cual conduce a un cálculo de tipo iterativo. 
siendo $\quad T_{m, \max }^{R e g}=\frac{T_{G}^{0}+T_{G, \max }^{0, R}+\Delta T_{a d} / 2}{2}=\frac{\left(T_{G}^{0}+T_{G, \max }^{0, R}\right) / 2+\left(T_{G}^{0}+T_{G, \max }^{0, R}+\Delta T_{a d}\right) / 2}{2}, \quad$ la temperatura media (aritmética) esperable en el regenerador para la condición de operación con el contenido nominal de VOCs (con $T_{G, \max }^{0, R}=T_{S, \max }-\Delta T_{a d}$ ) y $T_{m, \max }^{R}=T_{S, \max }-\Delta T_{a d} / 2$, la temperatura media (aritmética) en el reactor. Adicionalmente, $T_{S, \max }$ corresponde a la máxima temperatura admisible, que se discute en el párrafo siguiente. Si bien tal relación no garantizará la igualdad exacta de $|\Delta P|$ en cada equipo (debido a las simplificaciones sobre los valores promedio del cociente $\mu_{G} / \rho_{G}$ ), permite obtener una pérdida de carga similar en cada uno.

Por último, bajo el mismo concepto expuesto en el Capítulo 7, será deseable limitar la temperatura máxima en el lecho catalítico (en este caso correspondiente a la salida del reactor) a fin de evitar la temprana desactivación del catalizador utilizado. Manteniendo la elección realizada en el capítulo anterior, se adoptó un valor de temperatura máxima admisible en el lecho de $400{ }^{\circ} \mathbf{C}$ (es decir, $T_{S, \max } \leq 400^{\circ} \mathrm{C}$ ). Puesto que la mayor elevación de temperatura se encontrará para la condición nominal, se exige para tal caso $T_{S, \max }=400{ }^{\circ} \mathrm{C}$. Supuesto estén definidas el resto de las variables, se podrá emplear el grado de libertad correspondiente al tiempo de ciclo (sujeto a $t_{\text {ciclo }} \geq 30 \mathrm{~s}$ ) para satisfacer tal condición. Cabe aclarar que, dependiendo de las dimensiones adoptadas (especialmente la del regenerador, fijada a través de la temperatura de alimentación al reactor), podría resultar $T_{S, \max }<400^{\circ} \mathrm{C}$ aún para el mínimo tiempo de ciclo. En tal caso, se adoptará $t_{\text {ciclo }}=30 \mathrm{~s}$ para la condición nominal.

A partir de los criterios expuestos en los párrafos precedentes, quedan saturados todos los grados de libertad enumerados para este sistema. Así, sin explicitar la secuencia iterativa, los criterios enunciados se pueden resumir según:

* Fijar la fracción de limpieza en un 1\%.

* Fijar la fracción de enfriamiento en un 49\%.

* Para el contenido de VOCs mínimo: 
^ fijar la emisión global en 1 ppm, el tiempo de ciclo en su menor valor (30 s) y la temperatura de alimentación al reactor en $280^{\circ} \mathrm{C}$. Determinar el volumen del regenerador y del reactor.

* Para el contenido de VOCs nominal, fijando la relación entre el diámetro del reactor y del regenerador según $D_{R} / D_{\text {Reg }}=\left(0.42 V_{R} / V_{R e g}\right)^{1 / 4}$ :

- si para el menor tiempo de ciclo, la temperatura a la salida del reactor (valor máximo) resultase menor a $400{ }^{\circ} \mathrm{C}$, fijar el tiempo de ciclo en su valor mínimo (30 s) y determinar el diámetro del regenerador que permita satisfacer $|\Delta P|=50$ mbar;

- caso contrario, determinar el diámetro del regenerador y el tiempo de ciclo que permitan satisfacer en simultáneo $|\Delta P|=50$ mbar y $T_{S, \max }=400^{\circ} \mathrm{C}$.

\subsubsection{Determinación de los rangos de búsqueda}

En forma similar a lo expuesto para ambos sistemas de inversión de flujo, se pueden establecer cotas inferiores y superiores para el volumen y diámetro de cada equipo. Sin embargo, el procedimiento difiere respecto al presentado en el Capítulo 7, debiéndose considerar ambos equipos por separado.

\subsubsection{Cotas inferiores y superiores para el regenerador}

En el caso del regenerador, la cota inferior de volumen, $V_{R e g, \min }$, y diámetro, $D_{\text {Reg,min }}$, puede obtenerse considerando el caso con $t_{\text {ciclo }} \rightarrow 0$ (a través de las Ecs. A3.3.1 a A3.3.3, presentadas en el Apéndice 3.3), procedimiento que será iterativo, debido a la dependencia de los coeficientes de transferencia con la velocidad. A partir de la Ec. (3.19), para cada valor de $V_{\text {Reg,min }}$ en dicha iteración, (despreciando la presencia de la fracción de limpieza, considerando que la pérdida de carga en el regenerador corresponde a la mitad de la total y evaluando la relación $\mu_{G} / \rho_{G}$ a la temperatura media en el equipo), el diámetro del regenerador resulta: 


$$
D_{\text {Reg,min }}=\left[\frac{8(f \mathrm{Re}) R_{f} G_{m, t o t} V_{R e g, \min }}{\pi^{2}|\Delta P| / 2}\left(\frac{\mu_{G}}{\rho_{G}}\right)_{T_{m}^{\text {Reg }}}\right]^{1 / 4}
$$

donde $T_{m}^{R e g}=\left[T_{G}^{0}+T_{G}^{0, R}+\Delta T_{a d} / 2\right] / 2$ es la temperatura media (aritmética) correspondiente al caso con menor contenido de VOCs.

Para la cota superior, se debe tener presente que para cualquier tiempo de ciclo no nulo la temperatura del sólido presentará un perfil variable en el tiempo, formándose también un frente de onda (cuyo desplazamiento disminuye la capacidad de intercambio del mismo). Un razonamiento en términos de un lecho de longitud infinita en el cual ingresa una corriente a menor temperatura permite establecer la velocidad a la cual se movería dicho frente, en forma completamente análoga al caso del sistema de inversión de flujo, siendo:

$$
v_{F}=\frac{G_{m, t o t} c_{p G}}{\left(1-\varepsilon_{L}\right)\left(\rho c_{p}\right)_{S}\left(S_{t o t, R e g} / 2\right)}
$$

donde $\left(1-\varepsilon_{L}\right)\left(\rho c_{p}\right)_{S}$ representa la capacidad calorífica del sólido por unidad de volumen de lecho y $S_{\text {tot,Reg }}$ la sección total del regenerador. Notar que esta expresión resulta equivalente a las consideradas para el sistema de inversión de flujo (Ecs. 7.5 y 7.6).

El volumen adicional que deberá considerarse para que el frente de onda pueda desplazarse, $V_{F}$, será igual a $v_{F}\left(S_{\text {tot,Reg }} / 2\right) t_{\text {ciclo }} / 2$, con lo cual, a partir de la Ec. (8.3), se tiene:

$$
V_{F}=\frac{G_{m, t o t} c_{p G}}{\left(1-\varepsilon_{L}\right)\left(\rho c_{p}\right)_{S}} \frac{t_{c i c l o}}{2}
$$

expresión que resulta idéntica a la Ec. (7.7). 
Con ello, la cota superior para el volumen del regenerador se calcula directamente como $V_{\text {Reg, } \max }=V_{\text {Reg, } \min }+V_{F}$.

Finalmente, con los valores de $V_{\text {Reg,min }}, D_{\text {Reg,min }} \mathrm{y} V_{\text {Reg,max }}$, se puede calcular $D_{\text {Reg,max }}$ de la misma manera indicada en el Capítulo 7 para el sistema de inversión de flujo. Es decir,

$$
D_{\text {Reg, } \max }=D_{\text {Reg, } \min }\left(\frac{V_{R e g, \max }}{V_{R e g, \min }}\left(\frac{\mu_{G}}{\rho_{G}}\right)_{T_{S, \max }-\Delta T_{a d}} /\left(\frac{\mu_{G}}{\rho_{G}}\right)_{T_{m}^{\text {Reg }}}\right)^{1 / 4}
$$

donde se ha introducido la relación $\left(\mu_{G} / \rho_{G}\right)_{T_{S, \max }-\Delta T_{a d}} /\left(\mu_{G} / \rho_{G}\right)_{T_{m}^{\text {Reg }}}$ para tener en cuenta el efecto del mayor nivel térmico en el sistema.

En la Tabla 8.3 se presentan los valores de $V_{\text {Reg,min }}, V_{\text {Reg,max }}, D_{\text {Reg,min }}$ y $D_{\text {Reg,max }}$, predichos mediante la aproximación del intercambiador en contra-corriente y del movimiento del frente de onda. Para obtener tales valores, se consideró una temperatura de alimentación al reactor de $280^{\circ} \mathrm{C}$ y un tiempo de ciclo de 30 s y se adoptó el menor contenido de VOCs.

Tabla 8.3: cotas inferiores y superiores para el volumen y diámetro del regenerador.

\begin{tabular}{|c|c|c|}
\hline$n^{\circ}$ de configuración & $\begin{array}{c}V_{R e g, \min }-V_{R e g, \max } \\
{\left[\mathrm{m}^{3}\right]}\end{array}$ & $\begin{array}{c}D_{R e g, \min }-D_{R e g, \max } \\
{[\mathrm{m}]}\end{array}$ \\
\hline 1 & $3.32-3.54$ & $2.83-3.34$ \\
\hline 2 & $1.72-1.99$ & $2.77-3.33$ \\
\hline 3 & $1.15-1.54$ & $2.61-3.26$ \\
\hline 4 & $1.72-1.95$ & $2.86-3.42$ \\
\hline 5 & $1.15-1.38$ & $2.86-3.47$ \\
\hline
\end{tabular}




\subsubsection{Cotas inferiores y superiores para el reactor}

Considerando que el reactor opera en estado estacionario una vez alcanzado el css, la búsqueda de las cotas para el volumen del mismo se basó en una estimación de las velocidades de reacción máxima (cota inferior de volumen) y minima (cota superior).

Para la cota inferior de volumen, $V_{R, \min }$, se debe tener presente que si la velocidad de reacción en la capa catalítica fuese muy elevada, la reacción quedaría controlada por la transferencia de materia en el canal. En tal caso, el acetaldehído no alcanzaría a acumularse, necesitando solo los balances de materia para los dos componentes restantes, reduciéndose las ecuaciones de balance de materia en el reactor (Ecs. 3.27 y 3.28) a:

$$
\frac{G}{M_{\text {aire }}} \frac{d y_{j, G}}{d z}=-a_{v} k_{m, j} C_{T} y_{j, G} \quad j=1 . .2
$$

Si en adición se consideran los valores asintóticos de los coeficientes de transferencia de materia, $k_{m, j, \infty}$, y las propiedades fisicas constantes (evaluadas a la temperatura media en el reactor: $\left.T_{G}^{0, R}+\Delta T_{a d} / 2\right)$, se pueden integrar las Ecs. (8.6), las cuales resultan independientes entre sí. De esta manera se obtiene una ecuación idéntica a la presentada en el Capítulo 7 para estimar la región necesaria para la reacción en el sistema de inversión de flujo (Ec. 7.2), la cual corresponde a:

$$
\left\langle y_{\text {VOCs }}\right\rangle=y_{1, G}^{0} \exp \left(-\frac{a_{\nu} \rho_{G} k_{m, 1, \infty}}{G_{m, t o t}} V_{R, \min }\right)+y_{2, G}^{0} \exp \left(-\frac{a_{\nu} \rho_{G} k_{m, 2, \infty}}{G_{m, t o t}} V_{R, \min }\right)
$$

donde los valores de $y_{j, G}^{0}$ corresponden al minimo contenido de VOCs.

Por otro lado, para la cota superior de volumen, $V_{R, \max }$, se debe estimar un límite inferior para la velocidad de reacción. A tal fin, se puede suponer que la temperatura del sólido permanece uniforme a lo largo del reactor e igual al va- 
lor promedio entre entrada y salida y que no hay resistencias a la transferencia de energía en el canal (la elevación de temperatura asociada a tal resistencia aumenta la velocidad de reacción). Si bien estas suposiciones simplifican significativamente el sistema de ecuaciones a resolver, el mismo aun será no-lineal. Para sortear este inconveniente se linealizó la velocidad de reacción de cada componente basándose en los balances de materia en la interfase, considerando:

$$
-a_{v, C} k_{m, j, C} C_{T}\left(y_{j, G}-y_{j, S}\right) \approx-a_{v, C} k_{m, j, C} C_{T} \overline{\left[\frac{\left(y_{j, G}-y_{j, S}\right)}{y_{j, G}}\right]} y_{j, G}
$$

donde el término $\overline{\left[\frac{\left(y_{j, G}-y_{j, S}\right)}{y_{j, G}}\right]}$ es un promedio convenientemente elegido para cuantificar la resistencia a la transferencia de materia.

Para tal fin, se resolvieron los balances de materia en la interfase (Ec. 3.28), considerando nuevamente los valores asintóticos para los coeficientes de transferencia de materia, tanto para las condiciones de entrada como para una condición con $y_{j, G} \rightarrow 0$. Con la diferencia relativa obtenida en cada caso, se tomó luego el promedio armónico ${ }^{18}$ :

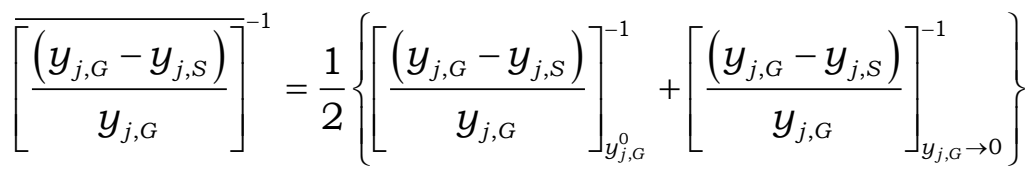

18 Notar que, sin introducir ninguna simplificación, la Ec. (3.27) puede escribirse como:

$$
\int_{y_{j, G}^{\circ}}^{y_{j, G}} \frac{1}{\left[\frac{\left(y_{j, G}^{*}-y_{j, S}\right)}{y_{j, G}^{*}}\right] y_{j, G}^{*}} d y_{j, G}^{*}=-\int_{0}^{V_{R}} \frac{a_{v} k_{m, j} \rho_{G}}{G_{m, t o t}} d V
$$

donde surge naturalmente el promedio armónico para extraer el término $\left[\frac{\left(y_{j, G}-y_{j, S}\right)}{y_{j, G}}\right]$ de la integral. 
Finalmente, teniendo en cuenta las Ecs. (8.8) y (8.9) y considerando que el acetaldehído no se acumula (es decir, el etanol se oxida en forma directa a $\mathrm{CO}_{2}$ ), se pueden integrar los balances de materia, obteniendo una expresión completamente equivalente a la Ec. (8.7), pero debiendo reemplazar los coeficientes de transferencia de materia por el término:

$$
k_{m, j, \infty} \overline{\left[\frac{\left(y_{j, G}-y_{j, S}\right)}{y_{j, G}}\right]}
$$

Con todo lo anterior, se obtuvieron los valores de cotas inferiores y superiores para el volumen del reactor (y los correspondientes valores del diámetro de acuerdo con la Ec. 8.1), para el mínimo contenido de VOCs y una temperatura de alimentación al reactor de $280^{\circ} \mathrm{C}$. Los resultados se muestran en la Tabla 8.4 .

Tabla 8.4: cotas inferiores y superiores para el volumen y diámetro del reactor.

\begin{tabular}{ccc}
\hline $\mathbf{n}^{\mathbf{0}}$ de configuración & $\begin{array}{c}\boldsymbol{V}_{\boldsymbol{R}, \min }-\boldsymbol{V}_{\boldsymbol{R}, \max } \\
{\left[\mathbf{m}^{\mathbf{3}}\right]}\end{array}$ & $\begin{array}{c}\boldsymbol{D}_{\boldsymbol{R}, \min }-\boldsymbol{D}_{R, \max } \\
{[\mathbf{m}]}\end{array}$ \\
\hline $\mathbf{1}$ & $1.01-1.25$ & $1.69-2.08$ \\
\hline $\mathbf{2}$ & $0.51-0.67$ & $1.64-2.04$ \\
\hline $\mathbf{3}$ & $0.34-0.46$ & $1.55-1.94$ \\
\hline $\mathbf{4}$ & $0.51-0.67$ & $1.69-2.12$ \\
\hline $\mathbf{5}$ & $0.34-0.47$ & $1.69-2.14$ \\
\hline
\end{tabular}

\subsection{Justificación de la elección de la temperatura de ali- mentación al reactor}

En los criterios de diseños expuestos en la Sección 8.1 se fijó la temperatura de alimentación al reactor en $280^{\circ} \mathrm{C}$. Como se verá, este valor no solo permite mantener un cierto grado de flexibilidad comparable al de los sistemas 
de inversión de flujo (en términos de la máxima caída de actividad posible), sino que también permite disminuir sustancialmente la masa total de material inerte en el sistema.

El efecto de la caída de actividad fue estudiado en el Capítulo 6, aunque no desde una perspectiva de diseño (se mantuvieron siempre las dimensiones del reactor). Resulta interesante entonces analizar qué ocurre con las dimensiones y material a utilizar al modificar la temperatura de alimentación al reactor en la estrategia de diseño propuesta, registrando en forma simultánea la máxima caída admisible en la actividad del catalizador.

Para realizar este estudio, se consideró únicamente la configuración de celda identificada como 1 en la Tabla 8.2 (es esperable que los resultados sean generales en términos de las tendencias observadas), liberando la condición correspondiente a $T_{G}^{0, R}=280^{\circ} \mathrm{C}$ en los criterios de diseño. Los resultados se muestran en las Figuras 8.1 a 8.3, en donde se ha graficado la masa total de inerte, $W_{I}$ (material del regenerador más el soporte del material catalítico en el reactor), y de catalizador, $W_{C}$, dadas por:

$$
\begin{aligned}
& W_{I}=\rho_{I}\left[V_{R e g}\left(1-\varepsilon_{L, I}\right)+V_{R}\left(1-\varepsilon_{L, C}^{0}\right)\right] \\
& W_{C}=\rho_{C} V_{R}\left(\varepsilon_{L, C}^{0}-\varepsilon_{L, C}\right)
\end{aligned}
$$

donde $\varepsilon_{L, I}$ representa la porosidad del regenerador, $\varepsilon_{L, C}$ la porosidad del reactor y $\varepsilon_{L, C}^{0}$ la porosidad que tendría el mismo sin el depósito catalítico.

Como se observa en las Figuras 8.1 y 8.2, conforme se aumenta la temperatura de alimentación al reactor, las dimensiones del mismo, y consecuentemente la masa de catalizador, disminuyen. No obstante, debido a la resistencia a la transferencia de materia en el canal, tal disminución es cada vez más pequeña. Por otro lado, el volumen y diámetro del regenerador aumentan de forma prácticamente lineal con el aumento de $T_{G}^{0, R}$ (Figura 8.1). Sin embargo, la masa de inerte pasa por un mínimo, debido a que para bajos valores de $T_{G}^{0, R}$ el menor tamaño del regenerador no logra compensar el aumento del tamaño del reactor. Dicho mínimo corresponde a $T_{G}^{0, R} \approx 250{ }^{\circ} \mathrm{C}$. 


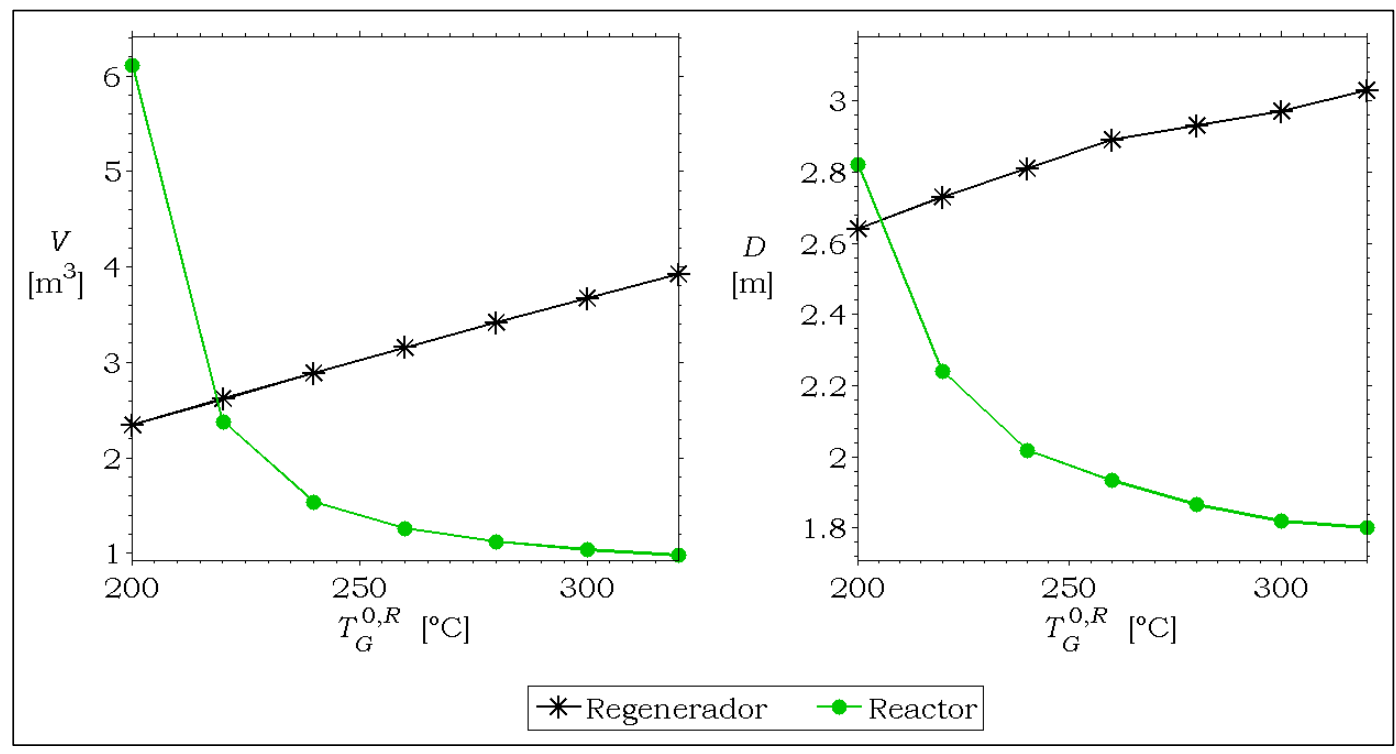

Figura 8.1: efecto de la temperatura de alimentación al reactor sobre el dimensionamiento de ambos equipos (regenerador y reactor), para la configuración 1.

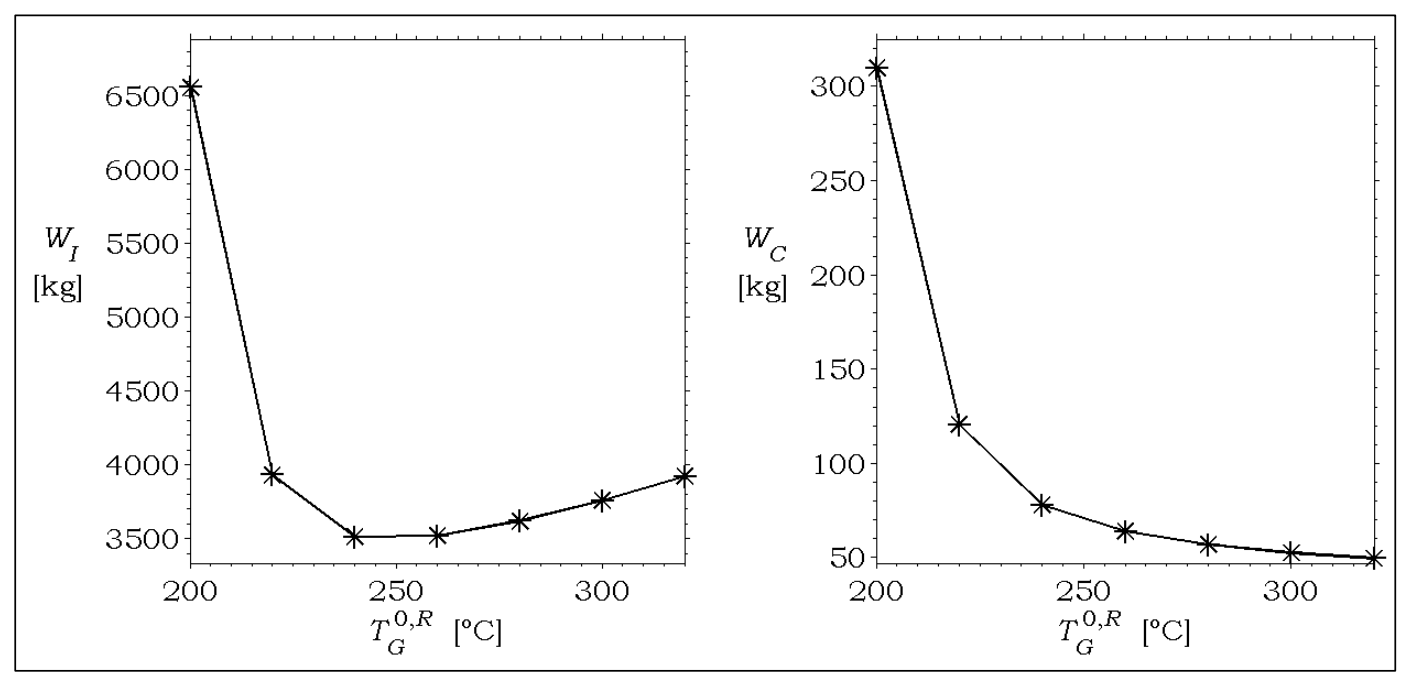

Figura 8.2: efecto de la temperatura de alimentación al reactor sobre la masa total de inerte y catalizador, para la configuración 1.

Por otro lado, en la Figura 8.3 (izquierda) se observa que para la condición con el contenido nominal de VOCs, la temperatura de alimentación al reactor modifica los valores de tiempo de ciclo operativo y crítico en forma aproximadamente lineal para $T_{G}^{0, R} \gtrsim 240^{\circ} \mathrm{C}$. Tal variación es prácticamente idéntica (poseen la misma pendiente), no alterando el rango posible de operación en términos absolutos (lógicamente, si se modifica el rango relativo al tiempo de ciclo operativo). 


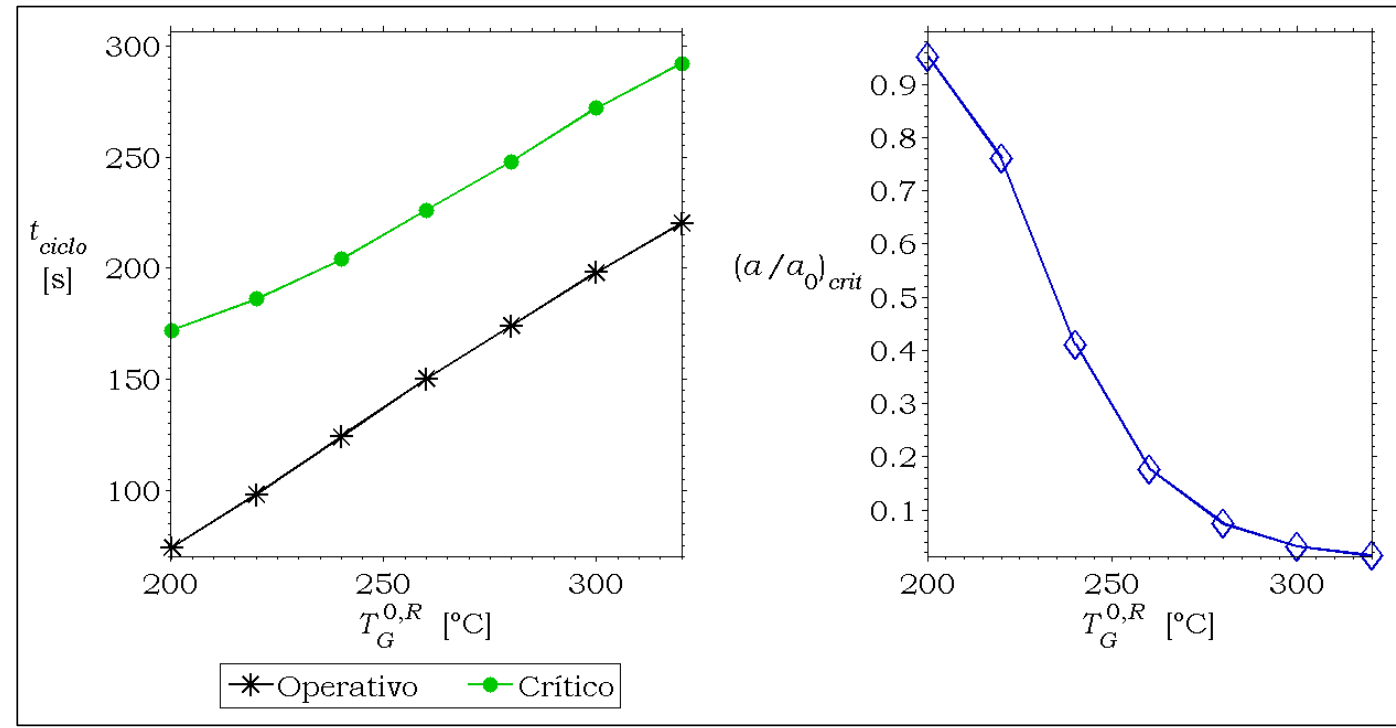

Figura 8.3: efecto de la temperatura de alimentación al reactor sobre los valores de $t_{\text {ciclo }}$ (operativo y crítico) para la condición con el contenido de VOCs nominal y sobre la actividad crítica para la condición con el contenido de VOCs mínimo. Los resultados corresponden a la configuración 1 .

Finalmente, en la Figura 8.3 (derecha) se muestra el valor crítico de la relación entre la actividad del catalizador y la actividad del catalizador fresco, $\left(a / a_{0}\right)_{c r i t}$, para la condición con el menor contenido de VOCs (y consecuentemente con un tiempo de ciclo de $30 \mathrm{~s}$ ). Se puede ver que dicha relación disminuye al aumentar la temperatura de alimentación al reactor, alcanzando valores del orden de un décimo para valores de $T_{G}^{0, R} \gtrsim 270^{\circ} \mathrm{C}$. Cabe resaltar que dicha disminución es significativamente menor a la observada en la Figura 6.4 (ver Sección 6.2), debido a que en tal estudio se habían mantenido las dimensiones del reactor para todos los casos.

El valor de temperatura de alimentación al reactor dado en la estrategia de diseño presentada en la Sección 8.1 se seleccionó teniendo en cuenta todos los elementos volcados en este análisis: disminución de las dimensiones del reactor y de la masa de inerte y catalizador, mejora en los valores de actividad crítica e invariabilidad en el rango (absoluto) de tiempos de ciclo posibles. 


\subsection{Diseños resultantes para cada configuración}

A partir de los criterios de diseño establecidos en la Sección 8.1 y teniendo presente los rangos volcados en las Tablas 8.3 y 8.4 , se buscaron las dimensiones de ambos equipos y el tiempo de ciclo nominal, para las 5 configuraciones dadas en la Tabla 8.2. Los resultados obtenidos se vuelcan en la Tabla 8.5, donde se han añadido los mismos parámetros ya listados en el Capítulo 7 (recordar que $t_{\text {ciclo }}^{*}$ representa el tiempo de ciclo para el cual la emisión global de VOCs es exactamente 9 ppm, correspondiente al valor límite mencionado en el Capítulo 2).

Al igual que para el esquema de inversión de flujo rotativo, se puede observar que al aumentar el número de celdas se logra reducir el volumen de los equipos y la masa de material inerte y catalítico en general. Sin embargo, el uso de las configuraciones 4 y 5 en lugar de las 2 y 3 no posee un impacto significativo en el volumen (el del regenerador resulta ligeramente menor y el del reactor mayor). Por otro lado, la masa total de material catalítico (presumiblemente más costoso que el material inerte) es pequeña en todos los casos. E1 diseño con la configuración 1 es el que presenta el mayor requerimiento de masa de catalizador (un $27 \%$ y un $50 \%$ más que la cantidad requerida por las configuraciones 2 y 5, respectivamente), mientras que en las restantes la diferencia no supera el $20 \%$, por lo que tal variación puede no ser de significancia.

Adicionalmente, para comparar los resultados obtenidos, debe tenerse presente que para el regenerador existe un compromiso entre la longitud y el diámetro. Un diámetro pequeño favorecerá una correcta distribución de flujo (siendo esto último válido también para el reactor). Una longitud pequeña permitirá disminuir el esfuerzo sobre el eje del rotor y los cojinetes respectivos.

Puede verse de la Tabla 8.5 que ninguna de las configuraciones posee un marcado impacto sobre el diámetro de los equipos (la mayor diferencia se da para el reactor entre las configuraciones 3 y 5 , resultando en una sección transversal 20\% superior para la 5). Por otro lado, la longitud del regenerador es en todos los casos satisfactoria, correspondiendo el mayor valor al diseño con la configuración 1 . De hecho, los valores obtenidos se encuentran en el rango de longitudes habitual de los regeneradores comerciales (Regenerative Heat Exchangers For Industry and Process Technology, 2003). 
Tabla 8.5: resumen de resultados obtenidos en el dimensionamiento del esquema regenerador-reactor para diferentes configuraciones de celda, según la propuesta de diseño dada en la Sección 8.1.

\begin{tabular}{|c|c|c|c|c|c|c|}
\hline \multicolumn{2}{|c|}{$n^{\circ}$ de configuración } & \multirow{2}{*}{$\frac{\begin{array}{c}\mathbf{1} \\
\mathbf{( 2 0 0 / 1 2 . 5 )}\end{array}}{3.418}$} & \multirow{2}{*}{$\begin{array}{c}2^{2} \\
1.860 / 7.5)\end{array}$} & \multirow{2}{*}{$\begin{array}{c}3 \\
(600 / 4) \\
1.515\end{array}$} & \multirow{2}{*}{$\begin{array}{c}\mathbf{4} \\
\mathbf{( 4 0 0 / 8 . 8 )} \\
1.832\end{array}$} & \multirow{2}{*}{$\begin{array}{c}5 \\
\mathbf{( 6 0 0 / 7 . 2 )} \\
1.309\end{array}$} \\
\hline & $V_{R e g}\left[\mathrm{~m}^{3}\right]$ & & & & & \\
\hline & $L_{R e g}[\mathbf{m}]$ & 0.490 & 0.273 & 0.225 & 0.254 & 0.175 \\
\hline & $D_{\text {Reg }}[\mathrm{m}]$ & 2.980 & 2.950 & 2.930 & 3.030 & 3.090 \\
\hline & $V_{R}\left[\mathrm{~m}^{3}\right]$ & 1.118 & 0.604 & 0.417 & 0.608 & 0.426 \\
\hline & $L_{R}[\mathrm{~m}]$ & 0.431 & 0.239 & 0.181 & 0.225 & 0.153 \\
\hline & $D_{R}[\mathrm{~m}]$ & 1.817 & 1.794 & 1.711 & 1.854 & 1.882 \\
\hline & $W_{I}[\mathbf{k g}]$ & 3619 & 1680 & 867 & 1935 & 1370 \\
\hline & $w_{c}[\mathbf{k g}]$ & 57 & 45 & 40 & 44 & 38 \\
\hline \multirow{4}{*}{ 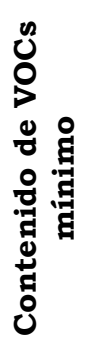 } & $T_{S, \max }\left[{ }^{\circ} \mathbf{C}\right]$ & 306 & 306 & 306 & 306 & 306 \\
\hline & $\left\langle y_{\text {vocs }}\right\rangle[\mathbf{p p m}]$ & 0.995 & 0.995 & 0.998 & 0.996 & 1.002 \\
\hline & $\left|\Delta \boldsymbol{P}_{\text {tot }}\right|$ [mbar] & 40.2 & 40.2 & 39.9 & 40.3 & 40.0 \\
\hline & $\left|\Delta \boldsymbol{P}_{\boldsymbol{R e g}}\right| /\left|\Delta \boldsymbol{P}_{\text {tot }}\right|$ & 0.526 & 0.528 & 0.530 & 0.528 & 0.529 \\
\hline \multirow{8}{*}{ 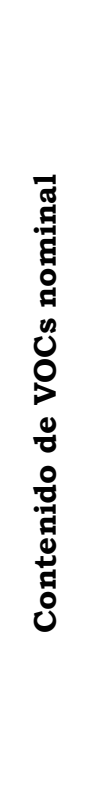 } & $t_{\text {ciclo }}[\mathbf{s}]$ & 174 & 86 & 52 & 97 & 72 \\
\hline & $t_{\text {ciclo,crit }}[\mathbf{s}]$ & 249 & 119 & 67 & 133 & 96 \\
\hline & $\frac{t_{\text {ciclo,crit }}-t_{\text {ciclo }}}{t_{\text {ciclo }}}$ & 0.43 & 0.38 & 0.29 & 0.37 & 0.33 \\
\hline & $t_{c i c l o}^{*}[\mathbf{s}]$ & 247 & 117 & 66 & 132 & 95 \\
\hline & $T_{S, \max }\left[{ }^{\circ} \mathbf{C}\right]$ & 398 & 399 & 400 & 398 & 399 \\
\hline & $\left\langle y_{\text {vocs }}\right\rangle[\mathbf{p p m}]$ & 0.435 & 0.324 & 0.280 & 0.317 & 0.245 \\
\hline & $\left|\Delta \boldsymbol{P}_{\text {tot }}\right|$ [mbar] & 49.8 & 50.0 & 49.5 & 50.0 & 49.7 \\
\hline & $\left|\Delta \boldsymbol{P}_{\boldsymbol{R e g}}\right| /\left|\Delta \boldsymbol{P}_{\text {tot }}\right|$ & 0.514 & 0.514 & 0.515 & 0.514 & 0.514 \\
\hline
\end{tabular}


En adición, también se debe evaluar qué impacto posee el cambio de configuración sobre la estabilidad del sistema. En forma completamente análoga a los sistemas de inversión de flujo, puede verse que el aumento del número de celdas genera una disminución del tiempo de ciclo crítico para la condición con el contenido de VOCs nominal (ver valores para las configuraciones 1, 2 y 3, o 1, 4 y 5). Como fuera mencionado en el Capítulo 7, esto se debe a la disminución de la masa total de los equipos (particularmente para este caso, de la masa de material inerte del regenerador). De hecho, la diferencia entre el tiempo de ciclo seleccionado para la condición de trabajo nominal y el crítico también disminuye (tanto en términos absolutos como relativos ${ }^{19}$ ), indicando que la operación del sistema se torna más sensible (y por lo tanto, con mayor tendencia a la inestabilidad). Nuevamente, el uso de las configuración 5 en lugar de la 3 permite mejorar levemente esta situación en general, mientras que el uso de la configuración 4 en lugar de la 2 permite mejorar ligeramente dicha diferencia en términos absolutos pero la empeora en términos relativos.

\subsubsection{Análisis complementario de los diseños obtenidos}

Para completar el análisis de los diseños alcanzados, se registró la respuesta de cada uno frente a la variación del tiempo de ciclo, para la condición con el contenido de VOCs nominal, cuyos resultados se presentan en la Figura 8.4. Se puede notar que la variación de la temperatura máxima en este esquema resulta sustancialmente más sensible a los cambios en el tiempo de ciclo. En particular, se observa un elevado aumento de la temperatura máxima para tiempos de ciclo menores que el seleccionado en el diseño, llegando a valores prohibitivos (mayores a $600^{\circ} \mathrm{C}$ ).

Cabe recordar que, para la condición de operación nominal, es deseable que los diseños obtenidos ofrezcan un rango de tiempos de ciclo los más amplio posible (en términos relativos al valor seleccionado para tal condición). Esto radica en la necesidad de cubrir el grado de incertidumbre asociado a una etapa de diseño, ya sea en la generalidad del modelo empleado como en la determinación de las expresiones cinéticas y los parámetros involucrados.

\footnotetext{
19 Recordar que la disminución de tal diferencia se podría relacionar con una mayor dificultad en el control del proceso, tal como fuera discutido en el Capítulo 7, Sección 7.1.2.
} 


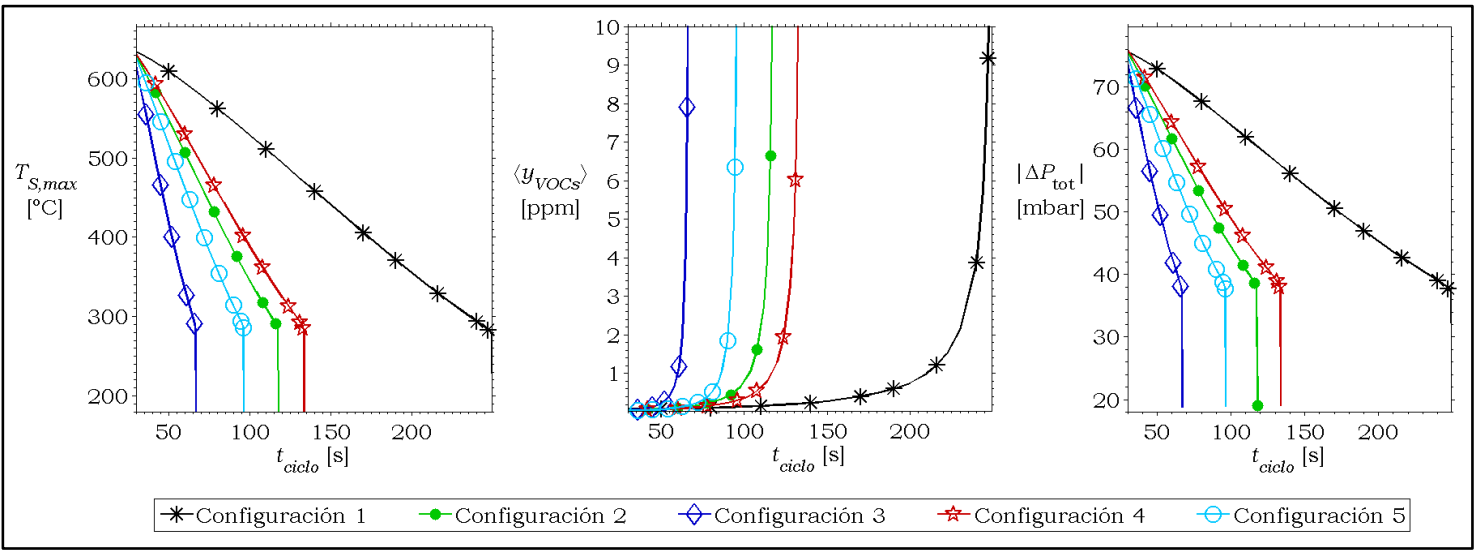

Figura 8.4: respuesta del sistema regenerador-reactor frente a la variación de $t_{\text {ciclo }}$ para el contenido de VOCs nominal y para cada uno de los diseños alcanzados.

En la Figura 8.4 se puede notar la reducción en el tiempo de ciclo crítico (aspecto que también se puede observar en la Tabla 8.5), particularmente para las configuraciones con mayor densidad de celdas (3 y 5).

Teniendo en cuenta que un rango muy estrecho de tiempos de ciclo está asociado a un sistema más sensible a los cambios en alguna de las variables (y por lo tanto tendrá mayor tendencia a la inestabilidad), las configuraciones 3 y 5 quedan desechadas (la diferencia entre $t_{\text {ciclo,crit }}$ y $t_{\text {ciclo }}$, es de 15 y 24 segundos, respectivamente). Por otro lado, el uso de la configuración 4 en lugar de la 2 no presenta un claro beneficio. El volumen del regenerador y del reactor resulta prácticamente igual con una u otra configuración de celda, pero la 4 necesita un $15 \%$ más de material inerte, sin que esto se traduzca en una mayor estabilidad (la diferencia absoluta entre $t_{\text {ciclo,crit }}$ y $t_{\text {ciclo }}$ es de 33 segundos para la configuración 2 y de 36 segundos para la 4, mientras que la relativa es de 0.38 y 0.37 , respectivamente). Por lo tanto, considerando que la configuración 4 fue propuesta para obtener la misma porosidad que en la configuración 1, mientras que la 2 corresponde a una forma estándar comercializada por la empresa Corning (Boger y col., 2004), no se aconseja el uso de la primera.

Entre las dos configuraciones restantes (1 y 2), la elección no parece tan clara. Por un lado, la configuración 1 ofrece el mayor rango de operación, comparable al sistema de inversión de flujo convencional (la diferencia absoluta entre $t_{\text {ciclo,crit }}$ y $t_{\text {ciclo }}$ es de $75 \mathrm{~s}$, mientras que la relativa es de 0.43 ). Por otro lado, la configuración 2 permite disminuir la masa total necesaria a más de la mitad del necesario con la configuración 1, alcanzando valores intermedios a 
los obtenidos con los sistemas de inversión de flujo. Si bien esto se logra a expensas de una disminución equivalente del rango de operación (la diferencia absoluta entre $t_{\text {ciclo,crit }}$ y $t_{\text {ciclo }}$ es de $33 \mathrm{~s}$ ), la diferencia relativa no se ve tan perjudicada, adoptando el valor de 0.38 .

Por lo expuesto anteriormente, se sugiere el uso de la configuración 2 (400/7.5) para el esquema regenerador-reactor.

\subsection{Conclusiones y comentarios finales}

Siguiendo la metodología utilizada para el diseño de los sistemas de inversión de flujo, se identificaron los grados de libertad (7) correspondientes al esquema regenerador-reactor.

En base al análisis realizado en el Capítulo 6, se anularon los dos grados de libertad correspondientes a la fracción de limpieza (fijando $f_{L}=1 \%$ ) y a la fracción de enfriamiento (fijando $f_{E}=49 \%$ ).

A fin de realizar el diseño en condiciones análogas a las utilizadas en el Capítulo 7, se consideró nuevamente el rango de contenido de VOCs dado en la Tabla 8.1. Recordando que la situación más crítica para dimensionamiento de los equipos corresponde al menor contenido de VOCs, se tomó la misma como condición para definir los volúmenes del regenerador y del reactor. Para ello, se debe definir también la temperatura deseada de alimentación al reactor, valor seleccionado en $280^{\circ} \mathrm{C}$. De acuerdo con el análisis realizado en la Sección 8.2, este valor permite reducir significativamente la cantidad de material inerte y mejorar la tolerancia a la caída en la actividad catalítica. En adición, para el dimensionado del regenerador se consideró el tiempo de ciclo mínimo, para el cual se seleccionó el valor de 30 segundos, correspondiente a una velocidad de rotación de $2 \mathrm{rpm}$.

Por otro lado, la definición de la pérdida de carga admisible, la temperatura máxima en el reactor y la relación de diámetros entre el reactor y el regenerador (fijada para que la pérdida de carga en cada equipo sea comparable), permitió especificar el diámetro de los equipos y el tiempo de ciclo en la condición de operación nominal. 
A partir de los criterios de diseño establecidos en la Sección 8.1, se estudiaron las cinco variantes de configuración de celda propuestas en el Capítulo 7, buscando las dimensiones para cada una de ellas. A diferencia de los sistemas de inversión de flujo, se verificó que para este esquema las configuraciones con mayor número de celdas (configuraciones 3 y 5) no resultan adecuadas. Esto se debe principalmente a la reducción del rango de tiempos de ciclos posibles. Por otro lado, la configuración 1 es la que presenta el mayor rango de tiempos de ciclo para la condición nominal (tanto en términos absolutos como relativos), pero requiere prácticamente el doble de material inerte que las alternativas con la configuraciones 2 o 4 y un 29\% más de material catalítico. Las configuraciones 2 y 4 presentan características similares entre sí y aún permiten obtener un rango de tiempos de ciclo adecuado, siendo preferible la primera, por corresponder a una forma estándar actualmente comercializada.

Finalmente, en base a los resultados obtenidos en el presente capítulo, se sugirió el diseño con la configuración 2 para este esquema. 


\section{Capítulo 09}

\section{- Conclusiones finales: comparación. Síntesis de resultados y recomendaciones}

- Análisis de los diseños obtenidos para cada esquema

- Síntesis de resultados alcanzados

- Recomendaciones para futuros trabajos 

En los Capítulos 7 y 8 se propuso una estrategia de diseño para cada esquema, basándose parcialmente en los resultados de los estudios realizados en los Capitulos 5 y 6. A partir de tal estrategia, se consideraron en todos los casos cinco configuraciones de celda diferentes para el monolito, seleccionando luego el diseño más adecuado para cada esquema.

A fin de concluir el presente estudio, en este capítulo se busca comparar cada uno de los esquemas, según los diseños obtenidos, a efectos de sugerir el más adecuado. Debe recordarse que en la práctica no sería recomendable realizar la elección sin haber sometido cada alternativa a una evaluación económica. No obstante, la comparación básica teniendo en cuenta la cantidad de material involucrado (y/o el volumen ocupado) y la flexibilidad del sistema, entre otros, resulta siempre un primer punto de partida en la selección del sistema más adecuado.

Teniendo en cuenta lo discutido en el párrafo anterior, en el presente capítulo se comparan los diseños obtenidos en base a los requerimientos de volumen y masa en cada caso, entre otros. En adición se analiza cómo responde cada uno de los esquemas a variaciones en la actividad del catalizador, para los extremos del rango de contenido de VOCs considerado, a fin de cuantificar su efecto sobre los diseños seleccionados en los Capítulos 7 y 8 .

Finalmente, se presenta una sintesis de los resultados alcanzados en el presente estudio y se dan algunas recomendaciones para trabajos futuros.

\subsection{Análisis de los diseños obtenidos para cada esquema}

En los Capítulos 7 y 8 se seleccionó una configuración de celda para cada uno de los esquemas estudiados, obteniéndose de esa manera los diseños correspondientes, cuyas principales características se resumen en la Tabla 9.1. Cabe señalar que los valores de $t_{\text {ciclo }}, t_{\text {ciclo,crit }} \mathrm{y}$ de la relación $\left(t_{\text {ciclo, crit }}-t_{\text {ciclo }}\right) / t_{\text {ciclo }}$ (tanto en la Tabla 9.1 como en el resto del presente capítulo), corresponden en todos los casos a la condición de operación con el contenido de VOCs nominal.

Si se tiene presente que en la actualidad el diseño de nuevos procesos (o el rediseño de los ya existentes) busca, entre otros aspectos, minimizar el espacio ocupado por los equipos en planta (Stankiewicz y Moulijn, 2000), el esquema 
de inversión de flujo rotativo surge como la mejor alternativa, mientras que el esquema de inversión de flujo convencional resulta el más desfavorecido (ver Tabla 9.1). En cuanto al esquema regenerador-reactor, ambos equipos en conjunto poseen un volumen total intermedio entre los otros dos esquemas.

Tabla 9.1: características principales de los diseños alcanzados para cada esquema.

\begin{tabular}{|c|c|c|c|c|c|}
\hline \multicolumn{2}{|r|}{ Esquema } & $\begin{array}{c}\text { Inversión de flujo } \\
\text { convencional }\end{array}$ & $\begin{array}{c}\text { Inversión de flujo } \\
\text { rotativo }\end{array}$ & \multicolumn{2}{|c|}{ Regenerador-reactor } \\
\hline \multicolumn{2}{|c|}{$\begin{array}{l}\text { configuración de } \\
\text { celda }\end{array}$} & \multirow[t]{2}{*}{$\begin{array}{c}4 \\
(400 / 8.8)\end{array}$} & \multirow[t]{2}{*}{$\begin{array}{c}5 \\
(600 / 7.2)\end{array}$} & \multicolumn{2}{|c|}{$\begin{array}{c}2 \\
(400 / 7.5)\end{array}$} \\
\hline \multirow{4}{*}{ 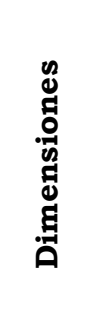 } & & & & Regenerador & reactor \\
\hline & $\boldsymbol{V}\left[\mathbf{m}^{3}\right]$ & 3.385 & 1.538 & 1.866 & 0.604 \\
\hline & $L[\mathrm{~m}]$ & 0.996 & 0.485 & 0.273 & 0.239 \\
\hline & $D[\mathbf{m}]$ & 2.080 & 2.010 & 2.950 & 1.794 \\
\hline & $W_{I}[\mathrm{~kg}]$ & 2606 & 1183 & 1680 & \\
\hline & $W_{c}[\mathbf{k g}]$ & 146 & 68 & 45 & \\
\hline \multirow{3}{*}{ 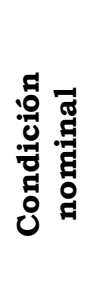 } & $t_{\text {ciclo }}[\mathbf{s}]$ & 270 & 66 & 86 & \\
\hline & $t_{\text {ciclo, } \text {,rit }}[\mathbf{s}]$ & 400 & 152 & 119 & \\
\hline & $\frac{t_{\text {ciclo, } \text {,rit }}-t_{\text {ciclo }}}{t_{\text {ciclo }}}$ & 0.48 & 1.30 & 0.38 & \\
\hline
\end{tabular}

Por otro lado, si se tiene en cuenta la cantidad de material catalítico y/o inerte requerido en cada caso (asociada a un menor costo fijo), el esquema de inversión de flujo convencional queda nuevamente situado en la posición más desfavorable 20 . En cuanto al esquema de inversión de flujo rotativo y el regenerador-reactor, no se puede establecer a priori la conveniencia de uno sobre el otro. Si se compara únicamente la cantidad de material inerte requerido, el esquema de inversión de flujo rotativo quedaría ubicado como el más adecuado (el esquema regenerador-reactor requiere un $42 \%$ más de inerte). Sin embargo, la cantidad de material catalítico necesario para el primero resulta

20 Debe tenerse presente que el resultado enunciado está parcialmente condicionado a la elección del tiempo de ciclo mínimo (240 s para este esquema). Si se permitiera operar con tiempos de ciclos menores (reduciendo la vida útil de la válvula), se podría disminuir el volumen del equipo y la masa de material (tanto inerte como catalítico). 
un $51 \%$ mayor que la correspondiente al esquema regenerador-reactor ${ }^{21}$. Si bien los requerimientos de material inerte son muy superiores a los del catalitico, el mayor costo de este último podría compensar tal situación, o inclusive favorecer al esquema regenerador-reactor, si la diferencia de costo entre el catalizador y el inerte fuese muy marcada (la relación entre el costo del material catalítico e inerte debería ser aproximadamente mayor a 22).

De todas maneras, si bien la cantidad de material sirve para establecer una referencia (especialmente si la diferencia entre una $u$ otra opción es muy elevada), no debería considerarse como único elemento comparativo. Esto se debe a que el costo de producción de los monolitos con mayor número de celdas será presumiblemente más elevado, siendo también necesario contemplar este aspecto. Por lo tanto, el uso de tales configuraciones (por ejemplo, la seleccionada para el esquema de inversión de flujo rotativo) debería estar acompañado de un ahorro en la cantidad de material requerido que justifique su elección, al menos desde el punto de vista económico. En adición, se recuerda que la configuración de celda seleccionada para el esquema regenerador-reactor (identificada previamente como configuración 2), corresponde a una forma estándar comercializada por la empresa Corning (Boger y col., 2004). Por el contrario, tanto la configuración de celda seleccionada para el esquema de inversión de flujo convencional (configuración 4) como para el rotativo (configuración 5), corresponden a alternativas propuestas en el presente estudio, derivadas a partir de las configuraciones correspondientes informadas por Boger y col. (2004), a fin de obtener una porosidad igual a la configuración 1 (ver Sección 7.1 y Tabla 7.2 para una discusión más detallada). Este aspecto representa, en principio, una ventaja para el esquema regenerador-reactor.

Contrariamente a lo discutido en el párrafo anterior, si se observa en la Tabla 9.1 el rango de tiempos de ciclos posibles (tanto absoluto como relativo), el esquema regenerador-reactor resulta más desfavorecido que cualquiera de los esquemas de inversión de flujo, siendo el más prometedor en tal aspecto el es-

\footnotetext{
21 A pesar que la comparación se realiza en base a los diseños sugeridos en cada caso, es interesante notar que si se comparan los diseños resultantes para cada esquema bajo una misma configuración de celda (ver Tablas 7.4, 7.7 y 8.5), el esquema de inversión de flujo convencional requiere siempre mayor cantidad de material inerte y catalitico. Por otro lado, el esquema de inversión de flujo rotativo es el que necesita la menor masa total de inerte, pero la diferencia con el esquema regenerador-reactor no es muy marcada (este último requiere un $26 \%$ más de inerte como máximo), mientras que el esquema regenerador-reactor reduce significativamente la masa de catalizador necesario (al menos en un 44\%).
} 
quema de inversión de flujo rotativo. No obstante, si se recuerda que la conveniencia de que este rango sea relativamente amplio responde a la necesidad de disponer de un margen de flexibilidad para los diseños obtenidos (para cubrir la incertidumbre típica de una etapa de diseño, como fuera discutido en el Capítulo 7) y/o favorecer un adecuado control del proceso, se puede razonar que tales valores serán presumiblemente suficientes en todos los $\operatorname{casos}^{22}$.

Finalmente, debe tenerse en cuenta que en general los sistemas con partes móviles (esquemas de inversión de flujo rotativo y regenerador-reactor -ver Figuras 3.1 y 3.2, respectivamente-) poseen un nivel de complejidad mayor que aquellos de tipo estático (esquema de inversión de flujo convencional). Esto supone un mantenimiento más exhaustivo, lo que se puede traducir en un mayor costo de operación. En adición, el esquema regenerador-reactor necesita un segundo soplador para el abastecimiento del aire utilizado para la limpieza de los canales. No obstante, si bien su presencia en el sistema implica una inversión inicial y un costo de operación adicional, el mismo sería sustancialmente más pequeño que el soplador principal (el caudal de aire utilizado para tal fin es al menos dos órdenes de magnitud inferior al caudal de la corriente a tratar, tal como fue discutido en el Capitulo 6), por lo que ambos factores podrian ser poco relevantes. Por otra parte, el esquema de inversión de flujo rotativo, que también posee una región destinada a la limpieza de los canales, evita la incorporación de otro soplador, pero a costa de una mayor complejidad (comparar los esquemas presentados en las Figuras 3.1 y 3.2).

Debe señalarse que el impacto que puedan tener los elementos discutidos en el párrafo anterior en términos económicos, especialmente la influencia de la mayor complejidad del proceso, es muy dificil de contemplar en forma cualitativa. Por otro lado, su cuantificación requeriría no solo de datos de costos, sino un diseño con mayor grado de detalle (e.g., tipo de sellos en los sistemas rotativos y mantenimiento mínimo de los mismos; mantenimiento y costo de las válvulas del esquema de inversión de flujo convencional; datos de consumo y costo de la energía eléctrica, etc.), aspectos que escapan de los objetivos perseguidos en el presente estudio.

22 Cabe señalar que el menor valor para el esquema regenerador-reactor es intrínseco al esquema en sí, especialmente cuando se observa el rango en términos absolutos (cfr. resultados obtenidos en los Capítulos 7 y 8 en general). Sin embargo, si se desea realizar un análisis de la dinámica del sistema (necesario si se pretendiera estudiar alguna estrategia de control), se debería incluir el comportamiento transitorio del reactor. Por esta razón, la comparación directa entre los rangos obtenidos debe realizarse con precaución. 
A partir de la discusión previa, puede suponerse que el esquema de inversión de flujo convencional será presumiblemente el más costoso de las tres alternativas estudiadas. Sin embargo, con los elementos analizados hasta aquí, este mayor costo no está acompañado de ventajas que pudieran sugerir su elección. De los dos esquemas restantes, el menor volumen y mayor rango de tiempos de ciclo (especialmente en términos relativos al tiempo de ciclo operativo) favorece sin dudas al de inversión de flujo rotativo. Por otro lado, el esquema regenerador-reactor posiblemente resulte más económico (ver discusión sobre la masa requerida de inerte y catalizador en cada caso, junto con la configuración de celda). En adición, esta última alternativa presenta un menor nivel de complejidad en comparación al esquema de inversión de flujo rotativo, tanto en el esquema en sí como en su operación ${ }^{23}$. No obstante, con los elementos analizados hasta aquí, no es posible situar a alguno de estos dos esquemas como el más apto.

Para finalizar, en la siguiente sección se analiza y cuantifica brevemente el desempeño de cada uno de los esquemas frente a la caída en la actividad catalítica. Cabe resaltar que en los Capítulos 5 y 6, tal análisis se realizó en base a los parámetros volcados en cada caso con la única finalidad de ampliar el conocimiento sobre el funcionamiento de los esquemas. Por el contrario, en la siguiente sección se pretende cuantificar tal efecto para los diseños seleccionados en los Capítulos 7 y 8 , a fin de obtener valores de la relación $\left(a / a_{0}\right)_{\text {crit }}$ más representativos, completando los aspectos discutidos en los capítulos previos y disponiendo así de un último elemento comparativo.

\subsubsection{Comportamiento frente a la disminución de la activi- dad catalitica}

Como fuera discutido en los Capítulos 5 y 6, la única alternativa en estos sistemas frente a la caída en la actividad catalítica consiste en incrementar la temperatura de operación. De ser necesario, esto se puede lograr disminuyen-

\footnotetext{
23 Recordar que mientras el regenerador opere en un estado estacionario cíclico (css), el reactor operará en un estado estacionario "puro". Tal como fuera discutido en el Capítulo 6, conceptualmente el sistema se comportará de la misma manera que un esquema autotérmico convencional (i.e., con un intercambiador de superficie) y la modificación del tiempo de ciclo (velocidad de rotación del regenerador), permitirá variar la capacidad de intercambio de energía.
} 
do el tiempo de ciclo, lo que aumenta la capacidad de intercambio de energía en cualquiera de los tres esquemas estudiados.

Se puede razonar fácilmente que la condición más crítica para poder sostener la operación del sistema frente a una caída en la actividad catalítica corresponde a la condición de trabajo con el mínimo contenido de VOCs. En tal condición, si se admite que el tiempo de ciclo no se podrá disminuir por debajo del mínimo establecido en los Capítulos 7 y 8 (240 s para el esquema de inversión de flujo convencional y 30 s para los otros dos), el sistema queda completamente restringido. Por esta razón, y recordando que los sistemas de inversión de flujo tienden a elevar naturalmente la temperatura mientras que el sistema regenerador-reactor no posee tal capacidad, se busca cuantificar la máxima caída admisible para cada uno de los diseños seleccionados.

Para las condiciones mencionadas, se buscó el valor umbral de la actividad catalítica respecto de la actividad del catalizador fresco, $\left(a / a_{0}\right)_{c r i t}$, para el cual un ulterior descenso de la misma no permitiría mantener el css encendido sin introducir alguna modificación en la operación. En adición, también se registró el valor de la relación $a / a_{0}$ para el cual la emisión de VOCs alcanza exactamente los $9 \mathrm{ppm}$ (identificada como $a^{*} / a_{0}$ ), correspondiente a la cota de emisión global mencionada en el Capítulo 2. Los valores encontrados se vuelcan en la Tabla 9.2, donde se observa que cualquiera de los esquemas analizados permite mantener la operación en las condiciones analizadas, con niveles de actividad catalítica tan bajos como la octava parte de la correspondiente al catalizador fresco (a fin de evitar una emisión de VOCs por encima de los $9 \mathrm{ppm})$. Cabe aclarar que la diferencia en los valores de actividad crítica entre el esquema de inversión de flujo convencional y el rotativo se debe a la menor masa de material catalítico en este último, resultado de la conjunción de dos factores: el menor tiempo de ciclo mínimo y la mayor proporción de la longitud de la región inerte.

Adoptando el valor de $a / a_{0}=1 / 5$ como nivel de referencia para una importante caída de actividad catalítica, se registró la respuesta de los tres esquemas para la condición de trabajo con el contenido de VOCs nominal a fin de verificar que en los sistemas de inversión de flujo no haya una elevación de temperatura muy brusca. No obstante, cabe recordar que el valor de temperatura máxima fijado en la etapa de diseño fue elegido de forma tal que se pueda 
disponer de un cierto margen que permitiera elevar la temperatura de operación sin mayores inconvenientes (por ejemplo, en el orden de los $50{ }^{\circ} \mathrm{C}$ ).

Tabla 9.2: valores de la mínima actividad catalitica tolerable en cada sistema, según los diseños obtenidos para la condición de trabajo con el contenido de VOCs mínimo. $\left(a / a_{0}\right)_{c r i t}$ representa la actividad crítica, mientras que $a^{*} / a_{0}$ representa el valor para el cual la emisión de VOCs alcanza los 9 ppm.

\begin{tabular}{cccc}
\hline Sistema & $\begin{array}{c}\text { Inversión de flujo } \\
\text { convencional } \\
\left(\boldsymbol{t}_{\text {ciclo }}=\mathbf{2 4 0} \mathbf{s}\right)\end{array}$ & $\begin{array}{c}\text { Inversión de flujo } \\
\text { rotativo } \\
\left(\boldsymbol{t}_{\text {ciclo }}=\mathbf{3 0} \mathbf{s}\right)\end{array}$ & $\begin{array}{c}\text { Regenerador-reactor } \\
\left(\boldsymbol{t}_{\text {ciclo }}=\mathbf{3 0} \mathbf{~ s}\right)\end{array}$ \\
\hline$\left(\boldsymbol{a} / \boldsymbol{a}_{0}\right)_{\text {crit }}$ & 0.040 & 0.081 & 0.101 \\
\hline $\boldsymbol{a}^{*} / \boldsymbol{a}_{0}$ & 0.057 & 0.116 & 0.111 \\
\hline
\end{tabular}

Los valores obtenidos se muestran en la Tabla 9.3, de donde se puede corroborar que efectivamente la operación en la condición de trabajo nominal no presenta mayores inconvenientes para ninguno de los esquemas analizados, ya sea desde el punto de vista de la emisión de VOCs o de la temperatura máxima en el lecho.

Tabla 9.3: emisión promedio de VOCs y temperatura máxima en cada sistema, según los diseños obtenidos, para la operación con el contenido de VOCs nominal, habiendo reducido la actividad catalítica a $1 / 5$ del valor correspondiente al catalizador fresco. Los tiempos de ciclo para cada esquema corresponden a los informados en la Tabla 9.1.

\begin{tabular}{cccc}
\hline Sistema & $\begin{array}{c}\text { Inversión de flujo } \\
\text { convencional }\end{array}$ & $\begin{array}{c}\text { Inversión de flujo } \\
\text { rotativo }\end{array}$ & Regenerador-reactor \\
\hline$\left\langle\boldsymbol{y}_{\text {vocs }}\right\rangle[\mathbf{p p m}]$ & 0.732 & 0.016 & 0.431 \\
\hline $\boldsymbol{T}_{\text {s, } \max }\left[{ }^{\circ} \mathbf{C}\right]$ & 419 & 412 & 399 \\
\hline
\end{tabular}

A partir de la discusión realizada en los párrafos anteriores, se puede concluir que los tres esquemas analizados en el presente estudio son aptos para el tratamiento de la corriente de VOCs considerada. No obstante, el esquema de inversión de flujo convencional no pareciera ser una alternativa competitiva, frente a los otros dos esquemas analizados. Por esta razón, no se recomienda tal esquema. Por otro lado, tanto el esquema de inversión de flujo rotativo co- 
mo el esquema regenerador-reactor se posicionan como alternativas prometedoras. La decisión final para seleccionar uno u otro esquema debería basarse en estimaciones de costos y/o detalles de tipo constructivos, no contemplados en el presente estudio, que arrojaren nuevos elementos técnicos para la comparación.

\subsection{Sintesis de resultados alcanzados}

En el presente estudio se planteó como objetivo general el análisis de diferentes esquemas de oxidación catalítica para la eliminación de compuestos orgánicos volátiles (VOCs). En particular, se consideró una corriente de aire conteniendo acetato de etilo y etanol, proveniente de la ventilación del recinto de trabajo de plantas de impresión de envases.

Como es frecuente en estos casos, la corriente a tratar se caracteriza por un elevado caudal $\left(9.86 \mathrm{Nm}^{3} / \mathrm{s}\right)$, una temperatura cercana a la ambiente $\left(50^{\circ} \mathrm{C}\right) \mathrm{y}$ un bajo contenido de VOCs (que, para el contenido nominal, se puede traducir en un salto térmico adiabático, $\Delta T_{a d}$, de tan solo $53^{\circ} \mathrm{C}$ ). Por otro lado, si bien existe una búsqueda continua de nuevos materiales cataliticos que permitan llevar a cabo la oxidación a bajas temperaturas, el nivel térmico requerido suele ser de al menos unos centenares de grados (en el caso particular estudiado resulta $T_{i g n} \approx 200^{\circ} \mathrm{C}$ ). Teniendo en cuenta la conjunción de los factores mencionados, resulta inmediato que la modalidad empleada para elevar la temperatura de la corriente será de alta significancia económica. Surge entonces como alternativa básica el aprovechamiento de la energía liberada por la reacción para precalentar la corriente a tratar, formando así un esquema autotérmico. No obstante, la baja fuerza impulsora para el intercambio de energía, junto con la baja capacidad de intercambio intrínseca de las corrientes gaseosas, impone un desafio adicional. Estos aspectos, sumados a la intención de dar continuidad a acciones previas del grupo de investigación, motivó el estudio de tres esquemas autotérmicos de combustión catalítica:

* reactor de inversión de flujo con inversión mediante válvulas (esquema de inversión de flujo convencional); 
* reactor de inversión de flujo con inversión mediante la rotación sobre un eje (esquema de inversión de flujo rotativo);

* reactor catalítico estacionario con precalentamiento de la corriente de aire mediante un intercambiador de calor rotativo (esquema regenerador-reactor).

Todos estos esquemas poseen una característica común: el intercambio de energía se realiza en forma regenerativa en lugar de recuperativa. Una de las principales ventajas de este tipo de intercambio es que los equipos utilizados para tal fin poseen una relación de área de intercambio frente al volumen total muy superior a los intercambiadores de superficie.

Adicionalmente, teniendo en cuenta que las reacciones involucradas son muy rápidas y que es deseable operar con una baja pérdida de carga (al menos inferior a 0.1 bar, de forma que se pueda aportar la sobrepresión necesaria por medio de un soplador estándar), se consideraron en todos los casos lechos estructurados (monolitos) de canales cuadrados, tanto en las regiones inertes como catalíticas. Las regiones inertes de los equipos se consideraron constituidas enteramente por cordierita no porosa, mientras que para las cataliticas, se consideró que el catalizador (óxido de $\mathrm{Mn}$ y $\mathrm{Cu}$, identificado como $\mathrm{Mn}_{9} \mathrm{Cu}_{1}$ ) se encontraba depositado en forma uniforme sobre una matriz de cordierita no porosa, en un espesor de $10 \mu \mathrm{m}$.

Finalmente, para cada uno de los esquemas mencionados se analizaron cinco alternativas para la configuración de la celda de los monolitos, variando la densidad de celda y la sección transversal libre. En cada caso, se buscó cubrir un rango de contenido de VOCs en la corriente a tratar, correspondiendo los extremos mínimo y máximo considerados a la mitad y a la totalidad del contenido nominal respectivamente (con un $\Delta T_{a d}$ de 26.6 y $53^{\circ} \mathrm{C}$ ).

\subsubsection{Simulación de la operación de cada sistema}

Como es propio de los sistemas de intercambio regenerativo, los esquemas estudiados trabajan naturalmente en un estado transitorio, pero cíclicamente estacionario (css). En el caso de los dos esquemas de inversión de flujo, esto se verifica en todo el equipo. Para el esquema regenerador-reactor, solo el re- 
generador trabaja en tal condición, mientras que el reactor opera en un estado estacionario "puro".

Como se discutió en el Capítulo 3, la simulación de cualquiera de los esquemas planteados para encontrar el css requiere de la resolución de las ecuaciones de balances de energía y materia en cada semiciclo, incluyendo los términos de acumulación, hasta que los perfiles de las variables en un ciclo dado sean iguales a los del ciclo anterior. Para ello se consideró un modelo unidimensional heterogéneo implementando dos tipos de algoritmos para su resolución, discutidos en el Capítulo 4. El primero de ellos, que se identificó como "algoritmo interno" (haciendo alusión al cálculo de cada semiciclo), se basó en discretizar la variable espacial y aproximar las derivadas correspondientes por expresiones de tipo diferencias finitas atrasadas, avanzando en la coordenada axial punto a punto e integrando en el tiempo las ecuaciones resultantes localmente. La implementación de este algoritmo en el simulador permite utilizar aproximaciones de orden 1 hasta 5, habiendo encontrado que la más eficiente en términos de precisión y tiempo de cálculo requerido es la correspondiente al orden 2. El segundo algoritmo, que se identificó como "algoritmo externo" (haciendo alusión a la búsqueda del css, supuesto se disponga de la solución de cada ciclo), se basó en el tratamiento de la búsqueda del css como una raíz (cada ciclo simulado se visualiza como una iteración), utilizando la información de iteraciones precedentes para acelerar el proceso de búsqueda. Este algoritmo permitió reducir el tiempo de cálculo requerido para encontrar el css en el esquema de inversión de flujo convencional en 4 veces. No obstante, para evitar inconvenientes cuando el css se encuentra en la cercanía del límite entre los estados encendido y apagado se requiere que los errores entre iteraciones siempre disminuyan (caso contrario se abandona el proceso de aceleración y se continúa por la vía directa).

La combinación de ambos métodos resultó sumamente eficiente y se puede extender a cualquier problema de similares características (e.g., simulación de ciclos de adsorción). En particular, representa una alternativa a los métodos utilizados por otros autores donde se trabaja también discretizando la variable espacial pero se resuelve todo el dominio en simultáneo (e.g., Marín y col., 2010), lo cual puede ser muy exigente a nivel computacional a medida que el número de componentes y/o la complejidad de las expresiones cinéticas aumenta. De todas maneras, cabe aclarar que la resolución secuencial de cada 
punto del dominio espacial, aproximando las derivadas espaciales mediante diferencias finitas atrasadas, no podrá llevarse a cabo si es necesario incluir explícitamente algún término de tipo dispersivo. En tal caso, se debe resolver cada semiciclo en todo el dominio espacial en simultáneo. Sin embargo, se podrá mantener el algoritmo de aceleración.

\subsubsection{Sistemas de inversión de flujo}

La operación con inversión periódica del sentido de circulación permite llevar a cabo la combustión catalítica al nivel térmico requerido sin necesidad de incorporar un equipo de intercambio adicional, pudiendo reemplazar el material catalítico en los extremos del equipo por material inerte únicamente. La alternativa convencional para realizar tal inversión consiste en el uso de una válvula de 4 vías (o un conjunto de válvulas simples) que cambia regularmente su posición direccionando el flujo en un sentido o en otro. Si bien esta alternativa es la más sencilla (y la que ha sido más estudiada en la bibliografia correspondiente), presenta dos desventajas relacionada con los tiempos de ciclo bajos. El primero de ello es el desgaste de las válvulas de inversión, que en promedio poseen una vida útil de unos 500000 movimientos (de acuerdo a $\mathrm{Ba}$ rresi y col., 2007). El segundo problema corresponde a la emisión de los VOCs que se sitúan en la región de entrada al equipo y que son expulsados ( $\sin$ tratar) al invertir el sentido de circulación. Este efecto está siempre presente en el esquema convencional pero su peso en la emisión global promedio depende del tiempo de ciclo empleado, siendo tanto más relevante cuanto menor es el mismo.

Como se corroboró en el Capítulo 5, el esquema de inversión de flujo rotativo, en donde la inversión de la corriente se realiza a través de la propia rotación del equipo, permite eliminar ambos problemas mencionados en el párrafo anterior, al eliminar las válvulas y e incluir una pequeña fracción de la sección total de flujo destinada al barrido de los VOCs residuales previo a la inversión del sentido de circulación. La principal desventaja de esta alternativa es su mayor complejidad frente al esquema convencional.

Cabe señalar que mientras las dimensiones y condiciones de operación sean iguales en ambos esquemas y la fracción de limpieza sea pequeña, el compor- 
tamiento térmico de los mismos resulta prácticamente idéntico, mientras que la emisión de VOCs presenta importantes diferencias a tiempos de ciclo bajos y moderados (siendo muy marcadas para valores de tiempos de ciclos menores a los 100 segundos para el caso estudiado), como consecuencia de la emisión de VOCs residuales al invertir el sentido de circulación en el esquema convencional (Capítulo 5). Más aún, si no existiera una fracción destinada a la limpieza, el comportamiento de uno u otro esquema sería idéntico.

Por otro lado, hay dos conceptos que resultan fundamentales para comprender el funcionamiento de estos esquemas y que representan situaciones límite. El primero corresponde a la similitud entre el sistema de inversión de flujo, cuando el mismo se opera a muy bajos tiempos de ciclo, y un reactor hipotético en contra-corriente. El segundo es el concepto de la formación y movimiento del frente de onda térmico.

En particular, el concepto del reactor en contra-corriente resulta muy útil básicamente por dos aspectos. En primer lugar, como fuera analizado en el Capítulo 5, permite explicar cualitativamente gran parte del comportamiento observado en los sistemas de inversión de flujo frente a la variación de diferentes parámetros (especialmente el aumento de la temperatura máxima al disminuir la actividad catalítica). Esto permite concluir que los principios básicos que determinan la forma en que responde el sistema son propios del funcionamiento dual de la región catalíticamente activa (como catalizador propiamente dicho y como región de intercambio de energía en forma regenerativa) y no del comportamiento transitorio del sistema. El segundo aspecto de interés está relacionado con la capacidad de retener la energía liberada por la reacción. A medida que disminuye el tiempo de ciclo en el esquema de inversión de flujo, dicha capacidad aumenta como consecuencia del menor recorrido del frente de onda, ampliándose la región del equipo donde la temperatura permanece prácticamente uniforme e igual al valor máximo. Para tiempos de ciclo muy pequeños, el recorrido del frente de onda se vuelve prácticamente despreciable y el sistema se comporta como un reactor en contra-corriente. En tal situación, la capacidad para retener la energía liberada por la reacción es máxima. Este razonamiento permitió establecer una cota mínima de volumen necesario en la etapa de diseño para ambos esquemas, convencional y rotativo (Capítulo 7 ). 
Por otro lado, a partir de la expresión que surge de considerar un lecho de longitud infinita por donde circula la corriente a tratar en un único sentido (Matros y Bunimovich, 1996), se puede obtener una cota máxima de la velocidad del frente de onda. Dicha aproximación, en conjunto con la cota mínima de volumen, permitió establecer un límite superior para el volumen necesario del equipo para ambos esquemas, definiendo de esta manera un rango de búsqueda para hallar las dimensiones de los equipos (Capítulo 7).

En adición, en el Capítulo 5 se estudió cómo responde el sistema frente a la variación de diferentes parámetros (tiempo de ciclo, volumen total, proporción de la longitud de la región de inerte en el equipo frente a la longitud total, contenido de VOCs, caída en la actividad catalítica), a fin de ampliar el conocimiento sobre su funcionamiento y disponer así de más conceptos para proponer una estrategia de diseño adecuada. La respuesta a variaciones en el tiempo de ciclo resulta de especial interés debido a que es la única variable operativa cuando el equipo está instalado. Por otro lado, del análisis realizado se pudo corroborar que la fracción de la región inerte en el equipo es una de las variables qué más influye en el comportamiento del sistema. El mayor efecto se da para fracciones superiores al 50\%, aumentando significativamente la temperatura máxima en el sólido a medida que dicha fracción aumenta, así como su sensibilidad con el resto de los parámetros. Por el contrario, para valores pequeños (por ejemplo, menores a 25\%) no se observan cambios apreciables respecto de la operación del equipo cuando todo el lecho es catalítico.

Otro aspecto distintivo del sistema es la respuesta frente a la desactivación del catalizador. Al disminuir la actividad catalítica, la emisión de VOCs aumenta (tal como es esperable), pero la temperatura máxima en el lecho se eleva en lugar de disminuir. Esta respuesta atípica se debe justamente al comportamiento dual del catalizador, como se deduce del estudio del mismo efecto a partir de la aproximación del reactor en contra-corriente y/o de las simplificaciones realizadas en base a tal aproximación (Ec. 5.5). Como consecuencia de la menor actividad, la longitud efectiva de precalentamiento aumenta hasta que la temperatura es suficiente para que la reacción proceda nuevamente a velocidades apreciables. No obstante, disminuye la longitud disponible para la reacción aumentando la emisión de VOCs. 


\subsubsection{Diseño de los sistemas de inversión de flujo}

En el Capítulo 7 se abordó el dimensionamiento de los dos esquemas de inversión de flujo propuestos en el presente estudio. Para ello, se tuvo en cuenta que los sistemas de inversión de flujo resultan más eficientes (en términos de la capacidad de retener la energía liberada por la reacción) cuanto más bajo sea el tiempo de ciclo. Por lo tanto, el uso de tiempos de ciclo pequeños conduce a equipos más compactos. En el límite, se requeriría el mismo volumen que el predicho por la aproximación del reactor en contra-corriente. No obstante, el mínimo tiempo de ciclo que es factible emplear en el esquema convencional está limitado por dos factores: la emisión de los VOCs residuales y la vida útil de la válvula. Por esta razón, se consideró para el diseño del equipo un tiempo de ciclo minimo de 240 segundos. Con ello se logra extender la vida útil de las válvulas en al menos dos años y se evita que la emisión residual de VOCs posea un elevado peso en la emisión global promedio. Sin embargo, tal tiempo de ciclo es lo suficientemente elevado como para que la onda térmica formada en estos sistemas se desplace considerablemente. Lo anterior redunda en un volumen de equipo significativamente mayor que el mínimo requerido (calculado con el reactor en contra-corriente). Esto limita la proporción de inerte que se puede utilizar, a fin de evitar que la temperatura máxima en el lecho alcance valores muy elevados cuando se opera en la condición nominal y, por lo tanto, se necesite trabajar con tiempos de ciclos muy cercanos al crítico. Por tal razón, se limitó la fracción de inerte en el esquema de inversión de flujo convencional al 40\%.

Por otro lado, para el esquema de inversión de flujo rotativo, el tiempo de ciclo mínimo queda limitado por la máxima velocidad de rotación admisible, la cual se fijó en $2 \mathrm{rpm}$ (equivalente a un tiempo de ciclo de 30 segundos). Con el uso de dicho tiempo de ciclo mínimo, el volumen necesario no difiere significativamente del mínimo, resultando diseños más compactos. Ello permite el uso de una fracción de inerte mayor, por lo cual se fijó el valor de la misma en un 50\%. En adición, en este esquema aparece un nuevo grado de libertad dado por la fracción de la sección transversal del equipo que es destinada para la limpieza de los canales previo a la inversión del sentido de circulación (fracción de limpieza). En base al estudio del impacto que posee esta variable sobre 
la operación del sistema, se fijó el valor de la misma en un $2 \%$ para todos los casos.

Las restantes condiciones de diseño fueron generales a ambos esquemas de inversión: para la condición de operación con el mínimo contenido de VOCs se fijó el tiempo de ciclo en su menor valor y se exigió una emisión promedio de VOCs de $1 \mathrm{ppm}$. Para la condición de operación con el contenido de VOCs nominal se exigió que la pérdida de carga sea de 50 mbar y, de ser posible, que simultáneamente la temperatura máxima en el lecho sea de $400{ }^{\circ} \mathrm{C}$. Con ello, se determinó el volumen y diámetro del equipo y el tiempo de ciclo para la condición de operación nominal (igual al mínimo si la temperatura máxima en el lecho para tal valor resultase menor a $400{ }^{\circ} \mathrm{C}$ ).

A partir de los criterios de diseño mencionados en los párrafos anteriores, se obtuvieron las dimensiones del equipo para cada una de las configuraciones de celda estudiadas en cada esquema. Se corroboró a partir de ello que la configuración de celda identificada como 3 (600/4) no resulta adecuada para ninguno de los dos esquemas, principalmente debido a la elevada porosidad que presenta la misma. Del análisis de resultados obtenidos (centrándose principalmente en los requerimientos de masa de inerte y catalizador, así como en la sensibilidad de la respuesta frente a la variación del tiempo de ciclo y el rango de tiempos de ciclos relativos al valor seleccionado para la condición nominal), se sugirió el diseño con la configuración 4 (400/8.8) para el esquema de inversión de flujo convencional y con la configuración 5 (600/7.2) para el esquema de inversión de flujo rotativo.

\subsubsection{Sistema regenerador-reactor}

El esquema regenerador-reactor representa una alternativa básica al esquema autotérmico convencional, formado por un intercambiador de superficie (presumiblemente de tipo compacto) y un reactor adiabático. La diferencia se da en el tipo de intercambiador, reemplazándolo por un regenerador rotativo. La principal ventaja del regenerador es que posee valores de área de intercambio por unidad de volumen, $a_{v}$, muy superiores a los intercambiadores de superficie (inclusive a los conocidos como compactos, en los cuales la relación $a_{v}$ alcanza típicamente valores entre 200 a $800 \mathrm{~m}^{2} / \mathrm{m}^{3}$ 
-Hesselgreaves, 2001-), por lo cual resulta un equipo significativamente más compacto (una excepción a lo anterior pueden ser los intercambiadores conocidos como "intercambiadores de calor de circuito impreso", del que se han fabricado equipos con valores de $a_{v}$ que superan los $2500 \mathrm{~m}^{2} / \mathrm{m}^{3}$ Hesselgreaves, 2001-). Como contraparte, el equipo resulta más complejo y, para la presente aplicación, requiere de un soplador adicional para la circulación del aire utilizado para la limpieza de los canales previos a la inversión del sentido de circulación.

Al igual que en los esquemas de inversión de flujo, el tiempo de ciclo es la única variable operativa disponible en el sistema una vez instalados los equipos. Cabe señalar que, en términos de la capacidad de transferencia del regenerador, el efecto de dicha variable es inmediato: a medida que se aumenta el tiempo de ciclo el frente de onda penetra en mayor medida en el regenerador, disminuyendo la capacidad de transferencia global del mismo y, por ende, la temperatura de entrada al reactor ${ }^{24}$. Esto resultó particularmente evidente en el análisis del efecto de la caída de la actividad catalítica realizado en el Capítulo 6, donde se observó una fuerte influencia de la temperatura de alimentación al reactor sobre la actividad crítica.

Otro aspecto analizado en el Capítulo 6 para este esquema fue el efecto que posee la presencia de la fracción de limpieza (necesaria para evitar la emisión residual de VOCs, lo cual resulta propio de cualquier esquema regenerativo donde se invierta el sentido de circulación de la corriente). De dicho análisis se concluyó que para lograr el barrido de los VOCs residuales es suficiente la presencia de una fracción de limpieza del 1\% de la sección transversal total. En adición, se sugirió que la suma de la fracción de limpieza y la fracción correspondiente a la región de enfriamiento del sólido sea de la mitad del total de la sección transversal, aspecto que se justificó brevemente mediante la igualdad de las velocidades másicas en cada sección.

Finalmente, en el Apéndice 6.1 se realizó un análisis cualitativo de los esquemas autotérmicos para el tratamiento de VOCs, a partir de la formulación simplificada para el regenerador, correspondiente a la operación con tiempos de ciclos muy pequeños (recordar que se comporta como un intercambiador

\footnotetext{
${ }^{24}$ La variación de la temperatura (de alimentación al reactor y, consecuentemente, de la máxima) con el tiempo de ciclo resultó significativamente más pronunciada que en los sistemas de inversión de flujo.
} 
hipotético en contra-corriente). A partir de ese estudio se corroboró que los valores extremos de conversión de VOCs de los estados apagado y encendido, típicos en estos sistemas, está íntimamente ligado a la presencia de reacciones con una elevada energía de activación y con el requerimiento de una capacidad de intercambio alta.

\subsubsection{Diseño del sistema regenerador-reactor}

En el Capítulo 8 se abordó el diseño del esquema regenerador-reactor, para lo cual se tuvo en cuenta que, al igual que los sistemas de inversión de flujo, el regenerador resulta más eficiente cuanto más bajo sea el tiempo de ciclo (lo que se puede traducir en una mayor capacidad de intercambio). Por lo tanto, resulta deseable el uso de tiempos de ciclo pequeños para reducir el tamaño del regenerador (en el límite se requeriría el mismo volumen que el predicho por la aproximación del intercambiador en contra-corriente). Tomando como referencia la velocidad de rotación típica de los regeneradores comerciales, se adoptó el valor de 30 segundos como tiempo de ciclo mínimo ( $2 \mathrm{rpm}$ ). Por otro lado, en base al análisis realizado sobre el efecto de la fracción de limpieza, $f_{L}$ , y de la fracción de la región de enfriamiento del sólido, $f_{E}$, se fijaron para cada una los valores de $1 \%$ y $49 \%$, respectivamente.

Otro aspecto de alta relevancia para el dimensionamiento de este esquema es el valor deseado para la temperatura de alimentación al reactor, $T_{G}^{0, R}$. En primer lugar, se debe tener en cuenta que la condición de diseño más crítica (en términos del volumen requerido para cada equipo) corresponde a la operación con el mínimo contenido de VOCs. En adición, al aumentar la temperatura de alimentación al reactor, el volumen requerido del mismo disminuye como consecuencia de la mayor velocidad de reacción (limitada no obstante por la velocidad de transferencia de materia entre la fase gaseosa y la superficie del sólido) y la actividad crítica resulta más baja. Por el contrario, el volumen del regenerador aumenta conforme aumenta la temperatura de alimentación al reactor. Existe entonces un compromiso entre el volumen (y flexibilidad) del reactor y el volumen del regenerador, que puede traducirse en el requerimiento de masa total de inerte. Esto se estudió para la configuración de celda identificada como 1 , evidenciando un minimo en la masa total de 
inerte a $T_{G}^{0, R} \approx 250^{\circ} \mathrm{C}$. Finalmente, considerando que el aumento de la masa total de inerte no es significativo para $T_{G}^{0, R}=280^{\circ} \mathrm{C}$, mientras que se logra disminuir más el valor de actividad crítica y la masa total de material catalítico, se seleccionó dicho valor como condición de diseño.

Teniendo en cuenta el análisis realizado sobre la temperatura de alimentación al reactor, el volumen de ambos equipos se ajustó de forma tal que en la operación con el mínimo contenido de VOCs, operando con el menor tiempo de ciclo, se obtuviera una temperatura de alimentación al reactor de $280{ }^{\circ} \mathrm{C}$ y una emisión de VOCs de 1 ppm. En lo que respecta al diámetro de los equipos, se estableció una relación entre el diámetro del reactor y del regenerador para que la pérdida de carga en cada equipo, cuando se opera con el contenido de VOCs nominal, sea aproximadamente igual. Con dicha relación, se ajustó el diámetro del regenerador para que la pérdida de carga total en la misma condición sea de 50 mbar. Por último, se eligió el tiempo de ciclo para la condición de operación nominal para que la temperatura máxima en el reactor sea igual a $400{ }^{\circ} \mathrm{C}$.

A partir de los criterios de diseño expuestos, se obtuvieron las dimensiones de ambos equipos para cada una de las configuraciones de celda estudiadas. Considerando los resultados obtenidos se sugirió el diseño con la configuración $2(400 / 7.5)$ para este esquema.

\subsubsection{Comparación entre los esquemas estudiados}

Finalmente, en el presente capítulo se compararon los diferentes esquemas estudiados teniendo en cuenta los diseños sugeridos en cada caso (resumidos en la Tabla 9.1). Debido a que el catalizador considerado no se ha desarrollado a nivel comercial, no se dispone de datos de costos del mismo (consecuentemente, no sería posible realizar un análisis económico cuantitativo). Por esta razón, no se contempló como alternativa de comparación una evaluación económica. En su lugar, se analizaron diferentes aspectos, señalando su posible influencia en el costo final cuando correspondiera.

En términos del espacio ocupado en planta, el esquema de inversión de flujo rotativo resultó el más favorecido, seguido del esquema regenerador-reactor. No obstante, en ningún caso se consideró el espacio adicional que pudiera ne- 
cesitarse para cualquiera de los elementos adicionales (e.g., cañerías, sopladores, etc.). Lógicamente, cuanto mayor es el volumen requerido, mayor es la masa total de material involucrado. No obstante, es conveniente discriminar la masa de material inerte y catalítico, considerando que el costo del segundo será presumiblemente mayor. De esta manera, si se considera que la cantidad de material inerte y catalítico a utilizar podría ser determinante en el costo de los esquemas, el de inversión de flujo convencional queda situado como el más desfavorable. Por otro lado, la comparación entre el esquema de inversión de flujo rotativo y el regenerador-reactor no resulta inmediata debido a la diferencia de costo esperable entre el material inerte y el catalítico. Si la relación entre el costo específico del material catalítico frente al inerte resultase superior 22, el esquema regenerador-reactor quedaría situado como el más conveniente con este criterio. En adición, también debe considerarse que la configuración de la celda puede tener un impacto sobre el costo de manufactura de los equipos, lo que nuevamente favorecería al esquema regenerador-reactor.

Por último, debe considerarse también la diferencia de complejidad de cada esquema, lo que posiblemente pueda tener algún impacto en el costo total. En este aspecto, el esquema más sencillo es el de inversión de flujo convencional, mientras que el esquema de inversión de flujo rotativo es el que presenta la mayor complejidad. Por otro lado, el esquema regenerador-reactor posee una complejidad intermedia entre los esquemas de inversión de flujo analizados, pero requiere de un soplador adicional (aunque presumiblemente no posea un peso apreciable en el costo total debido al reducido caudal que debería manejar).

A partir del análisis de cada uno de estos factores, se concluyó que el esquema de inversión de flujo convencional no resulta conveniente frente al esquema de inversión de flujo rotativo o al regenerador-reactor (será probablemente el más costoso, sin presentar alguna ventaja concluyente respecto de las dos alternativas restantes). Por otra parte, de acuerdo con los elementos considerados, tanto el esquema de inversión de flujo rotativo como el regenerador-reactor resultan igualmente aptos para el tratamiento de la corriente de aire contaminada con acetato de etilo y etanol. 


\subsection{Recomendaciones para futuros trabajos}

Durante el desarrollo del presente estudio se han identificado posibles variantes a los esquemas propuestos, así como algunos aspectos que pueden ser importantes para ampliar el conocimiento sobre los sistemas autotérmicos con intercambio de calor regenerativo y su operación. En base los mismos, surgen las siguientes alternativas para futuros trabajos:

* Como fuera discutido en el Capítulo 3, para temperaturas inferiores a los $500^{\circ} \mathrm{C}$, los regeneradores comerciales poseen típicamente una matriz de aluminio debido a su menor costo (si bien existen alternativas comerciales con matrices cerámicas, las mismas se sugieren para procesos donde la temperatura supera $\operatorname{los} 800^{\circ} \mathrm{C}$ ). A pesar de ello, se optó por considerar un material cerámico para el regenerador a fin de establecer un punto de comparación equivalente entre los esquemas. Sería interesante evaluar el impacto del uso de una matriz de aluminio para el regenerador, tanto en los requerimientos de material y dimensiones resultantes, como en la operación del sistema. Cabe resaltar que en el caso de considerar una matriz de aluminio, se deberian evaluar los posibles efectos de la conductividad del material (tanto en el sentido axial, incorporados en forma aproximada en el modelo utilizado en el presente estudio, como en el sentido angular y radial).

* En el diseño de los equipos de inversión de flujo se fijó la fracción de las regiones inertes en $40 \%$ y $50 \%$ para el esquema convencional y el rotativo, respectivamente. No obstante, se podria liberar dicha restricción y ajustar el valor de la misma de forma tal de maximizar alguna función objetivo (por ejemplo, asociada a la masa total de inerte y/o catalizador o, si se dispusiera de la relación de precios de cada uno, del costo total de materiales). Para el esquema regenerador-reactor se podría establecer una condición análoga con la temperatura de alimentación al reactor.

* En el desarrollo de los criterios, se limitó el valor de temperatura máxima del catalizador a $400{ }^{\circ} \mathrm{C}$, considerando que el mismo representa un valor suficientemente conservativo para evitar la temprana desactivación del mismo (y disponiendo aún de un cierto margen). Teniendo presente que 
dicho valor resulta importante para determinar el tiempo de ciclo para la condición de operación nominal, sería importante disponer de estudios sobre la estabilidad térmica del catalizador para ajustar la temperatura máxima a valores específicos del catalizador empleado.

Si se tiene en cuenta el elevado nivel térmico que se alcanza con cualquiera de los esquemas estudiados, pareciera razonable intentar recuperar parte de la energía liberada por la reacción (por ejemplo, generando vapor). A tal efecto, en base al esquema de inversión de flujo convencional, se han propuesto varias alternativas en bibliografia (ver, por ejemplo, Matros y Bunimovich, 1996), siendo las dos más estudiadas:

- dividir los equipos de inversión de flujo en dos secciones, intercalando un equipo de intercambio en el centro,

- extraer una fracción de la corriente de gas en la zona central del lecho para su pasaje por un equipo de intercambio y emisión a la atmósfera.

De acuerdo con Matros y Bunimovich (1996), ambas alternativas son técnicamente posibles. Si se asume que la industria de impresión de donde proviene la corriente de aire a tratar pudiera tener algún requerimiento de vapor (o pudiera venderse a empresas aledañas), la implementación de estas propuestas (y de alternativas equivalentes para el esquema de inversión de flujo rotativo y el regenerador-reactor) podría ser atractiva.

Durante el desarrollo del presente estudio surgió otro posible esquema, similar al de inversión de flujo rotativo y cuya representación se muestra en la Figura 9.1. La diferencia es que en esta propuesta la corriente a tratar no se dividiría en dos subcorrientes que se alimenten en sentidos opuestos (comparar con la Figura 3.1). En su lugar, se alimentaría la totalidad de la corriente por una región del equipo y se retornaría por la otra (Figura 9.1). De ser posible la operación autotérmica en el esquema propuesto, el retorno mencionado proporcionaria una diferencia desde el punto de vista del comportamiento del sistema: la temperatura en el punto de retorno una vez alcanzado el css sería constante en el tiempo, lo cual podría derivar en algunos beneficios. Por un lado, como conse- 
cuencia de la temperatura constante en el punto de retorno, el sistema podría tender a uniformizar la temperatura en la región catalítica, disminuyendo el valor de la temperatura máxima en el lecho. No obstante, esto presumiblemente traería aparejado una disminución en el tiempo de ciclo crítico. Por otro lado, si se quisiera extraer parte de la energía liberada por la reacción, el valor constante de la temperatura en el punto de retorno simplificaría el diseño y operación del equipo de intercambio utilizado. Por estas razones, sería interesante el análisis del esquema presentado en la Figura 9.1.

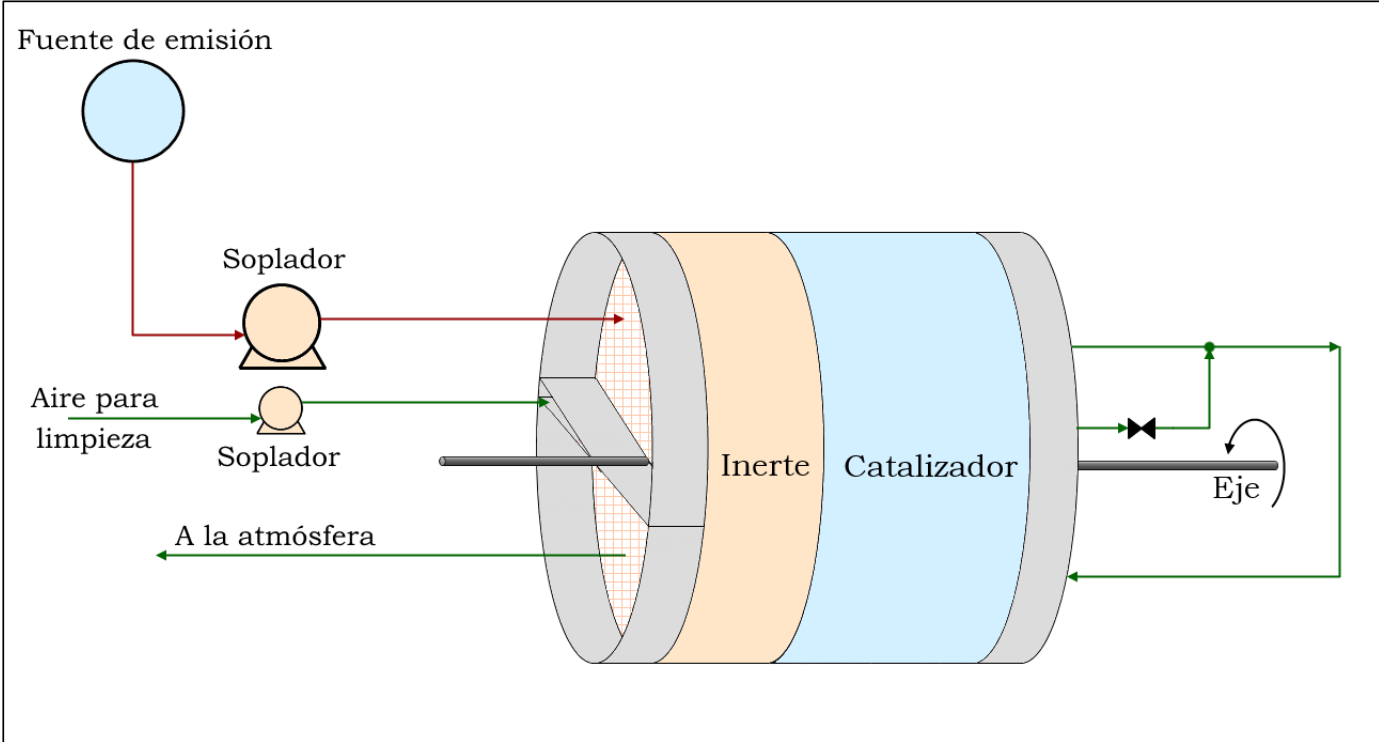

Figura 9.1: esquema de inversión de flujo rotativo con retorno. 
COMBUSTIÓN CATALÍTICA DE VOCS

UTILIZANDO SISTEMAS AUTOTÉRMICOS CON

INTERCAMBIO DE CALOR REGENERATIVO

\section{- Nomenclatura}



$\left(a / a_{0}\right)_{c r i t} \quad$ valor crítico de la actividad catalítica respecto de la actividad del catalizador fresco.

$A$ área total de intercambio en el intercambiador de calor hipotético en contra-corriente.

$\underline{\mathbf{A}}$ matriz que define el sistema lineal para la determinación del vector $\gamma^{(p)}$.

$a / a_{0} \quad$ actividad del catalizador en un momento dado respecto de la actividad del catalizador fresco.

$a_{v} \quad$ área total de transferencia por unidad de volumen total de lecho.

atol $_{T} \quad$ tolerancia absoluta para los perfiles de temperatura, utilizada en la búsqueda del css $\left(210^{-3} \mathrm{~K}\right)$.

atol $_{y} \quad$ tolerancia absoluta para la emisión global, utilizada en la búsqueda del css (5 $\left.10^{-3} \mathrm{ppm}\right)$.

$b_{-q} \quad$ coeficiente de combinación asociado al punto $z_{k-q}$, para la estimación de las derivadas en la variable axial mediante diferencias finitas atrasadas. coeficiente de seguridad utilizado en la determinación del caudal de limpieza.

$c_{p} \quad$ calor específico a presión constante.

$\bar{c}_{p G} \quad$ calor específico medio a presión constante en el gas

$C_{T} \quad$ concentración molar total en la fase gaseosa.

$d_{h} \quad$ diámetro hidráulico del canal $\left(4 \varepsilon_{L} / a_{v}\right)$.

$D_{j} \quad$ coeficiente de difusión efectivo del componente " $j$ ” en la matriz monolítica.

$D_{K, j} \quad$ coeficiente de difusión de Knudsen del componente "j" en la matriz monolítica.

$D_{m, j} \quad$ coeficiente de difusión molecular del componente " $j$ " en aire.

$d_{\text {poro }} \quad$ diámetro medio de poro de la matriz monolítica.

$D_{R} \quad$ diámetro del reactor. 
$D_{\text {Reg }} \quad$ diámetro del regenerador.

$E_{i} \quad$ energía de activación de la $i$-ésima reacción.

$E_{T} \quad$ error relativo en la predicción de la temperatura máxima para los diferentes esquemas de diferencias finitas atrasadas.

$E_{y} \quad$ error relativo en la predicción de la emisión global de VOCs para los diferentes esquemas de diferencias finitas atrasadas.

$(f \mathrm{Re}) \quad$ producto del factor de fricción de Fanning y el número de Reynolds.

$f_{E} \quad$ fracción de la sección total correspondiente a la etapa de enfriamiento del solido (regenerador).

$f_{L} \quad$ fracción de la sección total destinada a la limpieza de los canales (esquema de inversión de flujo rotativo y regenerador).

$f_{I} \quad$ fracción de la longitud de las regiones inertes frente a la longitud total (esquemas de inversión de flujo).

G velocidad másica (referida a la sección transversal total).

$\mathbf{g}(\mathbf{X}) \quad$ función hipotética que devuelve los perfiles al final de un ciclo dado, a partir de los perfiles iniciales dados por el vector $\mathbf{X}$.

$\mathbf{G}(\mathbf{X}) \quad$ función cuya raíz define el estado estacionario cíclico (css).

$G_{m, L} \quad$ caudal másico utilizado para la limpieza de los canales.

$G_{m, L, \min } \quad$ mínimo caudal másico que debe utilizarse para la limpieza de los canales.

$G_{m, t o t} \quad$ caudal másico total de la corriente a tratar.

h coeficiente de transferencia de energía.

$H_{C} \quad$ factor de calentamiento del sólido $\left(a_{v}^{2} /\left[\left(1-\varepsilon_{l}\right) \varepsilon_{L}\right]\right)$.

$h_{\text {mod }} \quad$ coeficiente de transferencia de energía modificado (incluye el efecto de la conducción térmica axial, Ec. 3.10).

In término de inhibición común de las expresiones cinéticas $\left(1+K_{1} C_{1}+K_{3} C_{3}\right)$. 
$k_{i}$

$k_{i, r e f}$

$K_{j}$

$k_{m, j}$

$K_{V}$

$L$

$L_{C}$

$L_{e q}$

$L_{M}$

$M$

$M_{\text {aire }}$

$M_{j}$

$m_{l}$

MIF

$n$

$N$

$N_{C}$

$N_{V}$ constante específica de la velocidad de la i-ésima reacción.

constante específica de la velocidad de la i-ésima reacción, evaluada a la temperatura de referencia $T_{\text {ref,cin }}$.

constante de adsorción del componente " $j$ ".

coeficiente de transferencia de materia para el componente " $j$ ".

factor de transferencia de materia o energía por unidad de volumen total $\left(a_{v}^{2} / \varepsilon_{L}\right)$.

longitud característica de la capa catalítica (relación entre el volumen de depósito y el área de transferencia de masa).

longitud de una región del equipo.

longitud de celda del monolito.

longitud total del equipo (esquemas de inversión de flujo).

longitud de un módulo de monolito.

número de iteraciones previas consideradas en el algoritmo de aceleración de la convergencia.

peso molecular del aire.

peso molecular del componente " $j$ ".

exponente que relaciona la concentración adimensional del componente " $j$ " modificado con los restantes " $l$ " componentes (Ec. 4.8).

factor de resistencia al aplastamiento en dirección diagonal.

orden considerado para el esquema de diferencias finitas atrasadas.

número de intervalos considerados para la discretización de la variable axial.

número de celdas por unidad de superficie transversal (canales $/ \mathrm{m}^{2}$ ).

número total de elementos del vector $\mathbf{x}$. 


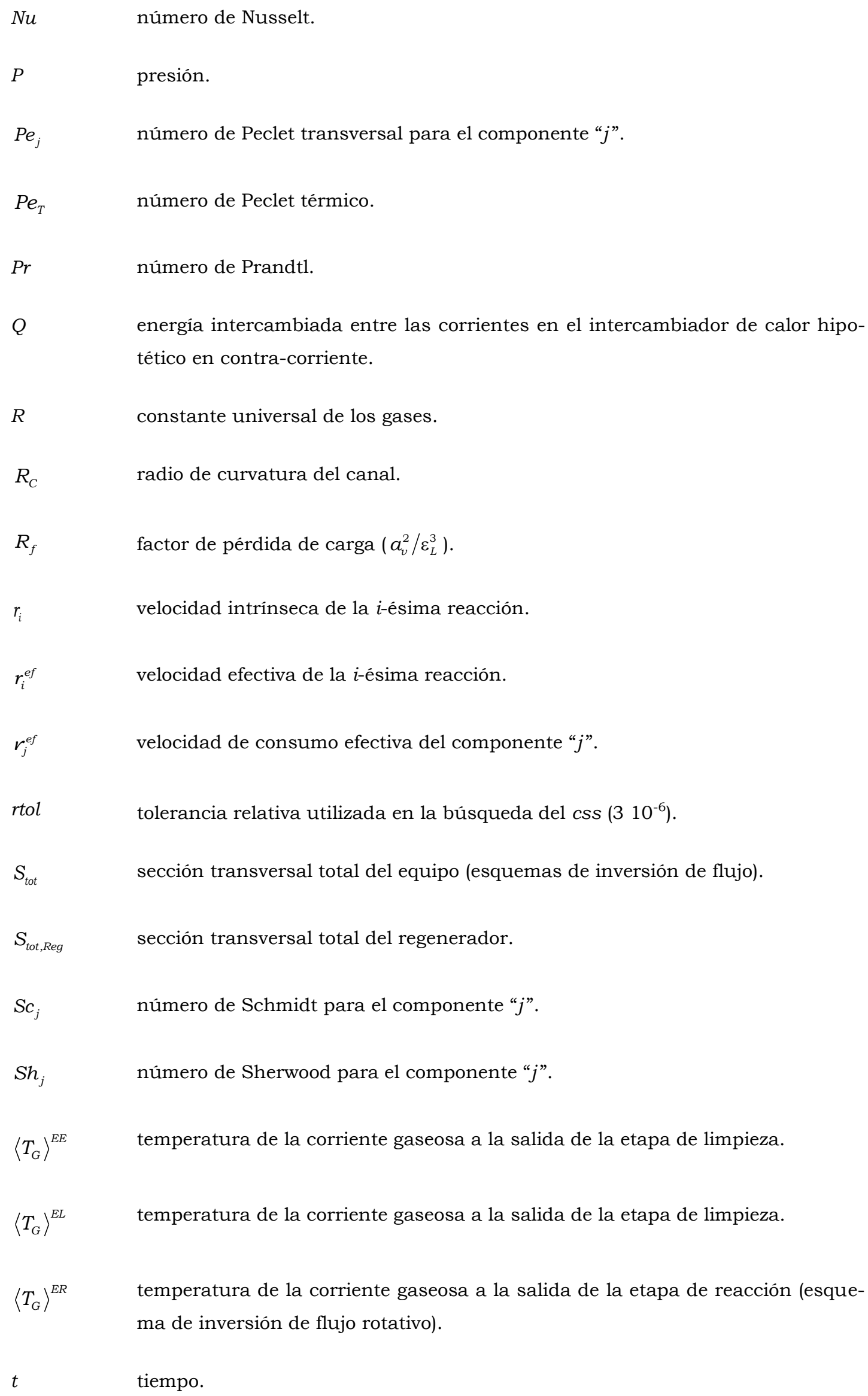


temperatura.

tiempo de cálculo requerido para la simulación hasta alcanzar el css.

tiempo total de un ciclo.

tiempo total de un ciclo para el cual la emisión de VOCs es de 9 ppm.

tiempo de duración de la etapa de calentamiento del sólido (regenerador).

tiempo de duración de la etapa de enfriamiento del sólido (regenerador).

tiempo de duración de la etapa de limpieza.

tiempo de duración de la etapa de reacción (esquema de inversión de flujo rotativo).

temperatura de ignición de los VOCs.

temperatura de referencia para el cálculo del calor específico medio a presión constante del gas.

temperatura de referencia para el cálculo de los coeficientes cinéticos.

temperatura máxima en el sólido (temporal y espacial).

valor de la temperatura máxima en el sólido considerado como "exacto", de acuerdo a los datos obtenidos con el esquema $\mathrm{DFA}_{5}$ y 500 intervalos.

coeficiente global de transferencia de energía en el intercambiador de calor hipotético en contra-corriente.

velocidad del frente de onda.

volumen necesario para el desplazamiento del frente de onda.

mínimo volumen para el precalentamiento de la corriente gaseosa hasta alcanzar la temperatura de ignición de los VOCs en los esquemas de inversión de flujo.

volumen total del reactor. 
$V_{R}^{*} \quad$ volumen mínimo de la región catalítica, suponiendo que la velocidad de reacción está controlada por la velocidad de transferencia de materia entre la corriente gaseosa y la superficie del sólido.

$V_{\text {Reg }} \quad$ volumen total del regenerador.

$w_{0}^{2} \quad$ factor interviniente en la matriz $\underline{\mathbf{A}}$, para prevenir el caso en que su determinante pudiera ser nulo.

$W_{C} \quad$ masa total de material catalitico.

$W_{I} \quad$ masa total de material inerte.

$x \quad$ conversión de acetato de etilo (Ecs. A6.1.8 a A6.1.12).

$\mathbf{x} \quad$ vector que define los perfiles iniciales en cada esquema.

$\left\langle y_{j, G}\right\rangle \quad$ fracción molar del componente " $j$ " a la salida.

$\left\langle y_{j, G}\right\rangle^{E C} \quad$ fracción molar del componente " $j$ " a la salida de la etapa de calentamiento del sólido (esquema regenerador-reactor).

$\left\langle y_{j, G}\right\rangle^{E E} \quad$ fracción molar del componente " $j$ ” a la salida de la etapa de enfriamiento del sólido (esquema regenerador-reactor).

$\left\langle y_{j, G}\right\rangle^{E L} \quad$ fracción molar del componente " $j$ " a la salida de la etapa de limpieza.

$\left\langle y_{j, G}\right\rangle^{E R} \quad$ fracción molar del componente "j" a la salida de la etapa de reacción (esquema de inversión de flujo rotativo).

$\left\langle y_{\text {VOCS }}\right\rangle \quad$ fracción molar total de VOCs a la salida.

$\left\langle y_{\text {VOCs }}\right\rangle^{r e f} \quad$ valor de referencia para la emisión de VOCs (9 ppm), utilizado en la definición de $E_{y}$.

$\left\langle y_{V O C S}\right\rangle^{\infty} \quad$ valor de la fracción molar total de VOCs a la salida considerado como "exacto", de acuerdo a los datos obtenidos con el esquema $\mathrm{DFA}_{5}$ y 500 intervalos.

$y_{j} \quad$ fracción molar del componente “ $j$ ”.

$Y_{j} \quad$ concentración del componente “j” modificado, relativa a la concentración en la superficie externa del catalizador (Ecs. 4.3 a 4.9). 
$Z^{\#}$

posición axial en el canal.

posición axial en el canal para el cual la conversión total de VOCs, evaluada a $t=t_{E R}$, corresponde al 99\% (esquema de inversión de flujo rotativo).

\section{Letras griegas}

$\alpha$

$\beta$

$\beta_{0}, \beta_{1}, \beta_{\infty}$

$\delta_{d e p}$

$\delta_{h m}$

$\delta_{j}$

$\delta_{T}$

$\underline{\Delta \mathbf{G}}$

$\Delta H_{i}$

$\Delta H_{j}^{\#}$

$|\Delta P|$

$\Delta T^{R}$

$\Delta T_{a d}$

$\Delta T_{m l}$ matriz de coeficientes estequiométricos.

coeficiente de combinación utilizado en la aproximación para las velocidades de consumo de cada componente (Ecs. 4.3 a 4.9).

constantes dependientes de la forma del canal, utilizadas en la función $\delta_{j}$ en el cálculo de los coeficientes de transferencia de energía y materia.

espesor del depósito catalítico.

función delta de Kronecker.

función del número de Schmidt del componente " $j$ ” y de la geometría del canal, utilizada en el cálculo de los coeficientes de transferencia de energía y materia.

espesor total del tabique.

matriz que registra la diferencia de la salida de la función $\mathbf{G}(\mathbf{X})$ entre las $M$ iteraciones sucesivas.

entalpía de la $i$-ésima reacción.

entalpía de combustión del componente " $j$ ”.

valor absoluto de la pérdida de carga.

salto térmico en el reactor.

máximo salto térmico adiabático en la corriente.

valor medio logarítmico del salto térmico en el intercambiador de calor hipotético en contra-corriente. 
$\underline{\Delta x}$

$\Delta z$

$\varepsilon_{C}$

$\varepsilon_{L}$

$\varepsilon_{L}^{0}$

$\eta_{j}^{*}$

$\gamma$

$\gamma^{(p)}$

$\lambda$

$\mu_{G}$

$\omega$

$\phi_{j}^{*}$

$\psi$

$\left(\rho c_{p}\right)_{G}$

$\left(\rho c_{p}\right)_{S}$

$\rho$

$\sigma^{(p)}$

$\tau_{C}$

$\mathrm{v}_{\text {aire }}$

$v_{j}$ matriz que registra la diferencia de los componentes del vector de perfiles iniciales, $\mathbf{X}$, entre las $M$ iteraciones sucesivas.

intervalo resultante de la discretización de la variable axial.

porosidad del material catalítico.

porosidad del lecho.

porosidad del lecho catalítico, previo a la deposición del catalizador.

factor de efectividad asociado a la reacción de consumo de los componentes modificados (Ecs. 4.3 a 4.9 ).

relación entre la capacidad calorifica del gas en las condiciones de alimentación y la capacidad calorífica total por unidad de volumen de lecho.

vector de combinación utilizado en el proceso de aceleración de la convergencia, asociado a la p-ésima iteración (definido mediante la Ec. 4.22).

conductividad térmica (en sentido axial en el caso de la fase sólida).

viscosidad de la corriente gaseosa.

velocidad de rotación.

Módulo de Thiele para el componente "j" modificado (Ecs. 4.3 a 4.9).

capacidad del intercambiador hipotético en contra-corriente.

capacidad calorífica por unidad de volumen de gas.

capacidad calorífica por unidad de volumen de sólido.

densidad.

parámetro de mezcla asociado a la p-ésima iteración.

tortuosidad del material catalítico.

volumen de difusión del aire.

volumen de difusión del componente “j”. 


\section{Subindices}

C propiedad o variable asociada a: catalizador, región catalítica o reactor, según corresponda. propiedad o variable asociada a la cordierita.

G propiedad o variable asociada a la fase gaseosa. $i$-ésima reacción. propiedad o variable asociada a la región inerte de los equipos o al regenerador. valores asintóticos de los parámetros de transferencia de materia y energía. variable asociada al $k$-ésimo punto considerado en la discretización de la variable axial. lido.

\section{Superindices}

$\begin{array}{ll}* & \text { variables asociadas a los componentes modificados (Ecs. } 4.3 \text { a 4.9). } \\ & \text { dirección de la corriente (izquierda a derecha) en los esquemas hipotéticos en } \\ & \text { contra-corriente. } \\ & \text { dirección de la corriente (derecha a izquierda) en los esquemas hipotéticos en } \\ & \text { contra-corriente. } \\ 0 & \text { condición de alimentación al sistema. } \\ 0, R & \text { condición de alimentación al reactor (esquema regenerador-reactor). } \\ \text { ini } & \text { condición inicial. } \\ n c & \text { número de ciclo. } \\ n s c & \text { número de semiciclo. } \\ & \\ & \text { p-ésima iteración. }\end{array}$


COMBUSTIÓN CATALÍTICA DE VOCS

UTILIZANDO SISTEMAS AUTOTÉRMICOS CON

INTERCAMBIO DE CALOR REGENERATIVO

\section{Referencias}



Barresi, A.A., Baldi, G., Fissore, D., Forced Unsteady-State Reactors as Efficient Devices for Integrated Processes: Case Histories and New Perspectives, Industrial \& Engineering Chemistry Research, 46 (25), 8693-8700 (2007).

Barresi, A.A., Vanni, M., Brinkmann, M., Baldi, G., Influence of the Wall Heat Losses on the Performance of a Network of Nonstationary Catalytic Burners, Chem. Eng. Technol., 22 (3), 237-239 (1999).

Barreto, G.F., Transferencia de materia en monolitos, Capitulo 3 en Reactores Estructurados, apunte de la cátedra "Ingeniería de Reactores Heterogéneos", Facultad de Ingeniería, Universidad Nacional de La Plata, Argentina (2008).

Bartholomew, C.H., Farrauto, R.J., Fundamentals of industrial catalytic processes, 2da edición, John Wiley \& Sons (2006).

Boger, T., Heibel, A., Sorensen, C.M., Monolithic Catalysts for the Chemical Industry, Industrial \& Engineering Chemistry Research, 43 (16), 4602-4611 (2004).

Boreskov, G.K, Matros, Yu.Sh., Unsteady-state performance of heterogeneous catalytic reactions, Catal. Rev., 25 (4), 551-590 (1983).

Brinkmann, M., Barresi, A.A., Vanni, M., Baldi, G., Unsteady state treatment of very lean waste gases in a network of catalytic burners, Catalysis Today, 47, 263-277 (1999).

Bunimovich, G., Sapoundjiev, H., Periodic Flow Reversal, Cap. 18 en Periodic Operation of Chemical Reactors, $1^{\text {ra }}$ edición (Eds. Silveston P.L. y Hudgins, R.R.), Elsevier (2013).

Bunimovich, G.A., Sapundzhiev, Ch., Matros, Yu.Sh., Drobishevich, V.I., Yausheva, L.V., Elenkov, D., Grozev, G., Mathematical simulation of temperature unhomogeneities in a flow reversal reactor, en Unsteady State Processes in Catalysis: Proceedings of the International Conference (Ed. Matros, Yu.Sh.), VPS BV, Utrecht, 461-470 (1990).

Burden, R.L., Faires, J.D., Numerical Analysis, 9na edición, Brooks/Cole CENGAGE Learning (2011).

Campesi, M.A., Estudio de Sistemas Combinados de Combustión Catalitica de VOCs. Tesis Doctoral, Universidad Nacional de La Plata, Argentina (2012).

Campesi A., Luzi C.D., Martinez O.M., Barreto G., Effect of Concentration by Thermal Swing Adsorption on the catalytic Incineration of VOCs, International Journal of Chemical Reactor Engineering, 10 (1), A54 (2012a).

Campesi, M.A., Mariani, N.J., Bressa, S.P., Pramparo, M.C., Barbero, B.P., Cadús, L.E, Barreto, G.F., Martinez, O.M., Kinetic study of the combustion of ethanol and ethyl acetate mixtures over a $\mathrm{MnCu}$ catalyst, Fuel Processing Technology, 103, 84-90 (2012b). 
Campesi, M.A., Mariani, N.J., Martínez, O.M., Barreto, G. F., Eliminación de COVs mediante un proceso de oxidación catalitica con recuperación térmica, Ingeniería Mecánica, Tecnología y Desarrollo, 2 No. 4, 111-119 (2007).

Chen, G., Chi, Y., Yan, J., Ni M., Effect of Periodic Variation of the Inlet Concentration on the Performance of Reverse Flow Reactors, Ind. Eng. Chem. Res., 50 (9), 5448-5458 (2011).

Cittadini, M., Vanni, M., Barresi, A.A., Baldi, G., Simplified procedure for design of catalytic combustors with periodic flow reversal, Chemical Engineering and Processing, 40 (3), 255-262 (2001).

Cottrell, F.G., Purifying gases and apparatus therefore, US Patent 121, 733, US Patent Office, Washington, DC, 1938.

de la Fuente García, D., Gutiérrez Martín, F., Optimización de sistemas de destrucción de VOCs, Ingeniería Química, 151-158 (1998).

Directiva 2010/75/UE del Parlamento Europeo y del Consejo, sobre las emisiones industriales (prevención y control integrados de la contaminación) (2010). En EUR-Lex. Recuperado el 3 de marzo de 2015 de

http://eur-lex.europa.eu/legalcontent/ES/TXT/?qid=1422968493087\&uri=CELEX:32010L0075.

Eigenberger, G., Kolios, G., Nieken, U., Thermal pattern formation and process intensification in chemical reaction engineering, Chemical Engineering Science, 62 (18-20), 4825-4841 (2007).

Eigenberger, G., Nieken, U., Catalytic cleaning of polluted air: reaction engineering problems and new solutions, International Chemical Engineering, 34 (1), 4-16 (1994).

Eigenberger, G., Nieken, U., Catalytic combustion with periodical flow reversal, Chemical Engineering Science, 43 (8), 2109-2115 (1988).

Everaert, K., Baeyens, J., Creemers, C., A comparative study of abatement techniques for volatile organic compounds (VOCs), Proc. of CHISA 2002 - 15th Int. Congress of Chemical and Process Eng., Praga, Zchec Republic (2002).

Eyert, V., A Comparative Study on Methods for Convergence Acceleration of Iterative Vector Sequences, Journal of Computational Physics, 124 (2), 271-285 (1996).

Fissore, D., Barresi, A.A., Baldi, G., Hevia, M.A.G., Ordóñez, S., Diez, F.V., Design and Testing of Small-Scale Unsteady-State Afterburners and Reactors, AIChE Journal, 51 (6), 1654-1664 (2005).

Fissore, D., Barresi, A.A., Comparison between the Reverse-Flow Reactor and a Network of Reactors for the Oxidation of Lean VOC Mixtures, Chem. Eng. Technol., 25 (4), 421-426 (2002). 
Fuller, E.N., Schettler, P.D., Giddings, J.C., A new method for prediction of binary gas-phase diffusion coefficients, Industrial and Engineering Chemistry, 58 (5), 18-27 (1966).

Ghoshal, A.K., Manjare, S.D., Selection of appropriate adsorption technique for recovery of VOCs: an analysis, Journal of Loss Prevention in the Process Industries, 15 (6), 413-421 (2002).

Gosiewski, K., Pawlaczyk, A., Catalytic or thermal reversed flow combustion of coal mine ventilation air methane: What is better choice and when?, Chemical Engineering Journal, 238, 78-85 (2014).

Gulati, S.T., Ceramic Catalyst Supports for Gasoline Fuel, Cap. 2 en Structured Catalysts and Reactors, 2nd Edition (Eds. Cybulski, A. y Moulijn, J.A.), Taylor and Francis (2006).

Haynes, T.N., Caram, H.S., The simulated moving bed chemical reactor, Chemical Engineering Science, 49 (24b), 5465-5472 (1994).

Hesselgreaves, J.E., Compact heat exchangers: Selection, design and operation, 1ra edición, Pergamon (2001).

Hevia, M.A.G., Ordóñez, S., Diez, F.V., Effect of the catalyst properties on the performance of a reverse flow reactor for methane combustion in lean mixtures, Chemical Engineering Journal, 129 (1-3), 1-10 (2007).

Hodgson A.T., Destaillats H., Sullivan D.P., Fisk. W.J., Performance of ultraviolet photocatalytic oxidation for indoor air cleaning applications, Indoor Air, 17 (4), 305-316 (2007).

Jacoby W.A., Blake D.M., Fennell J.A., Boulter J.E., Vargo L.M., George M.C., Dolberg S.K., Heterogeneous photocatalysis for control of volatile organic compounds in indoor air, J. Air \& Waste Manag., 46, 891-898 (1996).

Khan, F.I., Ghoshal, A.Kr., Removal of volatile organic compounds from polluted air, Journal of Loss Prevention in the Process Industries, 13, 527-545 (2000).

Kolaczkowski, S., Treatment of Volatile Organic Carbon. Emmisions from Stationary Sources: Catalytic Oxidation of the Gaseous Phase, Cap. 5 en Structured Catalysts and Reactors, 2nd Edition (Eds. Cybulski, A. y Moulijn, J.A.), Taylor and Francis (2006).

Kolios, G., Frauhammer, J., Eigenberger, G., Autothermal fixed-bed reactor concepts, Chemical Engineering Science, 55 (24), 5945-5967 (2000).

Leson G., Winer A.M., Biofiltration: an innovative air pollution control technology for VOC emissions, J. Air Waste Manage Assoc., 41 (8), 1045-1054 (1991).

Lewandowski, D.A., Design of Thermal Oxidation Systems for Volatile Organic Compounds, CRC Press (2000). 
Madai, A.Y., Sheintuch, M., Demonstration of Loop Reactor Operation, AIChE Journal, 54 (9), 2413-2422 (2008).

Marín, P., Ordóñez, S., Diez, F.V., Monoliths as Suitable Catalysts for Reverse-Flow Combustors: Modeling and Experimental Validation, AIChE Journal, 56 (12), 3162-3173 (2010).

Marín, P., Ordóñez, S., Diez, F.V., Simplified design methods of reverse flow catalytic combustors for the treatment of lean hydrocarbon-air mixtures, Chemical Engineering and Processing, 48 (1), 229-238 (2009).

Marín, P., Hevia, A.G., Ordóñez, S., Díez, F.V., Combustion of methane lean mixtures in reverse flow reactors: Comparison between packed and structured catalyst beds, Catalysis Today, 105 (3-4), 701-708 (2005).

Matros, Yu.Sh., Catalytic Processes under unsteady-state conditions, Studies in Surface Science and Catalysis, 43, 1-403 (1989).

Matros, Yu.Sh., Bunimovich, G.A., Reverse-Flow Operation in Fixed Bed Catalytic Reactors, Catalysis Review, 38 (1), 1-68 (1996).

Matros, Yu.Sh., Bunimovich, G.A., Strots, V.O., Mirosh, E.A., Reversed flow converter for emission control after automotive engines, Chemical Engineering Science, 54 (13-14), 2889-2898 (1999).

Matros, Yu.Sh., Bunimovich, G.A., Petterson, S.E., Meyer, S.F., Is it economically feasible to use heterogeneous catalysts for VOC control in regenerative oxidizers?, Catalysis Today, 27 (1-2), 307-313 (1996).

Matros, Yu.Sh., Noskov, A.S, Chumachenko, V.A., Progress in reverse-process application to catalytic incineration problems, Chemical Engineering and Processing, 32(2), 89-98 (1993).

Morales, M.R., Barbero, B.P., Cadús, L.E., Evaluation and characterization of Mn-Cu mixed oxide catalysts for ethanol total oxidation: Influence of copper content, Fuel, 87 (7), 1177-1186 (2008).

Moretti, E.C., Reduce VOC and HAP Emissions, Chem. Eng. Prog., 98, 30-40 (2002).

Nieken, U., Kolios, G., Eigenberger, G., Limiting Cases and Approximate Solutions for Fixed-Bed Reactors with Periodic Flow Reversal, AIChE Journal, 41 (8), 1915-1925 (1995).

Passalia C., Alfano O.M., Brandi R.J., A methodology for modeling photocatalytic reactors for indoor pollution control using previously estimated kinetic parameters, J. Hazardous Material, 211212, 357-365 (2012).

Pinjala, V., Chen, Y.C., Luss, D., Wrong-way behavior of packed-bed reactors: II. Impact of thermal dispersion, AIChE Journal, 34 (10), 1663-1672 (1988). 
Poling, B.E., Thomson, G.H., Friend, D.G., Rowley, R.L., Wilding, W.V., Physical and Chemical Data, Capítulo 2 en Perry's Chemical Engineering Handbook, 8va Edición (Eds. Perry, R.H. y Green, D.,W.), McGraw-Hill (2008).

Product Catalogue - Heat Exchanger (2013). En Kastt. Recuperado el 18 de junio de 2014 de http://www.kastt.cz/en/products\#downloads 1

Ray M.B., Photodegradation of the Volatile Organic Compounds in the Gas Phase: A Review, Developments in Chemical Engineering and Mineral Processing, 8 (5-6), 405-439 (2000).

Regenerative heat recovery with rotary heat exchangers (2008). En Klingenburg - Energy Recovery. Recuperado el 18 de junio de 2014 de

http://www.klingenburg.de/en/products/rotor-documents.html.

Regenerative Heat Exchangers For Industry and Process Technology (2003). En Klingenburg Energy Recovery. Recuperado el 18 de junio de 2014 de

http://www.klingenburg.de/en/products/htr-documents.html

Ruthven, D.M., Principles of adsorption and adsorption processes, John Wiley \& Sons (1984).

Salomons, S., Modelling the Behaviour of a Reverse-Flow Catalytic Reactor for the Combustion of Lean Methane, Tesis de Maestria, University of Alberta, Canada (2003).

Shah, R.K., Sekulic, D.P., Fundamentals of Heat Exchanger Design, John Wiley \& Sons (2003).

Shah, R.K., Thonon, B., Benforado, D.M, Opportunities for heat exchanger applications in environmental systems, Applied Thermal Engineering, 20, 631-650 (2000).

Shampine, L.F., Reichelt, M.W., The MATLAB ODE Suite, SIAM Journal on Scientific Computing, 18 (1), 1-22 (1997).

Shampine, L.F., Reichelt, M.W., Kierzenka, J.A., Soving Index-1 DAEs in MATLAB and Simulink, SIAM Review, 41 (3), 538-552 (1999).

Stankiewicz, A.I., Moulijn, J.A., Process Intensification Transforming Chemical Engineering, Chemical Engineering Progress, 96 (1), 22-33 (2000).

Technical conditions for rotary exchangers for heat recovery (s.f.). En Kastt. Recuperado el 5 de noviembre de 2014 de http://kastt.cz/en/products\#f.

Thermal Oxidizers. (s.f.). En Gulf Coast Environmental Systems. Recuperado el 10 de junio de 2014, de http://www.gcesystems.com/thermal-oxidizers.html.

Thullie, J., Burghardt, A., Simplified procedure for estimating maximum cycling time of flowreversal reactors, Chemical Engineering Science, 50 (14), 2299-2309 (1995). 
van de Beld, B., Westerterp, K.,R., Air Purification by Catalytic Oxidation in a Reactor with Periodic Flow Reversal, Chem. Eng. Technol., 17 (4), 217-226 (1994).

Vanden Bussche, K.M., Neophytides, S.N., Zolotarskii, I.A., Froment, G.F., Modelling and simulation of the reveresed flow operation of a fixed-bed reactor for methanol synthesis, Chemical Engineering Science, 48 (19), 3335-3345 (1993).

Vortmeyer, D., Schaefer, R.J., Equivalence of one- and two-phase models for heat transfer processes in packed beds: one dimensional theory, Chemical Engineering Science, 29, 485-491 (1974).

Willmott, A.J., Dynamics of regenerative heat transfer, Taylor \& Francis (2002).

Zagoruiko, A., Modeling of Reverse-Flow Reactor for VOC Incineration with Account of Reversible Adsorption: The Way to Minimize the Negative Influence of Desorption Phenomena, International Journal of Chemical Reactor Engineering, 6 (1), A110 (2008). 